\title{
Photoelectron Spectroscopy of Large Water Cluster Anions
}

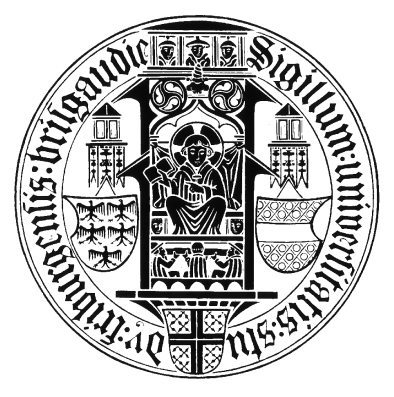

Inaugural-Dissertation

zur Erlangung des Doktorgrades

der Fakultät für Mathematik und Physik

der Albert-Ludwigs-Universität

Freiburg im Breisgau

vorgelegt von

Kiran Majer

Freiburg, Juli 2016 
Dekan: Prof. Dr. Dietmar Kröner

Leiter der Arbeit: Prof. Dr. Bernd von Issendorff Referent: Prof. Dr. Bernd von Issendorff

Koreferent: Prof. Dr. Frank Stienkemeier

Datum der mündlichen Prüfung: 23. Sept. 2016 


\section{Contents}

$\begin{array}{ll}\text { 1. Introduction } & 7\end{array}$

2. Experimental Setup 11

2.1. Cluster Source. . . . . . . . . . . . . . . . . . . . . . . . . . . . . 11

2.1.1. Oven . . . . . . . . . . . . . . . . . . . . . . . . . . . . 13

2.1.2. Source for Liquids. . . . . . . . . . . . . . . . . . . . . . . . . . . . . . . . . . 14

2.1.3. Magnetron Sputter Source . . . . . . . . . . . . . . . . . . . 16

$2.2 . \quad$ Ion Optics . . . . . . . . . . . . . . . . . . . . . . . . 16

2.3. Ion Optics Between Source and Thermalization Stage . . . . . . . . 19

2.4. Ion Trap and Thermalization Stage . . . . . . . . . . . . . . . . . 20

2.4.1. Thermalization of clusters . . . . . . . . . . . . . . 21

2.5. Mass Selection and Spectroscopy . . . . . . . . . . . . . . . . 22

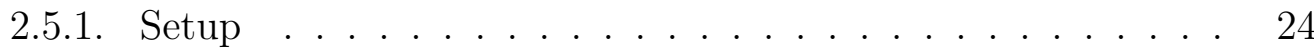

$2.5 .2 . \quad$ Mass Filter . . . . . . . . . . . . . . . . . . . . . . . . 24

2.5.3. Ion Detector . . . . . . . . . . . . . . . . . . . . . . . . . . . . . . . . 25

2.6. Photoelectron Spectroscopy . . . . . . . . . . . . . . . . . 25

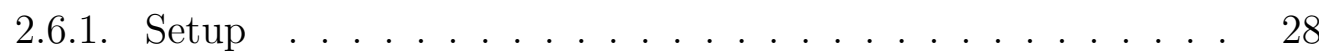

2.6.2. Influence of the cluster temperature . . . . . . . . . . . . . . . . . . 30

2.7. Vacuum System and Laser . . . . . . . . . . . . . . . . . . . 31

2.7.1. Vacuum System . . . . . . . . . . . . . . . . . . . . . 31

2.7 .2$. Laser . . . . . . . . . . . . . . . . . . . . . . . 32

2.8. Data Acquisition and Analysis . . . . . . . . . . . . . . . . . 33

2.8.1. Mass Spectra and Calibration . . . . . . . . . . . . . . 33

2.8.2. Photoelectron Spectra and Calibration . . . . . . . . . . . . 34

Discussion . . . . . . . . . . . . . . . . . . 36

2.8.3. Issues Recording Photoelectron Spectra of Water Cluster An-

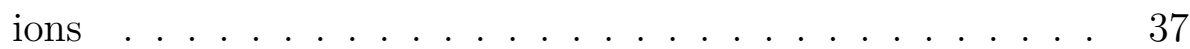

"Echo" Peaks in Early Measurements . . . . . . . . . . . . . 39

Detector artifacts . . . . . . . . . . . . . . . . . . . . 41

Decreasing Signal Intensity with Increasing Cluster Size. . . 42

3. Theoretical Background 43 
4. Water Cluster Anions 47

$4.1 . \quad$ Experimental Data . . . . . . . . . . . . . . . . . . . . . 47

$4.1 .1 . \quad$ Mass Spectra . . . . . . . . . . . . . . . . . . 47

4.1.2. $\quad$ Photoelectron Spectra . . . . . . . . . . . . . . . . . . . . . 49

4.2. Peak Analysis . . . . . . . . . . . . . . . . . . . . 50

4.2.1. Method ........................... 50

4.2.2. First Approximation: Single GL Fit . . . . . . . . . . . . 51

Sizes 15 to 27 . . . . . . . . . . . . . . 51

Sizes 28 to 33 . . . . . . . . . . . . . . . 52

Sizes 34 to 49 . . . . . . . . . . . . . . . . . . 53

Sizes 50 to 53 . . . . . . . . . . . . . . . . 54

Sizes 54 to 66 . . . . . . . . . . . . . . . . 54

Sizes 67 to 1100 . . . . . . . . . . . . . . . . . 55

4.2.3. Results of the Single GL Fit Analysis . . . . . . . . . . . . 55

$4.2 .4 . \quad$ Discussion Single GL Fit Results . . . . . . . . . . . . . . . 58

4.2.5. Refinement: Multi GL Fit . . . . . . . . . . . . . . . . 60

Two GL Fit Analysis . . . . . . . . . . . . . . . . . . 61

Discussion of the 2 GL Fit Analysis . . . . . . . . . . . . 66

3 GL Fit Analysis . . . . . . . . . . . . . . . . . . . . . . . . . . . 68

Discussion of the 3 GL Analysis . . . . . . . . . . . . . . . 72

4.2 .6$. Temperature Dependency . . . . . . . . . . . . . 74

Discussion of the Temperature Dependency. . . . . . . . . 80

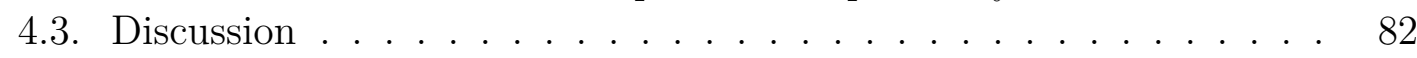

4.3.1. Comparison with external experimental data . . . . . . . . . 85

Experimental Peak Shapes . . . . . . . . . . . . . . . . . 89

The Gaussian-Lorentzian as Fit Function . . . . . . . . . . . 90

Magic Sizes . . . . . . . . . . . . . . . . . . . . 99 91

4.3.2. Comparison with theoretical data . . . . . . . . . . . . . 92

4.3.3. Dielectric Sphere Model and Permittivities . . . . . . . . . . 96

$\begin{array}{lr}\text { 5. Heavy Water Cluster } & 101\end{array}$

5.1. Experimental Data . . . . . . . . . . . . . . . . . . . . 101

$5.2 . \quad$ Peak Analysis . . . . . . . . . . . . . . . . . . . . . . . 102

5.2.1. Single GL Fit Analysis . . . . . . . . . . . . . . . . . . . 102

Impact of Deuteration on the Single Fit Results . . . . . . . 103

5.2.2. Multi GL Fit Analysis . . . . . . . . . . . . . . . . . 104

2 GL Fit Analysis . . . . . . . . . . . . . . . . . . . . . . 104

Comparison with the $\left(\mathrm{H}_{2} \mathrm{O}\right)_{\mathrm{n}}{ }^{-} 2$ GL Fit Results . . . . . . 106

Multi GL Analysis . . . . . . . . . . . . . . . 106

Comparison to the $\left(\mathrm{H}_{2} \mathrm{O}\right)_{\mathrm{n}}{ }^{-}$Multi GL Fit Results . . . . . . 108

5.2.3. Temperature Dependency . . . . . . . . . . . . . 108 
5.3. Discussion . . . . . . . . . . . . . . . . . . . . . . . . . 110

Comparison with experimental data of other groups . . . . . 112

\begin{tabular}{ll}
\hline 6. Conclusions & 115
\end{tabular}

$\begin{array}{ll}\text { 7. Summary and Outlook } & 121\end{array}$

Outlook . . . . . . . . . . . . . . . . . . . 122

\begin{tabular}{ll}
\hline A. Photoelectron Spectra & 125
\end{tabular}

A.1. Water Cluster Anions $(590 \mathrm{~nm})$. . . . . . . . . . . . . . 125

A.2. Water Cluster Anions $(248.4 \mathrm{~nm})] \ldots \ldots \ldots \ldots$

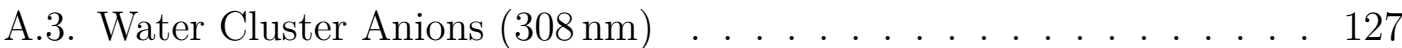

A.4. Deuterated Water Cluster Anions $(308 \mathrm{~nm})$. . . . . . . . . . . 149

B. Temperature Dependency 155

Water Cluster Anions. . . . . . . . . . . . . . . . . . 155

Deuterated Water Cluster Anions . . . . . . . . . . . . . . 171

C. CLUSIS a Python Framework for a CLUster Spectra Information System173 Principles . . . . . . . . . . . . . . . . . . . . 173

Features . . . . . . . . . . . . . . . . . . . . . 174

Future Plans . . . . . . . . . . . . . . . . . 174 



\section{Introduction}

Clusters are conglomerations of atoms or molecules. Cluster sizes range from the dimer up to nano particles or droplets consisting of several thousand atoms or molecules. The key feature of cluster science are size selected experiments, i.e. the investigation of physical properties of the cluster in dependency of its size. Experiments on size selected clusters allow to study the evolution of the properties of (bulk) matter from its building blocks, the atoms or molecules. The form matter assumes in this intermediate size range is far from being easily predictable and so, now and then clusters reveal astonishing physical and chemical properties [31].

By adding more and more atoms or molecules to the cluster, all cluster properties must eventually evolve into the properties of the bulk. The cluster can therefore be viewed as a finite-sized analogue or precursor of the bulk. By investigating the size dependent development of a property and extrapolating it to ever-increasing cluster sizes, a new possibility opens to determine an estimate of the bulk value of that property.

Water cluster anions $\left(\mathrm{H}_{2} \mathrm{O}\right)_{\mathrm{n}}{ }^{-}$, consisting of $n$ water molecules plus the additional electron, are considered as such a finite-sized analogue of excess electrons solvated in water. That liquids are able to solvate "free" electrons is a long known fact in chemistry. The deep colors of solutions of alkali metals in ammonia was early attributed to a new ionic species [80], which was later identified as the electron [49]. The solvated electron constitutes therefore the simplest possible anion. Absorption measurements by Hart and Boag confirmed the existence of the solvated electron in aqueous solutions including water [30]. Due to the high relevancy of water as a universal solvent in biology and chemistry, its solvated electron is often referred to as hydrated electron ${ }^{1}$. It is a fundamental transient species in the radiation chemistry of water and has therefore in conjunction with the widely spread occurrence of water implications on a large number of subjects in radiation chemistry and biology.

The evidence for the existence of the hydrated electron entailed a bunch of questions about its nature, some of which still eludes a final answer. Among the most fundamental ones are the grade of solvation, i.e. the electron affinity of liquid water and how the hydrated electron is embedded in the solvent.

With the first observation of water cluster anions [2] a new approach to hydrated electron investigations was opened through size-dependent measurements and extrapolation. Since then a large number of experimental and theoretical studies has

\footnotetext{
${ }^{1}$ Or sometimes as aqueous electron.
} 
been undertaken. One focus lay on the measurement of the vertical detachment energy $\mathrm{VDE}=E_{\text {anion }}-E_{\text {neutral }}$ of the hydrated electron. A series of photoelectron spectroscopy measurements of water cluster anions revealed an approximately linear dependency of the VDE with the cluster radius for medium sized water cluster anions [16], which could be explained by a model placing the excess electron in the interior of a dielectric sphere[5]. For the very small cluster sizes, two isomers, distinguishable by their different VDEs, were identified experimentally [16, 43]. The possibility of two isomers in experimental cluster beams, one with a surface and one with a interior localized electron, was already predicted by theoretical models [5], which initiated the debate on the assignment of the experimental findings to the respective isomers. The higher binding energy isomer was attributed to the interior state by experimentalists on account of its linearly developing VDE and its presence in the largest measured cluster sizes. A comparison with calculated VDEs[5], however, associated it rather with the surface state. Makov and Nitzan demonstrated later that a linear size dependency of the VDEs derived from the dielectric sphere model is not necessarily confined to interior localized isomers only, but also applies to surface localized ones[57].

In subsequent photoelectron spectroscopy measurements new isomer types with lower VDEs were discovered in very cold water and deuterated water clusters[17, 77]. However, since no higher binding energy isomers were found[41], the original assignment of the tightest bound isomer to the interior state was kept. The first series of water cluster photoelectron measurements performed with our setup revealed evidence of two high binding energy isomers instead of the formerly reported highest binding energy isomer [54]. This was a first hint that the temperature conditions of the experiment may also influence the shape of the main peak of the photoelectron spectra. Temperature dependent measurements carried out using our setup, confirmed the temperature dependency of the spectra for some cluster sizes. Other groups also reported a small temperature effect on the VDEs [82].

By now, several groups succeeded in measuring the VDEs of of the solvated electron in liquid water-jets, which is currently the method that comes closest to measuring the VDE of the bulk hydrated electron directly. The VDEs range from $3.27 \mathrm{eV}$ to $3.6 \mathrm{eV}[35,53,66,68,61]$, indicating that this method has its own fair share of error sources.

The elusive nature of the hydrated electron and its localization inspired many theoretical groups to develop new theoretical approaches more sophisticated models of water clusters. More types of electron localization were predicted, like diffuse surface structures[6], surface cavity structures[36] or interior non-cavity localizations [51, 72]. The VDEs gained from different models show, however, an even larger spread than the experimental data from different setups. The assignment of the VDEs to structures with different electron localizations remained therefore ambiguous.

The current state of research places the VDE of the hydrated electron of the 
bulk in a range of $3.27 \mathrm{eV}$ to $4.0 \mathrm{eV}$, either by direct measurements (water-jets) or extrapolations. The experimental results from cluster studies show some discrepancies in the VDEs of larger sizes, but concur in their findings that the isomer with the highest binding energy grows into the bulk hydrated electron and therefore represents an interior state. Some theoretical calculations support this assignment, whereas others still challenge it. All told, this leaves us with a bunch of questions: How does the VDE continue to develop for larger cluster sizes? How many isomers contribute to the experimental spectrum? Which are the dominant isomers? Exist isomers with even higher VDEs than the current extrapolations suggest? How does the cluster temperature influences the measured VDEs? And how valid is the comparison of extrapolated VDEs from cold, solid clusters with liquid water-jet measurements?

This work addresses these question. We have increased the experimental cluster size range by a factor 5 to 10 to very large water clusters consisting of more than 1000 water molecules. In the light of the new results the small and medium sized clusters are reevaluated. A detailed line shape analysis allows a better understanding of the discrepancies in experimental findings. Measurements of deuterated water cluster anions $\left(\mathrm{D}_{2} \mathrm{O}\right)_{\mathrm{n}}{ }^{-}$complement the results of the non-deuterated cluster experiments and give further insides into the origin of the experimental line shapes. Temperature dependent measurements try to shed some light on the impact of the cluster temperature on experimental spectra. 



\section{Experimental Setup}

An experimental study of clusters in the gas phase involves in general the following steps: Cluster production, preparation and selection of a certain type of clusters, and the actual investigation by spectroscopic means. Gas phase clusters, in contrast to deposited or immersed clusters, have no environment to interact with. They can only exist in a free cluster beam. Consequently all stages of the experiment must be performed under ultra high vacuum (UHV) conditions, and therefore are housed in a series of interconnecting vacuum chambers. Like the majority of experiments on free clusters, our setup is constructed for ionized clusters. This is due to the fact that charged particles are much easier to control and manipulate than their neutral counterparts. Ion optics, electrodes producing electrical fields in which ions undergo certain forces, are the building blocks for such experiments.

An overview of the essential parts of the experimental setup is displayed in fig. 2.1; Cluster production and ionization take place in the aggregation tube. The cluster ions are then guided into the ion trap, where they are thermalized to adopt a defined temperature. The accelerator, the reflectors and the ion detector form together the time-of-flight (TOF) mass spectrometer for cluster mass analysis. It allows, in conjunction with the mass gate, to prepare an ensemble of clusters of a single size for spectroscopic investigation. Currently two types of spectroscopy can be realized with our setup. Photoelectron spectroscopy measures the kinetic energy of electrons detached from clusters through interaction with laser photons. Photofragmentation spectroscopy uses the mass spectrometer to analyze the abundance of cluster fragments after laser irradiation. It is an experimental technique to determine caloric properties like the caloric curve, melting point and heat capacity of clusters 63 .

\subsection{Cluster Source}

Clusters are formed by condensation: Atoms or molecules of the cluster material are brought into the gas phase and cooled down till they form a supersaturated vapor in which aggregation becomes possible. By now various types of cluster sources are in use. They differ mainly in how the material is vaporized and how the vapor is cooled subsequently. Whereas various methods for material evaporation exist, the cooling is realized mainly by two processes: Heat exchange by collisions with cold rare-gas atoms (gas aggregation) and supersonic expansion. 


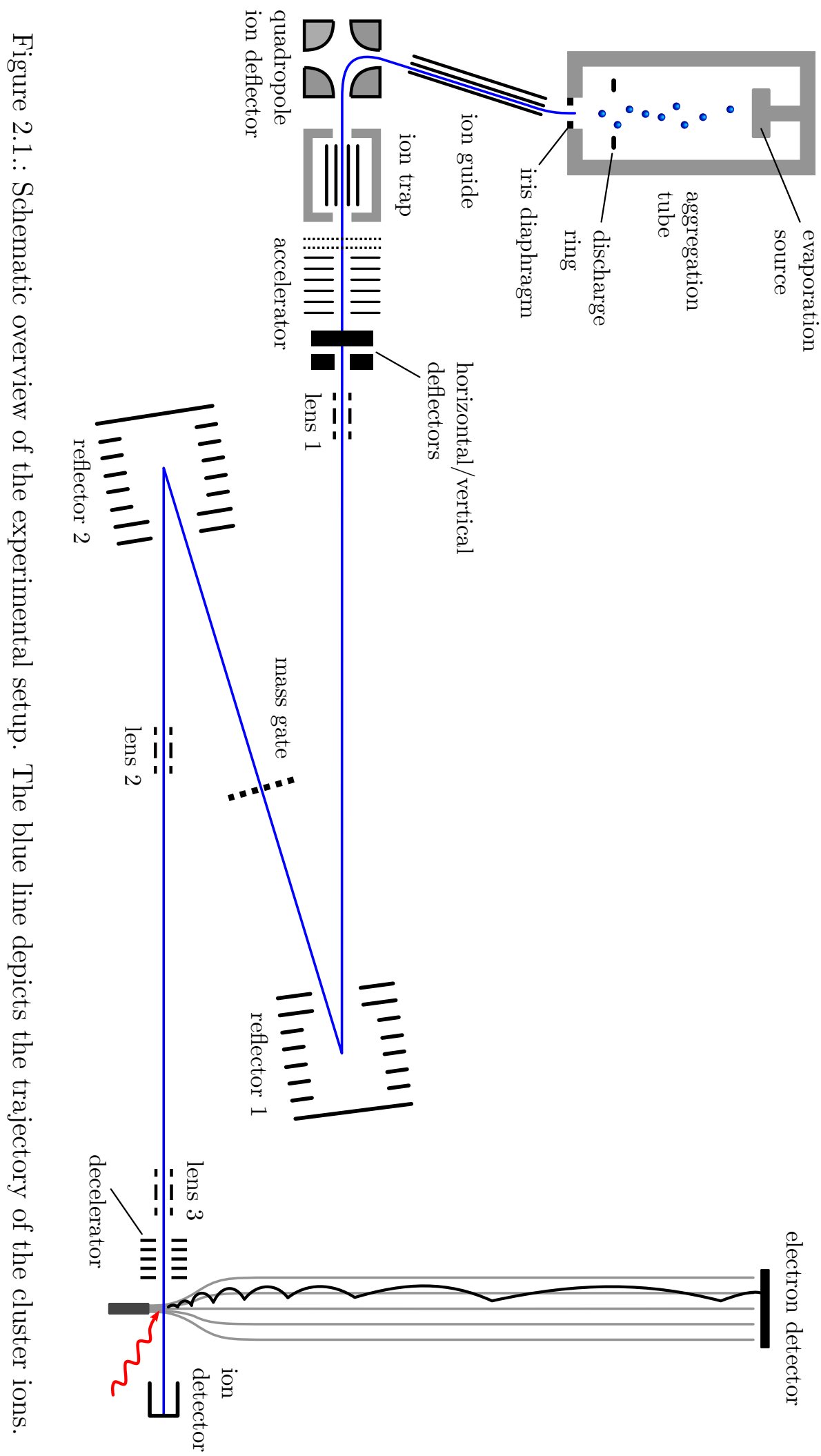


In our experiment clusters are produced by a gas aggregation source. In it the condensation process proceeds as follows: The cluster material is evaporated into a cold rare-gas atmosphere. The monomers and dimers loose more and more kinetic energy by repeatedly colliding with gas atoms. As soon as the kinetic energy becomes lower than the binding energy, condensation nuclei can be formed by means of three-body collisions:

$$
M+M+A r \rightarrow M_{2}+A r
$$

$M$ indicates here an atom or molecule of the cluster material and $A r$ a rare gas atom. Three-body collisions are necessary for energy conservation. Clusters consisting of few atoms or molecules can be formed according to this scheme. Larger clusters grow by taking up further atoms. The energy released in this process can be distributed among the larger number of inner degrees of freedom without destroying the cluster. Consequently the cluster heats up. The excessive heat can be dissipated by super-elastic collisions with the cold buffer gas.

Our source consists of a double-walled, liquid nitrogen cooled aggregation tube and various evaporation devices, which can be mounted into it. The aggregation tube possesses gas inlets on one side and a variable aperture (iris diaphragm) on the other side. A cold rare gas atmosphere of certain composition (mostly He and/or Ar) and pressure can be produced by adjusting the amount of gas flowing into the tube and the aperture width of the outlet. Composition, pressure and flow velocity of the buffer gas strongly influence the cluster growth. They are important parameters to control the size and abundance distribution of the produced clusters.

The ionization of the clusters takes place either very early in the growth process and/or at the end, depending on which vaporization source is used. The choice of the vaporization source in turn depends on the matter of which the clusters should be formed. The different sources are described in greater detail in the following subsections.

\subsubsection{Oven}

Materials with low melting points $\left(<200^{\circ} \mathrm{C}\right)$ can be vaporized by heating them well above their melting point. At a certain, material dependent temperature the vapor density is high enough for cluster formation. The usage of ovens is limited eventually by the cooling capacity of the aggregation tube. At an oven temperature of about $400{ }^{\circ} \mathrm{C}$, the rare gas atmosphere becomes too warm for effective cluster production. Fig. 2.2 shows the build-up of our ovens: They consist of a cylindrical stainless steel socket for a small crucible and can be mounted into the aggregation tube. The inner wall of the socket is heated by applying current to a heating wire and has a thermocouple attached for temperature control. The heating current is generated by a ac voltage supply operating at $5 \mathrm{VAC}$ to $20 \mathrm{VAC}$ and is regulated by 
a Watlow Series 935A temperature controller. It reads a temperature from a sensor input and switches the current accordingly to reach the specified temperature.
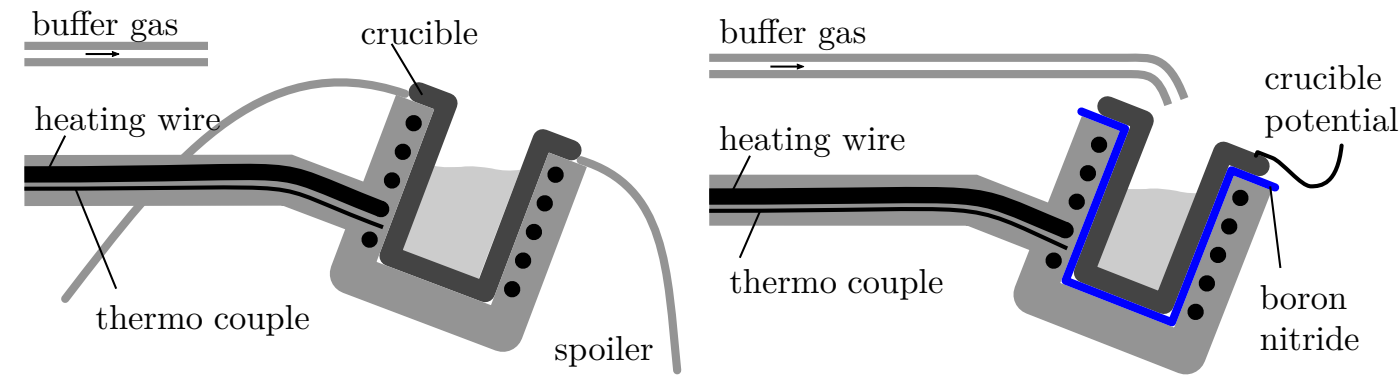

Figure 2.2.: Schematic of the ovens used for evaporation of low melting materials. Left panel: the version used mainly in our setup. Right panel: alternative version with electrically isolated crucible.

Two variations of the oven exist. The first, which is mainly used in this setup, has the rare gas inlet in the back of the aggregation tube and a spoiler to conduct the gas flow past the opening of the crucible (left panel, fig. 2.2). A discharge ring near the aperture of the aggregation tube is used to produce a glow discharge in which the clusters get charged. The second variation has an additional layer of boron nitride between the socket and the crucible (right panel, fig. 2.2). Boron nitride is an electric isolator with a good heat conductivity, thus allowing to apply a potential to the crucible itself. It is therefore possible to ignite a hollow cathode discharge in the crucible. Charging particles in this early stage of the cluster formation process can increase the amount of charged clusters produced in the source. But it also increases the chance of impurities in the clusters, if the vacuum of the source is not high enough. Another difference is that the buffer gas is released into the crucible, which also may improve cluster production 1 .

\subsubsection{Source for Liquids}

The oven can also be used to evaporate liquids. The liquid needs then to be cooled down to very low temperatures (well below its freezing point), to prevent evaporation during non-operational hours. During operation the oven is used to rise the temperature of the material to a point at which the vapor density is suitable for cluster production. In our case for water cluster production we used near liquid nitrogen temperature $\left(-198^{\circ} \mathrm{C}\right)$ for storing and around $-40^{\circ} \mathrm{C}$ during operation. The clusters were charged at the discharge ring near the iris.

Although utilizing the oven for water cluster production did work in principle well, it has two serious drawbacks: First the aggregation tube must be kept cooled

\footnotetext{
${ }^{1}$ This has not been investigated quantitatively yet.
} 
all the time, resulting in a high liquid nitrogen consumption. Second the source chamber has to be vented in order to refill the crucible.

To overcome these shortcomings an alternative source for liquids has been developed (fig. 2.3). It consists of a heatable flange and inlet, which is connected to a detachable reservoir outside the chamber. The liquid in the reservoir is at room temperature and an equilibrium vapor density will be reached according to the pressure in the reservoir. The pressure is adjustable by a fine-regulating valve between reservoir and inlet. An additional membrane valve allows to separate the reservoir completely from the source chamber. This makes it possible to refill or change the contents without venting the source chamber. A connector for carrier gas (e.g. He) exists and can be used as an additional parameter for adjusting the vapor-gas mixture. In practice, however, we could not find any significant improvement in source operations and so it was not used further.

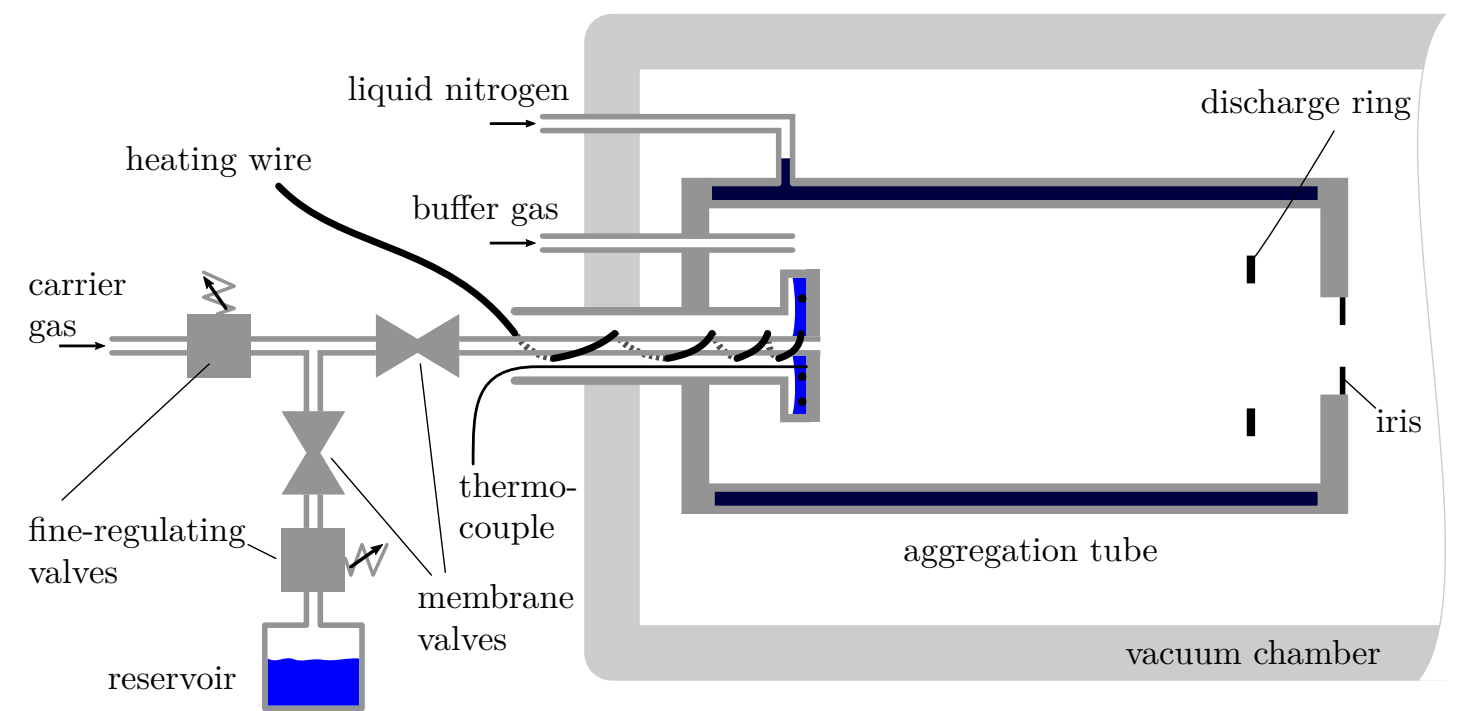

Figure 2.3.: Schematic of the new source used for evaporation of liquids.

The capability to heat the inlet and particularly the flange has turned out to be a necessity to keep the source operational for a reasonable time. Experimentations have shown that without heating the flange the cluster production just stops after some time, presumably due to ice buildup on the inlet area. Inlet and flange are heated by a heating wire, which is wrapped around the tube and is clued in spiral shape to the back of the flange. A thermocouple measures the flange temperature. This way the same power supply and current control unit ${ }^{2}$ can be used as for operating the oven. When producing water clusters the inlet and the flange temperature was kept around $40^{\circ} \mathrm{C}$. For ionization of the clusters, the discharge ring near the iris was utilized, as in the case with the oven.

\footnotetext{
${ }^{2}$ Watlow Series 935 A temperature controller
} 


\subsubsection{Magnetron Sputter Source}

Materials with high melting point can be brought into the gas phase with a magnetron sputter head (fig. 2.4). Although none of the experimental data reported in this work was measured using the magnetron source, a brief description will be given for later reference.

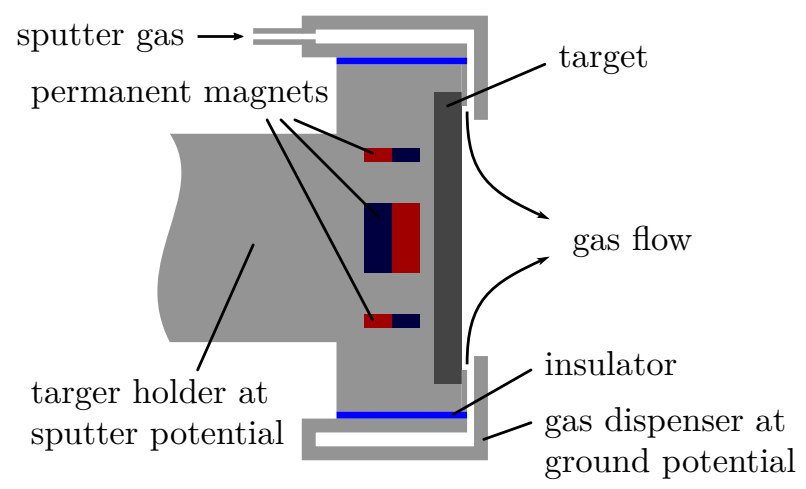

Figure 2.4.: Schematic of the magnetron sputter head.

A high potential is applied between a target (a circular disc of the cluster material) and a sputter gas dispenser mounted close to the target surface. It ignites a plasma in the He-Ar sputter gas flowing near the target surface by glow discharge. Ionized gas particles are accelerated towards the target and eject atoms from its surface upon impact. Circularly arranged permanent magnets in the socket force the accelerated gas ions on a helical trajectory, thus focusing them on a defined region of the surface ("racetrack"). The longer flight path also leads to a higher amount of ionizing collisions with neutral gas particles, which increases the sputter efficiency. The ejected atoms form a dense enough vapor for cluster formation. A large amount of the clusters are already ionized, so that further ionization is not necessary.

The bombardment by the gas ions heats the target considerably. Since the sputter head in our machine is not cooled additionally, the sputter source can only be utilized with target materials, whose melting point is high enough to remain solid under sputter operation.

\subsection{Ion Optics}

The trajectory of charged particles can be controlled and manipulated by forces they undergo by passing electrical fields. These are generated by single electrodes or arrays of electrodes forming together ion optical elements. The potentials applied to the electrodes can be either static or pulsed direct current (dc) potentials or radio frequency (rf) alternating current (ac) potentials. 
Static potential ion optics produce generally symmetric fields (not necessarily in spatial context) in respect to the ion flight path. Consequently they are energy conservative and are used mainly to change the direction of the ions without altering their energy. Examples are the lenses (Einzel lens), the deflectors and reflectors used in our setup.

Pulsed ion optics can be switched to a different static potential for a certain time interval. By this means one can realize two state elements like gates which are transparent for ions only for a given time. The mass gate and the trap exit electrode belong to this category. Another applications of pulsed potentials is altering the energy of ions. When switched while the ions are within the ion optic, they see different potentials when entering and when leaving. Hence they can be accelerated or decelerated.

Some ion optical elements like ion guides or traps can not be realized with static potentials, because they require real potential minimums in two or three dimension. With fast oscillating fields (inhomogeneous radio frequency fields) it is, however, possible to create effective potentials, which, averaged over time, possess those properties. Quadrupole mass filter and Paul trap are prominent examples for the use of rf fields.

A quadrupole mass filter consists of four parallel rod electrodes, of which the diametrically opposed electrodes form a pair. Each pair is connected to a dc potential and an ac potential with opposite polarity respectively: $\phi_{0}= \pm(U+V \cos \omega t)$. Ions entering the mass filter undergo at any point in time an attractive force between one pair of electrodes and a deflective one between the other, whereby the direction of the force changes with every half-period of the ac frequency. Depending on the dc, ac voltages $U, V$, the frequency $\omega$ and the ion mass, this leads to an oscillatory motion of the ions with an amplitude which is either limited or exponentially rising. The exact relations are given by the solutions of the equations of motion (Mathieu equations) [21].

With the correct choice of voltages $U, V$ and frequency $\omega$, ions of a certain mass or even a mass range can pass the mass filter and thus are effectively contained in two dimension. This concept can be extended to multipoles consisting of $2 n(n>2)$ parallel cylindrical rods with diameter $d$ equally positioned on a circle of radius $r_{0}$. The electrodes are connected alternately to voltages of opposite polarity, so that $n$ are connected to the same voltage supply. A full review of the principles and applications of inhomogeneous rf multipole fields has been published by Gerlich 23. The following is limited to summarize the fundamental aspects relevant to our setup.

Analogous to the quadrupole exist parameter sets, which stabilize the ion trajectories for a certain mass range. However, the stability parameters are not analytically calculable as in the case of the quadrupole. Under stable conditions the ions oscillate with the frequency of the rf-field with an amplitude depending on the charge state and mass but have a time-averaged trajectory along the center of the electrode circle. If this drift motion is slow compared to the micro-motion 
induced by the rf, the equation of motion can be split into two parts (adiabatic approximation): the time-averaged drift motion in an effective potential and the quiver motion [23].

Under conditions consistent with the adiabatic approximation, the (time-averaged) trajectory of ions in a time-dependent rf-field $E_{0} \cos \omega t$ can be described by its time-independent effective potential: $V_{\text {eff }}=q^{2} E_{0}^{2} /\left(4 m \omega^{2}\right)$. The shape of the effective potential of a multipole rf-field in radial direction depends on the number of poles $N_{\text {pol }}=2 n$ and their distance from the axis $r_{0}[23]$ :

$$
V_{e f f}(r) \propto \frac{N_{p o l}^{2}}{r_{0}^{2}}\left(\frac{r}{r_{0}}\right)^{N_{p o l}-2} .
$$

From (2.1) follows that the potential rises the steeper the more poles are used, leaving an increasingly field-free space in the center. In fig. 2.5 the relative potentials $V_{\text {eff }}(r) / V_{\text {eff }}\left(r_{0}\right)$ for $4,6,12$, and 22 poles are plotted. A hexa- and dodecapole (blue lines) are used as ion guide and trap respectively in our setup. Quadrupole and 22-pole (black lines) are included for comparison ${ }^{3}$

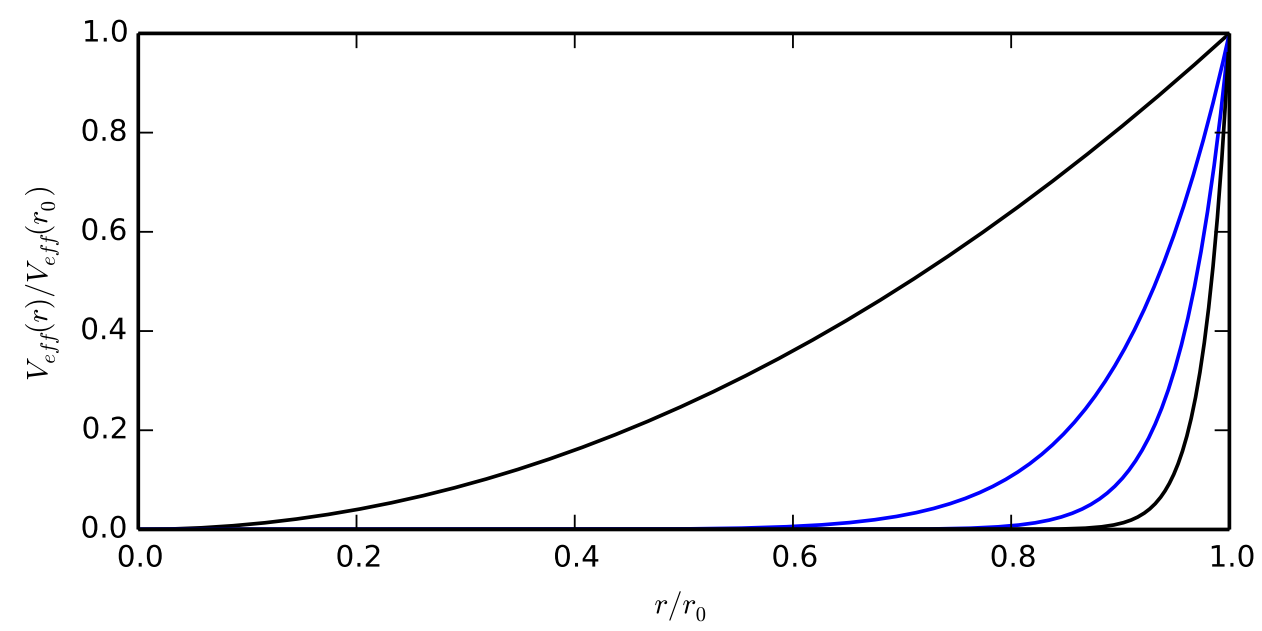

Figure 2.5.: Effective potentials in radial direction of a quadrupole, hexapole, dodecapole and 22-pole (from left to right).

Whereas the harmonic potential of a quadrupole provides for good ion alignment near the axis, the larger field-free space of higher order multipoles is better suited for the use as ion guides and traps. Besides the larger trapping volume the effective potential of higher order multipole fields is less sensitive to misalignment of the electrodes by mechanical displacement. Circular electrodes are a good enough approximation of the ideally hyperbolic ones and have no significant impact on the

\footnotetext{
${ }^{3}$ The 22-pole trap was the first published multipole rf ion trap[24].
} 
guiding efficiency ${ }^{4}$. The best approximation is reached by choosing the electrode radius $r_{\text {rod }}$ according to the following rule:

$$
r_{\text {rod }}=\frac{r_{0}}{n-1}
$$

The radio frequency of the guide and trap ranges in our setup between $0.5 \mathrm{kHz}$ and $1.8 \mathrm{kHz}$. The amplitude voltages depend on the method of $\mathrm{rf}$ production. The guide is generally connected to a home-made rf generator [46], which is capable to drive it off-resonantly. It may output amplitudes up to $300 \mathrm{~V}$. The trap rods are usually connected to LC-resonator (and acting as capacitor), which is driven resonantly by a square-wave voltage. The resulting amplitudes range from few volt to a few ten volt.

\subsection{Ion Optics Between Source and Thermalization Stage}

The output of the cluster source consists of a mixture of buffer gas, neutral, positively and negatively charged clusters. A radio frequency (rf) ion guide collimates and transports the charged clusters into the next vacuum chamber. The 6 rod electrodes of the guide are connected alternately to ac voltages of opposite polarity. Averaged over time the ac potentials generate an effective cylindrical confinement of the ions along the symmetry axis of the guide. The amplitude and the frequency of the ac potential determines the mass-over-charge ratio which can be contained by the guidance field. This means that only clusters with masses within a certain interval pass the guide. The potential at the center is given by the reference voltage of the ac voltage. It can be used to add an additional momentum to the clusters. The guide is mounted in a way that its axis is tilted by about $15^{\circ}$ in respect to the propagation direction of the clusters coming from the source. This way only charged clusters reach the next chamber while the neutrals are deposited on the chamber walls. Near half the length of the guide, at the interconnection of the vacuum chambers, is an aperture electrode placed, which adds a further parameter for modifying the guiding potential. Additionally, its small opening also minimizes the gas flow into the next chamber.

After leaving the guide the ions enter a quadrupole ion deflector. It deflects the beam by about $105^{\circ}$ to adapt it to the l-shaped arrangement of the vacuum chambers. The ion deflector consists of two pairs of rod electrodes with a quarter circle base, to which a static potential of opposite polarity is applied. Additional electrodes at the entrance and exit apertures can be used to focus the beam and correct for the cylindrical aberration inherent to this type of deflector. Ions with

\footnotetext{
${ }^{4} \mathrm{~A}$ quadrupole gains its highest mass selectivity when hyperbolic electrodes used.
} 
different charge polarities are deflected in opposite directions, so that either cluster anions or cations proceed to the trap.

\subsection{Ion Trap and Thermalization Stage}

The ion trap and thermalization stage is used to prepare high intensity ion packages for the time of flight mass spectrometer/-selector and to set the mean temperature of the clusters. The clusters arriving at the trap have a very broad temperature distribution [60], which presumably originates partially from the growth process in the source and partially from collisions with gas atoms in the guide in the high pressure of the source chamber. Since all measurements are realized with ensembles of clusters, no meaningful average temperature can be assigned without proper thermalization.

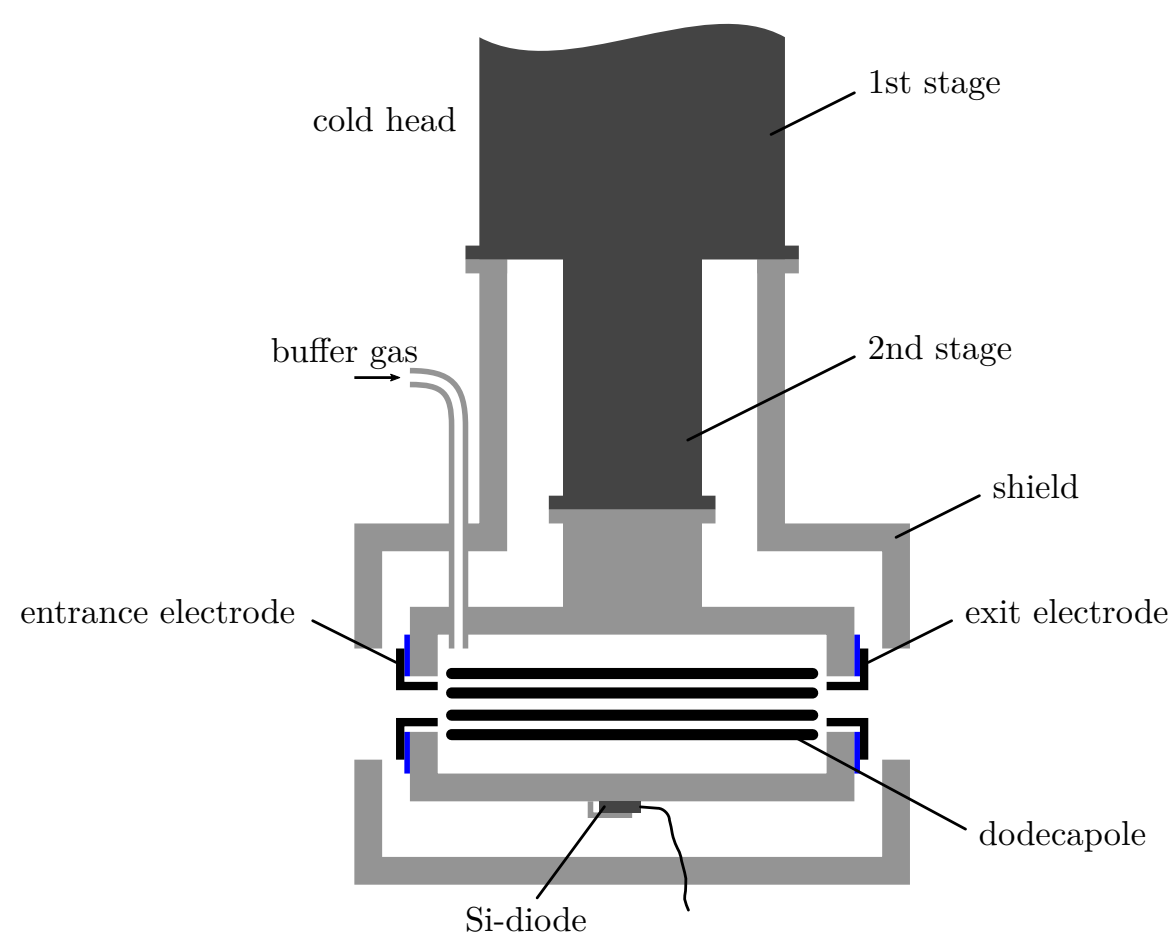

Figure 2.6.: Schematic of the rf ion trap and the thermalization stage.

A schematic sketch of the setup is given in fig. 2.6. The trap is based on the design of the 22-pole rf trap published by Gerlich 24], but utilizes 12 stainless steel rod electrodes $5^{5}$ to generate a cylindrical guidance field[46]. Potentials on the entrance

${ }^{5} l_{\text {rod }}=44 \mathrm{~mm}, r_{\text {rod }}=2 \mathrm{~mm}, r_{0}=5.3 \mathrm{~mm}$ 
and exit electrodes provide for ion containment along the propagation axis. The exit electrode can be switched to an attractive potential to extract ion packages from the trap.

The trap is fitted into a hollow copper cube with a gas inlet and openings for the entrance and exit apertures. The cube is mounted to the second stage of the cold head of a closed cycle liquid helium refrigeration system. A silicon diode is attached to one of the walls to determine the cube temperature by 4-point resistance measurement. A copper shield affixed to the first stage of the cold head encloses the cube and provides additional thermal shielding. The inner copper cube can be cooled down to $10 \mathrm{~K}$ by the second stage of the cold head. Some parts of the cube are electrically isolated from each other because they are connected to electrodes of the trap. They are thermally coupled to the cube base by sapphire plates, boron nitride discs and quartz rods. Although good heat conductors they may prevent this parts from reaching an equally low temperature as the parts directly affixed to the cold head.

During operation the inner copper cube containing the trap is flooded with $\mathrm{He}$ buffer gas through the gas inlet. It leaves the cube through the entrance and exit apertures. Under equilibrium condition the pressure it the trap is few order of magnitudes higher (around $2 \times 10^{-3}$ mbar) than the pressure in the cube chamber (around $4 \times 10^{-5} \mathrm{mbar}$ ). During the time the clusters stay in the trap, they collide many times with buffer gas atoms. That way a heat transfer between clusters and trap wall is established and the clusters will, after a sufficient number of collisions, adopt the temperature of the enclosing cube. Through collisions the clusters also loose kinetic energy. This highly increases the trapping efficiency, because it prevents those clusters from leaving the containment field of the trap. While the ion beam from the source is continuous, only a small amount of ions leave the trap with the extraction pulse. Therefore the trap can be assumed to be filled with ions to the space charge limit.

\subsubsection{Thermalization of clusters}

The main heat transfer mechanism in the trap are collisions of the clusters with buffer gas atoms. A good thermalization of the cluster ensemble is reached, if each cluster undergoes a high enough number of collisions. The required number of collision is not easily specified. Investigations by experiments and simulations have shown [34, 60] that the number of collisions not only depends on the temperature difference between the initial and desired cluster temperature $\Delta T=\left|T_{i}-T_{f}\right|$, but also on the cluster material, the cluster size, and the targeted final temperature $T_{f}$.

The number of the collisions depends mainly on the temperature, the pressure inside the trap enclosure, the cluster radius and the average time $\langle t\rangle$ the clusters stay in the trap. $\langle t\rangle$ in turn depends on the frequency and duration of the extraction pulse. Experiments on a structurally similar, but longer rf trap have shown 60 
that an extraction pulse of several hundred ms is needed for emptying the trap completely. Since the pulse frequency lies between $20 \mathrm{~Hz}$ and $100 \mathrm{~Hz}$, it follows that a lower limit to the average storage time is given by the frequency: $50 \mathrm{~ms}$ to $10 \mathrm{~ms}$. The actual extraction pulse duration is much shorter (e.g. about $45 \mu$ s for a large water cluster). To estimate the storage time with short extraction pulse lengths, the number of extractions cycles required to empty the trap to $1 / e$ of its original content has been measured [34, 60]. The number ranges from 7 to nearly 40 . If we choose 5 cycles as save minimum, then we end up with $\langle t\rangle=166 \mathrm{~ms}$ using an extraction frequency of $30 \mathrm{~Hz}$. The minimal thermalization time of an ensemble $\left(10^{6}\right)$ of $\left(\mathrm{H}_{2} \mathrm{O}\right)_{48}{ }^{-}$and $\left(\mathrm{H}_{2} \mathrm{O}\right)_{118}{ }^{-}$clusters has been determined by simulations for various start and target temperatures 60]. The minimal time depends heavily on the situation and ranges from $3.5 \mathrm{~ms}$ to $15 \mathrm{~ms}$.

Although the details of a complete thermalization of clusters in a rf trap depend on many factors and can not be generalized, it is safe to assume that the average storage time for most use cases is much longer than the minimal thermalization time. For the trap used in our setup, this has been verified for sodium clusters by means of photofragmentation measurements of $\mathrm{K}_{92}{ }^{+}$and $\mathrm{K}_{139}{ }^{+}[55]$. The experiment showed that the cluster ensemble could be thermalized to a common temperature for all trap wall temperatures 5 . The mean temperature was in good accordance (about $\pm 5 \mathrm{~K}$ ) with the trap wall temperature between $300 \mathrm{~K}$ and $40 \mathrm{~K}$. For lower trap wall temperatures the clusters could be cooled further, but the cluster temperature deviated increasingly from the trap temperature. Photofragmentation spectroscopy allows, however, only indirect determination of the cluster temperature $7^{7}$ and especially low temperature determination relies on hard to verify assumption of the Debye temperature of the specific cluster. For the very low temperatures it is reasonable to assume that not all parts of the trap housing reach the measured temperature and therefore the He buffer gas neither.

The analysis of the photoelectron spectra presented in this work does not require highly accurate temperature measurements. In the following we will assume therefore that the cluster temperature equals roughly the trap wall temperature and the meaning of both will be swapped freely.

\subsection{Mass Selection and Spectroscopy}

Mass analysis and selection is done with a time-of-flight (TOF) mass spectrometer. It measures the time charged particles need to pass a given drift length, after having been accelerated in an electric field: In a potential $U$ a cluster with charge $q$ gains the energy $q U$. The kinetic energy of a previously stationary cluster with mass $m$

\footnotetext{
${ }^{6}$ The characteristic modulation of the fragment spectrum vanishes, if the temperature variation within the ensemble is to high.

${ }^{7}$ excluding comparison of spectra with reference spectra of known temperature
} 
will then equal the gained energy after leaving the potential: $E_{k i n}=\frac{m}{2} v^{2}=q U$. The time $t_{f}$ a cluster needs to traverse a field free drift length $l$ is given by

$$
t_{f}=\sqrt{\frac{m}{2} \frac{l^{2}}{q U}} \propto \sqrt{\frac{m}{q}} .
$$

The flight time $t_{f}$ is proportional to the mass-over-charge ratio or, in the case of singly charged clusters $(q=1)$, to the mass only.

In order to achieve high mass resolution, that means a sufficient spatial separation of different masses, long flight times are necessary. This implies, however, that a flight time spread of a package of ions of the same mass must be kept at a minimum. The two main reasons for flight time broadening are a initial spatial and/or velocity distribution of the ions in the acceleration region. To overcome those effects, one uses nowadays often a combination [9] of a two stage ion accelerator, introduced by Wiley and McLaren [81, and reflectrons, based on the work of Mamyrin et al. [58. The general idea is to compensate a initial spatial distribution with a two stage accelerator and use its space focus as a ion source for a reflectron, which can then be used to compensate the kinetic energy spread of the ions.

A two stage accelerator consists of three electrodes $\left(U_{1}, U_{2}, U_{t o f}\right)$ mounted at spacings $d_{1}, d_{2}$, which produce the two accelerating fields. By choosing the parameters accordingly, one can either specify the length $l_{f}$ of the first order space focus 8 [81] or achieve a second order space focus at fixed geometries $\left(d_{1}, d_{2}, l_{f}\right)$ [79]. Flight time variations due to a spatial distribution $\Delta x$ of ions in the accelerator are intrinsically compensated in the (1st order) space focus: Ions starting with a negative offset in respect to a central position, also gain higher kinetic energies due to their starting point being in a higher potential region. So all ions reach the space focus at the same time but with different kinetic energies. This is only true for stationary ions in the accelerator. Under real experimental conditions the ions will always have a certain velocity or kinetic energy distribution, which leads to packet broadening at the focal point. The packet length $\Delta l$ at the focal point $l_{f}$ for an ion packet with an initial energy spread of $q \Delta U$ amounts to $\Delta l \approx l_{f} \sqrt{\Delta U / U}$, where $q U$ is the ion energy in the drift space. A short package length thus requires a short focus length or a strong (first stage) acceleration field. For long flight paths, the first stage acceleration field has to be weak however, so that in both cases the mass resolution suffers.

The common solution is to use a reflectron to correct the kinetic energy broadening. A reflectron is an electro-static ion mirror consisting of either a single reflection field or of two fields, a stronger accelerating/decelerating field and a weaker reflection field. The reflection field is usually generated by a stack of ring-electrodes

\footnotetext{
${ }^{8}$ In a development of the flight time in terms of the energy spread $\Delta U$ and a spatial displacement $\Delta x$ respectively, the linear term vanishes (first order) or the linear and quadratic term vanishes (second order).
} 
linked by a resistive chain. In case of a two stage reflectron an additional ringelectrode in front of the stack generates the steep deceleration/acceleration field. The energy focusing effect of a reflectron results from the deeper penetration of higher kinetic energy ions into the reflection field. With the appropriate potentials and geometry the shorter flight time of high energy ions in the field-free drift region is compensated for by their longer turn-around time within the reflector.

The combination of a two stage accelerator with a reflectron allows to adjust the accelerator to minimize the flight time broadening stemming from a initial spatial or velocity distribution of the ions without the need for a long flight path to the first focus point. Flight-time broadening in the drift region behind the space focus caused by difference in the kinetic energies are then compensated by a reflectron while simultaneously lengthen the flight path. This way long flight paths and correspondingly high mass resolution can be realized.

\subsubsection{Setup}

In our setup (cf. fig. 2.1) we use a two stage accelerator with $U_{1}=1200 \mathrm{~V}$, $U_{2}=1000 \mathrm{~V}$, and $U_{\text {tof }}$ at ground potential together with two reflectrons. The second order space focus of the accelerator is mapped by the first reflector to the second focus point. Here is the mass gate located? The second focus point is mapped by the second reflector to the third focus point, where the ion detector is mounted. Ion beam deflectors as well as a Einzel lens installed closely behind the accelerator allow for horizontal and vertical beam corrections and help to concentrate the ion packages. Additional lenses after the second reflector and close to the decelerator can be used to compensate for beam divergence. The decelerator near the third focus is not used for mass spectroscopy, but for decelerating the ions for photoelectron spectroscopy and for shifting the focus point from the ion detector to the interaction region of the photoelectron spectrometer (cf. sec. 2.6). The mass resolution achieved with this setup amounts to $m / \Delta m \approx 4000$.

\subsubsection{Mass Filter}

The mass filter is a switchable ion deflector positioned in the flight path of ions. When switched off it is transparent for ions, otherwise they are deflected by a small angel and consequently deviate far enough from their predetermined flight path to miss the detector. It is located near a focus point of the TOF, so that ions of different masses pass at separate times. For mass selection the mass gate is switched into transmissive mode for a short time interval. That way only ions with a specific flight time, i.e. with a specific mass, can pass the gate.

\footnotetext{
${ }^{9}$ And a laser ion beam interaction zone used for photofragmentation experiments.
} 
To gain a high mass selectivity the deflection field of the gate must fulfill the following requirements [70] in relation to the temporal and spacial separation of the passing ion packages: (i) short rise and fall times as well as adequate pulse duration, and (ii) low field penetration along the flight path.

Our mass gate is based on the modified version[70] of a interleaved comb device originally developed by Bradbury and Nielsen 10]. It consists of two sets of thin wires (diameter $25 \mu \mathrm{m}$ ) mounted parallel to each other (spacing $250 \mu \mathrm{m}$ ) and perpendicular to the ion beam direction. The wires of each set are mounted alternately and can be switched between $\pm 200 \mathrm{~V}$ respectively and $U_{\text {tof }}$ by a high voltage pushpull switch. The deployed switches can realize pulse widths down to $100 \mathrm{~ns}$ with rise/fall times of about $15 \mathrm{~ns}$. The field penetration is proportional to the inter-wire distance and can be estimated[70] as approximately $1.5 \times 250 \mu \mathrm{m}=375 \mu \mathrm{m}$.

Assuming an actual minimal pulse width of about $100 \mathrm{~ns}$ the intrinsic selectivity amounts to $m / \Delta m \approx 2000$, based on an average kinetic energy of $1100 \mathrm{eV}$ of the cluster ions and the drift length available in our setup. The selectivity is high enough to single out even large cluster sizes (up to $\approx 3500$ ), if they are monoisotopic. For clusters of multi-isotopic material or when impurities need to be cut off, the maximum selectable size decreases accordingly.

\subsubsection{Ion Detector}

The actual flight time measurement is done by detecting the cluster ions at the end of the drift tube. This requires a detector which can process a wide range of ion velocities, in order to be able to detect a wide range of cluster masses. In our setup we deploy a so called Even-cup ion detector [4] which fulfills these requirements: A small stainless steel cup (fig. 2.7) is maintained at a high potential $(25 \mathrm{kV}$ to $30 \mathrm{kV}$ ) in respect to the flight tube $\left(U_{t o f}\right)$. The cluster ions are accelerated into it and hit the conversion dynode with high kinetic energies, where they release secondary electrons. The entry of the cup is closed up with a fine mesh, leaving its interior field free. A small opening on one side leads to the scintillator, which is kept at a positive $20 \mathrm{kV}$ potential in respect to the cup. The emitted electrons from the dynode are accelerated towards the scintillator and are converted into photons, which are guided by a light transmission bar to a photomultiplier.

\subsection{Photoelectron Spectroscopy}

Photoelectron spectroscopy is a well established experimental technique for analyzing the electronic structure of clusters. It measures the kinetic energy $E_{k i n}$ of electrons emitted from the clusters by excitation with laser photons of energy $h \nu$. The energy difference is interpreted as the binding energy of the electron: $E_{b i n}=h \nu-E_{k i n}$. 


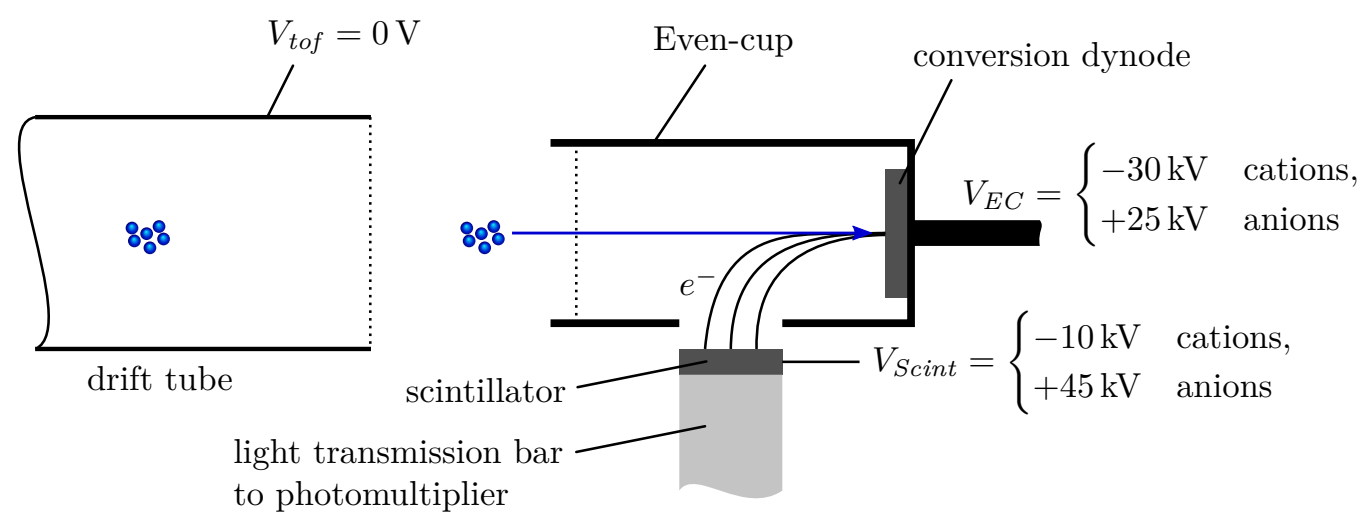

Figure 2.7.: Schematic of the Even-cup ion detector. Potentials given as used in our setup.

One fundamental issue of photoelectron spectroscopy on pulsed cluster beams is the low electron signal. The reason for this lies in the low number of clusters in each ion package. It has been estimated [15] that the laser interaction region of a spectrometer contains some thousand cluster ions, if the beam intensity is high. Since the detachment volume must be kept small $\left(<1 \mathrm{~cm}^{3}\right)$, an upper limit of $10^{5}$ is given because the space-charge starts to effect photoelectron energies. The number of clusters gives an upper limit of photoelectrons per duty cycle. Additionally the photoelectrons are emitted generally in all directions in space (especially, when using unpolarized photons). A spectrometer with a high electron collection efficiency must therefore cover as large a solid angle as possible.

The magnetic bottle time-of-flight photoelectron spectrometer is particularly suited in this respect and has a high electron yield. It uses a strong and highly divergent magnetic field in the region where clusters are irradiated by laser and a weak static field in the drift tube. In the strong magnetic field the emitted photoelectrons are constrained by the Lorentz force to spiral along the field lines in the direction of the lower field strength to the electron detector. Consequently the "bottleneck" of the magnetic bottle works as an electron mirror, so that almost all emitted electrons are captured ( $>98 \%$ are possible[15]). The measured quantity is the time $t$ it takes an electron to reach the detector after photodetachment from the cluster. For a known trajectory length $l$ between detachment point and detector the kinetic energy of electron can then be calculated by:

$$
E_{k i n}=\frac{m_{e}}{2}\left(\frac{l}{t}\right)^{2}
$$

In practice, however, the trajectory is unknown and can only be approximated by the known distance between detachment region and electron detector. The measured flight time correlates therefore with the longitudinal component of the 
electron velocity in respect to the drift tube axis: $t=l / v_{\text {long }}$. So the actual kinetic energy is given by eq. (2.4) only, as long as $v_{\text {long }} \approx v$ is fulfilled.

Kruit and Read have shown[50] that the special field form of the magnetic bottle parallelizes the trajectories of the electrons by transforming the transversal component of the initial velocity almost completely into longitudinal movement. The absolute value of the velocity is kept constant due to angular momentum conservation. The longitudinal component of the velocity in the drift tube is then given by:

$$
v_{\text {long }}=v\left(1-\frac{B_{f}}{B_{i}} \sin ^{2} \Theta_{i}\right)^{\frac{1}{2}},
$$

with $v$ denoting the absolute value of the velocity, $B_{i}$ and $B_{f}$ the initial (at the detachment region) and final magnetic field strength (in the drift tube) and $\Theta_{i}$ the emission angle in respect to the drift tube axis. For the angular momentum to be a conserved quantity, the variation of the magnetic field along the drift tube must be adiabatic, i.e. the change in field strength experienced by an electron during one revolution of the helical motion must be small.

From eq. 2.5) follows that for a high field ratio $B_{i} / B_{f}$ the longitudinal velocity $v_{\text {long }}$ corresponds in good approximation with $v$ and $(2.4)$ indeed gives the kinetic energy of the electron. The ratio of the magnetic field strengths not only determines the grade of parallelization, but also effects the time the electron trajectories need until they reach maximum parallelization in the weak, constant guiding field. The alignment and turn-around time can be reduced by choosing the divergent part of the magnetic field comparably short in respect to the constant part. The intrinsic limit of the resolution of the spectrometer can be approximated as

$$
\frac{E}{d E} \approx \frac{B_{i}}{B_{f}}
$$

It amounts to 100 in our setup.

Other sources for a flight time spread are the finite pulse width of the laser (e.g. $10 \mathrm{~ns}$ to $20 \mathrm{~ns}$ for our excimer lasers) and the finite volume in which electron emission takes place. A different emission origin causes the electrons to spiral around different field lines, which in turn leads to slightly different trajectories. Both effects are proportionally small.

The by far largest time spread stems from the motion of the cluster ions in the interaction zone (Doppler broadening) and is not inherent in the workings of the magnetic bottle. In the laboratory frame of the spectrometer the electron velocity can reach values between $v=v_{e} \pm v_{c}$, depending on the direction in which the electron is emitted $\left(v_{e}\right.$ : electron velocity in the center-of-mass system of its cluster, $v_{c}$ : cluster velocity, and in general $v_{c}<v_{e}$ ). In terms of energy the spread amounts to

$$
\Delta E \approx 4 \sqrt{\frac{m_{e}}{m_{c}} E_{e} E_{c}} .
$$


With a pulsed ion decelerator it is possible to reduce the cluster energy to nearly $10 \mathrm{eV}$. Deceleration is limited, however, because with lower energies the ion package drifts further apart, thus reducing the photoelectron signal.

Another cause of photoelectron signal deterioration are background electrons. They originate from residual gas in the beam path or are ejected from surfaces enclosing the laser cluster beam interaction zone by stray light. The high energy photons of UV lasers are capable of detaching electrons from metal surfaces (e.g. the work function of copper: $4.48 \mathrm{eV}$ to $4.98 \mathrm{eV}[44]$ ), especially if they contain contaminants, which further reduce the work function. The workings of the magnetic bottle, however, strongly diminish the amount of detected electrons originating far off the spectrometers axis: The coupling of the electron trajectories to field lines and the shape of the magnetic field effectuates that only a small area centered around the spectrometers axis is mapped to detector.

A further source of background electrons, which can even cause "false" peaks in the spectrum, is the so called ion feedback in certain types of electron multipliers like single channel multipliers: residual gas or adsorbed atoms at the channel surface can be ionized by the electron avalanches in the multiplier and are accelerated by the channel bias towards the entrance. If they gain enough energy before hitting the channel wall, they can initiate a second electron avalanche.

\subsubsection{Setup}

In fig. 2.8 the setup of the magnetic bottle type photoelectron spectrometer used in our experiment is shown schematically. A permanent magnet beneath the laser interaction zone generates a strong magnetic field, which drops over a short distance to blend with the homogeneous field of the large solenoid of the drift tube. To smooth the transition from high to low field strengths an additional, smaller solenoid is used between interaction zone and drift tube. Interfering magnetic fields, like the earth magnetic field, are either compensated by two pairs of coils mounted perpendicular to the homogeneous field of the drift tube or shielded by wrapping the drift tube vacuum chamber in Mu-metal sheets. An inner drift tube made from copper is mounted into the vacuum chamber to decrease perturbations of the electron energies by inhomogeneous electrical fields. The inside is coated with graphite to align the surface work functions and to protect it from oxidation and coverage of residual oil in the vacuum. The drift length amounts to $1.6 \mathrm{~m}$.

After passing the drift tube the electrons are detected by a Channeltron electron multiplier (CEM)[14]. A CEM is a continuous dynode multiplier consisting of a funnel-shaped glass body coated interiorly with a semi-conducting layer. A positive bias accelerates incoming electrons down the channel and every time they hit a wall a cascade of secondary electrons is generating. The output signal of the CEM is 


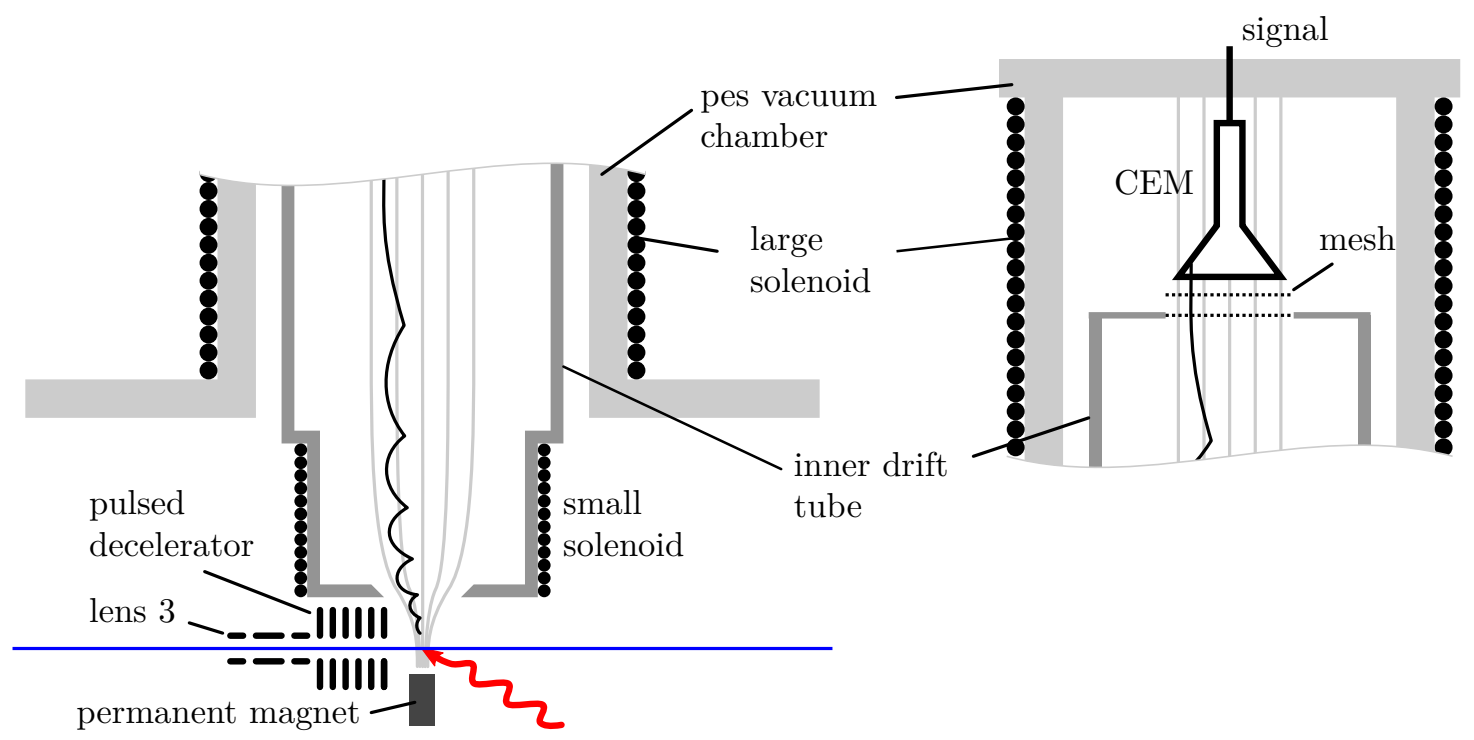

Figure 2.8.: Schematic of the lower part of the photoelectron spectrometer (left), which generates the "bottleneck" of the magnetic bottle and the upper part (right), housing the photoelectron detector.

amplified by a fast rise-time, bipolar preamplifier ${ }^{10}$

A stack of apertures is mounted near the crossing point of laser and cluster beam into the path of the incoming laser beam. It confines the beam to a fixed diameter in the interaction zone to reduce the amount of background electrons originating from the surfaces of the spectrometer. The beam exit window is slightly tilted to prevent reflecting laser light from reentering the interaction zone.

The cluster decelerator (cf. left panel fig. 2.8) consists of 6 ring electrodes connected together by a resistive chain [46]. The last electrode is kept at TOF potential $\left(U_{\text {tof }}=0 \mathrm{~V}\right.$ in our case), whereas the first electrode can be switched to a high attractive potential (around $2 \mathrm{kV}$ ). When switched on, a homogeneous deceleration field is generated inside the decelerator. To minimize distortions by field inhomogeneities in fringe areas the potentials is switched on and off while the cluster ions are located well inside the decelerator. According to simulations [46] the clusters initial energy of about $1100 \mathrm{eV}$ can be reduced to nearly $10 \mathrm{eV}$. Since the space focus of the second reflector of the TOF is placed behind the laser interaction zone, ions with higher velocities are still in the rear of the package. The deceleration field shifts the focus point in direction of the interaction zone and thus shortens the longitudinal extent of the ion package. The Einzel lens (lens 3) in front of the decelerator can be used to focus the cluster package transversally. The resolution of the spectrometer under operational conditions amounts to $E_{k i n} / \Delta E_{k i n} \approx 42$ or $2.3 \%$.

${ }^{10}$ Phillips Scientific Model 6954 


\subsubsection{Influence of the cluster temperature}

For some cluster materials the photoelectron spectra differ for different cluster temperatures. As a general trend the spectra of clusters with higher temperatures show broader peaks with fewer features as compared to lower temperature clusters. The apparently lower resolution is, however, not caused by the resolving limits of the spectrometer, but has its origin in the less sharply defined energies of the electrons in warm clusters (vibrational broadening, sometimes isomerization). Fig. 2.9 demonstrates the effect of the cluster temperature on the PES of $\mathrm{Au}_{12}{ }^{-}$clusters. While the device specific resolution stays the same for all spectra, the spectrum of
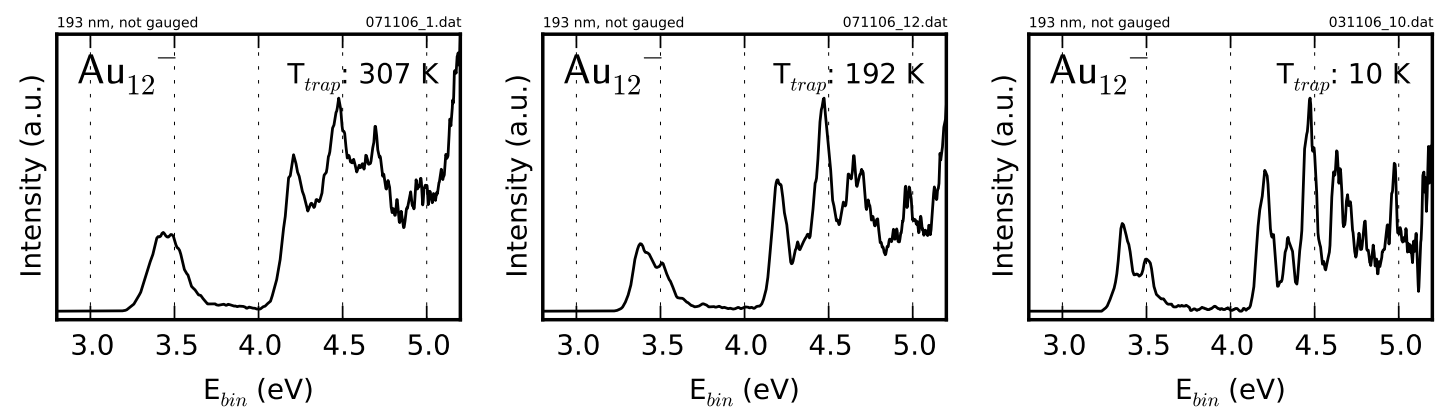

Figure 2.9.: Photoelectron spectra of gold cluster anions at different cluster temperatures. The spectrum grows reacher in features and details with decreasing temperature.

the warm clusters shows only the major features. With decreasing temperature the peaks of the spectra grow sharper and more and more of the minor features and its "fine" structure are revealed.

If the temperature influences the resolution at all and at which temperatures the changes occur depends on the cluster material. The Debye temperature $\left(\Theta_{D}\right)$ of the material can be used to estimate the impact of the temperature qualitatively ${ }^{11}$; For gold $\left(\Theta_{D}=165\right)$ 44 and sodium $\left(\Theta_{D}=158\right)$ the resolution improves strongly between $300 \mathrm{~K}$ and $80 \mathrm{~K}$ and slightly between $80 \mathrm{~K}$ and $10 \mathrm{~K}$. For potassium $\left(\Theta_{D}=\right.$ 91) the resolution improves considerably even between $80 \mathrm{~K}$ and $10 \mathrm{~K}$. Copper $\left(\Theta_{D}=343\right)$ and silver $\left(\Theta_{D}=225\right)$ show only small enhancement between $300 \mathrm{~K}$ and $80 \mathrm{~K}$.

${ }^{11}$ Based on pes measurements at various temperatures on different clusters conducted with our experiment. 


\subsection{Vacuum System and Laser}

\subsubsection{Vacuum System}

The successful operation of the experiment requires that the collisions between clusters and residual gas are kept at a minimum. This has various reasons: Impurities in the vacuum can be picked up by the clusters and hence reduce the amount of pure clusters available for measurements. The workings of the spectrometers, especially the PES, suppose collision free drift paths. Collisions with buffer gas can also cause the clusters to heat up or even fragment. The vacuum system, consisting of the vacuum chambers, the vacuum and prevacuum pumps, must be geared to provide adequate conditions.

The cluster source, ion optics and spectrometers are mounted in four vacuum chambers, separated by valves into three independent vacuum systems. The pumps and the prevacuum system of each partition is adapted to the pumping requirements of that section. Additionally the separation allows to vent only one part of the experiment, e.g. for maintenance. To minimize the gas flow between the chambers, apertures (diameter between $10 \mathrm{~mm}$ and $80 \mathrm{~mm}$ ) are mounted into the connecting lead-throughs.

The first partition is formed by the source chamber and the cube chamber, which contains the quadrupole deflector, trap and accelerator of the TOF. Both chambers are connected to a $1000 \mathrm{ls}^{-1}$ turbo-molecular (turbo) pump each. The high pumping capacity is necessary to keep up with the high gas flow during source operation. When the source is not in operation, pressures are in the high $10^{-8}$ mbar (source chamber) and low $10^{-7}$ mbar region (cube chamber) respectively. A high vacuum is especially important in the source chamber to minimize the take up of impurities by the clusters, to which they are particular susceptible during the formation process. The source chamber is therefore $\mathrm{CF}^{12}$ sealed except for the maintenance opening, which is sealed by two differentially pumped Viton o-rings. The cube chamber is viton-sealed (hence the higher pressure). During source operation the pressure amounts to $10^{-2}$ mbar in source chamber and $10^{-5}$ mbar in the cube chamber. The gas flow from the source chamber is, despite the small aperture between the chambers, still the main cause for the pressure in the cube chamber, although the buffer gas of the trap also contributes to a small degree.

The prevacuum for the turbo pumps of both chambers is generated by a rotary vane pump. An adsorption filter with activated alumina ${ }^{13}$ beads prevents a contamination of the vacuum with pump oil via back diffusion. When the source is running the filter can be bypassed to allow a higher gas throughput.

\footnotetext{
12"ConFlat" (a registered trademark of Varian, Inc.) is a commonly used designation of a metalto-metal seal, where each face of the two mating flanges has a knife edge cutting into a copper gasket.

${ }^{13}$ aluminum oxide
} 
The second partition is formed by the vacuum chamber housing the ion optics of the mass spectrometer. The third partition consists of the chambers for the photoelectron spectrometer and the Even-cup ion detector. Each partition is pumped by $300 \mathrm{ls}^{-1}$ turbo pump and reaches a pressure in the low $10^{-7} \mathrm{mbar}$ region. To minimize the gas flux from the cube chamber, the connection to the chamber of the mass spectrometer is differentially pumped by a $250 \mathrm{ls}^{-1}$ turbo pump.

The prevacuum for both partitions is generated by a turbo pump $\left(10 \mathrm{ls}^{-1}\right)$ and a pressure triggered dry scroll pump, which evacuates a long part of the prevacuum tube serving as "vacuum buffer" for the turbo pump. The system is designed to keep the vacuum of the spectrometers completely oil free.

The pressure in the vacuum chambers and prevacuum tubes can be monitored by individual gauges of various types (full range, cold cathode, hot cathode, prevacuum).

\subsubsection{Laser}

The laser wave length determines which binding energy range can be probed with the photoelectron spectrometer, and has to be chosen accordingly for each cluster material (and size). Another reason to adapt the laser wave length is the absorption cross section, which may vary for different wave length/cluster material combinations. Sometimes a smaller photon energy is chosen in order to investigate a smaller binding energy range with higher resolution. The following list summarizes the lasers used in our experiment for the various wave lengths, together with the active medium and their typical pulse durations:

- $308.4 \mathrm{~nm}$ (4.02 eV): Lambda Physik LPX 120i, XeCl, pulse width 10 ns.

- $248.4 \mathrm{~nm}(4.99 \mathrm{eV})$ : Lambda Physik OPTex, KrF, pulse length 8 ns.

- $193.3 \mathrm{~nm}(6.42 \mathrm{eV})$ : TuiLaser ExciStar S-200, ArF, pulse width 10 ns.

- $300 \mathrm{~nm}$ to $900 \mathrm{~nm}$ : Lambda Physics FL 3002, dyes, pumped by $308.4 \mathrm{~nm}$ excimer laser (Lambda Physik LPX 120i).

The high energy ultra violet (UV) photons are exclusively produced by excimer lasers, which use temporary rare gas dimers or rare gas halogen complexes as active medium. Photons in the visible spectral range, e.g. $590 \mathrm{~nm}$, can be produced by an excimer pumped dye laser. 


\subsection{Data Acquisition and Analysis}

The voltage output from the photomultiplier (MS) and amplifier (PES) respectively is measured by a $1 \mathrm{GHz}$ digital storage oscilloscope ${ }^{14}$. For a specific time interval (ms: between $1 \mu \mathrm{s}$ and $1 \mathrm{~ms}$; PES: $5 \mu \mathrm{s}$ or $10 \mu \mathrm{s}$ ) the voltage signal is added up and averaged over several thousand measurement cycles. In the case of a mass spectrum the interval starts at the switching point of the accelerators of the mass spectrometer (trigger signal for the high voltage switch). For a photoelectron spectrum the start time gets triggered by a voltage pulse from a photo diode measuring the diffuse reflection of the laser pulse. The recorded spectrum can be read out from the oscilloscope for further processing. The raw data consists of a list of average voltages per time slot (2500 to 25000 data points; $2 \mathrm{~ns}$ for an interval of few $\mu \mathrm{s}$ else (interval)/25000). The time of each data point can be calculated from its index $i$ and the length of the time slot $\Delta t$ by $t=i \Delta t+\Delta t_{\text {trigger }}$, with $\Delta t_{\text {trigger }} \geq 0$ a possible trigger offset. All further evaluation steps are performed with a newly written software for cluster spectra analysis (cf. appendix C).

\subsubsection{Mass Spectra and Calibration}

The mass of a cluster with a measured flight time $t_{f}$ can be derived from (2.3) (cf. sec. 2.5):

$$
m=2 q U\left(\frac{t_{f}}{l}\right)^{2}
$$

The exact values for $U$ and $l$, however, can not be determined easily with sufficient accuracy. One way to work around this problem is by measuring the flight time $t_{r e f}$ of a known reference mass $m_{\text {ref }}$. (2.8) can then be expressed as $m=m_{\text {ref }} / t_{\text {ref }}^{2} \cdot t_{f}^{2}$. Since the measurement conditions (electrode potentials, flight trajectories, etc.) vary constantly due to readjustments of experimental parameters, a reference measurement stays in general not valid. It can only be used as a coarse calibration of the data.

A better solution is to obtain the unknown values $k:=2 q U / l^{2}$ (or $k:=m_{\text {ref }} / t_{\text {ref }}^{2}$ ) from a fit of the measurement data. If one takes into account a certain time offset $t_{\text {off }}$ due detector response and signal propagation time, 2.8 reads as following:

$$
m=k\left(t_{f}-t_{\text {off }}\right)^{2} \text {. }
$$

If $k$ and $t_{\text {off }}$ are unknown, one needs at least 3 flight times $t_{f_{i}}$ corresponding to 3 different masses and their mass differences $\Delta m_{j} . k, t_{\text {off }}$ and $m_{\text {off }}$ can then be determined by minimizing

$$
k\left(t_{f_{i}}-t_{\text {off }}\right)^{2}-m_{i}-m_{\text {off }} .
$$

${ }^{14}$ LeCroy LC534AM 
One can choose $m_{1}=1, m_{2}=m_{1}+\Delta m_{1}$ and $m_{3}=m_{2}+\Delta m_{2}$ as start conditions. (2.10) becomes then minimal for the actual masses $m_{i}$.

\subsubsection{Photoelectron Spectra and Calibration}

The kinetic energy $E$ (in $\mathrm{eV}$ ) of photoelectrons can be calculated from the flight time $t$ for a drift length $l$ from the expulsion origin to detector plane:

$$
E(t)=\frac{m_{e}}{2 e}\left(\frac{l}{t}\right)^{2},
$$

whereas $m_{e}$ is the electron mass and $e$ the electron charge. Since the area under intensity curve $I(t)$ corresponds to the electron count, it must be kept constant, when transforming flight time to kinetic energy $t \rightarrow E: I(t)=I(E) d E / d t$. This means the Intensity $I$ must be scaled by:

$$
I(E(t))=I(t) \frac{t^{3}}{2 \frac{m_{e} l^{2}}{2 e}} .
$$

The binding energy spectrum is obtained by a further linear transformation of the energy scale (2.11):

$$
E_{b i n}(t)=h \nu-E(t),
$$

whereby $h \nu$ indicates the photon energy of the detachment laser.

Eq. (2.11) describes the kinetic energy in the ideal case without external interference. In a real experiment there are several factors which influence the measurement of the kinetic energy: The detector signal has a certain propagation time, which results in a time offset of the flight time $t+t_{\text {off }}$. The actual drift length is not known very accurately and additionally contains the short acceleration region to the electron multiplier. It is therefore better expressed by an effective length $l+\Delta l$. The kinetic energy of the photoelectrons can be altered by surface charge effects of the flight tube, which introduce an energy offset $E_{\text {off }}$. Allowing for all these effects (2.11) has to be changed to:

$$
E(t)=\frac{m_{e}}{2 e}\left(\frac{l+\Delta l}{t+t_{\text {off }}}\right)^{2}-E_{\text {off }} .
$$

Assorting all constants to $q:=m_{e} l^{2} /(2 e)$ and using $l_{s}:=(l /(l+\Delta l))^{2},(2.14)$ can be expressed as:

$$
E(t)=\frac{q}{l_{s}\left(t+t_{\text {off }}\right)^{2}}-E_{\text {off }}
$$

The kinetic energy is calibrated by means of spectra of the platinum anion $\left(\mathrm{Pt}^{-}\right)$. The energy levels of $\mathrm{Pt}^{-}$are tabulated[47] relative to its electron affinity of $2.1251 \mathrm{eV}[8]$. Table 2.1 lists the lowest binding energies up to $6 \mathrm{eV}$ relevant to visible and UV photoelectron spectroscopy. 


\begin{tabular}{ll||ll} 
level & $E_{\text {bin }}(\mathrm{eV})$ & level & $E_{\text {bin }}(\mathrm{eV})$ \\
\hline $5 d^{9} 6 s$ & 2.1251 & $5 d^{9} 6 s$ & 3.79843 \\
& 2.2213 & $5 d^{8} 6 s^{2}$ & 4.04708 \\
$5 d^{8} 6 s^{2}$ & 2.22723 & $5 d^{8} 6 s^{2}$ & 4.42705 \\
$5 d^{10}$ & $(2.88636)$ & $5 d^{8} 6 s^{2}$ & 4.84867 \\
$5 d^{8} 6 s^{2}$ & 2.93937 & $5 d^{8} 6 s^{2}$ & 5.42787 \\
$5 d^{8} 6 s^{2}$ & 3.37942 & $5 d^{8} 6 s\left({ }^{4} \mathrm{~F}\right) 6 p$ & 5.86409 \\
$5 d^{9} 6 s$ & $(3.38131)$ & $5 d^{9} 6 p$ & 6.16946
\end{tabular}

Table 2.1.: $\mathrm{Pt}^{-}$binding energy levels [8, 47]. Values in parenthesis are not used for fitting.

The values for $l_{s}, t_{\text {off }}$ and $E_{\text {off }}$ can be obtained by fitting a Gaussian function

$$
g_{j}\left(E, E_{j}, A, \sigma\right)=A \exp \left(-\frac{\left(E-E_{j}\right)^{2}}{2 \sigma^{2}}\right) .
$$

for each binding energy level $E_{j}$ to the peaks of the spectrum.

Since the measured value is the flight time $t$ and the energy transformation of the spectra requires scaling of the Intensity, it is preferable to fit the time of flight spectrum rather then the energy spectrum. The calibration of the flight time can be derived from 2.15):

$$
t_{c a l}=\frac{1}{\sqrt{l_{s}\left(1 / t^{2}-E_{o f f} / q\right)}}-t_{\text {off }}
$$

The Gaussian function (2.16) transformed to fit the flight time peaks reads:

$$
\begin{aligned}
& g_{j}\left(t, E_{j}, t_{\text {off }}, E_{\text {off }}, l_{s}, A, \sigma\right)= \\
& \frac{A 2 q}{t^{3}} \exp \left(-\frac{\left(q\left(\frac{1}{\sqrt{l_{s}\left(1 / t^{2}-E_{\text {off }} / q\right)}}-t_{\text {off }}\right)^{-2}-E_{j}\right)^{2}}{2 \sigma^{2}}\right) .
\end{aligned}
$$

Fig. 2.10 shows a fit of multiple Gaussian functions $G=\sum g_{j}$ to the flight time spectrum of $\mathrm{Pt}^{-}$. In this particular example the fit $G$ (blue line) coincides so well with the experimental data (black line) that it covers it almost completely.

Platinum anions are produced in a pulsed discharge source and are ejected directly into the first acceleration stage of the time-of-flight spectrometer. The source consists of a pulsed valve, which produces high density He gas pulses. When a gas pulse reaches the platinum wire mounted into the nozzle of the source, a high voltage pulse is applied to the wire, starting a plasma discharge. Platinum ions form in the plasma and are carried out by the gas stream. 

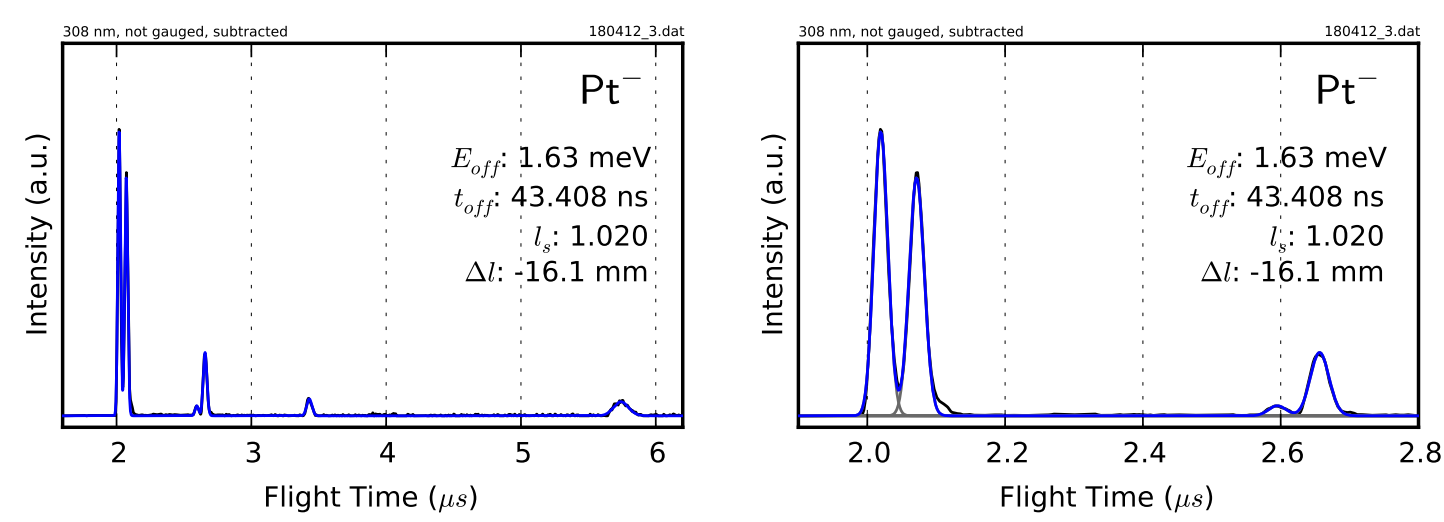

Figure 2.10.: Fit (blue) to the flight time spectrum of Pt anions (black). The right panel shows an enlargement of the first four peaks.

The propagation time $t_{\text {off }}$ of the detector signal of the photoelectron spectrometer can be estimated by measuring the signal from scattered laser photons, produced e.g by placing a metal wire into the interaction zone. The photodiode signal from non-scattered photons defines the emission time of the detector signal. Fig. 2.11 shows such a measurement. The pulse reaches its maximum at about $45 \mathrm{~ns}$, which demonstrates that the time offsets $t_{\text {off }}$ resulting from fits (mostly $40 \mathrm{~ns}$ to $50 \mathrm{~ns}$ ) are quite reasonable.

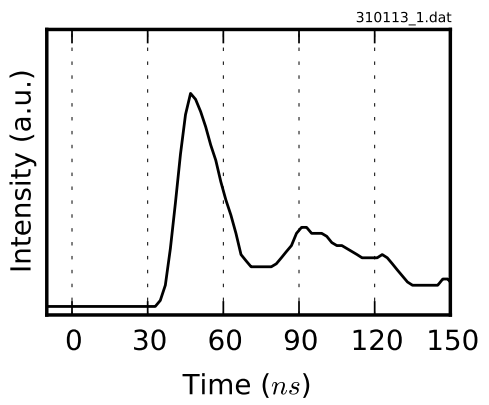

Figure 2.11.: Measurement of the delay caused by the propagation time of the detector signal.

\section{Discussion}

The measurements of the calibration spectra and the spectra, to which the calibration is later applied, take place at different times. For practical reasons the time difference amounts to days or even months ${ }^{15}$. This approach is of course only valid,

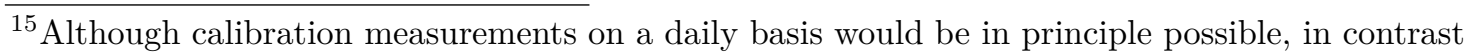
to the mass spectroscopy. 
if the calibration parameter $\left(l_{s}, t_{\text {off }}, E_{\text {off }}\right)$ stay stable for such time intervals.

In fig. 2.12 the fit parameters originating from measurements taken over several years are compared by plotting eq. (2.17). The most influential parameter is the energy offset $E_{\text {off }}$ (listed in $\mathrm{meV}$ ). It can be used to group the data by time intervals, in which $E_{\text {off }}$ resulted in similar values. The energy offset is caused by surface charges of the inner drift tube of the spectrometer. Any change in the surface conditions can therefore alter the surface charge and consequently the energy offset. This means that large shifts in $E_{\text {off }}$ are to be expected after alterations of spectrometer, like venting of the vacuum chamber or replacement of parts, occurred.

Even within a time interval with an approximately fixed $E_{\text {off }}$, the measurements show still small variation of the fit parameters. Part of this may be caused by real fluctuations. For example the flight length can vary slightly $(\Delta l)$ by a few $\mathrm{mm}$ depending on the crossing point of the cluster beam and laser. The mayor part, however, must probably be attributed to statistical nature of the experimental spectra: Small variations in the peak shapes causes the fit to converge with slightly different fit parameters.

Fig. 2.12 demonstrates the influence of the fit parameters on the energy representation of the spectra. It underlines once more that the energy offset has to be considered in any case for a valid calibration. The small variation caused by other factors become only significant for a high resolution in the high binding energy region of the spectra.

As a general conclusion follows that for most cases the calibration parameters stay valid in times without modifications of the photoelectron spectrometer and its vacuum chamber. This is especially true for measurements containing their relevant data in the lower $85 \%$ of the binding energy range, e.g. spectra of metal or semiconductor clusters.

For higher accuracy in the high binding energy region of the spectrum one could use the mean value of the fit parameters. Another approach to reduce the variation of the fit parameters due to convergence differences, could be the use of a different element for the reference measurement. Iridium for example has a lower electron affinity $(1.56 \mathrm{eV})$ than platinum $(2.12 \mathrm{eV})$ and many, quite evenly spread energy levels (cf. tab2.2). In fig. 2.13 the peak distribution of both elements in the binding energy range from $0 \mathrm{eV}$ to $4.02 \mathrm{eV}$ is plotted for comparison.

The iridium anions are produced analogously to $\mathrm{Pt}^{-}$in the pulsed discharge source by replacing the platinum wire with an iridium wire.

\subsubsection{Issues Recording Photoelectron Spectra of Water Cluster Anions}

The measurements of photoelectron spectra of water cluster anions revealed a series of experimental issues, which are either immanent to the processing of water clusters 


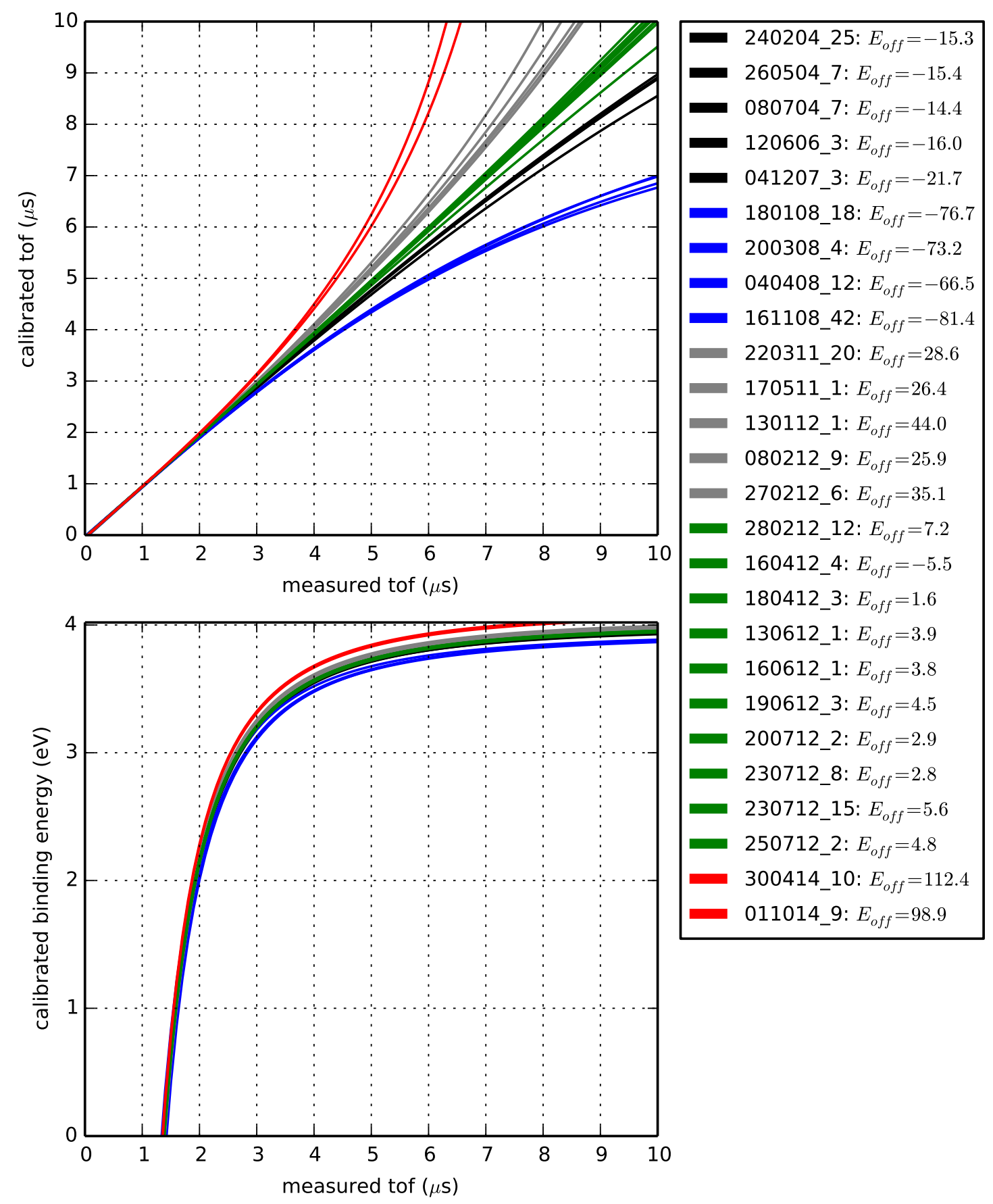

Figure 2.12.: Upper panel: Calibration of the time-of-flight (tof) according to eq. 2.17) with fit parameters $\left(l_{s}, t_{\text {off }}, E_{\text {off }}\right)$ from $\mathrm{Pt}^{-}$measurements gathered over several years. The colors indicate time intervals, for which the fits resulted in similar energy offsets $E_{\text {off }}$ (in meV).

Lower panel: Same data as above, applied to calibrate the binding energy according to (2.13) and 2.15) for $4.02 \mathrm{eV}$ photons. 

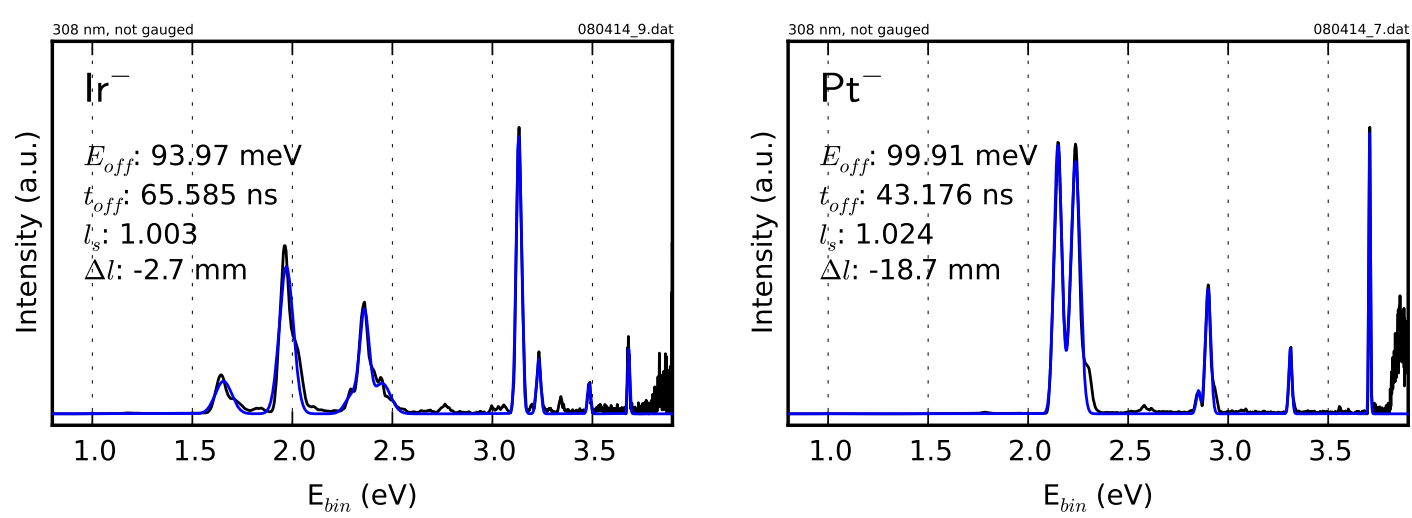

Figure 2.13.: Comparison of the PES peak distribution of Ir and Pt anions for the same binding energy range. The fits (blue lines) were made to the respective time-of-flight spectrum.

\begin{tabular}{ll||ll} 
level & $E_{\text {bin }}(\mathrm{eV})$ & level & $E_{\text {bin }}(\mathrm{eV})$ \\
\hline $5 d^{7} 6 s^{2}$ & 1,56436 & $5 d^{8}\left({ }^{3} P\right) 6 s$ & $(3,170162)$ \\
$5 d^{8}\left({ }^{3} F\right) 6 s$ & 1,915853 & $5 d^{8}\left({ }^{3} F\right) 6 s$ & 3,187053 \\
$5 d^{7} 6 s^{2}$ & $(2,070084)$ & $5 d^{7} 6 s^{2}$ & 3,292675 \\
& 2,281561 & $5 d^{7} 6 s^{2}$ & 3,560917 \\
& 2,348425 & $5 d^{8}\left({ }^{3} P\right) 6 s$ & $(3,618202)$ \\
$5 d^{8}\left({ }^{3} F\right) 6 s$ & 2,445467 & & $(3,632565)$ \\
& 2,789019 & $5 d^{7} 6 s^{2}$ & 3,768705 \\
$5 d^{8}\left({ }^{3} P\right) 6 s$ & $(2,875949)$ & $5 d^{7} 6 s^{2}$ & $(3,8639)$ \\
$5 d^{8}\left({ }^{3} F\right) 6 s$ & $(3,031228)$ & $5 d^{8}\left({ }^{1} D\right) 6 s$ & $(3,927576)$ \\
$5 d^{8}\left({ }^{3} F\right) 6 s$ & $(3,079257)$ & $5 d^{7} 6 s^{2}$ & 3,993613 \\
$5 d^{8}\left({ }^{3} P\right) 6 s$ & $(3,114867)$ & &
\end{tabular}

Table 2.2.: $\mathrm{Ir}^{-}$binding energy levels [8, 48]. Values in parenthesis are not used for fitting.

or are particularly visible in their spectra. In the following sections we discuss the (possible) sources of the interferences, their impact on the spectra and the counter measures, which enables us to evaluate the affected spectra nevertheless.

\section{“Echo" Peaks in Early Measurements}

The initial photoelectron spectra of water cluster anions exhibit distinct additional peaks on the high binding energy slope of the main peak. The peaks are equidistantly spaced in respect to the flight time, whereas the main peak marks the beginning of the peak series. From both laser wavelengths used at that time, only the spectra recorded at $308 \mathrm{~nm}$ are affected. The spectra at $590 \mathrm{~nm}$ do not show 
additional peaks (fig. 2.14, right panel). The lower laser intensity of the dye laser used for the $590 \mathrm{~nm}$ photons, probably produces a low enough photoelectron signal to prevent ion feedback.
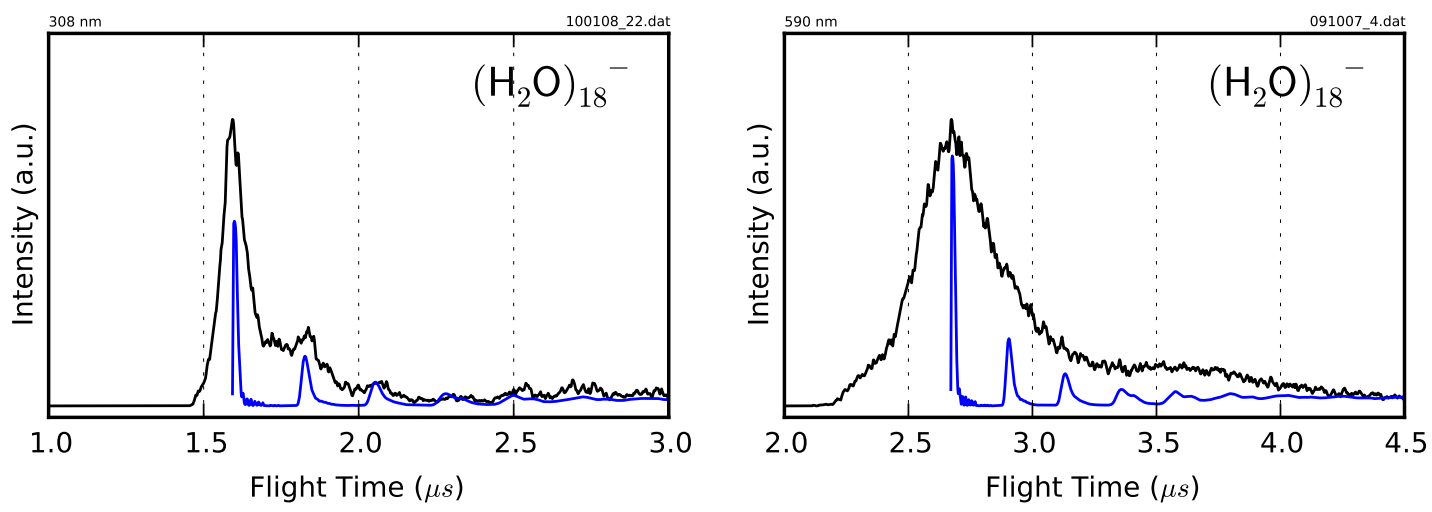

Figure 2.14.: Photoelectron spectra of $\left(\mathrm{H}_{2} \mathrm{O}\right)_{18}{ }^{-}$(black lines) demonstrating the presence of additional peaks when using $308 \mathrm{~nm}$ (left panel) and the absence for $590 \mathrm{~nm}$ (right panel) respectively. The blue lines show the spectrum of the ion feedback test measurement (cf. fig. 2.15)

The cause of the additional peaks could be identified as ion feedback in the single channel electron multiplier of the photoelectron spectrometer (cf. section 2.6). As verification the detector signal was recorded, while flooding the interaction region of the spectrometer with UV-photons from a glow-discharge lamp. Figure 2.15 shows the appearance of feedback peaks in dependency of the multiplier voltages $V_{a c c}$ and $V_{a m p}$. Especially a higher bias $V_{a m p}$ causes more "echo" peaks. The time between the peaks ranges between $220 \mathrm{~ns}$ to $250 \mathrm{~ns}$. By comparing the feedback peak spectrum to the cluster spectra (blue lines in figure 2.14), the peaks originating from ion feedback can be explicitly associated.
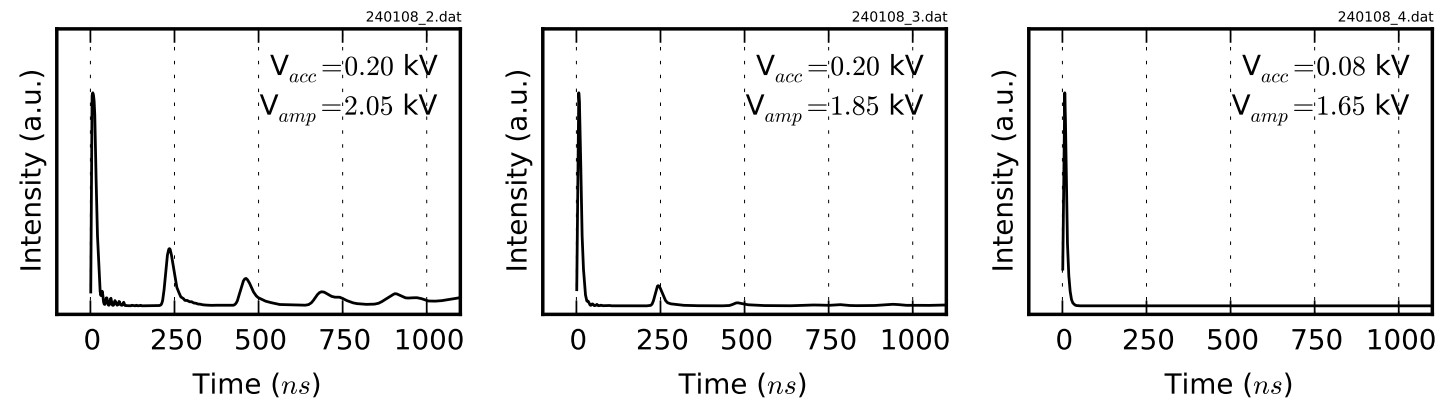

Figure 2.15.: The number of "echo" peaks depends on the electron multiplier voltages.

After having identified the cause of the "echo" peaks, the multiplier voltages 
for all further measurements were adapted to produce the maximum gain without showing artificial peaks.

\section{Detector artifacts}

An additional feature starting at about $3.3 \mathrm{eV}$ is visible in varying degrees in all spectra. It has been found that it is visible even in background spectra ${ }^{16}$ (fig. 2.16). The origin of this contribution could not be identified completely. More thorough investigations revealed that although the shape (in particular the onset at $3.3 \mathrm{eV}$ ) remained always the same, the intensity increased with the uptime of the channeltron electron multiplier. This may indicate that this particular background signal is produced within the electron multiplier, possibly by some sort of ion feedback.
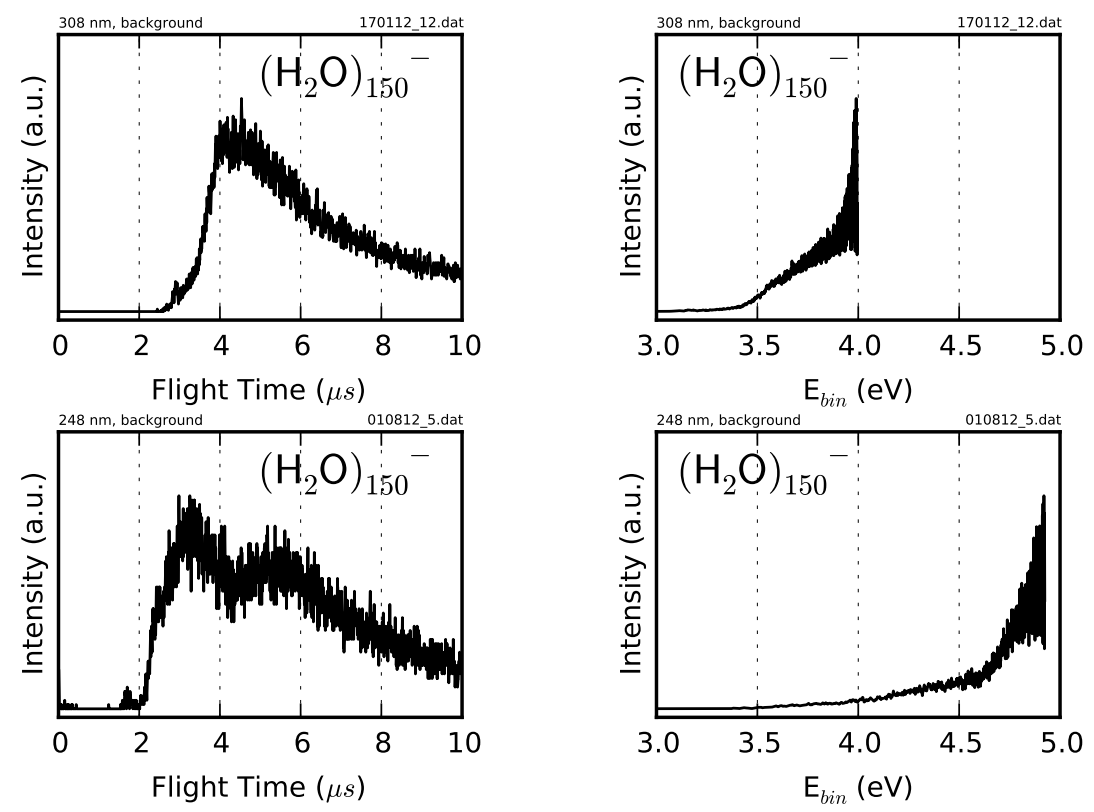

Figure 2.16.: Background intensity plotted versus the flight time and binding energy, recorded at $308 \mathrm{~nm}$ (upper row) and $248.4 \mathrm{~nm}$ (lower row) respectively. The intensities are scaled for better reading and are not comparable.

In order to eliminate the background contributions to the spectra, background spectra have been recorded directly after a cluster measurement. However, due to the non-constant intensity, the spectra gained by subtracting the background were not satisfactory, because either too much or not enough were subtracted. Therefore

${ }^{16}$ Spectra recorded under identical experimental conditions but without clusters in the interaction zone. 
a side by side measurement of the cluster and background spectrum was conducted, allowing to record the background during the time of the cluster measurement.

\section{Decreasing Signal Intensity with Increasing Cluster Size}

Another issue with the spectra of the sizes 250 and larger, is a decreasing signal to noise ratio. For those sizes the photoelectron count per laser shot stemming from the cluster electrons gets lower the bigger the clusters are. The main reason for this lies in the decreasing number of clusters in laser interaction zone. A qualitative explanation for the decreasing ion beam signal can be derived, if one looks at the mass distribution stored in the rf ion trap: storage conditions for larger clusters masses are suitable for a broader size range. Since the trap has only a certain storage capacity (and is presumably filled to its limit), the percentage of a single cluster size is lower under such conditions. However, there might be other causes, like a reduced absorption cross section for larger clusters, which were not investigated further.

The reduced intensity of the large water cluster spectra accentuates minor perturbation, which are not particular prominent otherwise in the high intensity spectra of smaller cluster sizes. Especially during the measurements of clusters with more than 300 water molecules, deteriorated the cluster ion signal the longer the experiment was running. Consequently only the first few measurements of a measurement cycle had adequate intensities for evaluation. One possible explanation for this uptime deterioration of the signal, is the formation of an ice film on the entrance electrode of the trap. It has been found in earlier experiments that an electrically isolating coating can reduce the storing efficiency of the trap. 


\section{Theoretical Background}

One of the recurring findings of cluster experiments is the strong size dependency of the observables. The properties of small and medium sized clusters show in general a highly discontinuous evolution with the cluster size and develop into a more continuous progression only for very large sizes. The cause for this "every atom counts"-behavior lies in the cluster being a finite, nano-scaled system, which places its governing physics firmly in the quantum mechanical regime. An accurate description of the properties of the cluster must therefore include such effects. On the other hand clusters are huge systems of many interacting particles, which makes a complete quantum mechanical treatment impossible. A theoretical model accounting for all those effects must ultimately solve the Schrödinger equation. Due to the size and complexity of the cluster as quantum mechanical system, this is only possible by making a considerable number of approximating assumptions, which greatly simplify the problem. In the end, the crucial step is therefore to find a compromise between a reasonable approximation of the Schrödinger equation and the computational costs of its solution.

A more elaborated treatment of this subject is beyond the scope of this work. In the light of the strongly varying results of theoretical studies, which we will encounter in section 4.3.2, it is instructive to have a short look at the limiting factors. Almost all theoretical calculations treat only the excess electron and in few cases the valence electrons of the water molecules fully quantum mechanically, whereas the water molecules are handled classically or semi-classically. Roughly expressed lie the details and differences of the various approaches in the electron-water and water-water potentials, as well as in the treatment of the polarization interaction. The start configuration and temperature of the cluster, the time allowed to finds its equilibrium structure and the time of excess electron attachment also have a strong impact on the results (cf. [5, 6, 32, 36, 73-75]).

Besides such for accuracy striving approaches, it is, however, often possible to find a simple scaling law [7], which describes the evolution of a cluster property with its size $n$ more generally:

$$
\xi_{n}=\xi_{\infty}+\alpha n^{-\beta}
$$

where $\xi_{n}$ and $\xi_{\infty}$ are the value of the property for size $n$ and the bulk respectively. $\alpha$ is a constant and depends on the cluster material (and the property in question). $\beta$ is a number $\geq 0$. Such scaling laws are of course unable reproduce the size dependent local details, but can give a general trend, which becomes an increasingly 
better approximation with increasing cluster size.

In many cases the scaling is proportional to either the volume or the radius of the clusters. If one defines the cluster volume as the sum of the volumes of its base units, i.e. the atoms or molecules of which it consists,

$$
V_{\text {cluster }}=\frac{4}{3} \pi R^{3}:=n \frac{4}{3} \pi r_{b u}^{3},
$$

then the volume is proportional to the number of base units $n$ and the radius is proportional to $n^{1 / 3}$ respectively. That means $\beta=1$ or $\beta=1 / 3$ corresponds to the inverse of the volume and the inverse radius respectively.

In an early publication about the size dependence of the energetics of excess electron in water cluster anions [5], Barnett et al. have demonstrated that the size evolution of the adiabatic and vertical detachment energies of the interior localized excess electron of large clusters can be approximated by the dielectric sphere model. The dielectric sphere model states that the adiabatic binding energy of an excess electron can be obtained from the total energy of an electron in a dielectric sphere, given a spherical symmetry of the electron charge distribution, uniform dielectric properties of the cluster, and a cluster radius considerably larger than the electrons radius of gyration. The vertical binding energy is calculated from the adiabatic binding energy by adding the medium reorganization energy, which is the energy required to change the dielectric sphere in terms that the electronic orientation component of the polarization field adapts to a neutral dielectric sphere, while the molecular orientation component remains the value of negatively charged system [5].

The expression for the experimentally relevant vertical detachment energy (VDE) shows a linear relation with the inverse cluster radius:

$$
\operatorname{VDE}(n)=\mathrm{VDE}_{\infty}+A n^{-\frac{1}{3}}
$$

The intercept, $\mathrm{VDE}_{\infty}$, corresponds to vertical detachment energy of the bulk. The slope $A$ depends on the relative permittivity at frequency $\nu, \epsilon_{r, \nu}$, and the relative static permittivity $\epsilon_{r, s}$, which is frequency independent $\nu=0$ :

$$
A=\frac{e^{2}}{2 r_{b u}}\left(1+\frac{1}{\epsilon_{r, \nu}}-\frac{2}{\epsilon_{r, s}}\right),
$$

where $e$ is the charge of the electron and $r_{b u}$ the mean radius the water molecule.

The vertical detachment energy (VDE) is one of the key observables gained from photoelectron spectroscopy of water cluster anions. The binding energy with the highest abundance of photoelectrons in the spectrum is interpreted as the VDE. The actual spectral line shape is offhand not easily determinable. But since the maximum defines the experimental VDE, the general peak shape should be discussed at least qualitatively. 
The left side of fig. 3.1 shows a schematic potential energy diagram of the excess electron in the anionic solvation coordinate. Two electronic transitions are indicated, one representing the excitation of a p-state, relevant for absorption or pump-probe measurements, and the other the photoejection process of the photoelectron spectroscopy. The black arrows symbolize transitions from the most likely ground state configuration, which would result in narrow lines in the spectrum. For a given cluster configuration there are also vibrational transitions according to the Franck-Condon principle resulting in a asymmetric broadening of the line towards higher binding energy photoelectrons. This is symbolically depicted by the reddish arrows and the red spectra in the center of fig. 3.1 respectively.

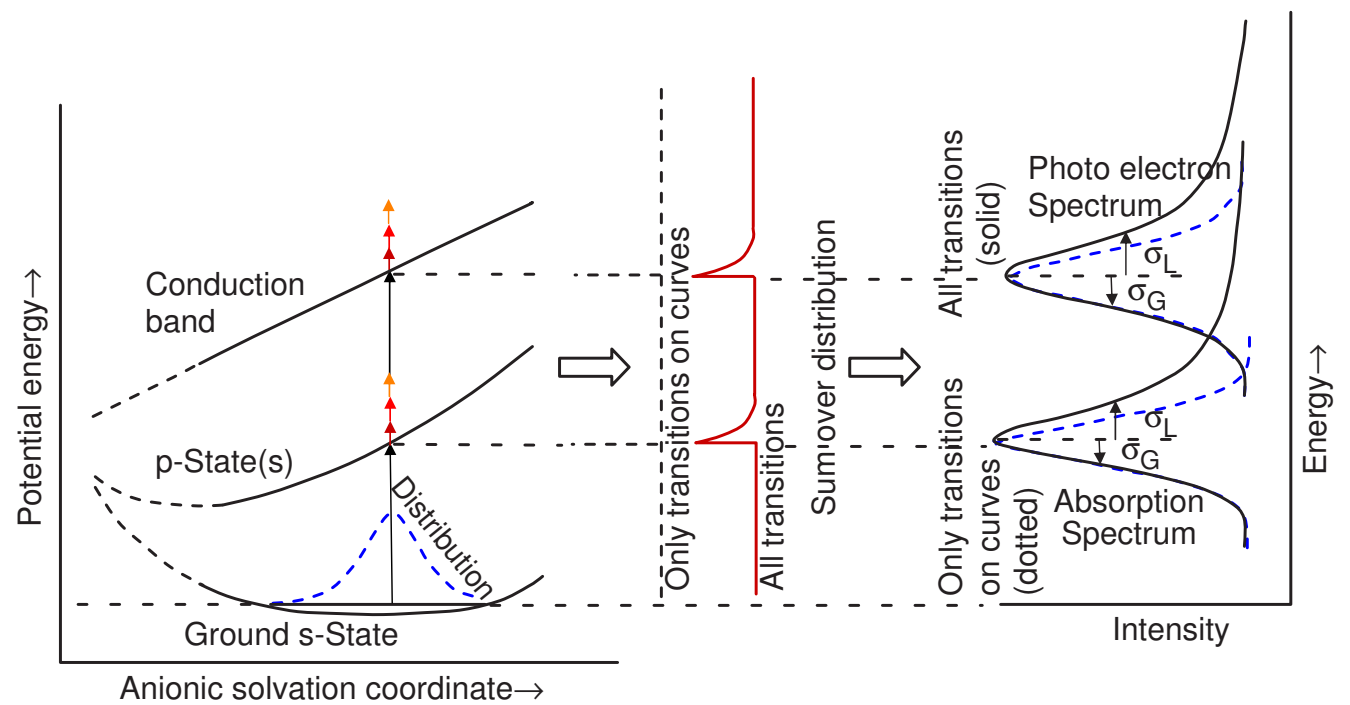

Figure 3.1.: Left: Potential energy diagram of a water cluster anion. Middle: Schematic spectral contribution by a single structure. Right: sum spectrum resulting from all structures and their individual contributions (black lines) or just the structural distribution without vibrational broadening. Based on [18].

The structures of clusters in an experimental beam vary to a certain degree, since especially larger clusters will have a great number of energetically close lying isomers. The abundance of the different configurations follows also a distribution, which is indicated by the blue peak in the potential energy diagram. The contributions originating from a single cluster configuration must be therefore summed up and weighted by this configuration distribution. The resulting spectra are broad, asymmetric peaks, shown as black lines in the right part of fig. 3.1. The high binding energy tail of the total spectrum originates in this picture from the vibrational contributions of each cluster configuration. If the width of these contributions (the red ones) is narrow in comparison to the width of configuration distribution (the 
blue one), then the low binding energy side of the composite spectrum is mainly determined by the structural configuration distribution, whereas the high binding energy side is strongly shaped by the nature of the single configuration contribution.

The discussed pictures assumes a single isomer or, more specific, a isomer class, which consists of all structural variations resulting in similar VDEs. Theoretical models predict, however, different isomers based on the excess electrons localization with distinctly different VDEs. Besides the surface and the interior or cavity localized ones of the early models [5] there are now predictions for other types like surface cavities[36], surface diffuse[6], and non-cavity interior [72] states. Occurrences of such isomers in an experimental cluster beam would add additional features to the spectra. In the simplest case one can assume for each a similarly compounded isomer class spectrum as described above. In the case of well separated VDEs this will result in additional peaks, whereas for closely lying VDEs each isomer class will contribute to a common peak according to its abundance in the beam. Especially the second case with two or more isomer classes, can make the deconvolution of experimental spectra difficult or even impossible due to the intrinsic broad contribution of each class. 


\section{Water Cluster Anions}

\subsection{Experimental Data}

Water cluster anions of a wide size range, from a few water molecules up to over thousand, have been produced, size selected and investigated by photoelectron spectroscopy. In the size range between 16 to 105 water molecules each size has been measured. For medium sized clusters between 105 and 150 a size step of about 10 has been chosen and for large clusters the step size amounts to 50 or 100 water molecules respectively. The majority of spectra were measured with a trap temperature between $10 \mathrm{~K}$ to $100 \mathrm{~K}$. For certain sizes, mainly around 21 and 48 to 51, measurements at higher trap temperatures up to room temperature have been conducted. The photon energy used for photoelectron detachment was $4.02 \mathrm{eV}(308 \mathrm{~nm})$. To verify the independence of the PES from the photon energy, photoelectron spectra of few cluster sizes were additionally recorded at $4.99 \mathrm{eV}$ $(248.4 \mathrm{~nm})$ and $2.1 \mathrm{eV}(590 \mathrm{~nm})$. The flight time and energy scale respectively of all spectra were calibrated with $\mathrm{Pt}^{-}$reference spectra (cf. section 2.8.2) recorded in between from time to time.

It should be noted that the $590 \mathrm{~nm}$ spectra were calibrated using calibration parameters from $308 \mathrm{~nm}$ reference spectra. Although the reference spectra are usually recorded at the same wavelength as the normal spectra, the resulting calibration parameter are solely dependent on the spectrometer and not on the utilized wavelength (cf. Discussion in section 2.8.2.

\subsubsection{Mass Spectra}

The mass spectrum of water cluster anions shows in general no structure or modulation other than the one caused by the measurement apparatus itself. Clusters containing less than $15 \mathrm{H}_{2} \mathrm{O}$ molecules have a very low abundances or are none existent in the spectrum (fig. 4.1, left). The shoulders of the water peaks originate on the left slope (lower mass) from clusters with a single $\mathrm{HO}$ attached and on the right slope (higher mass) from clusters with a different isotopic distribution. This is due to the isotopic composition of water [25] with ${ }^{16} \mathrm{O}$ present in $99.76 \%,{ }^{17} \mathrm{O}$ in $0.04 \%$, and ${ }^{18} \mathrm{O}$ in $0.2 \%$ of water molecules. The small peaks well separated from the main peaks have a mass difference of $4 \mathrm{u}$ to their nearest water peaks. They are probably clusters with $4 \mathrm{HO}$ attached. 
For larger clusters the number of isotopic peaks per cluster size increases while simultaneously the peaks broaden due to higher masses. Finally only the envelope remains visible (fig. 4.1, right). The right panel of fig. 4.1 also includes simulated mass spectra at various resolutions to demonstrate the underlying isotopic peak distribution (gray line, at $R=m / \Delta m=20000$ ) and its envelope (blue line, at $R=2000)$ respectively.
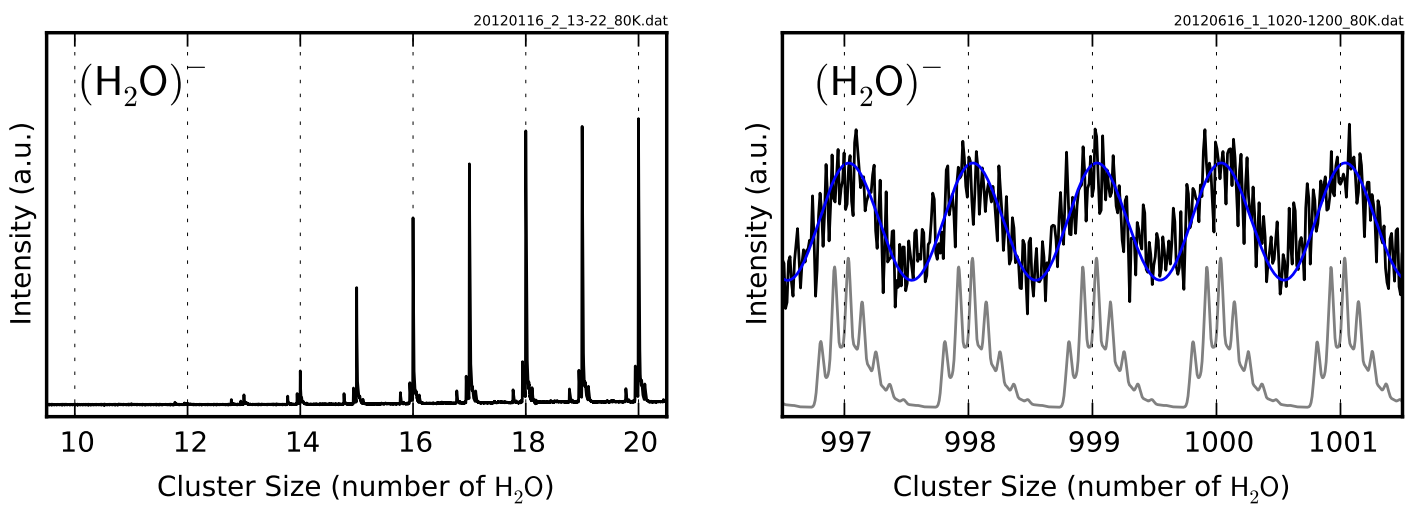

Figure 4.1.: Mass spectra (black lines) of small and large water cluster anions. The blue and gray lines are simulated mass spectra at a resolution of $m / \Delta m=2000$ and 20000 respectively.

In the size range of 50 to 60 water molecules the mass spectra show a modulation of abundances, which becomes particularly evident at temperatures between $100 \mathrm{~K}$ and $140 \mathrm{~K}$ (fig. 4.2): The cluster sizes 50, 52, 54, and 56 (magic sizes) show a significantly higher intensity than their neighboring peaks. At lower (below $80 \mathrm{~K}$ ) and
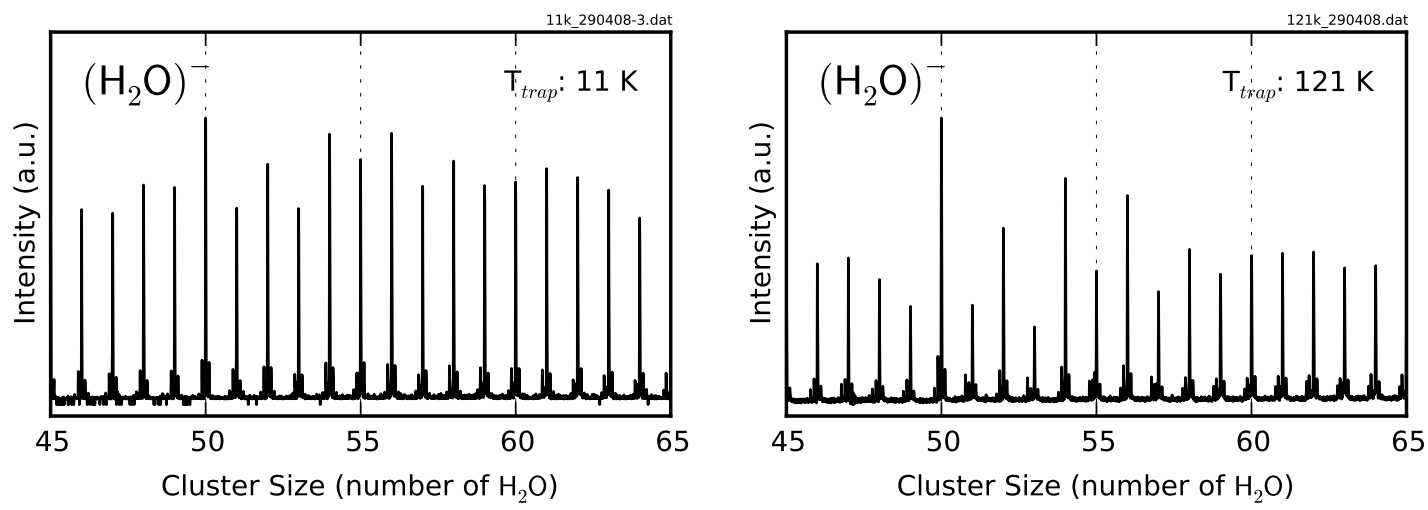

Figure 4.2.: Mass spectra of water cluster anions displaying the unusual modulation of abundances (magic sizes) in the size range of 50 to 60 water molecules.

higher temperatures (above $200 \mathrm{~K}$ ) the differences in abundance are considerably 
smaller, however, even at such temperatures a distinction is still possible (cf. left panel of fig. 4.2). That the magic numbers are still visible at very low trap temperatures suggests that the clusters get heated up between source and trap, since at such low trap temperatures, the trap conserves the abundance distribution entering the trap.

\subsubsection{Photoelectron Spectra}

In general the photoelectron spectra (PES) of all cluster sizes show a broad, featureless and slightly asymmetric peak, which exhibits a steeper incline on the low binding energy side and levels out more slowly on the high energy side. As a common trend one finds that the peak maximum shifts to higher binding energies and the peak width broadens with increasing cluster size (figure 4.3).
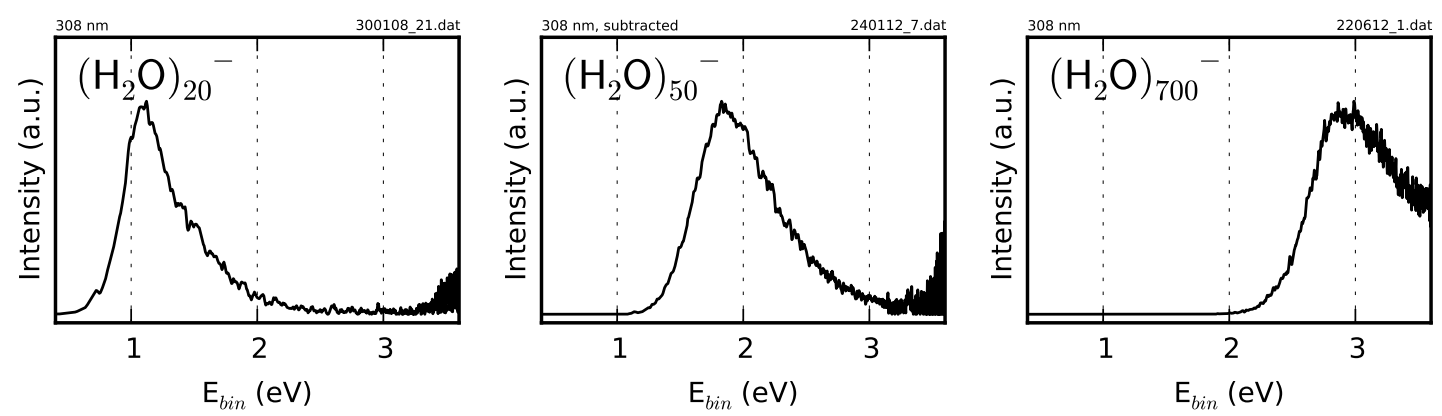

Figure 4.3.: Example photoelectron spectra of small, medium and large water cluster anions.

Some sizes deviate from the usual spectral shape by showing additional shoulders or even peaks on the slopes of the main peak. In few cases is the intensity of the additional features highly temperature dependent.
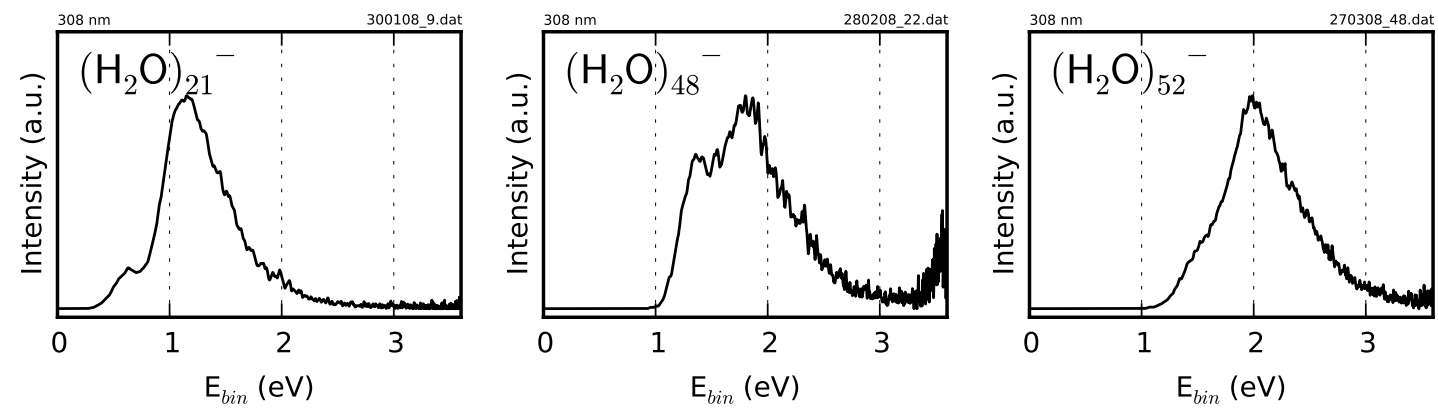

Figure 4.4.: Example photoelectron spectra of cluster sizes deviating from the general single peak trend. 


\subsection{Peak Analysis}

\subsubsection{Method}

For a more quantitative analysis of the spectra, a method to locate the maximum of the main peak and possible contribution by further peaks has to be found. The common approach is to fit one or more Gaussian-Lorentzian (GL) functions to the binding energy spectrum. By doing so the low binding energy part of the spectrum to the intensity maximum at $E_{0}$ is described by a Gaussian peak shape with a width $\sqrt{2 \ln (2)} \sigma_{G}\left(\sigma_{G}\right.$ is the standard deviation of the Gaussian part) and the high binding energy part by a Lorentzian with width $\sigma_{L}$ :

$$
I(E)= \begin{cases}A \exp \left(-\frac{\left(E-E_{0}\right)^{2}}{2 \sigma_{G}^{2}}\right) & \text { if } E \leq E_{0} \\ \frac{A}{\frac{\left(E-E_{0}\right)^{2}}{\sigma_{L}^{2}}+1} & \text { if } E>E_{0} .\end{cases}
$$

The use of GL functions is motivated by the fact that they empirically reproduce the line shape of photoelectron spectra of water cluster anions and absorption spectra of the solvated electron in bulk water [18].

The peak analysis of our experimental spectra has shown that the location of the maximum is very sensitive to the progression of the high binding energy tail. There are two factors which might influence the fit: the energy-dependent scaling of the noise amplitude due to the transformation from the time-of-flight to the energy scale and the detector artifact mentioned in section 2.8.3. For both cases it is preferable to fit against the time-of-flight spectrum. The noise amplitude is constant in time space and the artifacts are clearly separated from the original spectrum and can be excluded from the fit. This is done by fitting the data only in a certain interval. Fig. 4.5 shows the PES of a $\left(\mathrm{H}_{2} \mathrm{O}\right)_{600}{ }^{-}$cluster against the binding energy and the time-of-flight respectively. The blue line represents the GL fit in time space (for both spectra). The solid part of the line indicates the data region used for the fit.

In order to conduct the fit in time space, the GL function (4.1) has to be transformed:

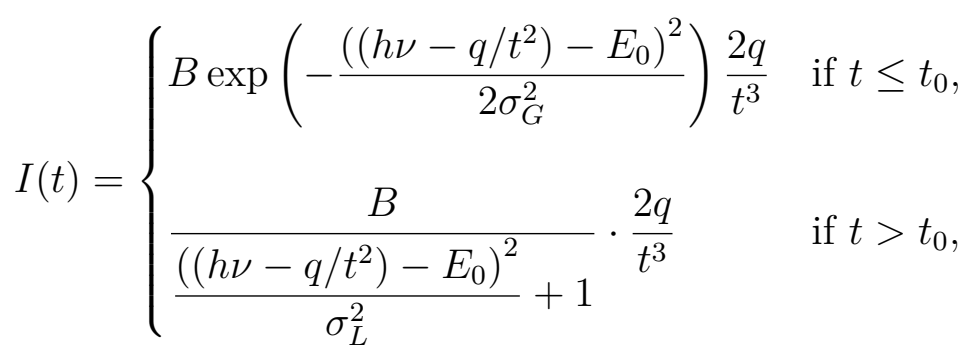



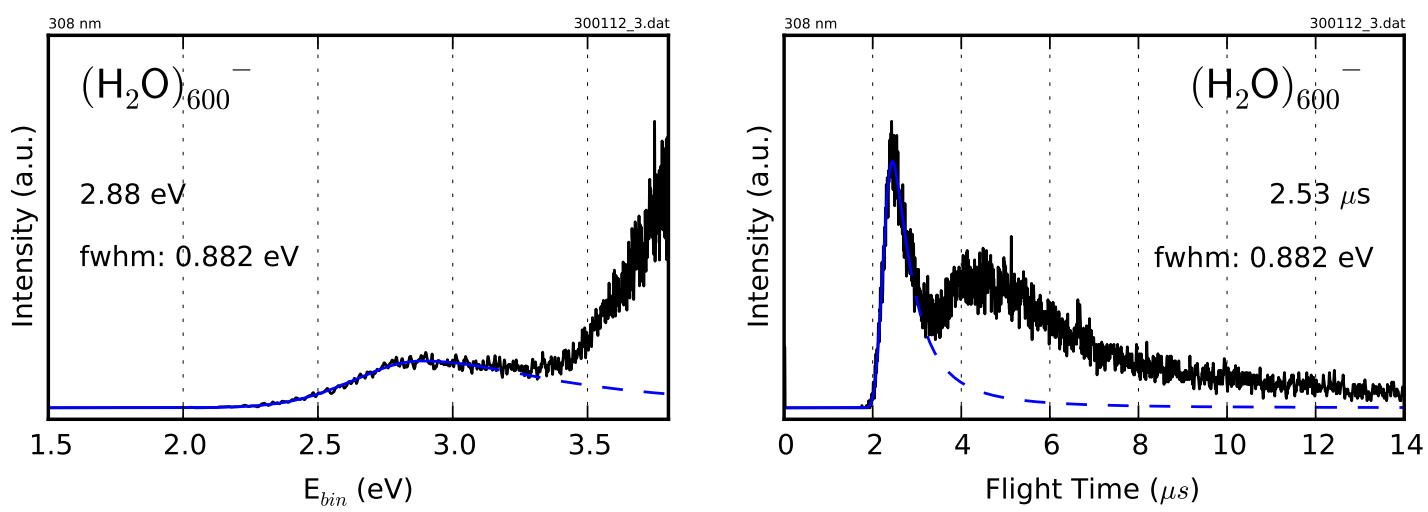

Figure 4.5.: Single GL fit (blue line) to the binding energy (left) and the time-offlight (right) photoelectron spectrum of $\left(\mathrm{H}_{2} \mathrm{O}\right)_{600}{ }^{-}$.

$$
q=\frac{m_{e} \cdot l_{p e s}^{2}}{2 e}, \quad E_{0}=h \nu-\frac{q}{t_{0}^{2}}, \quad B=A_{t} \frac{t_{0}}{2\left(h \nu-E_{0}\right)}=A_{t} \frac{t_{0}^{3}}{2 q} .
$$

The parameters gained from the fit are the rescaled amplitude $A_{t}$, the flight time $t_{0}$ corresponding to the energy at the intensity maximum, and the width parameter of GL function $\sigma_{G}$ and $\sigma_{L}$. The width of the peak at half the maximum intensity $(f w h m)$ is related to the parameters by:

$$
f w h m=\sqrt{2 \ln (2)} \sigma_{G}+\sigma_{L} .
$$

As will be discussed in detail in the following sections, some cluster sizes exhibit shoulders, a broadening of the main peak or even an additional peak. A fit with a single GL function therefore deviates substantially from the experimental spectrum. To get a better approximation of the data, such sizes were also fitted with multiple GL functions $I_{\Sigma}(t)=\sum I_{i}\left(t, A_{t, i}, t_{0, i}, \sigma_{G}, \sigma_{L}\right)$ with variable Amplitudes $A_{t, i}$ and peak positions $t_{0, i}$, but with identical width parameters $\sigma_{G}$ and $\sigma_{L}$.

\subsubsection{First Approximation: Single GL Fit}

For a detailed analysis of the experimental data we can start with the simplest approximation, that is by comparing it to single GL fit. In the following we will concentrate on few examples from a certain size range to demonstrate the evolution of spectral attributes in that range. A complete listing of all spectra can be found in sections A.1 to A.3 in the appendix.

\section{Sizes 15 to 27}

As we have already seen in the mass spectrum of small water clusters (sec. 4.1.1), an adequate intensity for recording photoelectron spectra starts with clusters consisting of $15 \mathrm{H}_{2} \mathrm{O}$ and larger. In the size range from 15 to 27 the agreement of the 
experimental spectra with a single GL function is essentially quite good. Only the high binding energy slope deviates very slightly. The deviation is better visible in the $590 \mathrm{~nm}$ measurements, which show a small shoulder (fig. 4.6, left).
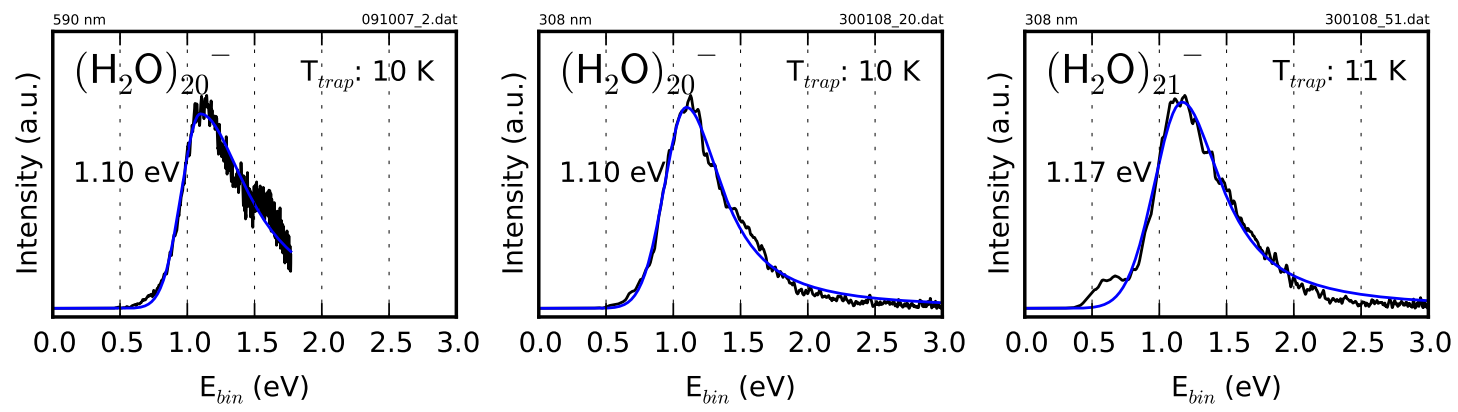

Figure 4.6.: Example photoelectron spectra from the size range $n=15$ to 27 .

The sizes 21, 24, 25, and 26 feature all a more or less distinct shoulder on the onset of the low binding energy slope of the main peak. It is only visible in spectra recorded at a temperature between $10 \mathrm{~K}$ and $100 \mathrm{~K}$ to $120 \mathrm{~K}$. For the $\left(\mathrm{H}_{2} \mathrm{O}\right)_{21}{ }^{-}$ cluster it becomes prominent enough to be seen as a discrete peak (fig. 4.6, right). Since the low binding energy slope is in general steeper than high binding energy slope, such shoulders can not be captured by a single GL function.

\section{Sizes 28 to 33}

The spectra of water cluster anions with 28 to 33 water molecules show an overall good agreement with a single GL fit. However, the deviation of the high binding energy slope, which was barely visible for the smaller clusters, becomes even more explicit in this size range (fig. 4.7). The peak width at half of the maximum intensity $(f w h m)$ also increases from about $0.6 \mathrm{eV}$ to $0.7 \mathrm{eV}$
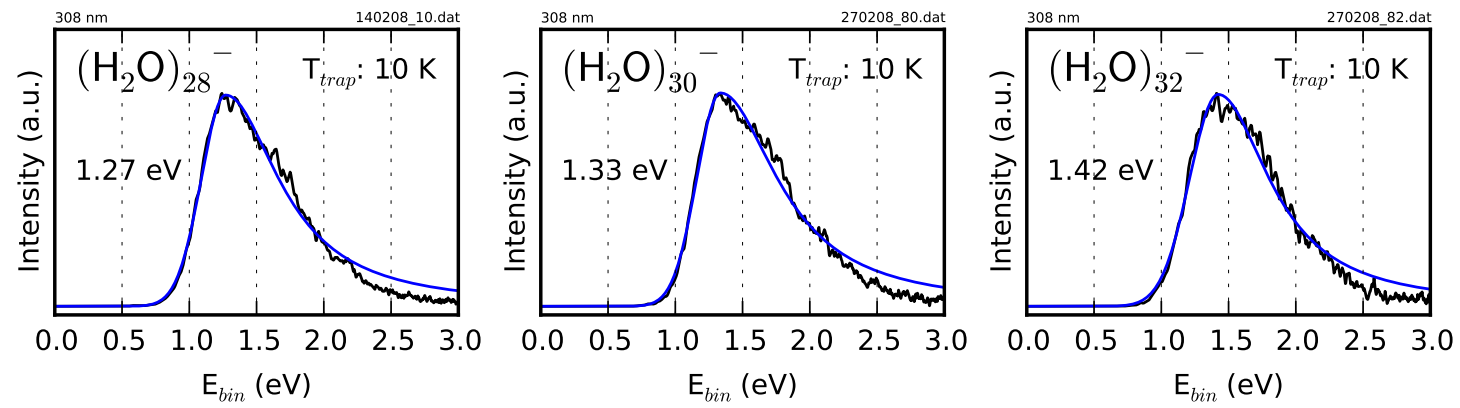

Figure 4.7.: Example photoelectron spectra from the size range $n=28$ to 33 . 


\section{Sizes 34 to 49}

In the size range from 34 to 49 the low binding energy slope also start to deviate from a single GL function. The reason for this is a steeper onset and flattened top region of the peak (fig. 4.8). The maximum moves with growing cluster size to higher binding energies in respect to the peak base. This leads to a more and more triangular peak shape, the larger the clusters become.
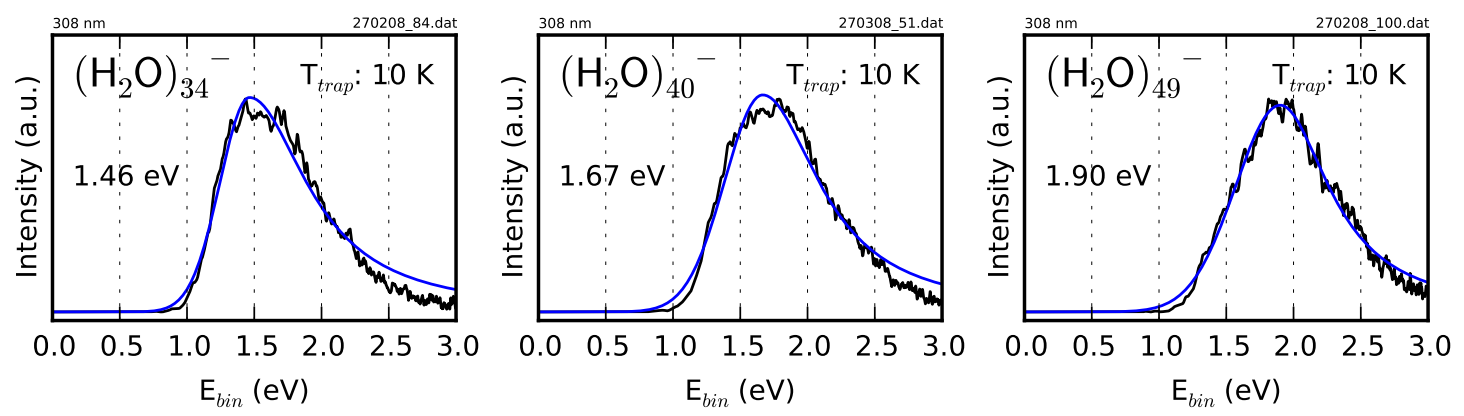

Figure 4.8.: Example photoelectron spectra from the size range $n=34$ to 49 .

Most spectra in this size range show a strong temperature dependency, in particular $\left(\mathrm{H}_{2} \mathrm{O}\right)_{48}{ }^{-}$. The previously described spectral changes with the cluster size, especially the shift of the maximum in respect to the base, can also be found for the spectra of one cluster size at different temperatures. This is demonstrated in fig. 4.9 using the $\left(\mathrm{H}_{2} \mathrm{O}\right)_{48}{ }^{-}$cluster as an example. The considerable shift of the (fitted) peak maximum of about $0.2 \mathrm{eV}$ is, however, partially caused by the bad conformity of the single GL with the experimental data.

The main spectral shifts can be assigned to roughly 3 temperature intervals: $10 \mathrm{~K}$ to $80 \mathrm{~K}, 80 \mathrm{~K}$ to $140 \mathrm{~K}$, and $140 \mathrm{~K}$ to $300 \mathrm{~K}$. A more detailed analysis will follow later in sec. 4.2.6.
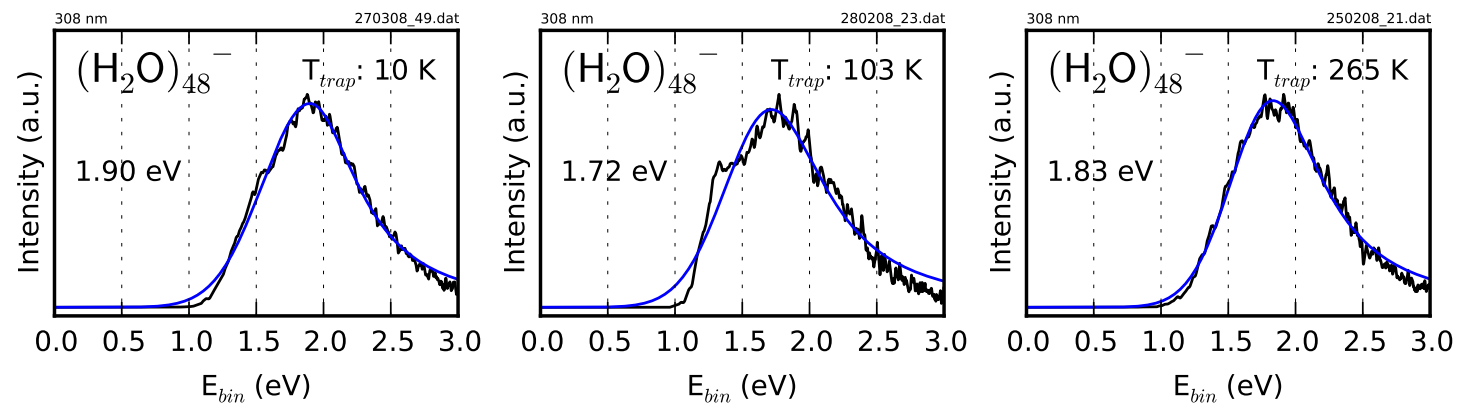

Figure 4.9.: Photoelectron spectra of $\left(\mathrm{H}_{2} \mathrm{O}\right)_{48}{ }^{-}$measured at different temperatures, demonstrating the impact of the cluster temperature on the line shape. 


\section{Sizes 50 to 53}

The spectra of the sizes 50 and 51 show for low temperatures $(<160 \mathrm{~K})$ again the "classical", asymmetric shape, with a steeper low binding energy slope and a moderately declining high binding energy slope (fig. 4.10). In the high temperature range the peak shape becomes more symmetric, triangular like, because the moment of the Gaussian part $\sigma_{G}$ grows faster with the temperature, than $\sigma_{L}$. The overall agreement with a single GL is, like for the small clusters, in principle quite good. Solely the Lorentzian slope diverges slightly.

The peak form changes considerably for the next two sizes, 52 and 53, which have a distinctive triangular shape. This leads to a particularly bad agreement of the $\left(\mathrm{H}_{2} \mathrm{O}\right)_{52}{ }^{-}$clusters experimental data with a single GL fit. Although the $\left(\mathrm{H}_{2} \mathrm{O}\right)_{53}{ }^{-}$curve fits better due to its less meandering slopes, its $10 \mathrm{~K}$ spectrum shows a tiny shoulder on the onset of low binding energy slope (fig. 4.10, right), which is naturally not mapped by a single GL fit.
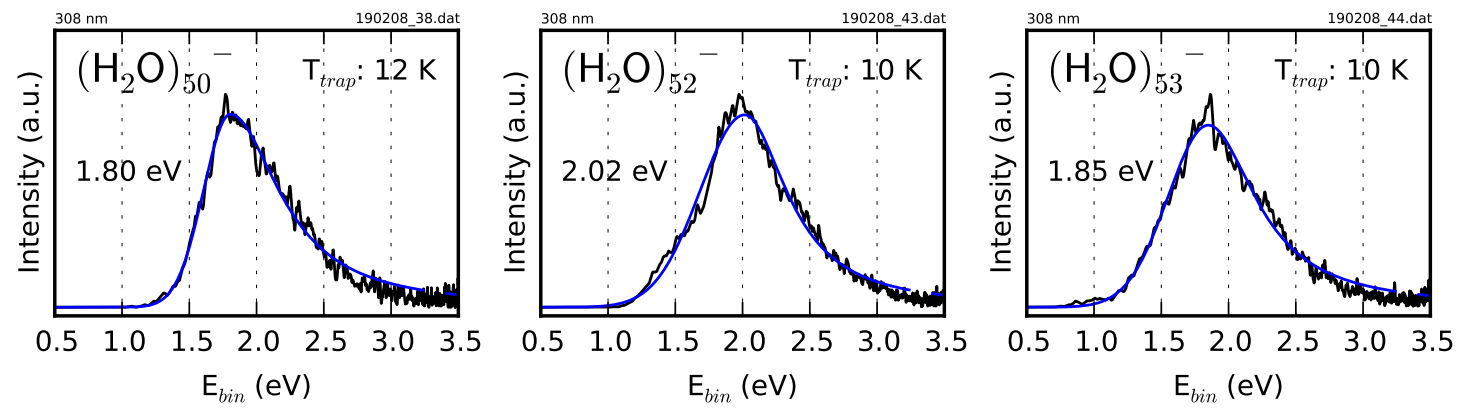

Figure 4.10.: Example photoelectron spectra from the size range $n=50$ to 53 .

\section{Sizes 54 to 66}

A single GL function fits the spectra in the size range from 54 to 66 water molecules generally quite well. All sizes still show, however, a visible deviation at the point, where the high binding energy slope fades away. The deviation of the low binding energy slope is very small and only observable for few sizes (fig. 4.11). The temperature dependency of the spectra turns out to be rather moderate.

The sizes 54 and 55 have still a more asymmetric peak shape. The low binding energy slope deviates at several points from the single GL and the pointy peak maximum is not represented entirely by the fit. The larger sizes, starting at 56, return to a more symmetric peak shape. The spectra of the sizes 58 to 61 and 63 feature an onset of the raising slope, which is too steep to be matched well by a single GL (cf. fig. 4.11, middle and right plot). 

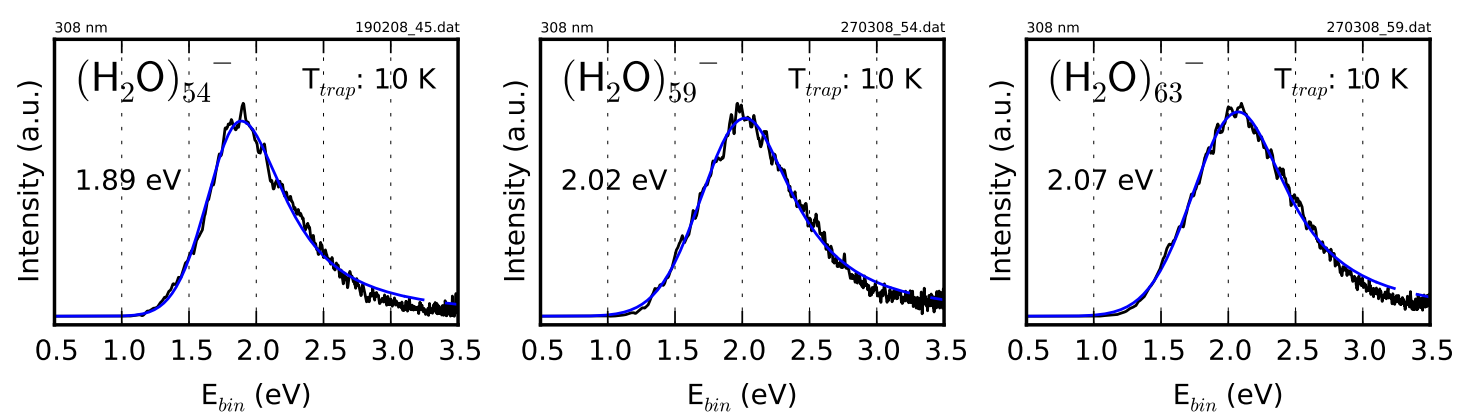

Figure 4.11.: Example photoelectron spectra from the size range $n=54$ to 66 .

\section{Sizes 67 to 1100}

In the size range 67 to 80 , the deviation on the low binding energy slope onset remains barely visible for few size only. It vanishes completely for sizes larger than 80 water molecules. The typical high binding energy slope deviation is also pretty low, but is still distinguishable up to size 115 (fig. 4.12, left plot). All spectra of clusters consisting of more than $115 \mathrm{H}_{2} \mathrm{O}$ molecules are very well represented by a single GL function. For spectra without subtracted background, the Lorentzian tail becomes increasingly superimposed by the detector artifact, the more the cluster grows (fig. 4.12, middle and right plot).
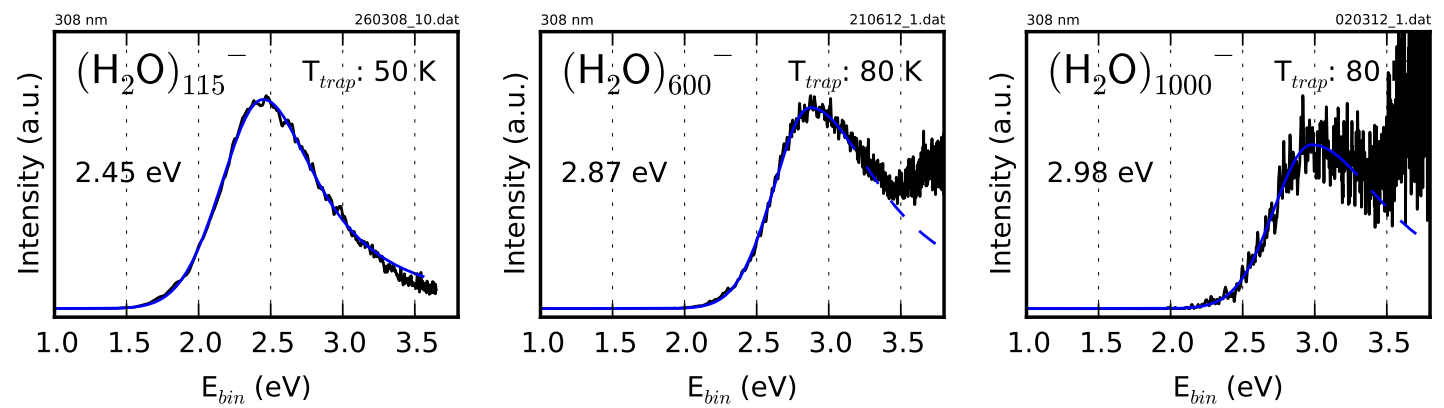

Figure 4.12.: Example photoelectron spectra from the size range $n=67$ to 1100 .

\subsubsection{Results of the Single GL Fit Analysis}

The peak analysis of the previous section has shown that the congruency of the experimental spectra with a single GL fit depends strongly on the cluster size. Basically all sizes $<67$ show considerable deviations. They become much smaller in the size range from about 67 to 115 water molecules and virtually nil for sizes larger than 115.

Another finding is the varying peak shape for different cluster sizes: they shift from a distinct asymmetry to more symmetric, triangular forms or peaks with too 
wide tips or even flat tops. By fitting them with a single GL function, the resulting curves exhibit large shifts of the peak maximum in respect to its base, expressed by changes of the width parameters $\sigma_{G}$ and $\sigma_{L}$. Although for many sizes this is due to their actual peak shape, there are also several sizes, where it is an artificial effect caused by the bad agreement of the fit.

The evolution of the peak maxima and the peak shape parameter is comprehensively depicted by plotting them against the cluster size. The dielectric sphere model (cf. chapter 3) establishes a linear relation between the VDEs and the reciprocal cluster radius (eq. (3.2) ), which suggests plotting them against $n^{-1 / 3} \propto 1 / R$.

The position of the peak maximum is usually considered as the vertical detachment energy (VDE) or binding energy of the excess electron of the water cluster anion. The upper panel of fig. 4.2.3 shows a plot of the VDEs gained from the single GL fits of all recorded spectra. In the size range from 15 to 30 increases the VDE clearly in a nonlinear fashion. The size ranges 30 to 80 and 80 to 1100 display in good approximation a linear growth, but with different gradients. The sizes 20 and 52 show larger shifts in their VDE in comparison to the neighboring sizes. The measured VDEs of a single size do not result in a sharply defined value, but rather form a band with a width of about $100 \mathrm{meV}$ to $250 \mathrm{meV}$.

In the lower panel of fig. 4.2 .3 the peak widths ( fwhm, cf. eq. (4.3)) and width parameters $\sigma_{G}$ and $\sigma_{L}$ are plotted versus the inverse cluster radius. Whereas $\sigma_{L}$ is identical the width of the Lorentzian part, $\sigma_{G}$ is only proportional to the width of the Gaussian part by a factor $\sqrt{2 \ln 2} \approx 1.18$. The evolution of each parameter is, however, better distinguishable in the plot, when using the width parameter instead of the actual partial width.

The fwhm raises quickly from approximately $400 \mathrm{meV}$ to $600 \mathrm{meV}$ in the size range 15 to 21 . It continues to raise more slowly to $800 \mathrm{meV}$ for the sizes 22 to 40 and basically stays at this value up to size $150(850 \mathrm{meV})$. For large clusters with more than 150 water molecules, the value of the peak width fluctuates considerably between $0.8 \mathrm{eV}$ to $1.1 \mathrm{eV}$, the mean values, however, seem to slightly increase from about $850 \mathrm{meV}$ to $950 \mathrm{meV}$. The size 21 and the size range between 48 and 58 exhibit particularly strong variations of the width.

The width parameters $\sigma_{G}$ and $\sigma_{L}$ evolve quite differently with the cluster size. Both gain width at the same rate for small clusters from 15 to 25 . From 25 and onwards $\sigma_{L}$ increases much stronger than $\sigma_{G}$, and reaches a local maximum around 35 . Then it declines slowly until it reaches $\approx 45$. It stays more or less constant up to size 100, where it starts to grow again up to 1100 . In this last size range the values fluctuate considerably. $\sigma_{G}$ reaches a local maximum around size 48 with $\approx 0.36 \mathrm{eV}$. The general trend for larger clusters is a slight decrease until it reaches $\approx 0.27 \mathrm{eV}$ at size 1000 . The sizes 21 and 48 to 58 stick out due their large shifts and broad variation. The divergences of the width found for those sizes in the previous paragraph are therefore mainly caused by the fluctuations of $\sigma_{L}$.

Figure 4.14 shows the VDEs and peak shape parameter for the size range 40 to 

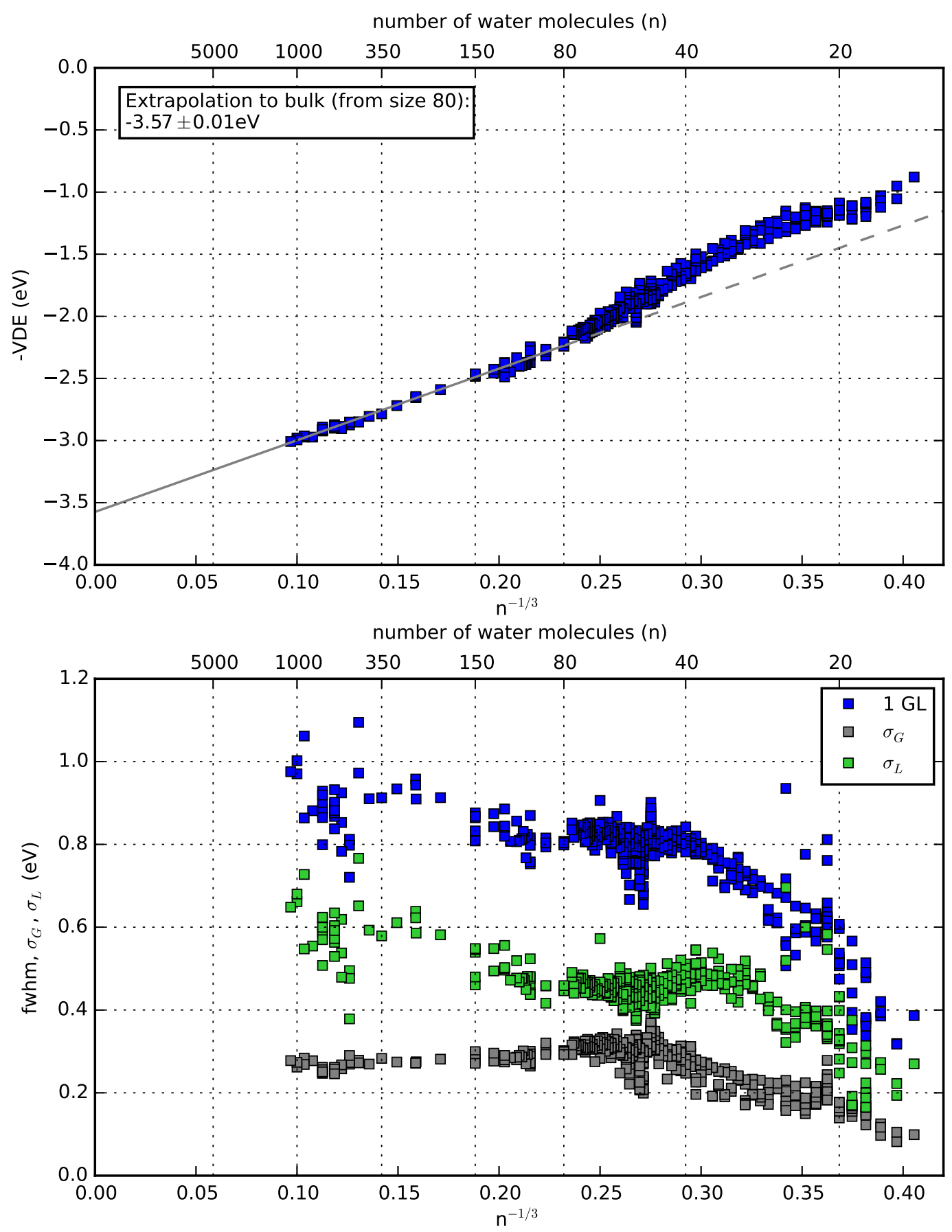

Figure 4.13.: Progression of the VDEs (upper panel) and the width parameters fwhm, $\sigma_{G}$ and $\sigma_{L}$ (lower panel) with the inverse cluster radius $n^{-1 / 3}$. 
60 in greater detail. In the size range from 50 to 60 the VDEs exhibit a strong odd-even staggering, whereas the even sizes show higher binding energies than their neighbors. A similar development from one size to the next can be found for $\sigma_{G}$ and $\sigma_{L}$ in that range. Size 39 shows a small deviation of the VDE as well as of the width parameters. It also exhibits a stronger tendency towards a triangular shape.
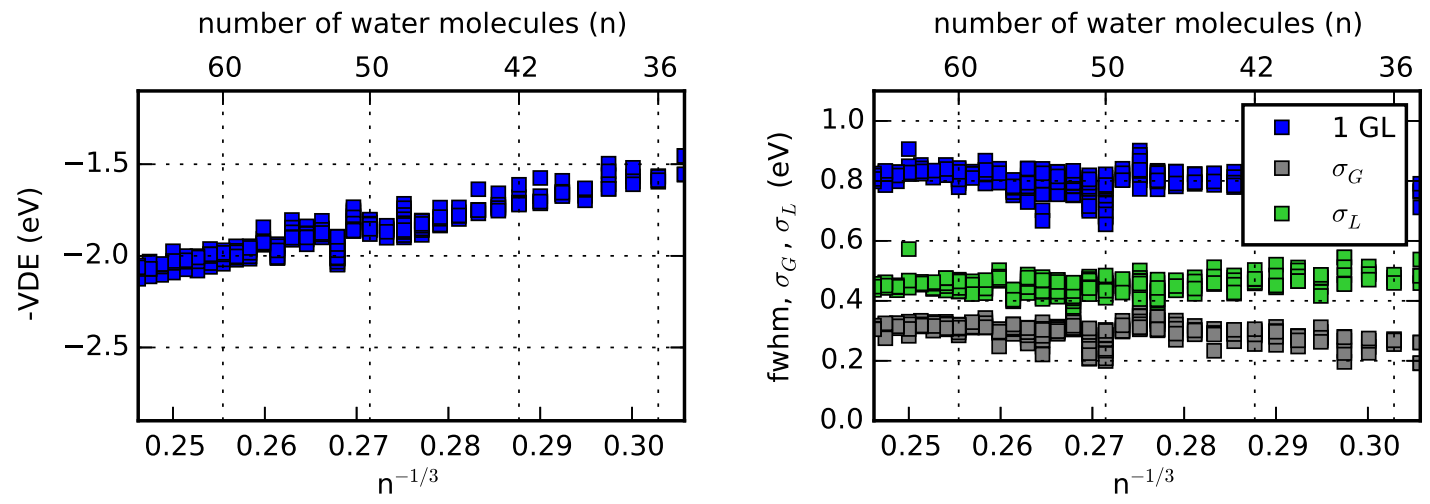

Figure 4.14.: Detailed plot of the size region around 50 to 60, which features strongly fluctuating VDEs (left) and peak shape parameters (right).

\subsubsection{Discussion Single GL Fit Results}

The main result of the single GL fit analysis is that both, a linear increase of the VDEs with cluster size and a constant evolution scheme of the peak shape, start only at larger cluster sizes, approximately around 80. For smaller clusters the peak shape is constantly shifting, which becomes particularly evident between 50 and 60 . In the case of $\left(\mathrm{H}_{2} \mathrm{O}\right)_{21}{ }^{-}$the unusual values are clearly caused by the additional peak in front of the main peak: the fit adapts by widening the Gaussian part, which in turn leads to the greater width of the peak in comparison to its neighbors. The larger $\sigma_{G}$ also causes a small shift of the peak maxima to higher binding energies.

From about 60 up to the largest measured cluster with $1100 \mathrm{H}_{2} \mathrm{O}$ molecules, the $\sigma_{G}$ stays almost constant while $\sigma_{L}$ fluctuates rather strongly. This leads directly to wide variation of the width. The reason for this lies in the uncertainty of the Lorentzian slope, which in turn is caused by the high noise and the detector artifacts in the high binding energy region by which the spectra of this size range are especially effected. Nevertheless show the VDEs themselves little fluctuations. This demonstrates that one clearly defined slope, the Gaussian one, defines the maximum quite well.

As we have seen so far, the shifting of the peak shape for the sizes up to $80 \mathrm{H}_{2} \mathrm{O}$ molecules is not only caused by the variations of the spectra, but is amplified by the unfavorable agreement of the data with a single GL functions. For large clusters (> 
80) the fit is, however, very good. Nevertheless is the peak shape still changing. To elucidate this development, fig. 4.15 combines the locations of the curve maximum (VDE) and the locations where the curve reaches half the maximum height (where the width is determined) in one plot. Each set is extrapolated by a linear fit including data from size 80 and larger. The peak maxima move towards the lower binding energy slope with increasing cluster size, thus increasing the asymmetry of the peak. The $\sigma_{L}$ data should be considered cautious due to the decreasing quality of the high binding energy experimental data. The standard deviation given in fig. 4.15 probably underestimates the error of the extrapolation. On the other hand the width of the Gaussian slope is well defined by the experimental data. Its progression converges slightly at the progression of the VDEs, thus further increasing the asymmetry. This is an unexpected size dependent evolution, since the processes causing a broadening of the peak shape should gain from higher number of base units in the clusters (e.g. more possibilities of structures contributing to an isomer class). This may indicate that the single fit description is an incomplete approximation even for those large sizes, although the peak shape is mapped very closely.

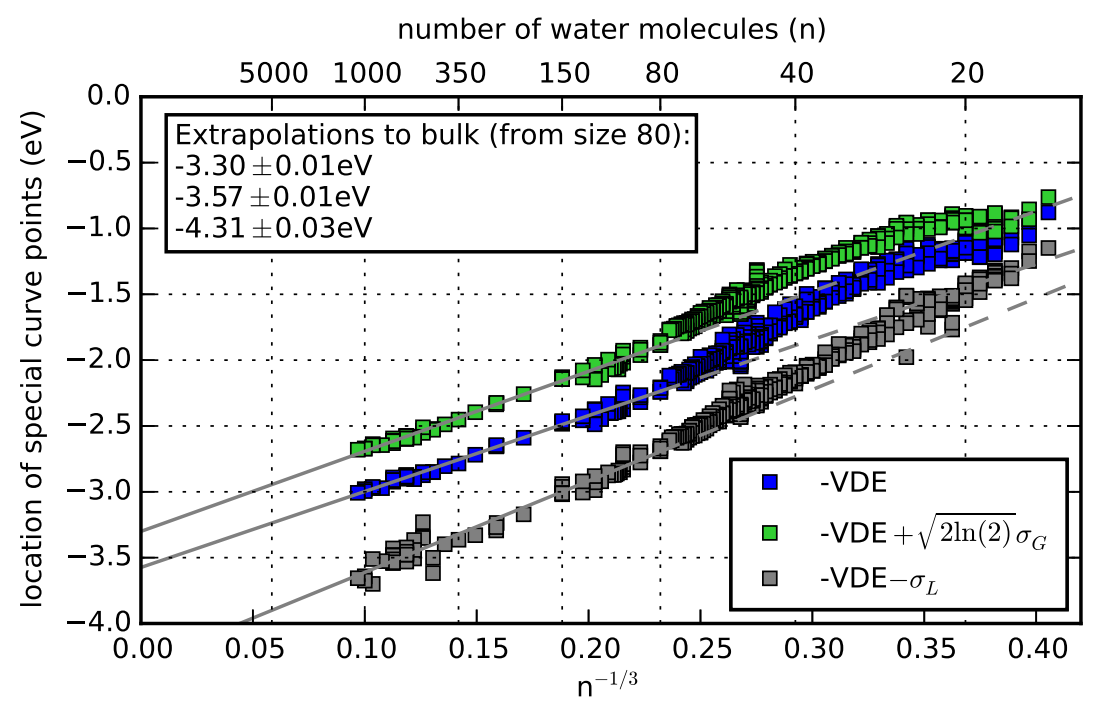

Figure 4.15.: Plot of the VDEs of water clusters including the curve points at half the maximum hight of the GL function.

The single fit approximation turned out to be especially inapt for the size range 30 to 50, which raises the question how to interpret the resulting peak maximum in that range. The position of the maximum lies often between prominent features of the spectrum and is therefore considered best as a weighted average VDE. This leads consequently to a decoupling of the size dependent evolution of the peak base and its maximum, i.e. the onset of the peak follows a slightly different slope, than 
the maximum.

How meaningful are then linear extrapolations to the bulk value under such conditions? Basically two conditions must be fulfilled: the determined peak maximum should represent the maximum of the spectra, i.e. its VDE, and the maxima should exhibit a linear evolution with the cluster size. Considering the current results, this seems to be fulfilled only for sizes larger than $\approx 70$. The gray line in fig. 4.2.3 indicates a linear fit to the VDEs of large cluster sizes starting at 80 . The inflection point at 80 was ascertained by repeatedly fitting the large cluster sizes and including each time one additional smaller size into the fit. For sizes $>80$ the fit parameters barely change, whereas when including sizes $<80$ the fit parameters start to evolve in one single direction resulting in ever steeper gradients and lower axis intersections. The cluster sizes $<80$ feature linear progressions only certain size ranges. The sizes marking these sections are roughly 19, 26, and 80 .

The measured VDEs of most sizes between 15 and 150 cover a band of about $100 \mathrm{meV}$ to $250 \mathrm{meV}$, which expresses the shift of the peak maxima for each size. The reasons why the fits for a single size result in such a broad distribution of binding energies are manifold: i) first of all, the cluster temperature influences the peak shape, ii) in case of a bad approximation of the experimental data by a single GL function, may a small variation of the experimental spectra lead to considerable shifts of the maximum position, iii) even for a good agreement and at a fixed temperature one finds small shifts, which can be either artificial due to the statistical nature of the experimental spectra or are indications that the cluster formation process influences the cluster shape, despite the thermalization process (incomplete annealing).

Since the VDE plots contain data from cluster sizes with many as well as with few temperature dependent measurements, it should be highlighted that the sizes with many (temperature dependent) measurements show generally a broader variation of the binding energy. However, it becomes clear from sizes with many temperature measurements that the width of the binding energy spread is ultimately defined by the temperature dependent evolution of the PES for each particular size. This includes of course also artificial shifts due to badly fitting functions. Since the influence of the temperature has a considerable impact on the fit results, it will be discussed in a separate section (cf. sec. 4.2.6).

\subsubsection{Refinement: Multi GL Fit}

One of the main findings of the peak analysis in the previous section, is the bad approximation of the experimental spectra with a single GL functions for many sizes. There is the trivial case of a distinct second peak $(21,26,53)$. The obvious case, where the peak shape just can not be represented by a single GL (many sizes between 30 to 50 and 52). And less clear cases in varying degrees, where a part of one slope is not quite captured by a single GL. 
As the cases of the sizes exhibiting pre-peaks have demonstrated, is the accuracy of the Gaussian slope high enough to expose even small derivations of the fit function from the experimental data. The Lorentzian slope should be treated more carefully in this respect, especially for the large cluster sizes.

If we keep the assumption that the GL function is principally a good representation of the photoelectron spectra of water clusters, the next step in improving the analytical representation of the experimental data, is to use more than one GL function for the fit. Besides the small, well separated peak in front of the main peak, visible for few sizes, it is a priori not clear how many GL function should be used for a fit of the main peak. Since fitting the spectra with GL functions is an empirical approach in the first place, the "right" number of Gaussian-Lorentzians can only be determined as the (minimum) number of functions to gain a qualitative better agreement of the fit with experimental data. As we will see in the following section, this is harder to implement as it may seem on the first glance.

\section{Two GL Fit Analysis}

Since the number of GL functions is not easily determinable, we can start by fitting the main peak with 2 GL functions. In general this greatly improves the agreement of the fit with the experimental data for all sizes up to approximately 60 water molecules. For clusters between approximately 60 and 350 molecules the improvement is less pronounced and for many sizes there are two possible variants to fit the data. For clusters larger than 350 a fit with 2 functions does not improve the result, but rather leads to (artificial) fluctuations of the peak parameters.

Figure 4.16 shows a selection of spectra from the range between 15 to 59 water molecules. This is the range, which profits most from using 2 GL functions for fitting the main peak. The spectra demonstrate also that the intensity maxima of the two GL functions evolve in that range following a specific growth scheme: Around 20 the low binding energy peak is dominant and the high binding energy peak has only a small contribution to the Lorentzian tail of the envelope. With growing cluster size, the maximum of the high binding energy peak increases until it reaches parity with the first peak around 46. Now the low binding energy peak maximum starts to decrease and the higher binding energy peak becomes the dominant one.

An exception to this growth trend are the sizes 50 and 51. There low binding energy peak is either non existent or has very low intensity. Instead they prefer an additional peak on the higher binding energy side (cf. fig. 4.16, lower row, middle panel). The following sizes continue to follow the general trend again, but the lower binding energy peak of 52,53 , and 56 is particularly well distinguishable.

Starting with some spectra in the size range from 50 to 60 and especially for sizes larger than approximately 60 it is often possible to fit the data in two ways: a dominant peak located near the maximum spectral intensity and a smaller peak either in front of it (fit variant 1) or behind it (fit variant 2). Some spectra, even 

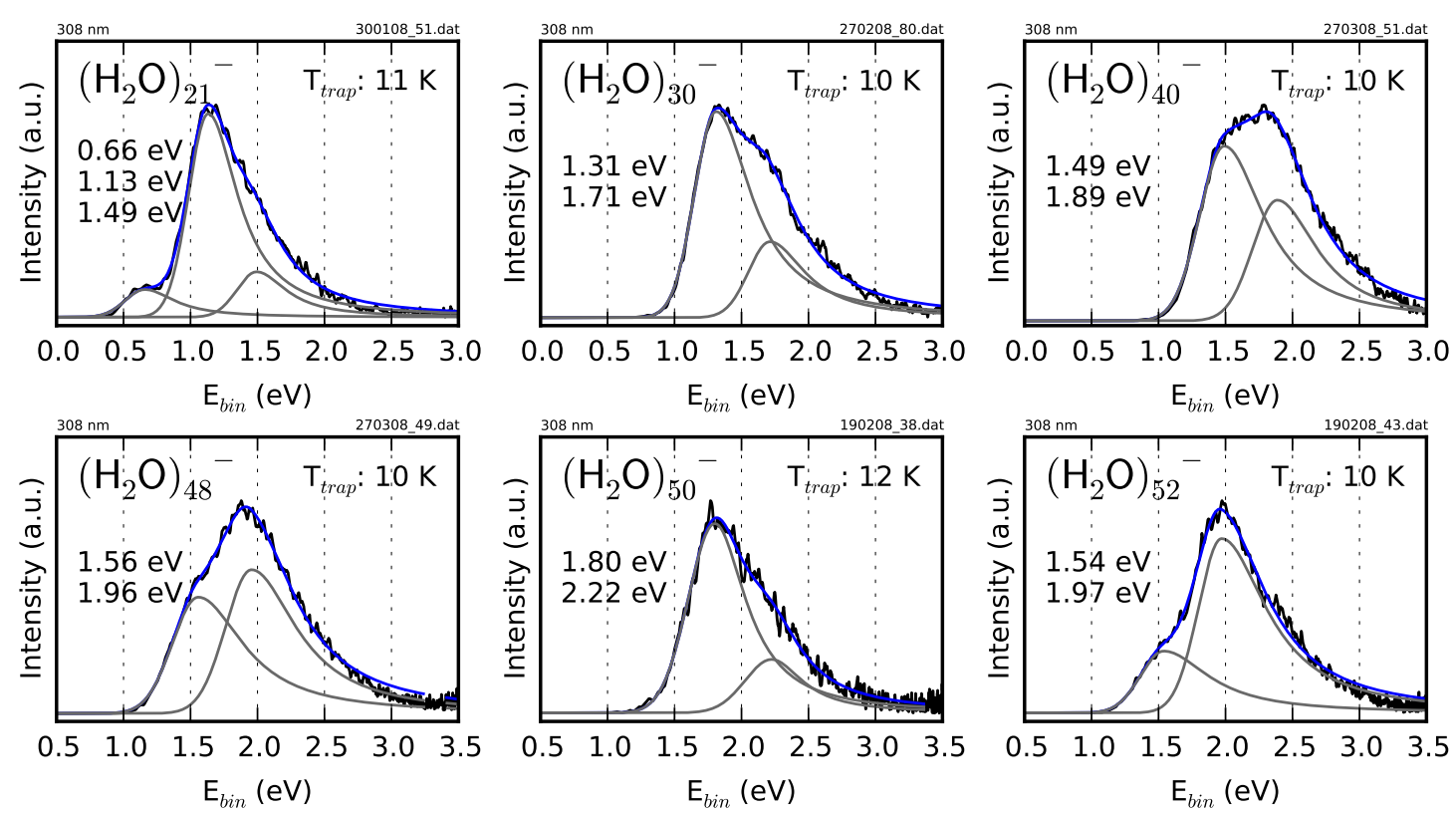

Figure 4.16.: Example photoelectron spectra from the size range 20 to 55, fitted with 2 GL functions. Note the shift of the displayed binding energy range between the upper and the lower row.

for a single cluster size, have a clear preference for one of the fit variants, which makes it nearly impossible to fit the other variant. In most cases the preference can change with the cluster temperature.

Fig. 4.17 demonstrates the case, where both fit variants are basically equally selectable by means of a spectrum of $\left(\mathrm{H}_{2} \mathrm{O}\right)_{60}{ }^{-}$. The right hand plot (fit variant 2) yields qualitatively a better fit, since the Lorentzian slope is better approximated. The left hand version (fit variant 1) shows a marginal better approximation of the Gaussian slope. Looking at the fit parameters both variants result in different values. The location of the peak maximum of the main peak differs by $0.06 \mathrm{eV}$. The width parameters of the left variant are $\sigma_{G}=0.21 \mathrm{eV}$ and $\sigma_{L}=0.44 \mathrm{eV}$, yielding a width of fwhm $=0.68 \mathrm{eV}$. Whereas the right variant results in $\sigma_{G}=0.31 \mathrm{eV}$, $\sigma_{L}=0.28 \mathrm{eV}$, and fwhm $=0.65 \mathrm{eV}$. This means that fit variant 2 results in GL functions with a "reversed" asymmetry, where the width of the Gaussian part is larger than the width of the Lorentzian.

In general, both variants result in slightly different peak maximum locations. The effect on the shape parameters, however, is not always as strong as in the discussed example.

For larger clusters, starting at 100 to 150 water molecules, it becomes increasingly difficult to fit variant 1 , whereas variant 2 works slightly better. Water clusters containing more than 350 molecules can not be fitted meaningfully by either variants, 

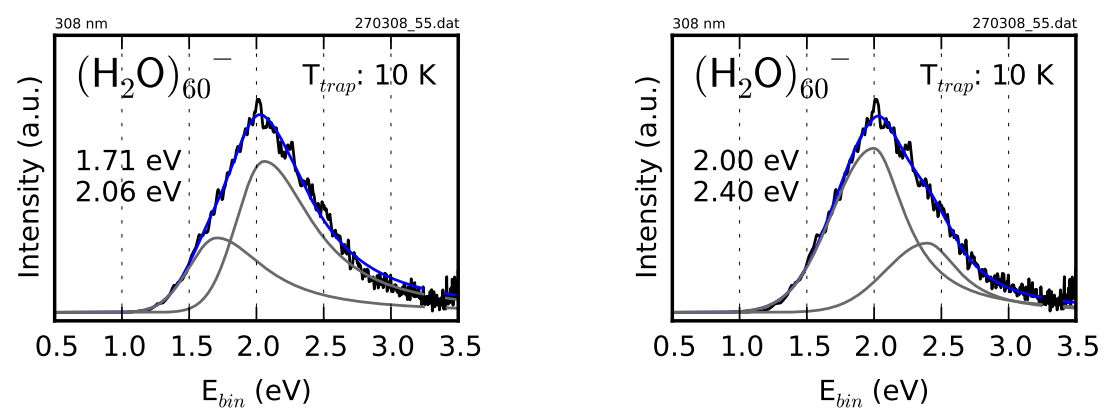

Figure 4.17.: The two possible variants to fit the photoelectron spectra of a medium sized water cluster with 2 GL functions, demonstrated for $\left(\mathrm{H}_{2} \mathrm{O}\right)_{60}{ }^{-}$. Left: fit variant 1, right: fit variant 2.

because this leads to very strong fluctuations of the resulting VDEs.

The upper panel of fig. 4.18 shows the evolution of the VDEs with the cluster size for fit variant 1 . Up to $\approx 47$ the binding energies show two clearly separated bands. The few spectra with unusual large widths or $\sigma_{L}$ respectively, are measurements taken at $2.1 \mathrm{eV}$ photon energy $(590 \mathrm{~nm})$, which means that a part of the Lorentzian slope is missing. The size range from 48 to 80 has 3 bands, of which the middle one is rather broad and splits into 2 sub-bands. The binding energies of the sizes 48 to 60 exhibit particular strong fluctuations. The sizes $>80$ show again only 2 bands. The lower binding energy peak deviates increasingly from a general trend.

In the lower panel of fig. 4.18 is the corresponding evolution of the width parameters plotted. $\sigma_{G}$ shows a steeper increase up to 20 . Then it stays almost constant until it reaches 48 . In the size range between 48 and 80 it shows the largest fluctuations and a moderate increase of about $0.05 \mathrm{eV}$. For sizes larger than 80 , it stays almost constant again. The overall increase from 20 to 1100 amounts to $\approx 0.1 \mathrm{eV}$. $\sigma_{L}$ increases with a much higher rate, with an overall increase in the same size range of $\approx 0.37 \mathrm{eV}$. The slope is approximately linear from 26 to 275 and flattens for sizes $>275$. It shows also huge fluctuations between 48 and 80, and for particularly small $(<22)$ and large $(>350)$ clusters. Since $\sigma_{L}$ is the main contributor to the width, the width increases accordingly: roughly linear at the beginning and then flattens out from 275 onwards.

Figure 4.19 displays the same data but for fit variant 2. The results of the variants start to diverge from each other around size 47. Mayor differences are visible for sizes > 80: $\sigma_{G}$ makes a distinct step at size 80 and increases abruptly. After that it shows a to constant, slightly decreasing progression for all following sizes. $\sigma_{L}$ assumes also an approximately constant value between 80 and 275, although the fluctuations are quite high. It then makes a steep increase of about $0.2 \mathrm{eV}$ from 300 to 1100. The width evolves accordingly and remains narrower in the region between 80 and 350. The differences of the peak parameters resulting from the two 

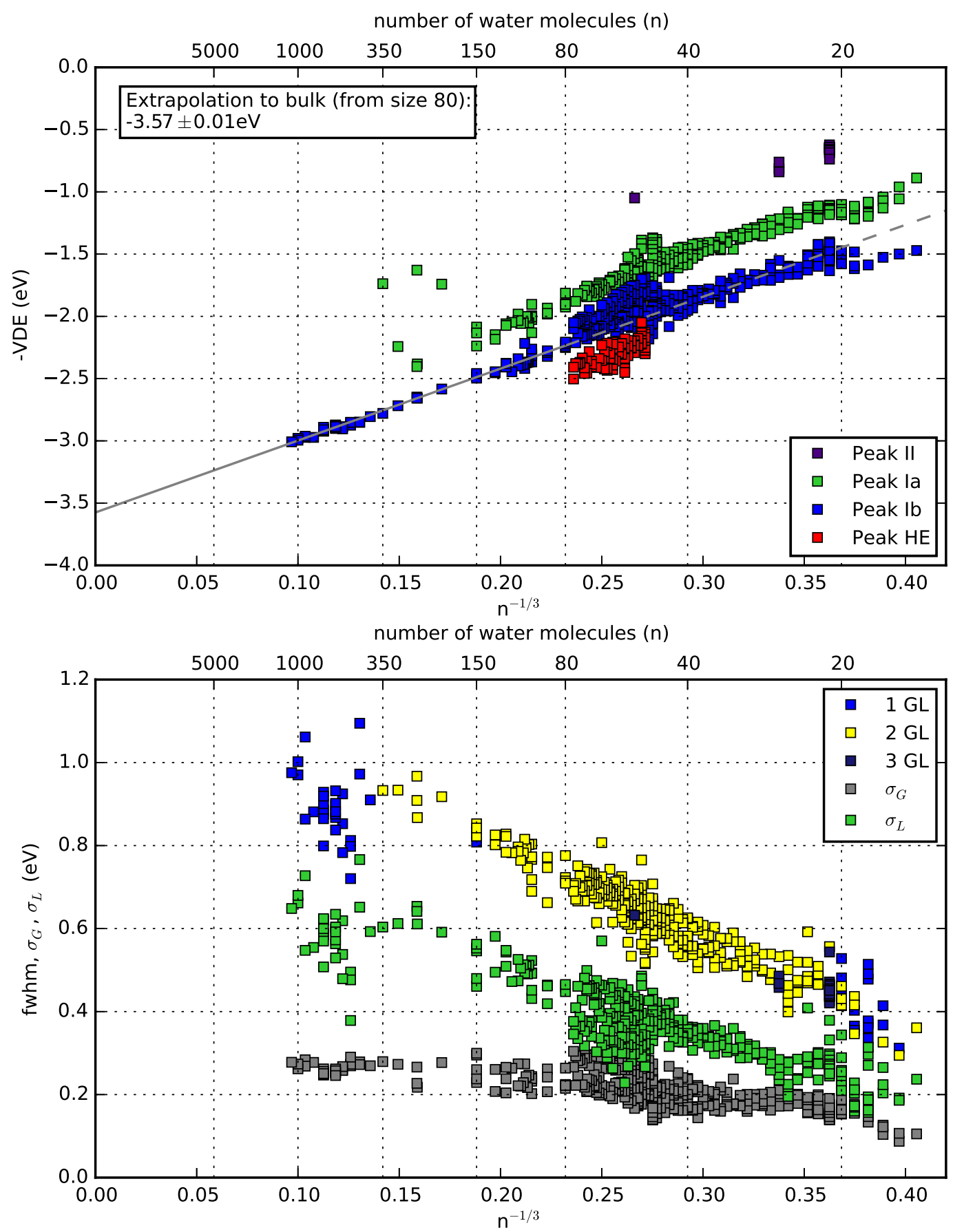

Figure 4.18.: VDEs (upper panel) and width parameters (lower panel) versus the inverse cluster radius $\left(n^{-1 / 3}\right)$ from the 2 GL fit variant 1 . 

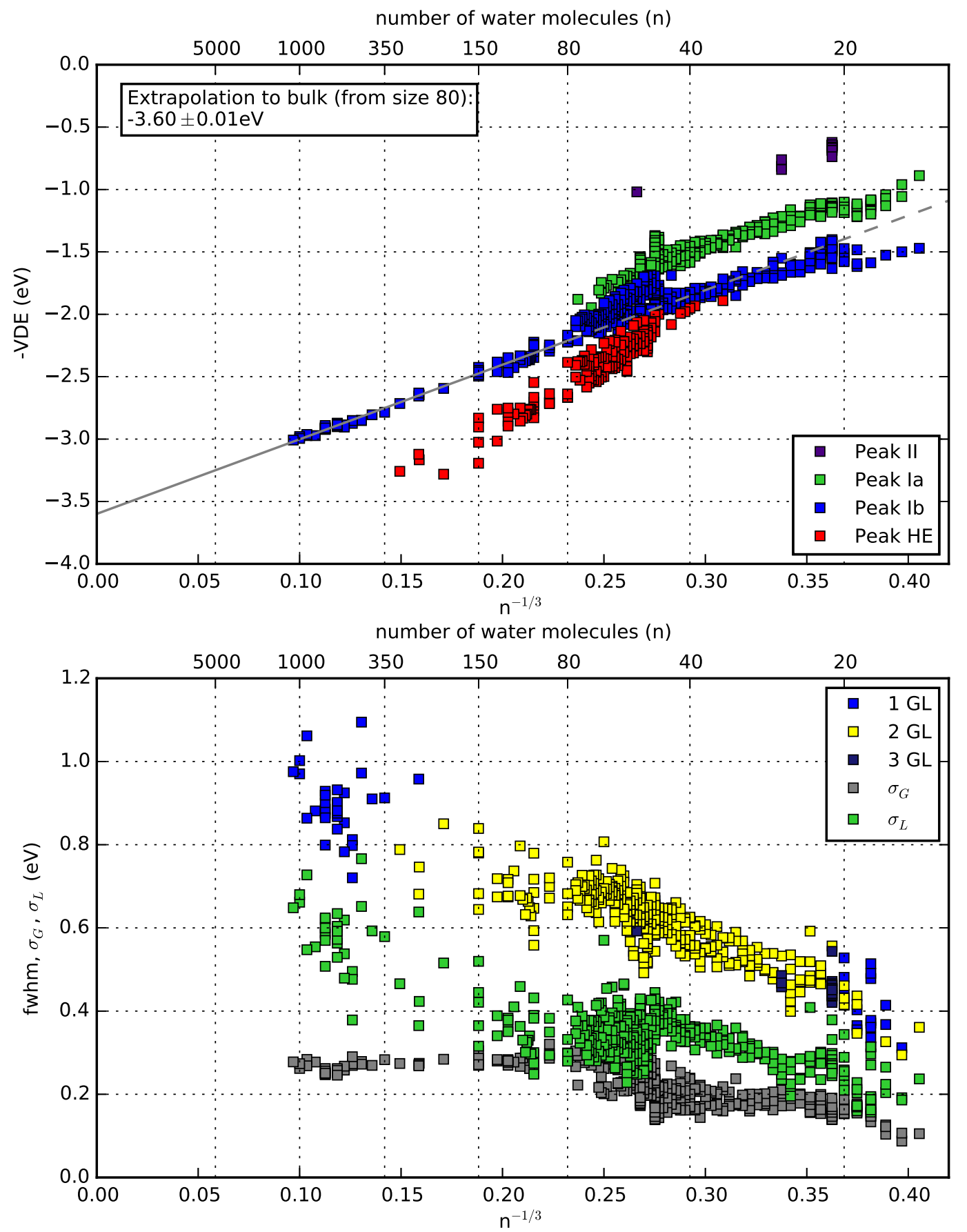

Figure 4.19.: VDEs (upper panel) and width parameters (lower panel) versus the inverse cluster radius $\left(n^{-1 / 3}\right)$ from the 2 GL fit variant 2 . 
fit variants become clearer, when considering only measurements of clusters with low temperatures $(<90 \mathrm{~K})$, as shown in fig. 4.20 .
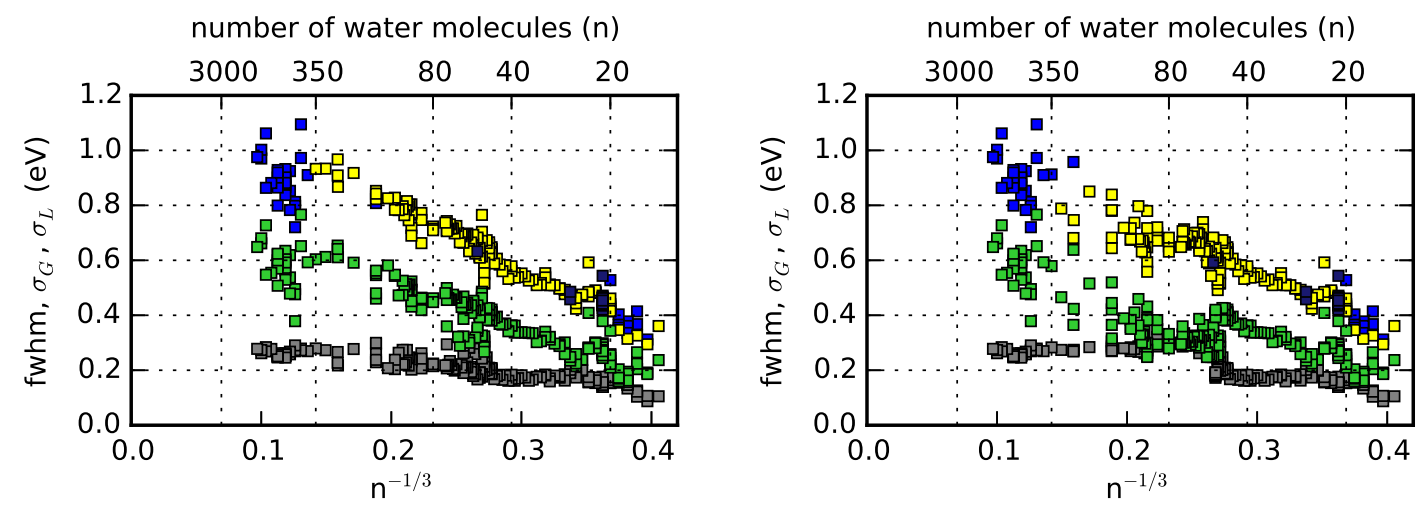

Figure 4.20.: Comparison of the development of the width parameters for low temperature $(<90 \mathrm{~K})$ measurements only. Left: fit variant 1, right. fit variant 2 .

\section{Discussion of the 2 GL Fit Analysis}

Fitting the main spectral peak with 2 GL functions greatly enhances the agreement of the fit with the experimental data, especially for the small and medium sized clusters up to approximately 60. Most of the deviations of the main spectral peak from a single GL function can therefore be attributed to the existence of two peaks. That means that each spectrum is the sum of both peak contributions, which vary in intensity depending on the cluster size and, to a certain degree, on the temperature.

There are still some sizes, which show small deviations: In the size range 30 to 49 is the Lorentzian tail not reproduced completely, the spectra slope down more quickly, than the fit. Between 50 and 80 it depends on which fit variant is used. Thus variant 1 results in a deviation of the high energy tail and variant 2 causes a small disagreement on the low binding energy onset of the peak. The sizes 52 and 53 show stronger deviations even on the mid slopes.

The size range between 50 and 60 still exhibits high fluctuations of the VDEs, even when fitted with two GL function. That range was found to be irregular also in the single fit analysis. However, at that time an artificial cause could not be excluded due to the bad agreement of the fit with the experimental data. When fitted with two GL functions, both peaks shift more or less simultaneously. Since the agreement of the fit is good, this is a strong hint that the shifts are intrinsically to the experimental spectra and not merely changes in the intensity of the two peaks.

The same applies to the temperature dependency of the VDE: the width of the VDE bands remains the same or even increases for the 2 GL fit analysis. This 
suggests that the cluster temperature actually influences the VDEs and that the shifting VDEs are not simply caused by varying peak intensities.

Besides the temperature and the intrinsic VDE shifts of the sizes 50 to 60, contributes the ambiguity of the 2 GL fit also to the particularly broad VDE bands in the size range from 50 to 80 . Especially the sizes which flip between the two fit variants cause an artificial broadening of the bands.

The existence of two fit possibilities in the range 80 to 350 leads to ambiguous results of the determined VDEs. The small shift in the VDEs for both fit variants influences the value of the linear extrapolation more than the standard deviation of the fits: 3.57(1) eV for variant 1 and 3.60(1) eV for variant 2 (cf. fig. 4.18, 4.19).

Both fit variants also result in different peak shapes, as can be seen by comparing the width parameters. In the case of variant $1 \sigma_{L}$ levels out at approximately 300 , in contrast to the continuous rise for smaller sizes. Variant 2 shows a distinct step for $\sigma_{G}$ between 50 and 60. $\sigma_{L}$ shows a dip between 50 and 80 and then steep rise starting at 300 . The discontinuities becomes particularly visible for low temperatures (fig. 4.20).

It remains the question, how to interpret two GL peaks, which form the main spectral peak? Since the peak marked with "Ia" in the VDE plots (fig. 4.18, 4.19) is the dominant peak between 15 and the transition region around 46 and peak "Ib" is the main contributor to spectra with more than 49 water molecules, one can assume that they represent two different isomers. However, for small cluster sizes $<30$ exist an alternative interpretation. Coe et al. assorted the high binding energy peak of these sizes to vibrational excitations[17]. This interpretation was based on a comparison of the energy differences between the two peaks for normal water $\Delta E_{\mathrm{H}_{2} \mathrm{O}}$ and for heavy water cluster anions $\Delta E_{\mathrm{D}_{2} \mathrm{O}}$ (cf. sec. 5.2.2 in the chapter about the deuterated water cluster anions). Fig. 4.21 plots the mean value of the ratio of the energy offsets $\Delta E_{\mathrm{H}_{2} \mathrm{O}} / \Delta E_{\mathrm{D}_{2} \mathrm{O}}$ extracted from our data versus the cluster size. The error bars indicate the standard deviation of the averaging. Between 20 and 27 the ratio amounts to 1.4, which is equivalent to a shift by a factor of $\sqrt{2}$ upon deuteration. The sizes 28 to 32 form a transition zone, where the ratio assumes values between 1 and 1.4. For sizes larger 32 the ratio stays near 1. This suggests that the second peak is of vibrational nature for sizes smaller than 30 and represents an isomer for larger clusters. This coincides with the size range where the intensity of peak Ib starts to increase independently of peak Ia. Why the VDEs for the vibrational peak and the isomer peak Ib respectively have the same value and follow seemingly the same size dependency, remains a curiosity. A possible explanation could be that the vibrational peak and isomer peak Ib actually posses different VDEs, but can not be resolved as individual peaks. By fitting them with a single GL function, the resulting VDE would then represent a weighted average of both contributions. That way, the VDEs would shift continuously from the presumably dominant vibrational peak of small sizes to the newly appearing isomer Ib peak for sizes $>30$. 


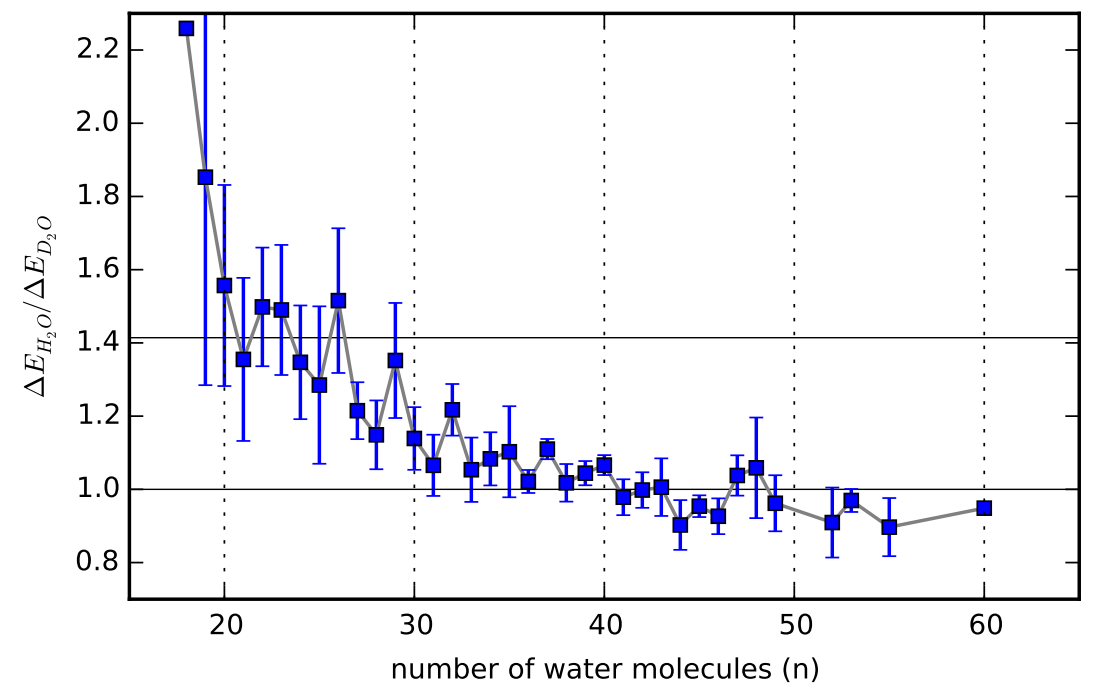

Figure 4.21.: Size dependent development of the ratios of the energy differences between peak Ia and Ib found for $\left(\mathrm{H}_{2} \mathrm{O}\right)_{\mathrm{n}}{ }^{-}$and $\left(\mathrm{D}_{2} \mathrm{O}\right)_{\mathrm{n}}{ }^{-}$.

Although we have reached by now a much better representation of the experimental data by using 2 GL functions, there remain still some discrepancies. The deviations of the 2 GL fit of the spectra of 52 and 53 suggest that the main peak should be rather fitted with $3 \mathrm{GL}$ functions. Using $3 \mathrm{GL}$ functions could also lift the ambiguity, which arises from the try to fit 2 GL functions to triangular-like peak shape in the size range from 50 to 80 . It might also help to clear up the high VDE fluctuations of the sizes 50 to 60 and the discontinuities found for $\sigma_{G}$ and $\sigma_{L}$.

\section{GL Fit Analysis}

Although the spectral line shapes of some sizes exhibit features, which suggests that they are better fitted by 3 GL functions, most other sizes do not show such features. In particular the small sizes from 15 to about 30 are already adequately represented by 2 GL fits. Likewise the very large clusters, starting at approximately 120, are sufficiently mapped by a single GL fit. The intermediate size range contains a core region from about 45 to 75 , which, judging from the comparison of the spectra with a 2 GL fit, would qualitatively profit from a 3 GL fit. To demonstrate the improvement of the fit, three example spectra from that size region are shown in fig. 4.22, This leaves two transitional size ranges, where it is not clear, with how many GL functions they should be fitted.

Since the GL fit is an empirical approach in the first place, we can try different combinations and investigate, how they effect the evolution of the VDEs and the peak shape parameters.

One possible approach could be to increase the number of GL functions, whenever 

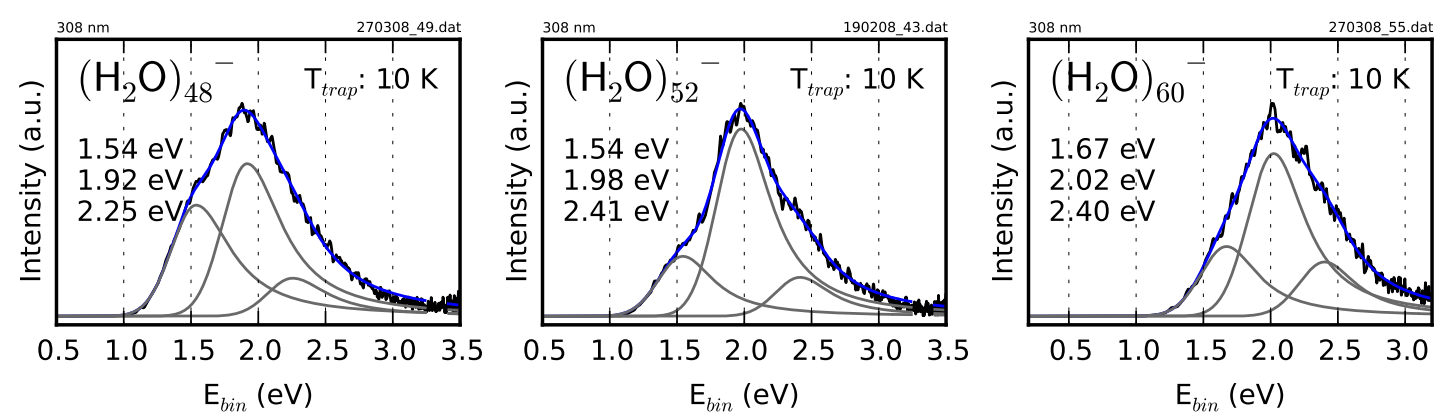

Figure 4.22.: Example photoelectron spectra from the size range 45 to 75 , fitted with 3 GL functions

a qualitative improvement of the fit can be reached with more GL functions, while simultaneously keeping the number of fit functions to a minimum (approach 1). Following this approach, the sizes 15 to 29 are therefore fitted with 2 GL functions, with the exceptions 21 and 26, which are fitted with 3. Sizes 30 to 72 are fitted using 3 GL functions, except 50 and 51, which show already a good agreement with 2 GL functions, and 53, which is fitted by 4 functions, due to its small pre-peak. The sizes 73 to 300 are again fitted by 2 GL functions and all larger clusters with a single function. The results are shown in fig. 4.23 .

The vertical detachment energies form three (occasionally four) bands, which are for most sizes well separated. The development of the dominant peak remains the same as for the 2 GL analysis: Peak Ia is the main peak for small sizes up to 35. Peak Ib continuously grows in height starting around 30, until it has the same intensity as peak Ia around 46. From 50 onwards Ib stays the dominant peak. The size range from 50 to 60 shows still large shifts in the VDEs, the peaks II, Ia, and Ib are, however, more separated, as for the case of the 2 GL fit. The VDEs of peak Ia follow roughly linear trends with increasing cluster size with three different gradients, one for the very small sizes $<20$, one for the sizes 20 to 50 , and another one for 50 to 80 . The same applies to the evolution of peak Ib, only that it shows a fourth trend for sizes larger than 80 .

The width parameters (fig. 4.23, lower panel) show several discontinuities, which are all related to a switch in the number of GL functions used for the fit. Most notably is the reduced peak width, when using 3 GL functions. The discontinuity in $\sigma_{L}$ around size 30 shows that the width changes mainly due to a narrowing of the Lorentzian side of the peak, caused by the additional high VDE peak. The sizes 50 and 51 show a larger width due to being fitted with only 2 GL functions. The increase in width is mainly a result of an increase of $\sigma_{L}$, while $\sigma_{G}$ stays at $0.2 \mathrm{eV}$ in good agreement with the $3 \mathrm{GL}$ results of the neighboring sizes. This supports the decision to use 2 GL functions for the fit, although the cause for the increase in width remains to be determined. At 72 there is a large increase of the Gaussian 

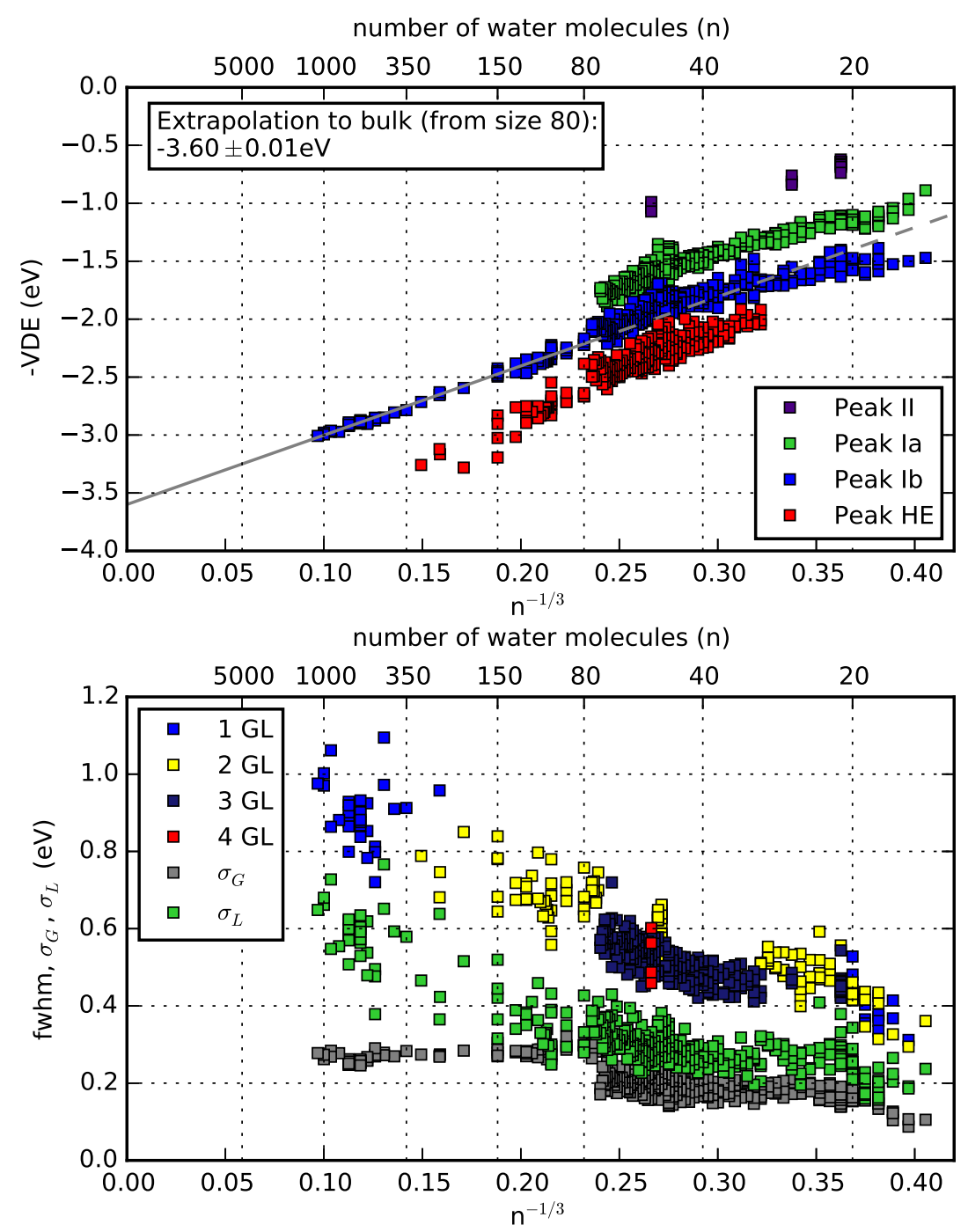

Figure 4.23.: Size dependent evolution of the VDEs (upper plot) and width parameters (lower plot) resulting from the multi GL analysis following approach 1.

width parameter $\sigma_{G}$, which is the main contribution for the sudden increase in the overall width. This changes considerably the peak shape, but seems to have only very little impact on the position of the (dominant) peak maximum. At least no discontinuity is visible in the VDE plot.

The discussed approach is based on indicators found in the experimental spectra when to use 2 or 3 GL functions. These indicators are arguably vague outside the size range 50 to 60 and often rest on the assumption that the high binding energy tail can be mapped accurately with GL functions, which might not be true necessarily. Moreover this method introduces more or less arbitrary switching points, visible as 

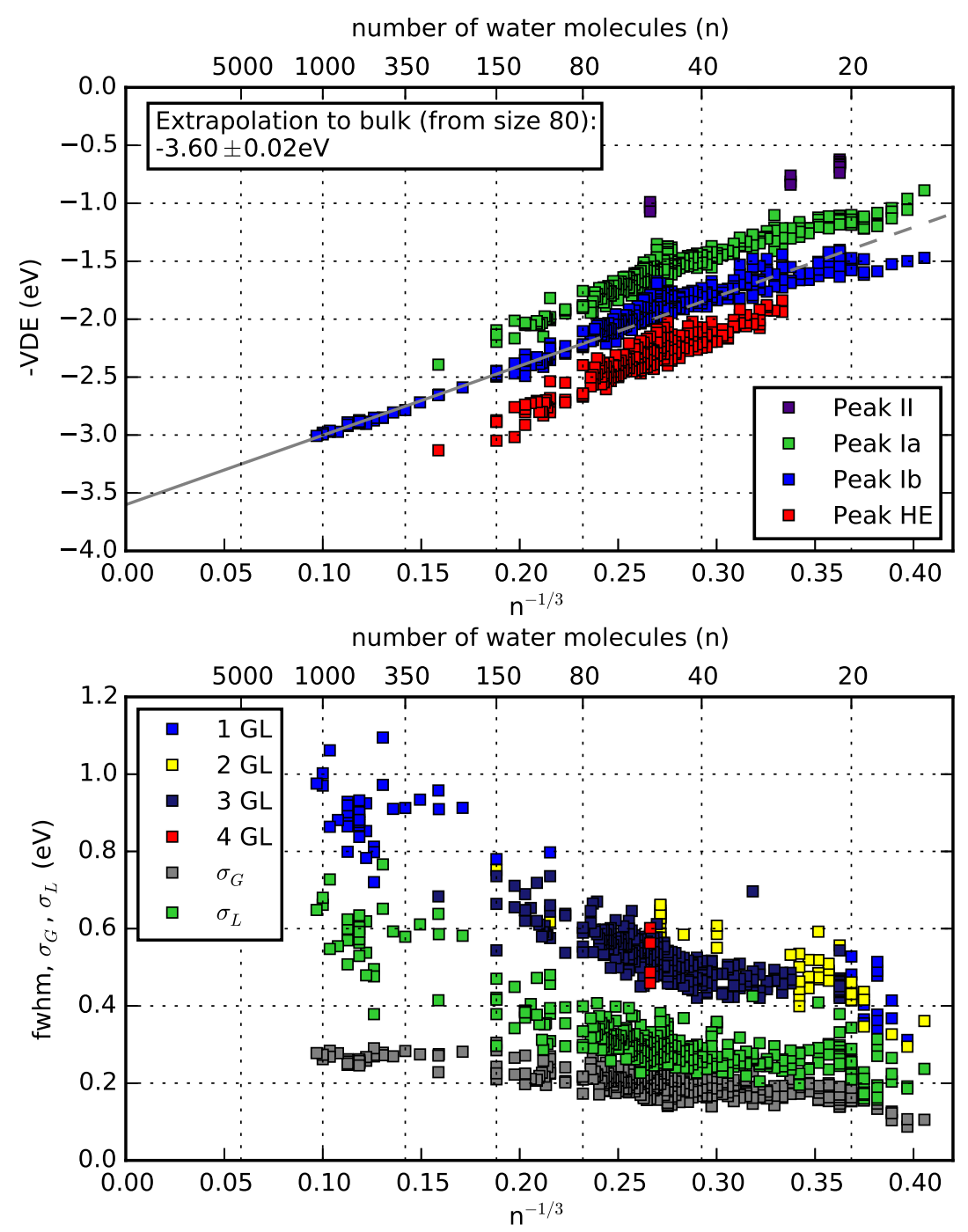

Figure 4.24.: VDEs (upper panel) and width parameters (lower panel) resulting from the multi GL analysis, when an extended size range is fitted with 3 GL functions (approach 2).

discontinuities in the line shape parameter plot. That plot even suggests that the size range to be fitted by 3 GL functions must be extended. To investigate this lead, we have fitted the main peak of all sizes between 27 and 150 with 3 GL functions (approach 2). Smaller and larger size are not fitted easily with 3 GL functions. The results are plotted in fig. 4.24 .

Apart from new VDEs appearing for the additional peaks of sizes formerly fitted with 2 GL functions, primarily the VDEs of peak Ib in the size range 27 to 34 show changes: They show now stronger fluctuations and a small shift to lower binding energies. The peak Ia VDEs remain the same. For larger cluster sizes from 80 to 150 
the impact on the VDEs is virtually nil. It should be noted that the extrapolation to the bulk also yields the same value.

The impact of the extended $3 \mathrm{GL}$ fit on the line shape parameters (fig. 4.24, lower panel) is much clearer. The discontinuities on the switching points are still recognizable although not so drastic. Especially $\sigma_{G}$ shows a smoother evolution. As a result of the extension of the $3 \mathrm{GL}$ fit towards smaller sizes, the full width stays almost constant between 20 and 42 . There is peak around 23 which is clearly related to the 2 GL fit of that size range. The same peak can be found for $\sigma_{L}$. Since for small cluster sizes the third peak appears as a high binding energy peak (peak HE), the changes of the full width are primarily due to changes of $\sigma_{L}$. By contrast, appears for the larger cluster sizes the additional peak on the low binding energy side of the main peak, so that in this range mainly $\sigma_{G}$ changes.

\section{Discussion of the $3 \mathrm{GL}$ Analysis}

Fitting the main peak with 3 GL functions proved to have its benefits, but also demonstrated that it fails to explain the experimental spectra consistently. So do the spectra of some size between 52 and 65 show features which can only be mapped by more than two GL functions. The ambiguity of the 2 GL fit for sizes from 50 to 80 is lifted and the VDEs are better separated, at least for sizes between approximately 37 and 200. On the other hand the development of the peak parameters shows discontinuities whenever a switch of the number of GL functions occurs. Extending the size range fitted with 3 GL functions, only leads to greater uncertainties in the determined VDEs.

In lack of a better solution, we suggest a compromise by using 2 GL functions for the main peak up to size 36, then 3 GL functions up to 200 and 1 GL function for the very large sizes (fig. 4.25). This should provide on the one hand for well separated VDEs and a certain continuity of the peak parameter and on the other hand for a sufficient approximation of the experimental spectral line shape.

Independently of the details of the $3 \mathrm{GL}$ approaches, shows the size dependent development of the VDEs again only a piecewise linear progression, especially for the sizes $<80$. Most notably, the value of the onset of the section containing the largest clusters remains the same, namely about 80 . This means that the shift to a fixed structural growth scheme at this size is fairly decoupled from the peak analysis methods used so far. Consequently we consider it to be inherent to the structural development of the clusters and not an artifact of the analysis.

The same conclusions apply to the VDE shifts in the 50 to 60 size range. The decrease in amplitude when going from 1 GL to 3 GL shows, however, a partial influence by the analysis method.

The assignment of peak $\mathrm{Ia} / \mathrm{Ib}$ to different isomers Ia and Ib remains essentially the same as for the 2 GL analysis. As the comparison with deuterated water clusters will show (cf. sec. 5.2.2), occurs the transition of peak Ib from a vibrational peak 

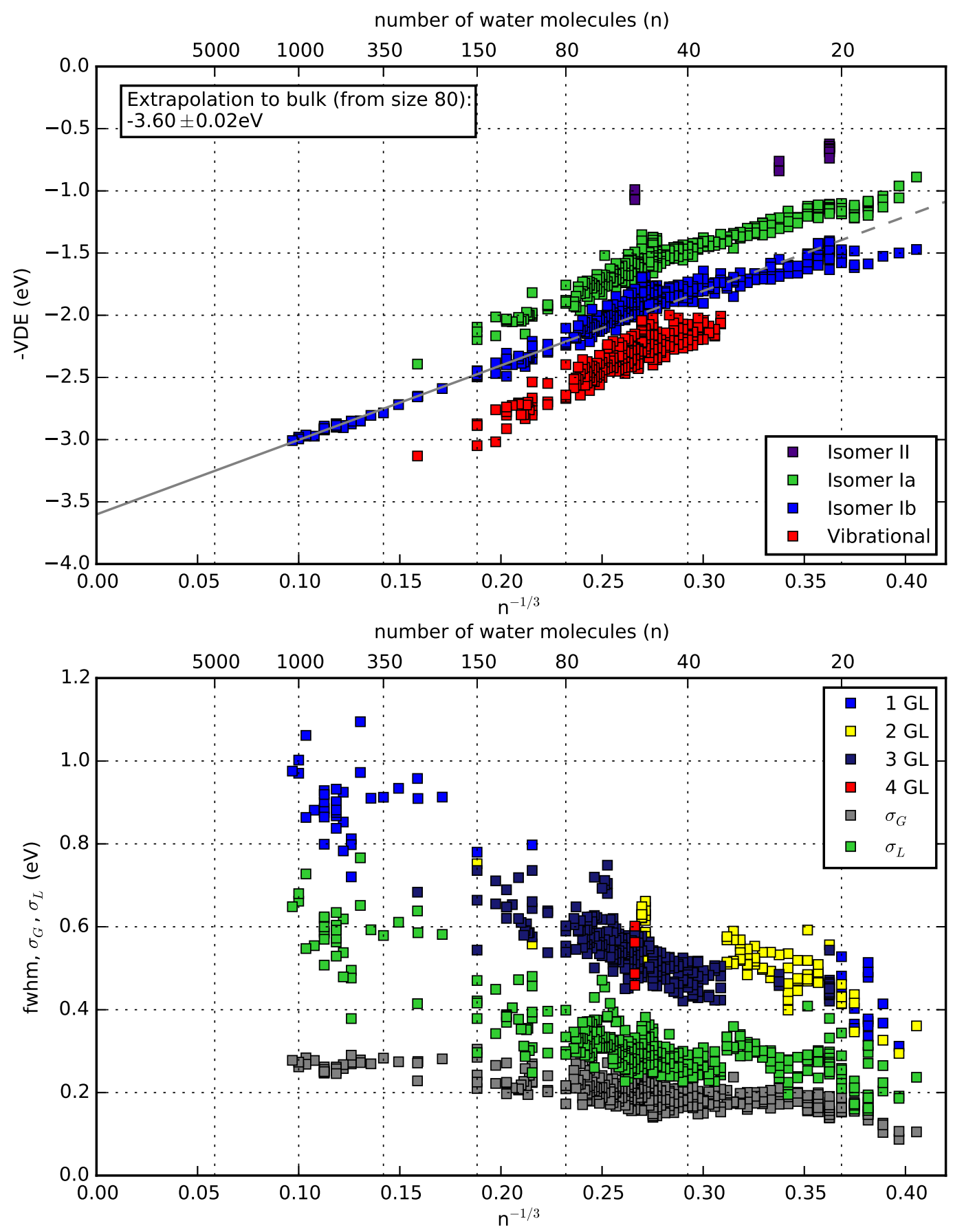

Figure 4.25.: Development of the VDEs (upper panel) and peak width parameters (lower panel) according to the multi GL analysis when using the "best compromise" number of GL functions. 
to isomer Ib at slightly larger cluster sizes. Analogously is peak $\mathrm{HE}$ associated to a vibrational origin.

\subsubsection{Temperature Dependency}

In the peak analysis of the previous sections, every so often we came across spectra, which change with the cluster temperature. The alteration of the spectra can assume many different forms. Some sizes show additional peaks or shoulders while others show merely variations in the resulting VDEs, which may or may not be caused by different cluster temperatures. In order to get better understanding of the influence of the temperature on the spectra, we have recorded photoelectron spectra of the sizes 21 as well as 44 to 60 at various temperatures.

Let us start our analysis with the two prominent cases, where the temperature has an obvious impact on the spectral shape. The first case is the temperature dependency of the small pre-peak of the sizes 21, 26, and 53. For all three sizes peak II vanishes at temperatures some $10 \mathrm{~K}$ above $100 \mathrm{~K}$, e.g. for $\left(\mathrm{H}_{2} \mathrm{O}\right)_{21}{ }^{-}$it is barely detectable at $120 \mathrm{~K}$ (cf. fig. B.1. p. 155). Additionally there may be also changes in the line shape of the main peak, which will be discussed later.

The second case is the structural alteration of the main peak of $\left(\mathrm{H}_{2} \mathrm{O}\right)_{48}{ }^{-}$. Of all investigated cluster sizes, its spectrum is the one effected most by the cluster temperature. So it makes a good candidate to start a detailed analysis of the temperature dependency of the main peak. It should be emphasized, however, that it represents a special case and can therefore not be used to draw general conclusions about the temperature dependency of the PES of water clusters.

The spectra of $\left(\mathrm{H}_{2} \mathrm{O}\right)_{48}{ }^{-}$served already as an example for temperature dependency in the single GL fit analysis (cf. fig. 4.9). At that time we have found 3 temperature ranges(approximately $10 \mathrm{~K}-80 \mathrm{~K}-140 \mathrm{~K}-300 \mathrm{~K}$ ), where the line shape varies considerably. In neither range worked a single GL fit as a good approximation of the experimental data. Later it became clear that 2 or even 3 GL function would produce a much more accurate fit.

Fig. 4.26 shows the same spectra from the three temperature ranges fitted with 3 GL functions. Instead of the strongly shifting position of the peak maxima as in the case of the single fit, the temperature dependency is expressed now by different peak intensities, mainly of the peaks Ia and Ib. In the low and high temperature range peak $\mathrm{Ib}$ is the dominant one, whereas around $100 \mathrm{~K}$ Ia reaches the same intensity as Ib. Nevertheless, even in the 3 GL picture shift the VDEs slightly and the peak shape seems to be affected by the temperature too.

For a more quantitative analysis various peak parameters resulting from 2 GL and $3 \mathrm{GL}$ fits have been plotted versus the cluster temperature in fig. 4.27. If several measurements for one temperature exist, the mean value is plotted instead with error bars indicating the statistical standard deviation. The upper most panel shows the VDEs of peaks Ia and Ib. From $90 \mathrm{~K}$ to $130 \mathrm{~K}$ both shift to lower binding 

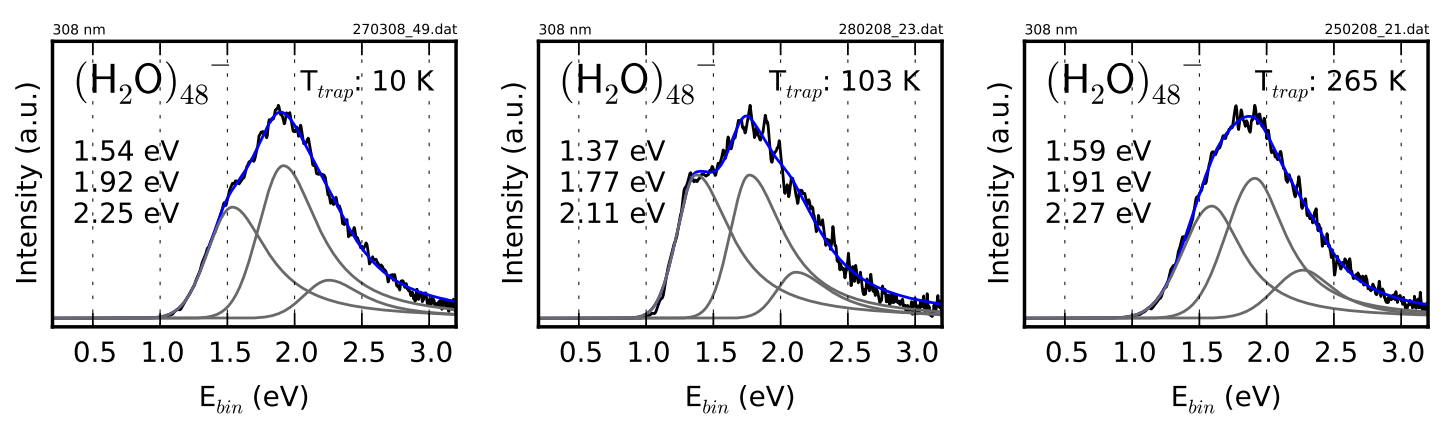

Figure 4.26.: Temperature dependent peak distribution of the photoelectron spectra of $\left(\mathrm{H}_{2} \mathrm{O}\right)_{48}{ }^{-}$using 3 GL fits.

energies. The maximum shift of about $100 \mathrm{meV}$ is reached near $110 \mathrm{~K}$. The next plot shows how the energy difference between both peaks, $\Delta E_{T}=\mathrm{VDE}(\mathrm{Ib})-\mathrm{VDE}$ (Ia), evolves in respect to the difference they have at $10 \mathrm{~K}$. It shows that the peaks move apart from each other by about $50 \mathrm{meV}$ at the temperature region around $110 \mathrm{~K}$ and move closer together $(50 \mathrm{meV})$ for temperatures beyond $140 \mathrm{~K}$. In the lowest of the three upper panels the proportional peak heights are plotted. Again the temperature region at $110 \mathrm{~K}$ stands out, because both peaks assume here the same intensity, while otherwise peak Ib is dominant. Both, the $2 \mathrm{GL}$ and $3 \mathrm{GL}$ analysis yield in general very similar values, larger deviations are visible predominantly for temperatures $>140 \mathrm{~K}$.

The undermost panel shows the evolution of the line shape parameter ( $f w h m$, $\left.\sigma_{G}, \sigma_{L}\right)$ with the temperature for the $2 \mathrm{GL}$ fit and the $3 \mathrm{GL}$ fit respectively. The Gaussian width parameter $\sigma_{G}$ is for all temperatures in very good agreement for both fit variants. $\sigma_{L}$ in contrast has the typical systematic shift of about $100 \mathrm{meV}$ when switching from 2 to $3 \mathrm{GL}$ functions, but also shows more variations with changing temperature. $\sigma_{G}$ decreases by up to $50 \mathrm{meV}$ or $25 \%$ in the temperature region near $110 \mathrm{~K}$. Depending on the evolution of $\sigma_{L}$, this means that either the line shape assumes a higher asymmetry ( $3 \mathrm{GL}$ fit: $\sigma_{L}$ stays almost constant) or the peak width decreases in that specific temperature region (2 GL fit: $\sigma_{L}$ decreases too).

Before we continue to analyze more cluster sizes, we should investigate how meaningful the numbers of the current analysis are in expressing a temperature dependency. Since the recording of a spectrum is a statistical process, the spectrum will always show small variation in its shape. This in turn causes the fit to yield slightly different parameters. The variation of the fit results caused by statistical variations depend also of the number of fit functions. In general, more fit functions result in a greater spread, since they can adapt easier to small details. This is particularly true, when the main peak has few substructure to "anchor" the individual GL functions. The task ahead is now to untangle the purely statistical variations from the 

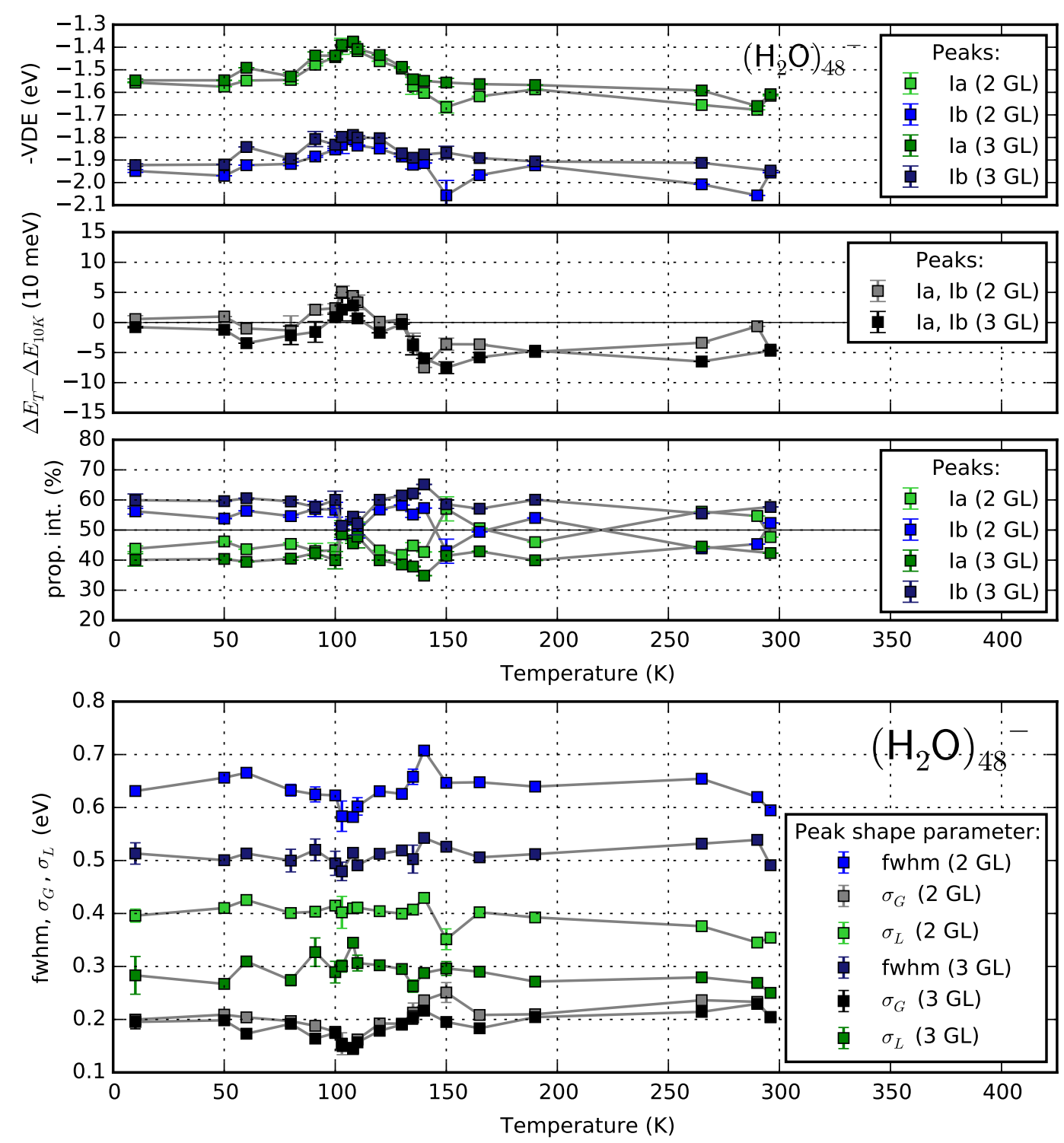

Figure 4.27.: Temperature dependent evolution of the VDEs (upper three plots) and peak parameters (lower plot) of $\left(\mathrm{H}_{2} \mathrm{O}\right)_{48}{ }^{-}$.

ones caused by the temperature.

The $\left(\mathrm{H}_{2} \mathrm{O}\right)_{48}{ }^{-}$temperature measurements provide enough data to get some rough estimates. Single temperature measurements at $103 \mathrm{~K}$ and $150 \mathrm{~K}$ show that the VDEs of peak Ia and Ib cover a range of $30 \mathrm{meV}$ to $70 \mathrm{meV}$ (3 GL). This corresponds to a standard deviation of about $20 \mathrm{meV}$. The VDE range per peak covered within one of the three temperature intervals $(10 \mathrm{~K}-80 \mathrm{~K}-160 \mathrm{~K}-300 \mathrm{~K})$ amounts to $100 \mathrm{meV}$ for both lower temperature intervals and $130 \mathrm{meV}$ for the high temperature interval respectively. When looking at all temperatures the VDEs spread 
from $200 \mathrm{meV}$ to $250 \mathrm{meV}$ for each peak. In the cases where averaging is applied, variations of the VDEs in a range of about $50 \mathrm{meV}$ may have a purely statistical origin and can not unambiguously attributed to temperature effects. However, if many small stepped temperature measurements exist, even smaller shifts can be identified, if the neighboring data points support a single trend. Other cluster sizes were measured only at few temperatures. In such a case averaging does not apply and we can only use the total spread as an estimate. From the data above follows that within a VDE range of about $100 \mathrm{meV}$ to $130 \mathrm{meV}$ a statistical or temperature related cause of the shift can not be distinguished.

For the relative shifts in peak distances $\left(\Delta E_{T}-\Delta_{10 \mathrm{~K}}\right)$ an analogous analysis yields that in the case of averaging fluctuations within a range of few $10 \mathrm{meV}$ can be attributed to a statistical origin. For sizes with only few temperature measurements, this range expands to roughly $100 \mathrm{meV}$.

The proportional peak heights can show large variations for some sizes. As a rough estimate, we attribute a height variation of $7 \%$ to a statistical origin in the case of many temperature measurements (and averaging) and a variation of up to $20 \%$, when there are only few data points.

As for the case of the peak shape parameter, peak analysis has already revealed that the high binding energy slope of the experimental spectra is less well defined than the low binding energy slope. The number of our measurements and their accuracy is therefore too low to obtain meaningful results from the temperature analysis of the $\sigma_{L}$ and consequently the full width. In contrast $\sigma_{G}$ has turned out to be much better defined, so that we believe its analysis to be meaningful within $20 \mathrm{meV}$, where higher statistics exist and $50 \mathrm{meV}$ for the sizes with few temperature measurements.

For all other cluster sizes is the impact of the temperature on the spectra much smaller than for $\left(\mathrm{H}_{2} \mathrm{O}\right)_{48}{ }^{-}$and any influence (if at all) is hence harder to detect. One general qualitative trend is, however, visible for almost all cluster sizes: any structures of the main peak like shoulders or dents in the slopes are more pronounced in the lower temperature range from $10 \mathrm{~K}$ to $140 \mathrm{~K}$. With higher temperatures $(>140 \mathrm{~K})$, the tip of the main peak often becomes broader and less pointed, so that it can be filled out less and less by a single peak. Fig. 4.28 shows few exemplary spectra in their more structured version at low temperatures (upper row) and the less structured version at high temperatures (lower row).

The broadening of the peak tip and declining structures causes the individual peaks of a multi GL fit to arrange themselves in a slightly different manner than for the cold clusters. Often the distance of the peak maxima decreases and the intensities become more equal. This leads to a tendency towards more symmetrical line shapes, i.e. to an increase in $\sigma_{G}$. On the other hand the broad, unstructured peak tip can cause larger fluctuations of the resulting VDEs, because without clear substructures are the fits more susceptible to small statistical variations of the experimental data. 

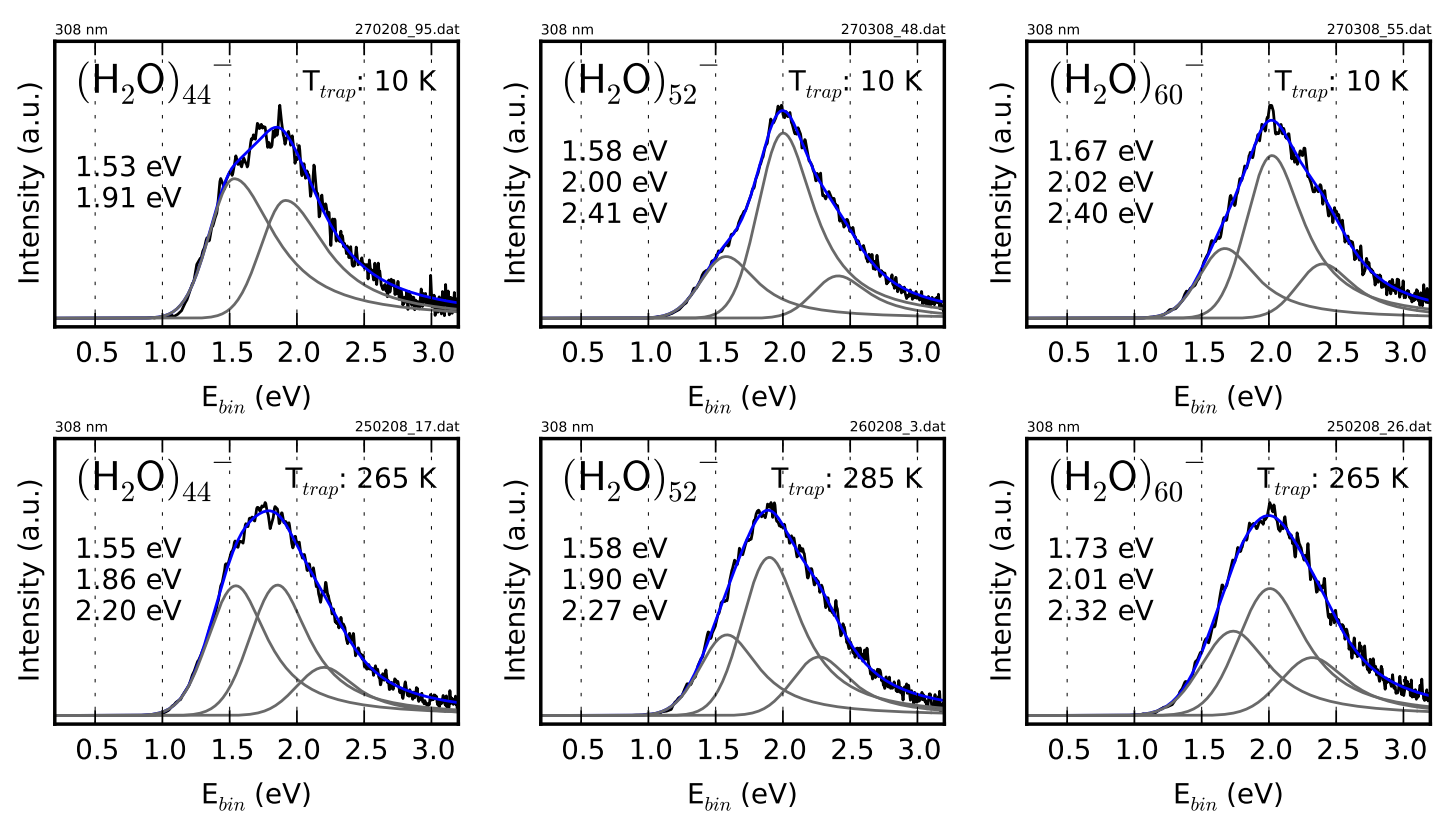

Figure 4.28.: Impact of the cluster temperature on the peak shape of photoelectron spectra. Upper row: more structured spectra at low temperatures. Lower row: less structured spectra with broader peak tips at high temperatures.

In the following we conduct the temperature analysis for sizes between 44 and 60 water molecules. The sizes around $\left(\mathrm{H}_{2} \mathrm{O}\right)_{48}{ }^{-}$are interesting due to its obvious temperature dependency. The size range 50 to 60 contains the magic sizes found in the mass spectrum. The plots can be found in chapter B in the appendix. Some of the spectra were measured at only few temperatures and only once or twice per temperature. This allows to determine only coarse trends, and single deviations at one temperature must be treated carefully.

$\left(\mathrm{H}_{2} \mathrm{O}\right)_{44}{ }^{-}$to $\left(\mathrm{H}_{2} \mathrm{O}\right)_{46}{ }^{-}$show no distinct trends for any of the investigated parameters (fig. B.2, B.3, B.4. p. 156 ff.). In the low temperature range $(<150 \mathrm{~K})$ the VDEs of the $2 \mathrm{GL}$ and 3 GL peak analysis result in approximately the same value while in the high temperature range the VDEs deviate more. The energy difference of the peak maxima appears to decrease slightly. The dominant peak depends in the high temperature range on the number of GL functions. So is peak Ia dominant for all temperatures (although with decreasing intensity) in the 2 GL analysis, whereas in the $3 \mathrm{GL}$ case peak Ib has the higher intensities in the high temperature range. $\sigma_{G}$ stays almost constant, but again the differences between 2 GL and 3 GL results are larger for higher temperatures.

The spectra of $\left(\mathrm{H}_{2} \mathrm{O}\right)_{47}{ }^{-}$show a similar temperature evolution (fig. B.5, p. 159 ) like $\left(\mathrm{H}_{2} \mathrm{O}\right)_{48}{ }^{-}$. Notable are the different results for the peak dominance for high 
temperatures from the 2 GL and 3 GL analysis respectively. The decrease of the peak distances complies with the general trend. The increase of $\sigma_{G}$ is, however, minimal.

The VDEs of $\left(\mathrm{H}_{2} \mathrm{O}\right)_{49}{ }^{-}$depend heavily on the number of fit functions for temperatures $>110 \mathrm{~K}$. This is caused by a shift of the dominant peak from Ib to Ia for rising temperatures (one might argue that the distribution switches rather from $\mathrm{Ia} / \mathrm{Ib}$ to $\mathrm{Ib} / \mathrm{HE}$ ). For the low temperature range up to $110 \mathrm{~K}$ the VDEs of the $3 \mathrm{GL}$ analysis follow the development of $\left(\mathrm{H}_{2} \mathrm{O}\right)_{48}{ }^{-}$. The high temperature range shows strong fluctuations, but has to few data points for a meaningful statement.

For the cluster sizes 50 and 51 there are only 2 GL fits resulting in a Ib/HE peak distribution. As the peak analysis has shown, the steep rise of the Gaussian slope makes the existence of a Ia peak very unlikely. The temperature analysis therefore uses the peaks Ib and HE instead (fig. B.7, B.8, 161 ff.). All parameters change only little with the temperature. For higher temperatures $(>150 \mathrm{~K})$ the inter-peak distance decreases slightly and the intensities approach each other. As $\sigma_{G}$ shows a slight increase too, these are all indications for the typical broadening of the peak tip at high temperatures.

For $\left(\mathrm{H}_{2} \mathrm{O}\right)_{52}{ }^{-}$the $2 \mathrm{GL}$ and $3 \mathrm{GL}$ peak analysis resulted in very similar values (fig. B.9, p. 163). The peak distance decreases distinctly for higher temperatures and also the approximation of the intensities follows the general trend. The $2 \mathrm{GL}$ fit at the two highest temperatures resulted in a $\mathrm{Ib} / \mathrm{HE}$ distribution rather than in the Ia/Ib. This causes the large shifts of the line shape parameter $\left(\sigma_{G}, \sigma_{L}\right)$ at that temperatures.

The temperature analysis of the sizes 53 to 59 (except 57, for which not enough temperatures dependent data is available) results in fairly stable parameters, although the sizes 53 to 56 partly exhibit large shift between single temperatures. Only the peak heights display a trend to equal their values. All in all better statistics would be required to determine, if some temperatures alter the spectra significantly.

The VDEs of $\left(\mathrm{H}_{2} \mathrm{O}\right)_{60}{ }^{-}$are fairly constant (fig. B.16, p. 170). The peak distances show the usual decrease with higher temperatures and the peak intensities approach each other. The 2 GL analysis resulted only at very low temperatures in a $\mathrm{Ia} / \mathrm{Ib}$ distribution. Higher temperatures show a $\mathrm{Ib} / \mathrm{HE}$ distribution, which causes a strong increase of $\sigma_{G}$ and subsequently a decrease of $\sigma_{L}$.

Apart from few size related temperature dependencies, most sizes show this trend towards a broad peak tip for high temperatures, which causes the inter-peak distance to decrease. In the case of $\left(\mathrm{H}_{2} \mathrm{O}\right)_{50}{ }^{-}$and $\left(\mathrm{H}_{2} \mathrm{O}\right)_{51}{ }^{-}$the temperature analysis has shown that this applies also to the peaks Ib and HE. As a last point it is interesting to investigate, if this trend is also applicable to the $\mathrm{Ib} / \mathrm{HE}$ peak relation of the other sizes. One should keep in mind though that the intensities of the HE peak are often much smaller than the Ib intensities (in respect to Ia). Together with the usually broader Lorentzian slope, this results in a stronger fluctuations of 
the HE peak maxima localization. We have investigated the shifts of the inter-peak distances for the size range 44 to 60 . In the low temperature range $(<130 \mathrm{~K})$ the shifts range almost always from $0 \mathrm{meV}$ to $50 \mathrm{meV}$. In the high temperature range the shifts vary greatly in their amount: some lie around $50 \mathrm{meV}$, others reach occasionally $100 \mathrm{meV}$ or more. The number of measurements and the accuracy of the maxima localization allow to detect only coarse trends. Although the peak distances at the highest temperature measurements are mostly smaller than the reference distance at $10 \mathrm{~K}$, a general trend could not be identified due to the strong fluctuations between individual temperatures.

\section{Discussion of the Temperature Dependency}

Does the photoelectron spectra of water clusters change with the temperature? The answer to this question is a clear affirmative, when considering the whole temperature range. For all spectra one finds changes in the peak shape, at least for the low and high temperature range. The boundary between the two ranges lies somewhere between $120 \mathrm{~K}$ and $150 \mathrm{~K}$. Some sizes, in particular 47, 48, and 52 exhibit a third temperature range from roughly $100 \mathrm{~K}$ to $140 \mathrm{~K}$, where the main peak changes its shape again, resulting in distinct shifts in the peak parameters. There is also a general trend to these changes: from a more structured, stronger pointed line shape to a less structured peak with a broader tip. The data of the proportional peak intensities and the peak distances for the particular temperature sensitive sizes $(47,48,52,60)$ indicate that the substructure is most distinct at temperatures near $100 \mathrm{~K}$.

The changes in the peak shape is reflected in the results of the peak analysis. Which in turn can greatly change with the number of fit functions used. Since the spectra show statistical variations by themselves, a certain fluctuation of the fit results is expected.

Changes in the photoelectron spectra are usually related to structural alteration of the cluster. Caloric measurements of water clusters [33, 34, 60] support this claim. In fig. 4.29 (a) the mean inner energy per molecule of an ensemble of $\left(\mathrm{H}_{2} \mathrm{O}\right)_{\mathrm{n}}{ }^{-}$clusters $(n=48,118)$ is plotted as a function of the heat bath temperature. The progression of the mean inner energy singles out three temperature regions. For $\left(\mathrm{H}_{2} \mathrm{O}\right)_{48}{ }^{-}$these are the low temperature range up to $93 \mathrm{~K}[33]$ or $97 \mathrm{~K}[60]$ respectively, where the cluster data follows closely the caloric curve of bulk ice. The second range is defined by a steep rise of the mean inner energy until the heat bath temperature reaches approximately $140 \mathrm{~K}$. Then the curve levels off and stays almost at the same energy, forming the third range.

As discussed in [33], the three temperature regions are related to three different structural states of the water cluster. At low temperatures the clusters are in the solid phase. The close resemblance of the caloric curve with bulk water ice even suggests a very similar vibrational density of states. The transition temperature 


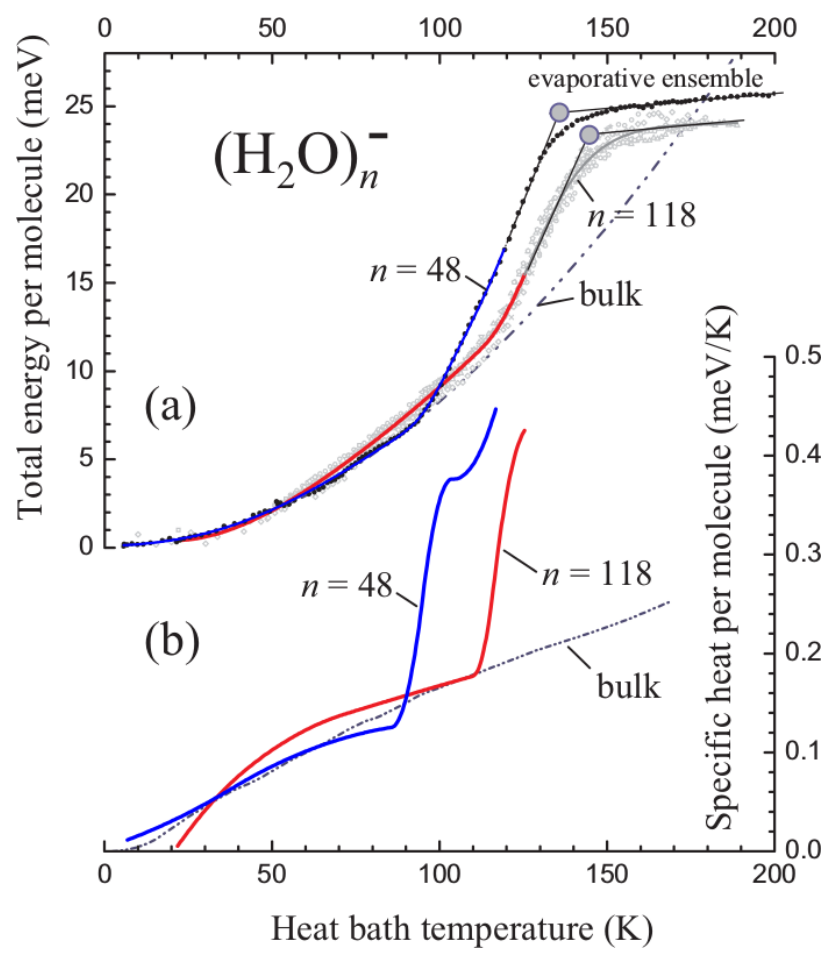

Figure 4.29.: Total energy (a) and specific heat (b) per molecule in dependency of the heat bath temperature for $\left(\mathrm{H}_{2} \mathrm{O}\right)_{48}{ }^{-}$and $\left(\mathrm{H}_{2} \mathrm{O}\right)_{118}{ }^{-}$(from [33]).

$T_{t r}$, at which the caloric curve sharply bends upwards, marks the onset of a gradual melting transition. In this temperature range the specific heat per molecules increases dramatically (fig. 4.29 (b)). At a certain temperature, $T_{\text {evap }}=136$ (6) $\mathrm{K}$ for $\left(\mathrm{H}_{2} \mathrm{O}\right)_{48}{ }^{-}$, the inner energy stops raising with the heat bath temperature and saturates at an energy $E_{\text {evap }}=24.8(10) \mathrm{meV}$. This marks the transition from the canonical ensemble to the evaporative ensemble[64]. The clusters are now in a state, where evaporative cooling is in equilibrium with heating by the heat bath. That means that the cluster temperature does not follow any longer the heat bath temperature, but stays at $T_{\text {evap }}$.

The transition and evaporative temperatures for $\left(\mathrm{H}_{2} \mathrm{O}\right)_{118}{ }^{-}$are slightly higher and amount to $T_{t r}=118(3) \mathrm{K}$ and $T_{\text {evap }}=144(6) \mathrm{K}$ respectively [33]. Measurements on more cluster sizes have confirmed that the transition temperature in general increases with increasing cluster size 60]. So does it vary in the size range from 48 to 58 between $94 \mathrm{~K}$ to $97 \mathrm{~K}$ and amounts to $106 \mathrm{~K}$ for $\left(\mathrm{H}_{2} \mathrm{O}\right)_{66}{ }^{-}$. For sizes $>200$ water molecules $T_{t r}$ seems to converge towards a value near $130 \mathrm{~K}$.

How do the caloric measurements relate to the temperature analysis of the photoelectron spectra? The three temperatures ranges found by the $\left(\mathrm{H}_{2} \mathrm{O}\right)_{48}{ }^{-}$analysis are in very good agreement with ranges marked by $T_{t r}$ and $T_{\text {evap }}$ of the caloric curve. The low temperature static range up to $80 \mathrm{~K}$ indicates the solid phase of 
the cluster. The equally static range starting at $140 \mathrm{~K}$ represents the "evaporative" phase. Judging from the caloric measurements one has to assume that the cluster temperature stays more or less constant at $T_{\text {evap }}$ even for trap temperatures $>140 \mathrm{~K}$. If this assumption is correct, then we must attribute all fluctuation of the peak parameters in this temperature range to a broader temperature distribution within the cluster ensemble or to purely statistical variation of the experimental data. Between $T_{t r}$ and $T_{\text {evap }}$ lies, according to the caloric measurements, the transitional phase, where at least partial melting of the cluster occurs [33]. It is also the range where the largest shifts of the VDEs are detectable, covering a range of $0.2 \mathrm{eV}$.

Two more sizes have been found, $\left(\mathrm{H}_{2} \mathrm{O}\right)_{47}{ }^{-}$and $\left(\mathrm{H}_{2} \mathrm{O}\right)_{52}{ }^{-}$, which exhibit three temperature ranges that can be correlated with the ranges from caloric measurements. However, additional sizes, like 49, 51, 53 and 60 may also show detectable phase transitions, if measured in more detail.

For most other investigated sizes there are at least a distinction of two temperature ranges possible. The transition temperature lies between $130 \mathrm{~K}$ and $160 \mathrm{~K}$. This correlates to the transition from the solid/transitional phase to the evaporative phase.

The temperature of the cluster has therefore clearly an impact on the photoelectron spectra, when considering a wide temperature range. The changes of the line shapes result in shifts of the peak maxima locations. These can either be small shifts, when the peak distribution remains similar, or rather large ones, when the peak distribution reverses (cf. 2 GL fits for $\left(\mathrm{H}_{2} \mathrm{O}\right)_{49}{ }^{-}$or $\left(\mathrm{H}_{2} \mathrm{O}\right)_{60}{ }^{-}$). Since we used measurements from all temperatures for the VDEs vs. $n^{-1 / 3}$ plots, it becomes clear now that the formation of VDE "bands" for each peak is largely caused by the temperature dependency of the spectra. Since the VDE shifts originating from the temperature move systematically in one direction, they may also impact the extrapolation to the bulk value. In the previous chapters we have found that an extrapolation to the bulk is meaningful only, when including sizes larger 80 . Since the majority of the relevant cluster sizes were recorded at temperatures of $80 \mathrm{~K}$ or lower, a large deviation from previous fits is thus not to be expected. Nevertheless, to get an estimate of the influence of temperature on the fit results, we have redone the fit (3 GL) including only measurements between $10 \mathrm{~K}$ and $85 \mathrm{~K}$. It yielded a bulk value of $3.59(2) \mathrm{eV}$ compared to $3.60(2) \mathrm{eV}$.

\subsection{Discussion}

The peak analysis of the previous sections has revealed that the photoelectron spectra of water clusters are more complex than a single, broad, and slightly asymmetric peak, which they seem to be on a first glance. There are those few sizes $(21,26$ and 53) which show a small additional peak in front of the main peak, which can only 
be mapped by an additional fit function. But even the main peak is often badly fitted by a single GL function, especially for sizes between 30 and 65 . This resulted in a spread of the VDEs of a single size, because the peak shape was found not only to change between different sizes, but also between different temperatures. The analysis of the line shape parameters resulting from the single fit approach showed a strong variation of the peak shape, which is at least partially caused by the bad agreement of the fit with the experimental data. In short, the reproduction of the spectral features by a single GL fit proved to be adequate only for sizes consisting of more than 70 or 80 water molecules, while for smaller sizes it merely yields "averaged" peak parameter.

To improve the agreement of the fit and to reduce some of the shortcomings of the single GL fit analysis, the main peak of spectra has been fitted with 2 GL functions plus an additional function for sizes with an existent pre-peak. This improved the agreement of the fit with experimental data enormously. The large shifts in the spectral features in the size range 30 to 65 could now be understood as changes in intensities of two peaks (Ia and Ib). The intensities follow a constant growth pattern with the cluster size. Peak Ia is the dominant peak at small sizes. Peak Ib starts to grow in intensity in the mid 30s, gains equal intensity with Ia around 46 and becomes the dominant peak for all larger sizes. Starting at size 52 the 2 GL approach becomes ambiguous in the sense that it is often possible to fit the experimental data in two variants. Variant 1 yields a Ia/Ib peak distribution, whereas variant 2 results in a Ib/HE peak distribution. For both variants is Ib the dominant peak and both yield quite similar VDEs. When comparing the line shape parameters, however, the variants result in huge differences. While variant 1 shows an almost monotonic increase of the $\sigma_{G}$ and $\sigma_{L}$, variant 2 exhibits discontinuities when switching from the Ia/Ib distribution of the smaller sizes $(<50)$ to the $\mathrm{Ib} / \mathrm{HE}$ distribution starting at 50. Unfortunately is the qualitative agreement of the fit better for variant 2. Further disagreement can result from the temperature dependency of the spectra: some sizes switch between the two fit variants for rising temperatures.

Besides this discrepancies there are spectra in the 50 to 60 range exhibiting features which suggest the presents of an additional peak. Fitting those sizes with 3 GL functions further improves the agreement of the fit with the experimental data in the size range from approximately 40 to 70 . It also results in an unambiguous way to fit the data in contrast to two fit variants of the $2 \mathrm{GL}$ analysis. What remains are the discontinuities in the line shape parameter stemming from a change of the number of GL functions. There is no solution to that problem besides extending the size range of the 3 GL fit far beyond sizes, whose spectral features indicate the presence of 3 peaks.

Neither of the discussed approaches seems to be able to deliver a consistent analysis of the photoelectron spectra of water clusters. This leads to question, if one or multiple GL functions are adequate fit functions for the problem at hand. So let's 
recapitulate the origin of the GL function. The GL was chosen as a simple, analytic function to approximate experimental spectra and help to determine the location of the peak maximum, some line shape parameter and to separate isomers. The justification was a good empirical agreement with experimental photoabsorption as well as the photoemission spectra of water clusters and bulk water (absorption only) [3, 16, 39, 76]. The GL function describes therefore in the first place the envelope of the spectra ignoring a possible substructure. Most earlier measurements, however, either do not show a significant substructure of the main peak 41, 77] or could not resolve it[16]. Only Coe et al. found a shoulder on the high binding energy slope for sizes consisting of up to 30 water molecules [17].

The question, if a GL function also adequately represents the individual substructures and thereby makes their maxima determinable, ultimately leads to the question of the origin of those substructures identifiable in our spectra. In the case of the few sizes exhibiting pre-peaks seems the answer to be straightforward: the pre-peaks represent another cluster isomer with an electron binding motif resulting in a lower binding energy. The number of fit function for the main peak has virtually no influence on the peak parameter of the pre-peak, so they seem to be decoupled enough to be treated by two (or more) GL functions. What does this imply for the GL functions comprising the main peak? Both fit variants, 2 GL and $3 \mathrm{GL}$, showed that the peaks Ia and Ib are dominant in different size ranges with a transition region in between. The dominant peak must be interpreted as an isomer, which would make peak Ia and Ib contributions from different water isomers, at least in those size ranges. However, it is more difficult to say, if peak Ib always represents an isomer or only starts to be one at a certain cluster size. The peak analysis showed that Ib starts to increase in intensity relative to peak Ia in the mid 30s size range. This may indicate that the cluster beam composition changes and contains an growing share of another isomer. Coe et al. attributed this peak to vibrations for cluster sizes $\leq 30[17$. It was assigned to the $\mathrm{O}-\mathrm{H}$ stretch vibration of water due to its energetic distance to peak Ia, which is reduced by a factor of $\sqrt{2}$ upon deuteration. The peak distance ratios of the $2 \mathrm{GL}$ analysis(fig. 4.21) support this assignment and indicate that starting at size 33 peak Ib originates mainly from an isomer, whereas for smaller sizes it might have a vibrational cause. However, the same analysis for the 3 GL approach locates the transition at larger sizes starting around 43 (cf. chapter 5.2.2, fig. 5.5).

The high binding energy peak $\mathrm{HE}$, visible in the $3 \mathrm{GL}$ analysis and the second variant of the 2 GL analysis, does not show an increasing intensity with growing cluster size. Our data shows no indication that the HE peak will gain an intensity comparable with peak Ib at very large cluster sizes. Therefore we do not assign peak HE to another isomer, but also propose a vibrational origin.

To summarize the current results, we have found indications in the experimental spectra of a substructure consisting of up to four distinct peaks. The number and distribution of peaks depends heavily on the size of the clusters and to a certain 
degree on the temperature. With the exception of the high binding energy peak, we assign the peaks to distinct isomer classes. Each class is formed by clusters with similar solvation potentials of the excess electron, which refer to similar solvation structures. As to where the hydrated electron is localized, can not be determined directly from the photoelectron spectra. It is, however, possible to make some deductions for the very small and very large clusters.

The very small clusters must start with a surface localized electron because they just consist of surface molecules. So there must be a minimum number of water molecules required to form the surroundings for the electron internalization. Therefore we can assume that isomer Ia represents a isomer class with surface localized excess electron, at least for the small cluster sizes. Since we have found several changes in the slope of the size dependent VDE evolution, indicating structural rearrangements, it is not clear, up to which size this assignment stays valid. We try to settle this point in sec. 4.3 .2 in combination with theoretical calculations.

As for the very large clusters, we have found that the isomer Ib class is the dominant isomer species. No higher binding energy isomers of significant intensity has been detected. Isomer Ib exhibits a constant structural growth scheme for sizes larger than 80, which makes an extrapolation beyond the measured $\left(\mathrm{H}_{2} \mathrm{O}\right)_{1100}{ }^{-}$very plausible. So if we assume that an internal electron localization is the preferred solvation scheme for large systems, than we can assign isomer Ib clusters starting with size 80 to structures with internal electron localization. Based on the linear fits to the results of the single and multi GL analysis as well as the temperature analysis, we assign the extrapolated bulk value of isomer $\mathrm{Ib}$ to $3.6 \mathrm{eV}$ with an accuracy of $\pm 0.03 \mathrm{eV}$.

The intermediate size range shows many indications for structural changes. They cumulate in the region between 50 and 60, where almost every single size seems to undergo some rearrangement. Based on the experimental data only, it is therefore uncertain, how far we may extend the assumption of the large and small clusters into this size region. An assignment of isomer Ia to a surface isomer and isomer Ib to an interior isomer over the whole size range, can thus be only preliminary.

\subsubsection{Comparison with external experimental data}

How do our results compare with published data of other groups? The upper panel of fig. 4.30 combines the VDEs of our peak analysis (plotted faded in the background) with data of several groups published in recent years.

Neumark and coworkers published VDEs of three distinct water isomers (I, II, III) [77], labeled with (Neumark) in the legend of the plot. Isomer I confirmed the VDEs of the very early measurements[16] (hence isomer I). Isomer II was found in water and deuterated clusters, whereas isomer III was only detectable in PES of deuterated water clusters. In a later experiment the Argon for co-expansion in the cluster source was replaced by Neon at higher pressures [82. Under such source con- 


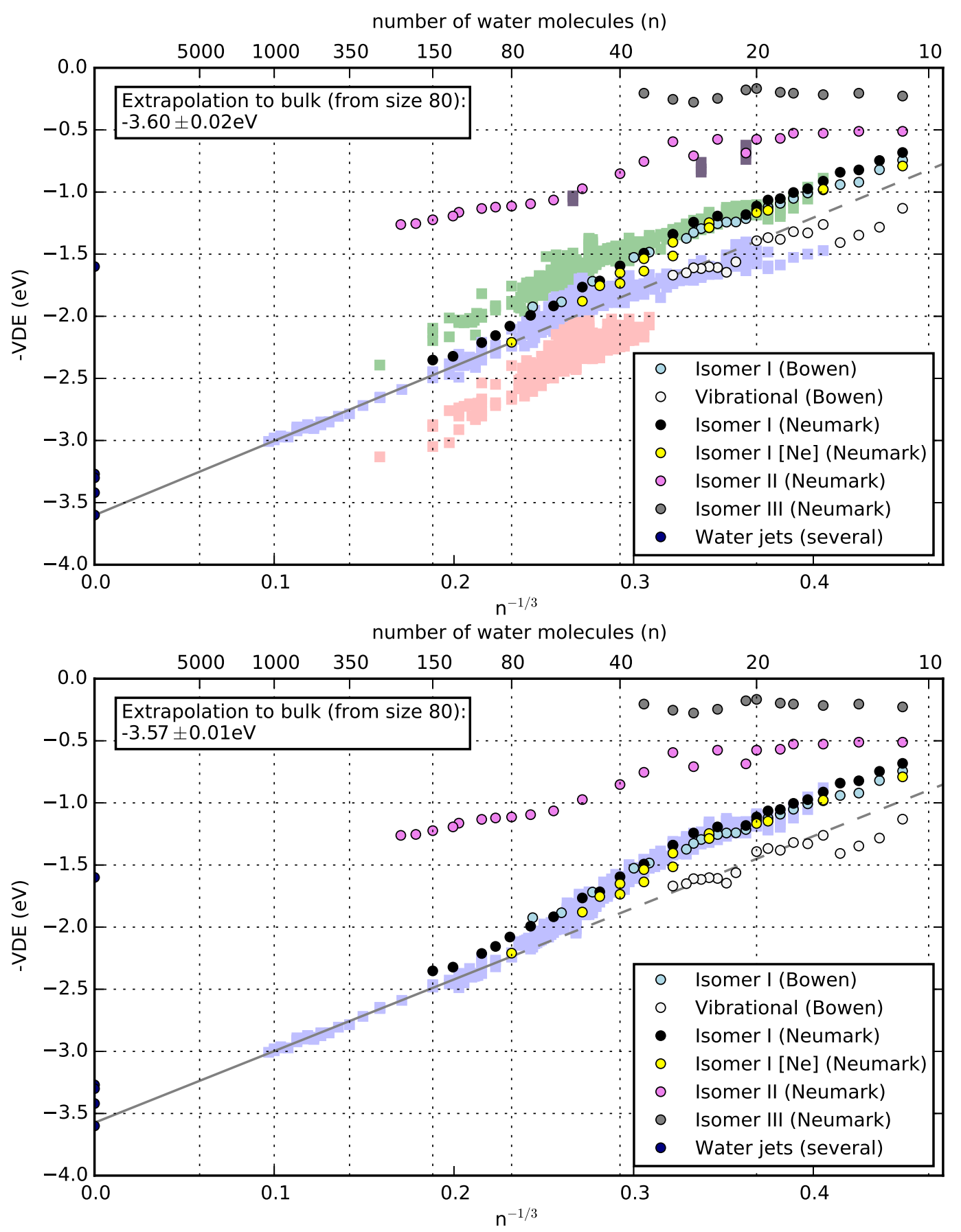

Figure 4.30.: VDEs of $\left(\mathrm{H}_{2} \mathrm{O}\right)_{\mathrm{n}}{ }^{-}$and water-jets published by other groups in comparison with the results of the multi GL analysis (upper panel) and single GL analysis (lower panel). 
ditions only isomer I clusters were detected in the photoelectron spectrum, which showed a slightly shifted VDE (additionally labeled with [Ne]) in respect to the earlier Ar measurements. The missing low binding energy isomers and a decrease in the internal conversion lifetime of excited water clusters was read as an indication that those clusters are vibrationally warmer than the ones produced at lower pressure Ar.

Experiments of the Bowen group could reproduce the findings of three isomers[17]. The VDEs of isomer I for sizes up to 69 water molecules are included in the plot and labeled with (Bowen). The peak analysis showed another peak at higher binding energy, which was attributed to $\mathrm{O}-\mathrm{H}$ stretch vibration.

Only recently several groups succeeded in measuring the kinetic energies of photoelectrons ejected from liquid water-jets, which allowed them to determine the VDE of the solvated electron in bulk water. The VDEs range from $3.27 \mathrm{eV}$ to $3.6 \mathrm{eV}[35,53,66,68,71]$. Additional a lower binding energy value was found at $1.6 \mathrm{eV}[68$.

The few sizes of our data exhibiting isomer II peaks are in good agreement with the more extended isomer II data of the Neumark and Bowen groups. The isomer I VDEs are in good agreement with our isomer Ia VDEs for small sizes up to approximately 30 water molecules. In the size range between 30 to 50 the VDEs lay in between our Ia and Ib VDEs. From 50 onwards they approach our Ib VDEs, but are slightly shifted to lower binding energies. The VDEs of the warmer cluster measurements of the Neumark group ([Ne]) are in better agreement with our data in this size range than the colder ones. The VDEs of the vibrational peak of the measurements by the Bowen group are in good agreement with our peak Ib VDEs for sizes $\leq 30$. For smaller sizes from 15 to 19 are our VDEs shifted by about $100 \mathrm{meV}$ to higher binding energies. That way they connect however nicely to the VDEs the Bowen group measured for sizes $<15$. The extrapolation of our isomer Ib data to a bulk value of $3.60(3) \mathrm{eV}$ conform with the experimental water-jet result of $3.6(1) \mathrm{eV}$ by the Neumark group [66]. The next nearest value of 3.46(6) eV [35], measured by Horio et al., is still considerably lower.

Given the excellent agreement of the VDEs for small cluster sizes $(<30)$ with isomer Ia VDEs and the fairly good agreement for larger sizes $>50$ with isomer Ib VDEs, it remains to find an explanation for the deviations in the transitional size range between 30 and 50. The lower panel of fig. 4.30 shows the same data sets in respect to the single fit analysis of our data. In this comparison the agreement of the VDEs is excellent for almost the whole size range. Only the large sizes $(>70)$ show a small shift to higher binding energies. However, the small shift found in the VDEs of the higher temperature measurements of the Neumark group[82] ([Ne]) compensates this discrepancy.

Since the single fit analysis yields weighted average VDEs of isomers Ia and Ib, the good agreement with the external isomer I data indicates that those measurements also represent an average value. In order to further investigate this point, we 
can compare available peak shape parameters. Fig. 4.31 brings the parameters published by the Bowen group [17] in relation to our data gained from the single fit analysis. In general is the agreement for all parameters quite good. $\sigma_{G}$ shows an

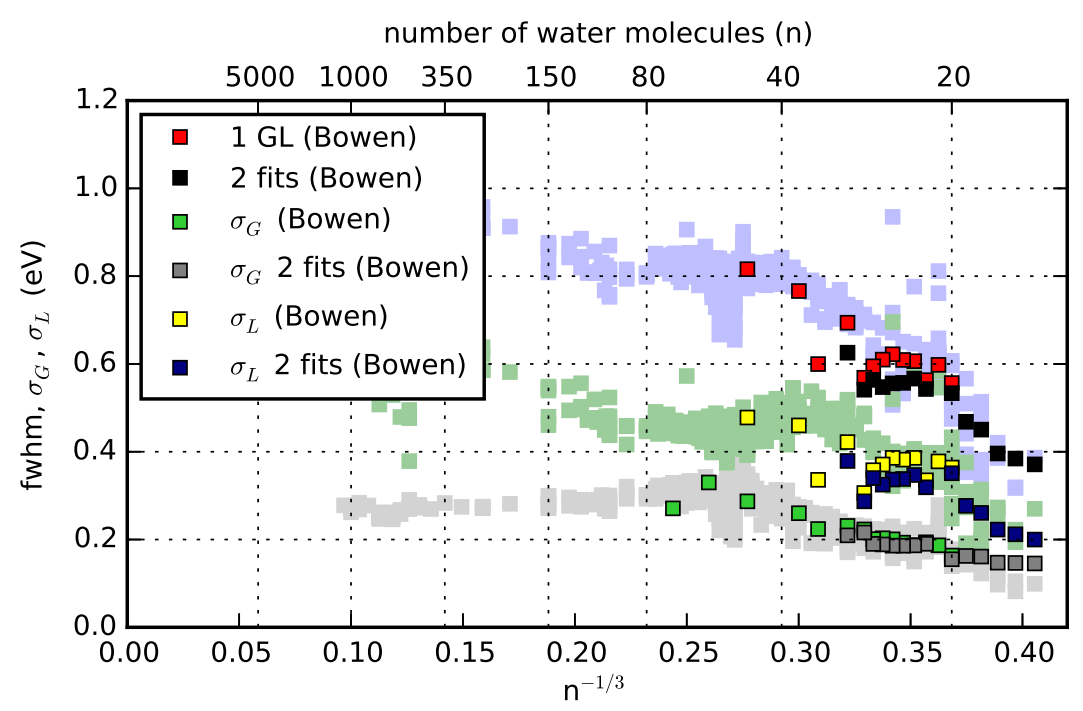

Figure 4.31.: Peak shape parameters published by other groups in comparison with the single GL fit results.

excellent agreement starting from size 19. Especially the maximum near size 50 is reproduced. For the small cluster sizes $(<19)$ our $\sigma_{G}$ is considerably smaller. The evolution of $\sigma_{L}$ in Bowens data does not show the maximum we find near 38, but the general trend is in accordance: a plateau between 20 and 27 and increasing values from 30 onwards.

The similarities in the development of the peak shape parameters suggests that the origin of the substructures visible in our spectra are also largely determine the peak shape of the spectra measured by Bowen and coworkers, but were not resolved for some reason. There is no peak shape data for the Neumark groups measurements available, however, the good agreement of the VDEs with Bowens data and the similar experimental setup (especially concerning the cluster source), makes it very plausible that their peak shapes are alike. If that is true, than contain all experimental spectra, at least to a certain degree, contributions from the two isomers Ia and Ib, which we identified in our spectra.

Where then originate the differences in the experimental spectra? The temperature analysis has shown that the temperature of the clusters and thus the temperature distribution of the cluster ensemble influences the peak shape. Our experimental setup is in this respect significantly different from the setups used by the Bowen and Neumark group respectively. Both groups use supersonic expansion sources to produce water clusters. The temperature is to a certain degree 
adjustable by the pressure of the gas used in co-expansion [82]. The Neumark group has reported on several occasions, how the carrier gas pressure affects the isomer distribution of isomers I, II and III in the cluster beam[77, 82]. The cluster beam is intersected by a pulsed electron beam, which causes the formation of cations and anions by collisional detachment and secondary electron attachment respectively [1]. The ionization processes, which take place after cluster formation, and especially the internalization of the excess electron, heat the clusters up. This is partially compensated by heat removal through the carrier gas. The reported presence of isomer II and III clusters under high pressure source conditions, shows that the clusters are indeed very cold. The temperature analysis of the sizes exhibiting isomer II in our data has shown that temperatures below $100 \mathrm{~K}$ are necessary to stabilize those isomers. Nevertheless, the missing substructures of the isomer I peak may indicate that the cluster beam has a broader temperature distribution and contains also higher temperature clusters, possibly because the heat transfer to the carrier gas is only partial. Another (or additional) explanation could be incomplete annealing: the low temperatures at cluster production may not be high enough to overcome the many metastable isomers produced by the source. The peak shape analysis of $\left(\mathrm{H}_{2} \mathrm{O}\right)_{48}{ }^{-}$and $\left(\mathrm{D}_{2} \mathrm{O}\right)_{48}{ }^{-}$provides hints for this: $\sigma_{G}$ assumes the smallest value at a temperature right above the temperature marking the melting transition. Since $\sigma_{G}$ seems to be a measure of the structure distribution within an isomer class, this indicates that special temperature conditions are necessary to gain a narrow isomer distribution.

In contrast is the cluster production and ionization in our experimental setup decoupled from the thermalization. Especially the transit region between source and trap provides for an additional annealing step before thermalization. The temperature distribution of the clusters can be assumed as very narrow, at least for temperatures below the evaporative ensemble. We consider the at least partial annealing and the narrow temperature distribution of the clusters as the main reason for a narrow structure distribution (or a small $\sigma_{G}$ in terms of peak shape parameter), which makes it possible to resolve the substructures of the isomer I peak in the photoelectron spectra.

\section{Experimental Peak Shapes}

As we have seen, the spectral line shape is an important observable, which depends strongly on the type of peak analysis. Extrapolations of width parameters must therefore be treated very carefully. Coe et al. calculated an extrapolated fwhm of about $1.04 \mathrm{eV}$ for the bulk[18]. This result is of the same order of magnitude as the widths yielded from water-jet experiments: $0.8 \mathrm{eV}$ (Siefermann et al. 68]), $1.1 \mathrm{eV}$ (Buchner et al.[13]), 0.84(5) eV (Horio et al.[35]) and 1.0(2) eV (Shreve et al. 66]). The extrapolated width is, however, based on an extrapolated $\sigma_{G}=0.55(2) \mathrm{eV}$ and assumes a constant $\sigma_{L}=0.49 \mathrm{eV}$ for all sizes with $n>60$. The current peak 
analysis of larger water clusters show that an extrapolation for sizes $<80$ must yield misleading results. Independent of the method of the peak analysis, it suggests that $\sigma_{G}$ stays well beneath $0.4 \mathrm{eV}$, while $\sigma_{L}$ contributes more and more to the fwhm value.

It should be noted that the asymmetric line shape evident in the cluster PES, is not always found for spectra from water jets. While the results of Shreve et al. clearly exhibit an asymmetry, Horio et al. did not find one and assume a full Gaussian line shape resulting in $\sigma_{G} \approx 0.37 \mathrm{eV}$.

An asymmetry of the line shape is more evident in photoabsorption measurements of the hydrated electron in bulk water [39, 76]. Since fitting the line shape with a Gaussian-Lorentzian function originates from the similarities of these spectra, it is certainly interesting to look at the numbers. Two photoabsorption studies investigating line shapes in a temperature range from $270 \mathrm{~K}$ to $380 \mathrm{~K}$ yielded line shape parameters $\sigma_{G} \approx 0.3 \mathrm{eV}$ and $\sigma_{L} \approx 0.5 \mathrm{eV}$ at $274 \mathrm{~K}$, which correlate to $f w h m \approx 0.85 \mathrm{eV}$. The absorption maximum was found at $E_{\max } \approx 1.8 \mathrm{eV}$. They also found a weak temperature dependency, which causes $E_{\max }$ to shift to higher values for decreasing temperatures, whereas the width becomes narrower.

Besides the location of $E_{\max }$, the peak parameters resemble astonishingly well the peak parameters from the single GL analysis for cluster sizes consisting of more than 150 water molecules. A general resemblance of the peak shape has also been found for absorption spectra of water clusters 3 .

The origin of the asymmetric line shape of the absorption spectra is not completely understood by theoretical models. Most assume that one or more $s-p$ transitions compose the main peak of the bulk spectrum. The high binding energy tail is then formed by either an asymmetric remainder stemming from bound-tofree transitions (while the $s-p$ transitions are modeled by Gaussians) [38] or results from asymmetrically shaped single transition contributions, when summed up [65].

Since the absorption and photoelectron spectra originate from different transitions, it is questionable, if the peak shape parameters may be compared directly. The resemblance of the spectra suggest, however, that the single contributions of the underlying substructure are for both, absorption and photoelectron spectra, very similar.

\section{The Gaussian-Lorentzian as Fit Function}

The results of the peak analysis of experimental photoelectron spectra of water cluster anions rest on the validity of the assumption that the GL function is an adequate approximation of the overall spectrum or, as in our case, of the spectra of its individual contributors. The justification for using GL functions to fit the spectra, originates in all cases primarily from an empirically good agreement between the fits and the experimental data. Since we have only a basic, qualitative understanding of the general shape of the spectra and how it might be comprised 
(cf. chap. 3), this seems to be the best approach currently available. Nevertheless, for a better assessment of the results, it is import to be aware of the approximations we make by the GL peak analysis.

A single GL function shows an excellent agreement with non-structured spectra like bulk absorption spectra or large clusters PES, but fails for more structured data. This indicates that the overall spectrum is in good agreement, as long as many different contributors form the spectrum. With fewer contributors, a single GL fit represents more a weighted average, which is good at removing experimental noise, but also skips the details. The multi GL approach assumes that individual substructures can also be mapped by GL functions. As in case of the single GL fit, motivates ultimately a good agreement of the sum function with the experimental spectra this approach.

Since the indicators for possible substructures in the experimental spectra are primarily shoulders and their number is a priori unknown, it is impossible to determine the exact shape of the individual contributions directly by the fit. This means we have to make another approximation by assuming identical width parameters $\sigma_{G}$ and $\sigma_{L}$ for all GL functions. In the picture, where $\sigma_{G}$ represents mainly the structure distribution of a single isomer class and $\sigma_{L}$ the vibrational patterns of the structures of that class, this would mean that the different isomer classes possess the same structure distribution and vibrational behavior, which is not very likely. If the structures differ enough to yield distinct VDEs, then one can expect their vibrational excitation patterns to be different too. Some of the inconsistencies we have found so far may arise from that approximation: e.g. the discontinuities of the width parameters may be reduced, if they could evolve independently for each isomer class.

\section{Magic Sizes}

Water cluster anions in the size range from 50 to 60 show strikingly high fluctuations of the VDEs in the photoelectron spectra as well as abundances in the mass spectra for each additional water molecule. The changes of the VDEs has been found to be independent of the peak analysis method and concern all isomers of a size equally. This excludes the possibility that the shifts of the VDEs are merely results of a change in the isomer distribution. They are therefore deemed to be linked to structural changes of the clusters. Interestingly does the shape of the photoelectron spectra not change with each additional water molecule, but rather every two sizes. This means that apart from the location of the peak maxima, the spectra of the sizes 50 and 51,52 and 53,54 and 55, as well as 56 and larger, are qualitatively similar to each other, but differ considerably from their neighboring pairs.

The most pronounced differences in abundance of the mass spectra appear at a temperature around $120 \mathrm{~K}$. This coincides with the temperature interval bracketed by the melting temperature near $90 \mathrm{~K}$ and the evaporative ensemble temperature 
at about $140 \mathrm{~K}$. At these temperatures, structures with lower binding energy can evaporate molecules on the timescale of the experiment, while slightly more stable structures keep their original number of molecules.

The cluster sizes with enhanced stability, 50, 52, 54, 56, and maybe 58, are in contrast to measurements on cold water cluster anions, which were ionized by low energy electron attachment [45] and protonated water clusters $\left(\mathrm{H}_{2} \mathrm{O}\right)_{\mathrm{n}} \mathrm{H}^{+}[52$, which found higher stability for the sizes $51,53,55,57$. An possible explanation of this apparent discrepancy has been discussed already in [54]. In short, the neutral cluster sizes $51,53,55$, and 57 form structures with enhanced stability, which remain basically unperturbed by low energy electron attachment at low temperatures or by the addition of a proton. In the case of our water cluster anions, the excess electron interacts more strongly with the clusters. If the electron replaces one water molecule in the cluster, while keeping its structure intact, the anions with $n-1$ water molecules would be structurally very similar to the neutral clusters comprised of $n$ $\mathrm{H}_{2} \mathrm{O}$. An enhanced stability of the sizes 51,53 , and 55 of neutral clusters has also been found in a theoretical study[42], performing detailed structural calculations of water clusters.

\subsubsection{Comparison with theoretical data}

The question of the localization of the hydrated electron and its interaction with the solvent can only be answered in conjunction with theoretical calculations and models. A large number of model calculations are available now with all levels of approximations. The complexity of the problem is reflected in the strongly fluctuating results for the VDEs of water clusters. What is more, the assignment of the VDEs to a localization type (e.g. internal, surface, intermediate, etc.) shows considerable deviations and even contradictions. The calculated VDEs can be sorted into two classes: depending on the underlying model, sometimes only the relative changes in VDEs between cluster size can be determined with some accuracy, that is the slope in the VDE vs. $n^{-1 / 3}$ development. More sophisticated models may also allow to determine the absolute values of the VDEs.

The first results of a larger size range of calculated water VDEs originate from molecular dynamics (MD) simulations [5] conducted by Barnett et al. They provided also the first hint that one might find two distinct isomers species in experimental cluster beams, one for surface and one for interior localized excess electrons. In the same publication, the interior state VDEs of the two largest cluster sizes, 64 and 128, have been found in good agreement with the dielectric sphere model. A comparison with experimental data[77] showed later that the VDEs of the calculated surface isomer fell in the range of the highest experimentally determined VDEs, while the calculated interior state VDEs resulted in even higher values. Until now no such high VDEs have been found in experimental spectra. The conclusion at the time was that the model assumed a too strong electron-water interaction 
and that the VDEs must consequently be scaled by $60 \%[77$. Since scaling by $60 \%$ resulted in a good agreement of the calculated VDEs with the experimental data, at least the relative change of the VDE with the cluster size can be considered as valid.

As a first step we can therefore compare the relative change of calculated VDEs, that is the slope in a VDE vs. $n^{-1 / 3}$ plot, with the extrapolation from our peak analysis. We will consider only sizes $\geq 80$, because the evolution of the experimental VDEs showed a linear progression only starting from size 80 (the value of the slope for the experimental data stems from a fit starting at size 80). Since the available data for calculated VDEs is quite scarce, the determined slope values should be understood as an estimate for the order of magnitude. Table 4.1 summarizes the slope and intercept of the extrapolations to our data and calculations extracted from various theoretical publications. The intercept corresponds to the extrapolated VDE of the bulk.

\begin{tabular}{llll} 
slope $(\mathrm{eV})$ & intercept $(\mathrm{eV})$ & isomer/localization & source \\
\hline 5.8 & 3.57 & isomer Ib & single GL analysis \\
6.0 & 3.60 & isomer Ib & multi GL analysis \\
6.8 & 4.9 & interior & Landman[5] \\
6.5 & 3.6 & interior & Landman[6] \\
5.0 & 3.5 & cavity init. & Herbert[36] \\
6.9 & 3.7 & surface (TB) & Turi[73] \\
14.3 & 5.0 & interior (TB) & Turi[73] \\
8.8 & 5.2 & surface (LGS) & Turi[73] \\
13.4 & 6.2 & interior (LGS) & Turi[73]
\end{tabular}

Table 4.1.: Comparison of the slope and intercept values resulting from a linear fit to the VDEs of various sources.

The first four slopes from theoretical data are within $\pm 1 \mathrm{eV}$ in good agreement with our experimental slope values, which amount to approximately $6.0 \mathrm{eV}$. They all concern interior localized VDEs. The next one, labeled with (surface (TB)) and based on data from Turi, was, however, assigned to a surface state. In this very recent publication [73], Turi calculated the VDEs of many water cluster anions up to size $n=1000$. The initial cluster configurations were produced by performing molecular dynamics (MD) simulations using two different pseudopotentials, the Turi-Borgis (TB) and the Larsen-Glover-Schwartz (LGS) potential, because they produce a significantly different solvation structure for the excess electron [73]. Since this are the first calculations providing a large number of VDEs even for huge cluster sizes and due to strong deviation from our data we include also data for surface states 1 . Of all VDEs only the TB surface state calculation yielded a comparable

\footnotetext{
${ }^{1}$ The publication contains also data for non-cavity interior states. The resulting VDEs deviate,
} 
size dependency.

When looking at the intercepts or the VDE bulk values, the same calculations which yielded a fitting slope, also show a very good agreement $( \pm 0.1 \mathrm{eV})$ with our experimental data $(3.6 \mathrm{eV})$ (with the sole exception of the early Landman paper [5]). By contrast are the intercept values for the interior (TB) and both LGS calculation far too high.

With both, the slope and the intercept in good agreement for many calculations, the VDEs themselves can also be expected to coincide well with the experimental data. In fig. 4.32 the individual data points of the calculations are plotted overlaying our experimental data ${ }^{2}$. The upper panel uses as reference the multi GL analysis, the lower panel the single GL analysis.

The theoretical results from the Landman group were gained from first-principles simulations based on spin-density-functional theory, with all the valence electrons (that is, 8 electrons per water molecule) and excess electrons treated quantum mechanically [6]. They found three isomers, interior, surface, and diffuse, based on the localization of the excess electron. An exception form the small cluster sizes 15 and 30, where the initially prepared interior state converged into surface state. The lowest VDEs belong to diffuse state isomers, where the excess electron wave function is spread over a wide surface area. The calculated VDEs coincide well with experimental isomer II band.

The next higher VDEs stem from the surface isomer, for which the electron wave function is stronger localized than for the diffuse state. Most VDEs are in good agreement with the experimental isomer Ia, except for size 53, which showed an even higher VDE than the calculated interior state. The VDEs of the large sizes, 85 and 105, are to low, when comparing to the single fit analysis, but fit with the isomer Ia development from the multi GL analysis.

The interior states VDEs fit best to isomer Ib, although they are shifted in direction of lower binding energies.

Jacobson and Herbert used mixed quantum/classical molecular dynamics based on a one-electron pseudopotential model that incorporates many-body polarization[36]. The authors claim a prediction accuracy of the calculated VDEs of about $0.1 \mathrm{eV}$. They have found four distinct isomer types, of which only three are of interest in comparison with our experimental date ${ }^{3}$

The VDEs of the surface isomer are in good agreement with isomer Ia, except for the largest cluster with $n=200$. With the single fit analysis the agreement holds only up to size 40 .

The partially embedded isomer represents a new solvation scheme for the excess

however, strongly from all other data and even from each other (LGS vs. TB), that we refrain from including them here.

${ }^{2}$ The data of the early Landman paper[5] is not included.

${ }^{3}$ The VDEs of the dipole-bound isomer are mostly lower than $0.5 \mathrm{eV}$ and correspond therefore best to isomer III found by the Neumark group for very cold $\left(\mathrm{D}_{2} \mathrm{O}\right)_{\mathrm{n}}{ }^{-}[77]$. 

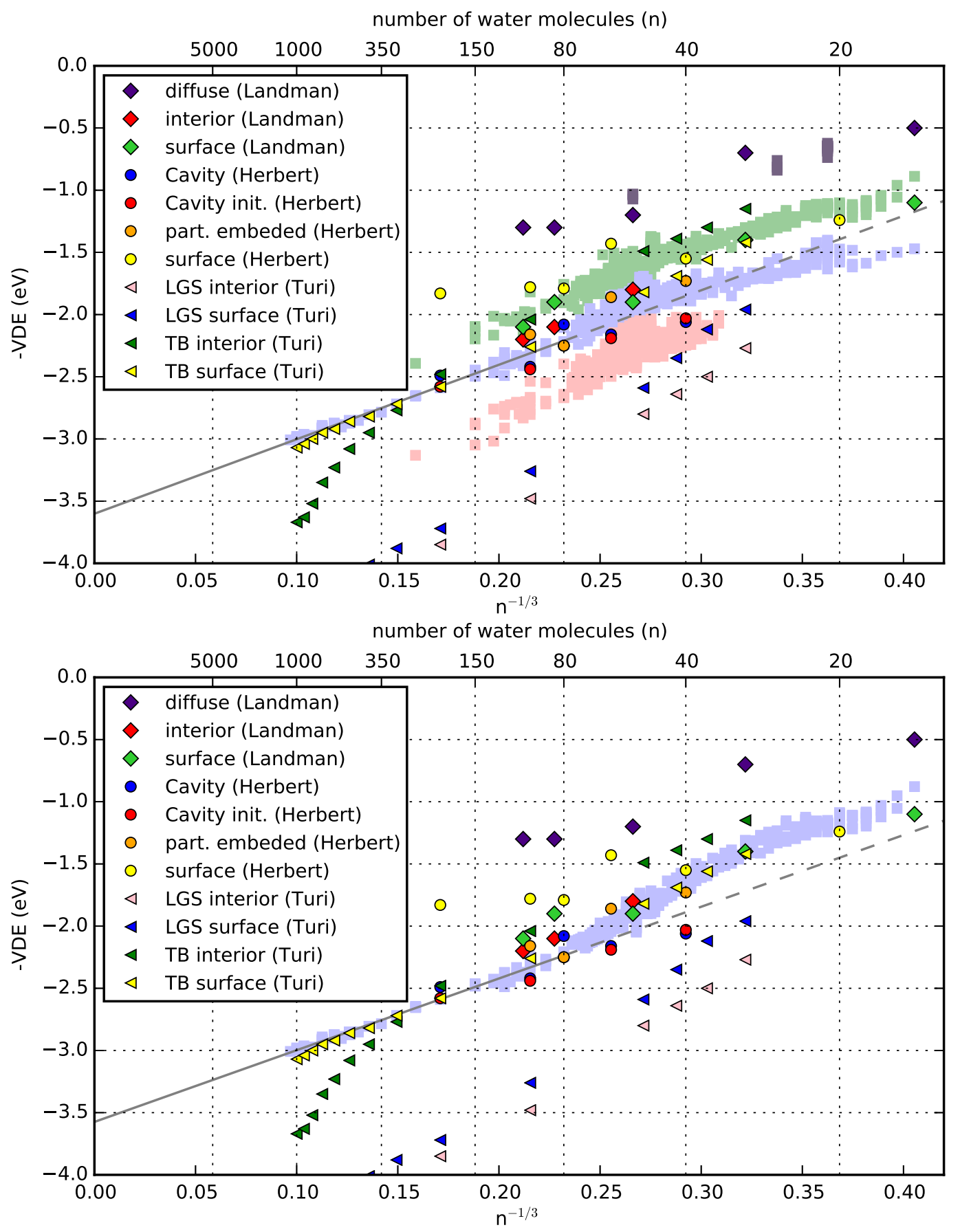

Figure 4.32.: Comparison of calculated VDEs of $\left(\mathrm{H}_{2} \mathrm{O}\right)_{\mathrm{n}}{ }^{-}$with the results of the multi GL analysis (upper panel) and the single GL analysis (lower panel). 
electron, in which the electron is localized in a partially formed solvent cavity at the cluster surface. The calculated VDEs show some overlap with the experimental VDEs of isomer Ib for 40, 60, and 80, while the value for size 100 is slightly too low. However, most values are lying more on the fringes of the experimental bands.

The VDEs of the interior or cavity localized electron are in good agreement with isomer Ib starting from size 60. The VDEs labeled "cavity init." represent calculations, which were initialized from a cavity state extracted from a previous simulation of the bulk hydrated electron. The "cavity" results were initialized from a neutral clusters, also based on bulk structure, which were equilibrated prior to electron attachment. It should be noted that the "cavity init." VDE of size 80 coincides in the plot with the partially embedded one and is therefore hardly visible. The same applies for the VDEs of size 200, which have almost the same values as "TB interior (Turi)" and "TB surface (Turi)". In the cases, where the cavity variants diverge from each other, the "cavity init." results show a better agreement.

The LGS-based VDEs calculated by Turi show almost no overlap with any experimental data measured so far. A much better agreement, even though controversial, can be found for the TB-based VDEs. Conspicuous is the change in slope starting around size 400 exhibited by both models for the interior localized electron ${ }^{4}$, which explains the strong deviation of the slopes in table 4.1 .

The VDEs of the TB interior state for large sizes show therefore a good agreement with isomer Ib from 200 only up to 400. For smaller sizes between 35 and 100 they fit better with isomer Ia. This is due to the fact that in this size range the TB interior VDEs are lower than the ones of the TB surface state.

The TB surface state VDEs agree astonishingly well with the VDEs of the single fit analysis (or isomer I). This means that in comparison to the multi GL analysis, the VDEs of the small sizes correspond to isomer Ia, lie between Ia and Ib around 40 , and, starting with 50 , coincide almost perfectly with isomer Ib even up to very large sizes.

\subsubsection{Dielectric Sphere Model and Permittivities}

In the dielectric sphere model (cf. chap. 3) the slope $A$, which describes the evolution of the VDEs with the cluster size, is a function of the frequency-dependent relative permittivity $\epsilon_{r, \nu}:=\epsilon_{r}(\nu)$, the relative static permittivity $\epsilon_{r, s}:=\epsilon(\nu=0)$ and the mean radius of the water molecule $r_{b u}$ (eq. (3.3)). Having determined the slope $A$ experimentally, allows one to calculate one permittivity from the other one,

${ }^{4}$ The VDEs of "LGS interior"-state are not visible in our plot, but run basically parallel to the "TB interior" VDEs (cf.[73]). 
deploying e.g. published values for the bulk:

$$
\begin{aligned}
\epsilon_{r, \nu} & =\left(\frac{A}{k}-1+\frac{2}{\epsilon_{r, s}}\right)^{-1}, \\
\epsilon_{r, s} & =2\left(1-\frac{A}{k}+\frac{1}{\epsilon_{r, \nu}}\right)^{-1},
\end{aligned}
$$

where $k:=e^{2} /\left(2 r_{b u}\right)$. Barnett et al. utilized in their publication $k=4.43$ and the permittivities for liquid water at $300 \mathrm{~K}, \epsilon_{r, s}=78.5$ and $\epsilon_{r, \nu}=1.78$.

Water has a highly temperature dependent relative static permittivity $\epsilon_{r, s}$ ranging from 55.5 at $100^{\circ} \mathrm{C}$ to 88.7 at $0{ }^{\circ} \mathrm{C}[22]$. When dealing with water clusters, the permittivities of water ice might be the better estimate. The relative static permittivity of ice depends of the type of ice. The "common" ice at atmospheric pressures is the crystalline type $I_{h}$ with hexagonal structure, which is stable down to temperatures of about $80 \mathrm{~K}$ to $130 \mathrm{~K}[19$. Its relative static permittivity ranges from 90 at $273 \mathrm{~K}$ and 180 at $150 \mathrm{~K}[37$. At lower temperatures (and low pressure) low density amorphous ice (LDA) is the most common structure of ice, e.g. in the interstellar medium[19]. Data about its relative static permittivity is scarce, but it seems to have a much lower value: Andersson found it to be below 10 at $0.1 \mathrm{~Hz}$ and 50 bar[1].

For non-ferromagnetic media (with relative permeability $\mu_{r} \approx 1$ ) the frequencydependent permittivity can be derived from its refractive index $n(\nu)$ by $\epsilon_{r, \nu}=$ $n(\nu)^{2}[20]$. The values of the refractive indexes of water, $I_{h}$ and LDA ice for visible and near UV light respectively lie all in the same range between 1.27 (LDA) to 1.35 $\left(I_{h}\right)[12,78]$, which maps to a $\epsilon_{r, \nu}$ range of 1.61 to 1.82 .

On account of the highly varying values of the permittivities and the uncertainties about the cluster structure and the mean radius of the $\mathrm{H}_{2} \mathrm{O}$ molecule in the cluster, it is not possible to derive actual numbers from the dielectric sphere model. It is, however, instructive to look at a certain value range and the impact different slopes $A$ have on the results.

Since the range of plausible values for the frequency-dependent permittivity is much smaller, we can start with eq. 4.5 and plot $\epsilon_{r, s}$ as a function of $\epsilon_{r, \nu}$ for different slopes $A$ (fig. 4.33). The authors of the original publication [5] used $k=$ 4.43 , which corresponds to a unusual high density of $1.6 \mathrm{~g} / \mathrm{cm}^{3}$. We choose $k=3.8$ instead, according to the common density of $1 \mathrm{~g} / \mathrm{cm}^{3}$. The slope values $A$ represent room temperature water ${ }^{5}(A=5.84)$ and the results of the least square fits to our experimental data from the single and multi GL fit analysis respectively (5.75 and 6.0). Additionally a slope of $A=3$ is plotted, which would be required to connect the VDE of our largest water cluster $\left(\left(\mathrm{H}_{2} \mathrm{O}\right)_{1100}{ }^{-}\right)$to a bulk value of $3.3 \mathrm{eV}$, which marks the lower boundary of the range of experimental water-jet results.

\footnotetext{
${ }^{5}$ Using $\epsilon_{r, s}=78.5$ and $\epsilon_{r, \nu}=1.78$.
} 


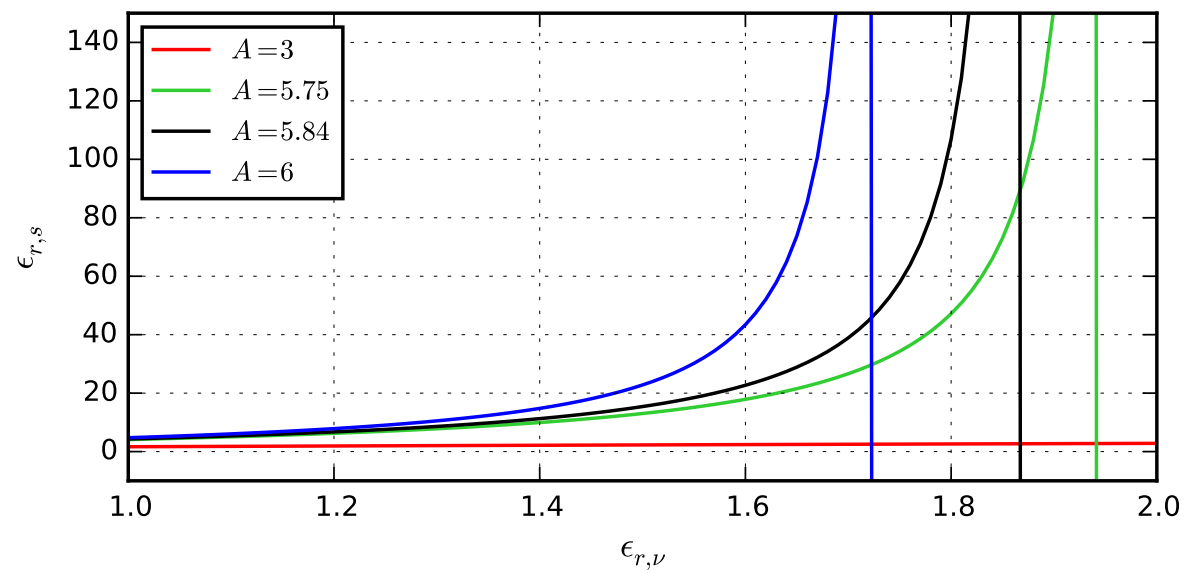

Figure 4.33.: The relative static permittivity as function of the frequency-dependent relative permittivity for various slope values $A$.

The slope values from the experimental data result in similar, but slightly shifted $\epsilon_{r, s}$ progressions to the liquid water case. They exhibit a steep rise for an input range of 1.6 to $1.7(A=6)$ and 1.8 to $1.9(A=5.75)$ respectively, yielding $\epsilon_{r, s}$ values between 40 and 120 . By contrast stays $\epsilon_{r, s}$ always below 5 for $A=3$.

In fig. 4.34 the frequency-dependent relative permittivity is plotted as a function of the relative static permittivity (eq. 4.4)). The inset shows an enlargement for the

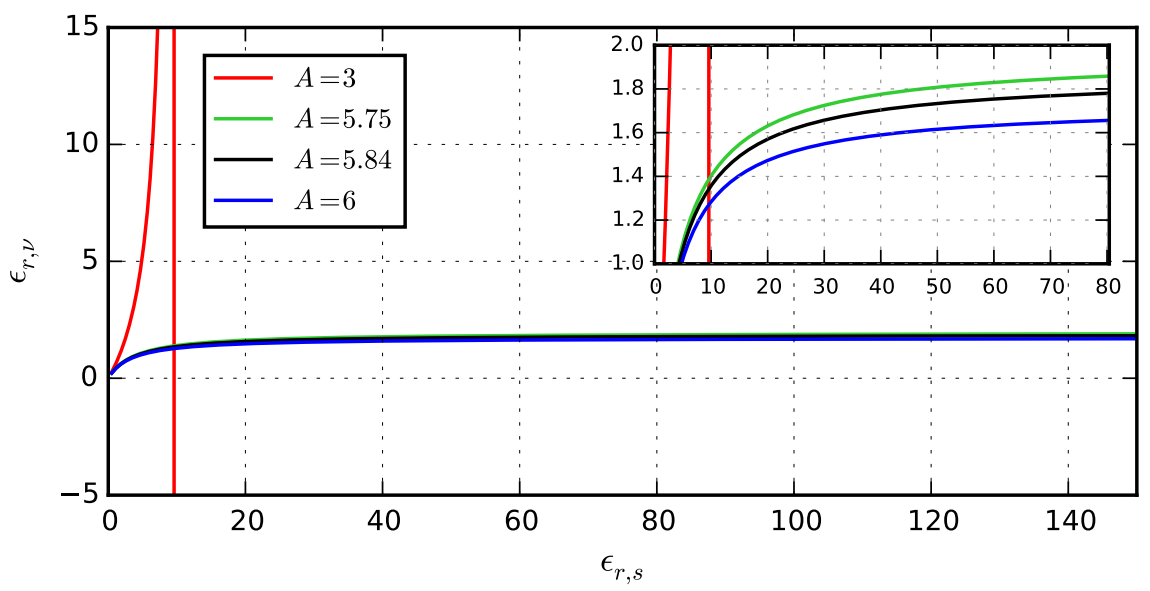

Figure 4.34.: The frequency-dependent permittivity as function of the relative static permittivity for various slope values $A$.

area with $\epsilon_{r, s} \leq 80$. The three slope values between 5.75 and 6 result in an $\epsilon_{r, \nu}<2$, whereas larger changes occur only for $\epsilon_{r, s}$ ranging below 10 . The hypothetical slope value $A=3$ shows again a distinctly different progression. Meaningful values 
result only from a very small input range, which is limited due to a discontinuity at $\epsilon_{r, s} \approx 10$ and negative $\epsilon_{r, \nu}$ for larger $\epsilon_{r, s}$. 



\section{Heavy Water Cluster}

Heavy water or deuterated water $\left(\mathrm{D}_{2} \mathrm{O}\right)$ consists of water molecules, where the hydrogen isotope deuterium replaces the hydrogen atoms. It has generally very similar chemical and physical properties to normal water, but exhibits also some differences due to the higher mass of the deuterium. The nucleus of deuterium contains one proton and one neutron and is therefore almost twice as heavy as the nucleus of normal water. Thus the molecular vibrations are at lower frequencies as in light water and the zero point energy is lower too. As a further consequence subtle differences in the inner and intermolecular bond lengths of oxygen and hydrogen and deuterium respectively has been found in bulk water[69. So is the $\mathrm{OH}$ bond length a few percent larger than the OD bond, which shortens the hydrogen bond in normal water in contrast to heavy water.

Heavy water cluster anions $\left(\mathrm{D}_{2} \mathrm{O}\right)_{\mathrm{n}}{ }^{-}$represents therefore an interesting complementary measurements to non-deuterated water clusters. Due to the similarities of heavy and normal water, the measurements should yield very similar results. However, the increased weight of deuterium may unfold small differences, especially concerning vibration related aspects of the spectrum.

\subsection{Experimental Data}

Heavy water cluster anions $\left(\mathrm{D}_{2} \mathrm{O}\right)_{\mathrm{n}}{ }^{-}$were produced analogously to $\left(\mathrm{H}_{2} \mathrm{O}\right)_{\mathrm{n}}{ }^{-}$by replacing water with deuterated water $\mathrm{D}_{2} \mathrm{O}$ in the cluster source. Photoelectron spectra of mass selected cluster anions were recorded for all sizes from $n=18$ to $n=55$ and for $n=60$. For all spectra an excitation laser wave length of $308.4 \mathrm{~nm}$ $(4.02 \mathrm{eV})$ was used. The clusters were usually thermalized at a trap temperature of $10 \mathrm{~K}$. Exceptions are the sizes 21 and 48, for which temperature dependent measurements were conducted additionally.

Over the measured size range the recorded spectra show in general a great resemblance with their $\left(\mathrm{H}_{2} \mathrm{O}\right)_{\mathrm{n}}{ }^{-}$counterparts, that is one single broad and asymmetric peak (fig. 5.1). As for the water clusters, some sizes deviate from that general line shape and exhibit small pre-peaks or additional shoulders. Small pre-peaks are most notably visible for the sizes 21, 26, and 53 but also show up for 24 and 25 . Shoulders are found in the size range from about 45 to 53 , which also coincides with the findings of normal water clusters. A full listing of $\left(\mathrm{D}_{2} \mathrm{O}\right)_{\mathrm{n}}{ }^{-}$photoelectron spectra can be found in sec. A.4 in the appendix. 

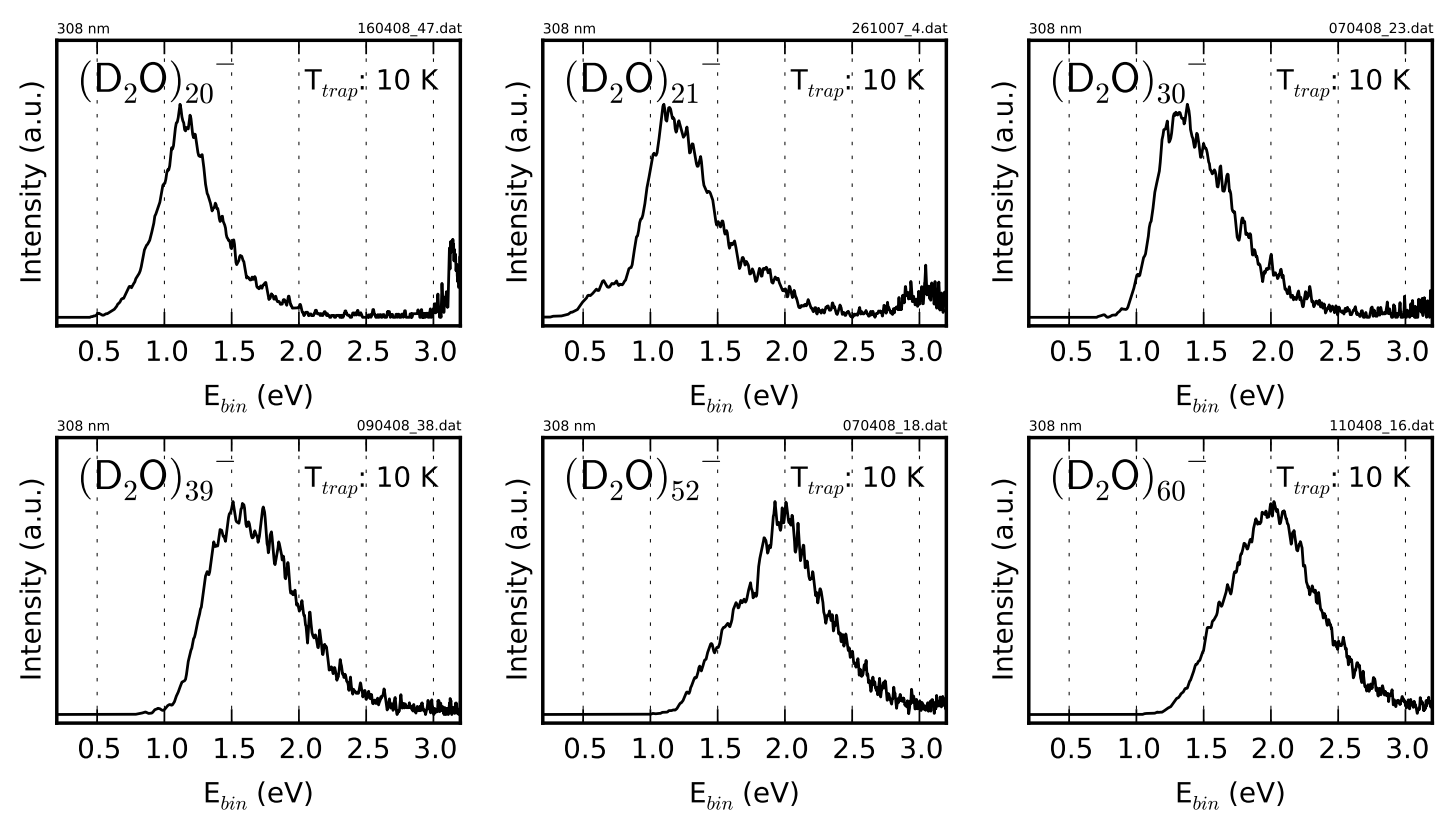

Figure 5.1.: Example photoelectron spectra of heavy water cluster anions $\left(\mathrm{D}_{2} \mathrm{O}\right)_{\mathrm{n}}{ }^{-}$.

\subsection{Peak Analysis}

The peak analysis of the $\left(\mathrm{D}_{2} \mathrm{O}\right)_{\mathrm{n}}{ }^{-}$photoelectron spectra was conducted in the same way as for the water cluster anions (cf. sec. 4.2.1). Given the apparent similarities of the spectra, we can expect the results of the analysis to be likewise similar. We chose, however, to proceed along the lines of the water chapter, to be able to compare the results of the different steps of the analysis directly. To ease the comparison, all VDE plots in this chapter include also the results of the corresponding analysis of normal water clusters, plotted faded in the background.

\subsubsection{Single GL Fit Analysis}

The single fit analysis of the heavy water cluster spectra reveals the same shortcomings as for the water clusters. The spectral line shape of most sizes is only approximately reproduced by a single Gaussian-Lorentzian function.

The results of the peak analysis is pooled in fig. 5.2. The left panel shows the vertical detachment energy (VDE), the right panel the peak width ( $f w h m)$ and the width parameter $\sigma_{G}$ and $\sigma_{L}$ in dependency of the inverse cluster radius $n^{-1 / 3}$. The VDEs increase steadily with the cluster size, but not in a clear linear progression. Exceptional is again the size range 50 to 55, were the VDE shifts considerably from size to size.

The evolution of the line shape parameters follows also the trend found for the water clusters. The fwhm raises with the size without large shifts. Exceptions are 

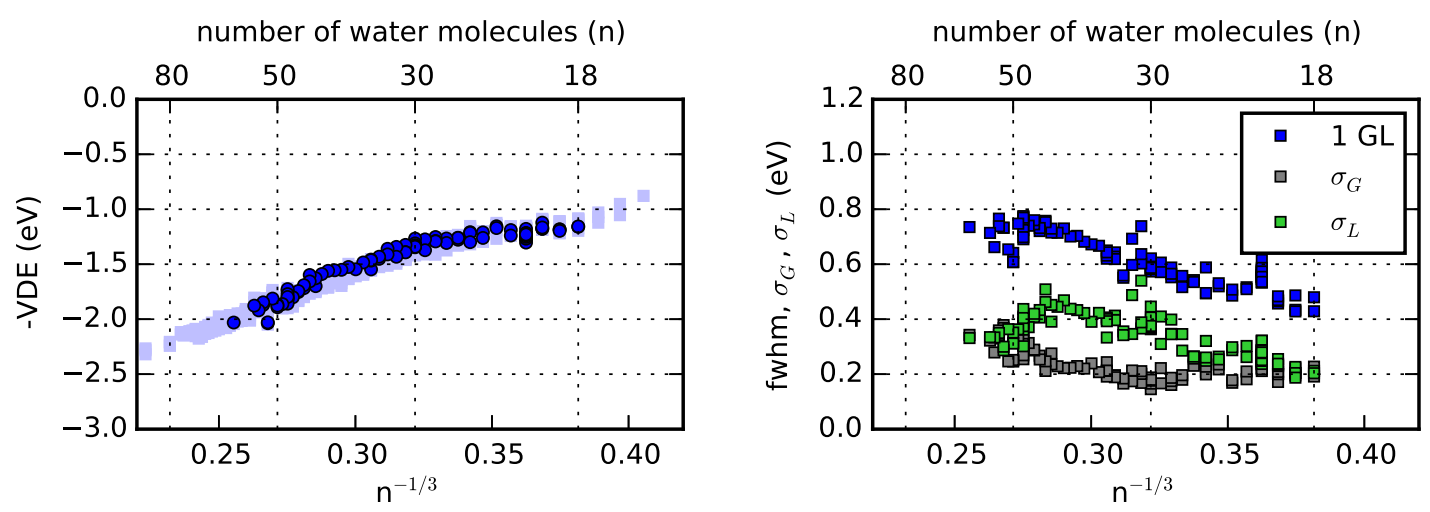

Figure 5.2.: Evolution of the VDEs (left) and width parameters (right) of $\left(\mathrm{D}_{2} \mathrm{O}\right)_{\mathrm{n}}{ }^{-}$ with the inverse cluster radius (single GL fit analysis).

the size 21, which is wider due to its pronounced pre-peak, and the sizes 33, 50, and 51, which are narrower compared to their neighbors.

\section{Impact of Deuteration on the Single Fit Results}

The previous section has shown that the spectra and the evolution of the spectral features are qualitatively largely similar. But are there also quantitative similarities? Let's start by comparing the VDEs ascertained from the single GL fit analysis (fig. 5.2, left). For almost all sizes one finds that the VDEs are identical within the accuracy of measurement and analysis. Even the highly fluctuating VDEs of the sizes 50 to 55, match exactly. Only the VDEs between 39 to 46 are lower by $50 \mathrm{meV}$ to $150 \mathrm{meV}$.

The line shape parameter show a different picture (cf. fig. 4.2.3, lower panel and 5.2 right): the peak width of the deuterated cluster spectra is smaller for all cluster sizes by about $50 \mathrm{meV}$ to $120 \mathrm{meV}$. Only exceptions are the two smallest clusters in our measurements, 18 and 19, which are actually broader. The minor width is a result of the smaller width of the peak on the Lorentzian side $\sigma_{L}$ for almost all sizes, whereas the Gaussian width parameters $\sigma_{G}$ do not change upon deuteration. An exception from this rule form the spectra of the sizes 39 to 46 , for which $\sigma_{G}$ of the deuterated clusters is also considerable smaller, than for the "normal" water clusters. The opposite happens to the small cluster sizes, 18 and 19, which exhibit a larger $\sigma_{G}$ upon deuteration, which in turn is the main contribution to the greater width.

Although the positions of the peak maximum of the deuterated water clusters are in very good agreement with their "normal" counterparts, the line shapes deviate considerably. Due to the smaller $\sigma_{L}$ they assume generally a more symmetric shape. However, for some sizes, like 18 to 21,24 to 26 , and > 49, this leads to an "inverse" asymmetry, where the width of the Gaussian part is larger than the width of the 
Lorentzian.

As in the case of the water clusters, the single fit analysis of the deuterated cluster spectra is not capable to adapt to the individual details of the spectral shapes. This leads to high fluctuations of the general peak shape, even to the point where the asymmetry is reversed. Consequently show the heavy water clusters even stronger indications that more than one GL function is needed to adequately fit the spectra.

\subsubsection{Multi GL Fit Analysis}

\section{GL Fit Analysis}

Fitting the main peak of the deuterated water cluster spectra with 2 GL functions distinctly enhances the agreement of the fit with the experimental data. Analogous to the water spectra, the main peak is formed for the small $\left(\mathrm{D}_{2} \mathrm{O}\right)_{\mathrm{n}}{ }^{-}$by a dominant peak (Ia) and a additional, small peak at higher binding energies (Ib). The Ib peak starts growing at approximately $n=34$. It reaches the same intensity at sizes 47 or 48 and is the dominant peak for all sizes $>50$. The sizes 50, 51, and 54 do not have the low binding energy peak Ia anymore, but rather show a high binding energy peak (HE) in addition to the dominant Ib peak. It should be noted that this is not as much caused by a particularly prominent shoulder on the high binding energy slope, but rather how the 2 GL fit adapt to the clearly missing low binding energy shoulder.

The sizes 21, 26, and 53 feature distinct pre-peaks (peak II), analogously to the non-deuterated water clusters. Additionally show the spectra of $\left(\mathrm{D}_{2} \mathrm{O}\right)_{24}{ }^{-}$and $\left(\mathrm{D}_{2} \mathrm{O}\right)_{25}{ }^{-}$also such pre-peaks, in contrast to the normal water spectra.

The resulting energies of the peak maxima form two well separated bands (fig. 5.3 . upper panel). Both bands show a linear trend with the cluster radius between 22 and 45. The size range 50 to 55 shows again large fluctuations of the VDEs in comparison to their neighbors.

The lower panel of fig. 5.3 displays the line shape parameters. Both width parameters, $\sigma_{G}$ and $\sigma_{L}$, evolve with fewer large shifts, than in the single GL case. $\sigma_{G}$ keeps in general its value and evolves from $100 \mathrm{meV}$ to $250 \mathrm{meV}$. Especially the progression around 21 and 24 to 26 is more continuous, due to factoring in the pre-peaks (II) with an additional GL functions.

The Lorentzian width $\sigma_{L}$ is for most sizes much smaller than in the single fit approach, ranging from $100 \mathrm{meV}$ to $330 \mathrm{meV}$. Accordingly is the full width also reduced to $400 \mathrm{meV}$ to $550 \mathrm{meV}$, in contrast to $400 \mathrm{meV}$ to $800 \mathrm{meV}$ of the single fit analysis.

Since $\sigma_{L}$ becomes smaller when using 2 fit functions for the main peak and $\sigma_{G}$ retains its value, the issue of reversed peak shape asymmetries becomes more pronounced for some cluster sizes, in particular the small sizes 18 to 21, as well as 27,35 , and some in the 50 to 55 range. 

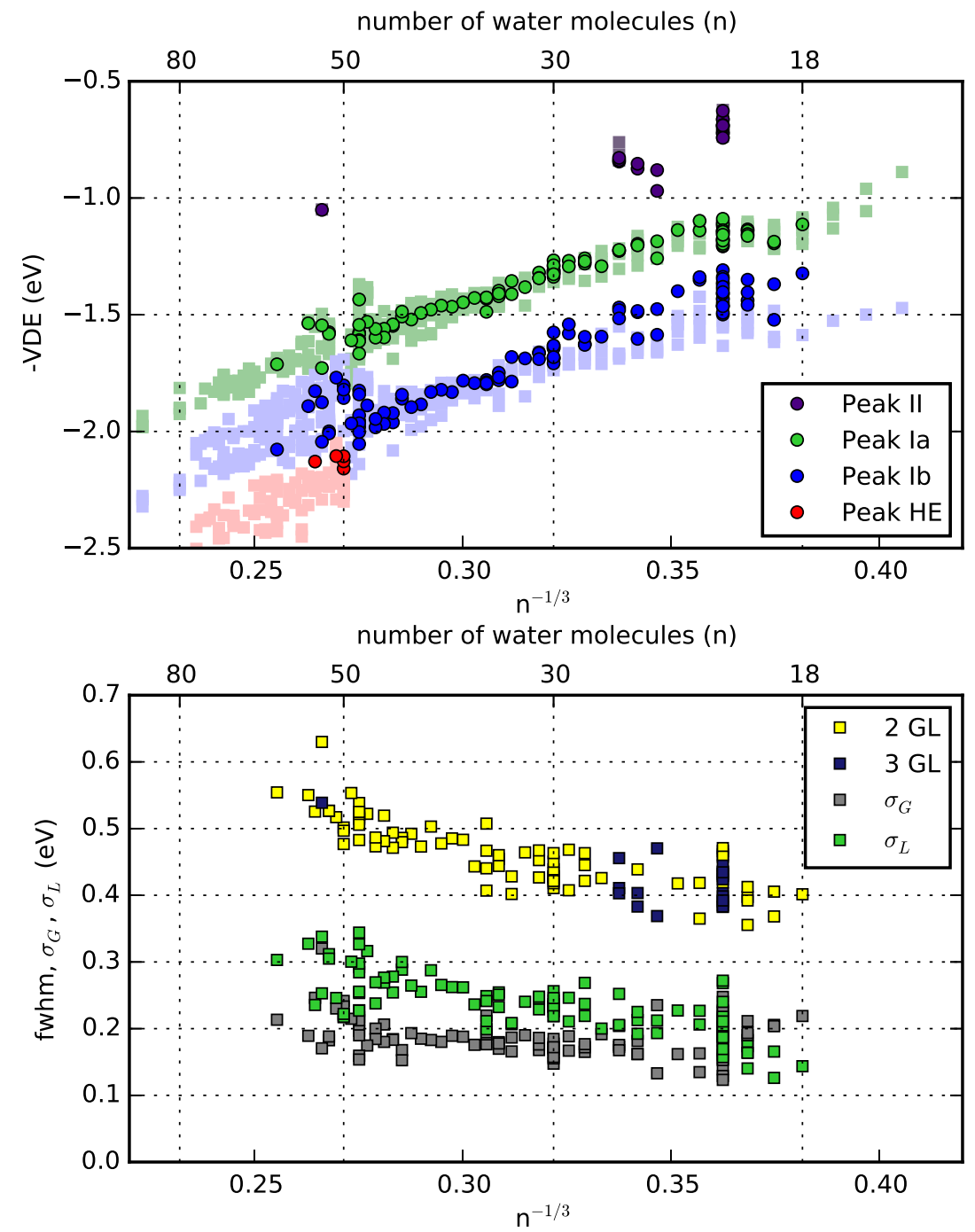

Figure 5.3.: Development of the VDEs (upper panel) and width parameters (lower panel) with the cluster size (2 GL fit analysis). 


\section{Comparison with the $\left(\mathrm{H}_{2} \mathrm{O}\right)_{\mathrm{n}}{ }^{-} 2 \mathrm{GL}$ Fit Results}

The overall picture of the peak evolution gained by the 2 GL fit analysis of the $\left(\mathrm{D}_{2} \mathrm{O}\right)_{\mathrm{n}}{ }^{-}$spectra is very similar to one of water clusters: For small sizes the main contribution is from the peak Ia, with an additional, much smaller contribution on its high binding energy slope by peak Ib. This higher binding energy peak Ib grows in intensity with increasing cluster size. Both, the onset of the intensity growth and the size, were Ia and Ib reach the same intensity, occur, however, at slightly larger sizes for the deuterated water clusters. The low binding energy peak II could be identified for two more sizes in the case of the heavy water.

Comparing the VDEs shows an excellent agreement for the peaks II and Ia, as well as for peak Ib for sizes $>32$, and the additional high binding energy peak HE found for 50 and 51. This means that the deviations for the sizes 39 to 46 found in the single fit analysis do not exist anymore. The VDEs of peak $\mathrm{Ib}$ of $\left(\mathrm{D}_{2} \mathrm{O}\right)_{\mathrm{n}}{ }^{-}$, with $n<33$, are shifted to lower binding energies by about $50 \mathrm{meV}$ to $250 \mathrm{meV}$ in comparison to $\left(\mathrm{H}_{2} \mathrm{O}\right)_{\mathrm{n}}{ }^{-}$. In chapter 4.2.5 we used these differences of the peak distances already to discuss the origin of peak Ib. Cf. fig. 4.21, p. 66, which shows the development of the $\Delta E_{\mathrm{H}_{2} \mathrm{O}} / \Delta E_{D_{2} \mathrm{O}}$ ratio with the cluster size.

The comparison the peak shape parameters shows that the main difference is the smaller width $\sigma_{L}$ of the Lorentzian side of the peak. Starting at size 22 the value of $\sigma_{L}$ is about $30 \mathrm{meV}$ to $100 \mathrm{meV}$ lower for almost all sizes. The width parameter of the Gaussian part $\sigma_{G}$ is in this size range in very good agreement with the nondeuterated one. This leads to a reduced $f w h m$ of the heavy water peaks of the same order of magnitude as $\sigma_{L}$. The peak shape parameter of the small cluster sizes 18 to 21 deviate considerably, because the deuterated clusters tend to have an inversed asymmetry. This means $\sigma_{G}$ is larger and $\sigma_{L}$ is smaller than for the non-deuterated case.

\section{Multi GL Analysis}

As in the case of the non-deuterated water, there are spectra, whose main peak might be fitted even better with 3 GL functions. The Lorentzian slope of the sizes $>51$ show a s-like deviation, where the fit first passes under and later over the experimental data. Also vanishes for many sizes between 37 and 49 the high binding energy slope faster than the fit. These are only vague hints and they are less clear as in the case of normal water. But since we have undertaken such an analysis for the non-deuterated water clusters, it might be interesting to investigate the impact of deuteration.

For reasons of comparability and analogy with approach 2 of the multi GL analysis of $\left(\mathrm{H}_{2} \mathrm{O}\right)_{\mathrm{n}}{ }^{-}$, we have fitted the main peak with 3 GL for sizes with $n>27$, except sizes 50 and 51. Additionally the sizes 18 to 20 were fitted with 3 GL functions, in order to verify, if this could explain the reversed asymmetry found for the 

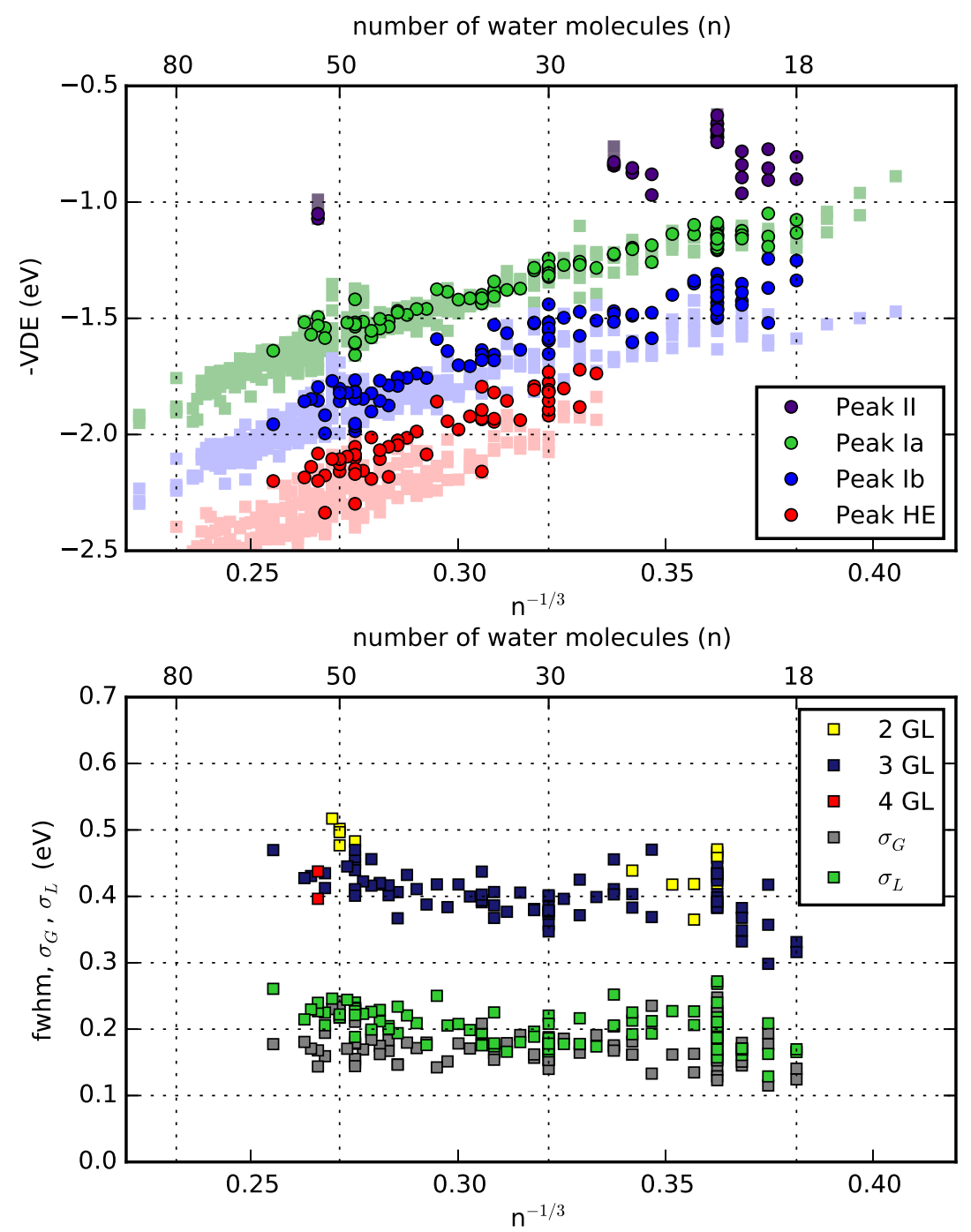

Figure 5.4.: Size dependent evolution of the VDEs (upper plot) and width parameters (lower plot) resulting from a multi GL analysis.

2 GL fits. The qualitative improvement of the fits is rather low. Only for the sizes from 52 and higher adapts the fit better to the experimental data, especially in the high binding energy region. Fig. 5.4 summarizes the fit results in the usual plots of the VDEs (upper panel) and peak shape parameter (lower panel) versus the inverse cluster radius. For the small clusters (18 to 20) appear additional low binding energy peaks (peak II?), which are located at considerably higher binding energies, than the corresponding peak of $\left(\mathrm{D}_{2} \mathrm{O}\right)_{21}{ }^{-}$. The VDEs of peak Ia and Ib are not affected by the these peaks and remain in good approximation the same as for the 2 GL fit. For the larger clusters starting at 27, the additional peak appears at the high binding energy slope. While the VDEs of peak Ia shifts only very slightly by 
about $10 \mathrm{meV}$ to $80 \mathrm{meV}$ to lower binding energies, peak Ib is much more effected and shifts about $50 \mathrm{meV}$ to $150 \mathrm{meV}$. Only for the sizes starting at 52 is the impact on the VDEs of peak Ia and Ib small, when switching from 2 to 3 GL functions.

The peak widths are again reduced, when using a 3 GL fit (fig. 5.4. lower panel). The width stays almost constant around $0.4 \mathrm{eV}$ for the size range from 21 to 46 . There is a minimal increase for the sizes $>51$, whereas the size 50 and 51 are much wider, because they were fitted by only 2 GL functions. For the small clusters, 18 to 20 , the decrease of the width can be attributed mainly to the considerable smaller $\sigma_{G}$. However, $\sigma_{L}$ is of the same order of magnitude, so that the asymmetry is still reversed. For the sizes $>26$ the decrease of the Lorentzian width is the main cause for narrower $f w h m$ value. It thus follows the scheme, we have found for the 2 GL fits. The 3 GL fits also reduce the value of the Gaussian width, even though only to a small degree (up to $30 \mathrm{meV}$ ). The stronger decrease of $\sigma_{L}$ causes it to adapt values similar to $\sigma_{G}$, that means that now almost all sizes between 27 and 49 assume a line shape with a reversed asymmetry. For the sizes $>51$ this effect is reversed and they show less tendency towards a reversed asymmetry.

\section{Comparison to the $\left(\mathrm{H}_{2} \mathrm{O}\right)_{n}{ }^{-}$Multi GL Fit Results}

The VDEs of peak II and peak Ia are in excellent agreement for all measured cluster sizes with the VDEs of normal water. The peak Ib VDEs of the deuterated clusters are distinctly lower for for the small sizes up to 40. They approach the normal water values between 40 and 50, and are identical within the accuracy of this analysis for sizes starting at 50. The peak HE VDEs are always smaller for all sizes. These relations are expressed in fig. 5.5 by plotting the ratios of the energy differences $\Delta E_{\mathrm{H}_{2} \mathrm{O}} / \Delta E_{D_{2} \mathrm{O}}$ for peaks Ia and Ib (left panel) and for peaks Ib and $\mathrm{HE}$ (right panel).

A comparison of the line shape parameter reveals that the deuterated peak width is narrower by about $100 \mathrm{meV}$. This conforms with width reduction found in the 2 GL analysis. Another parallelism is that these differences are mainly caused by the lower value of $\sigma_{L}$ of the deuterated clusters, while the Gaussian width parameters $\sigma_{G}$ are almost identical.

\subsubsection{Temperature Dependency}

The temperature dependency of $\left(\mathrm{D}_{2} \mathrm{O}\right)_{\mathrm{n}}{ }^{-}$has been investigated in few cases only, namely for sizes 21 and 48. In both cases the spectra show a similar evolution with the temperature as their non-deuterated counterparts. The plots summarizing the results of the analysis can be found in chapter $\mathrm{B}$ in the appendix.

The pre-peak (peak II) of $\left(\mathrm{D}_{2} \mathrm{O}\right)_{21}{ }^{-}$is also only visible in spectra recorded at temperatures up to $120 \mathrm{~K}$, whereas the VDEs of the main peak show no temperature 

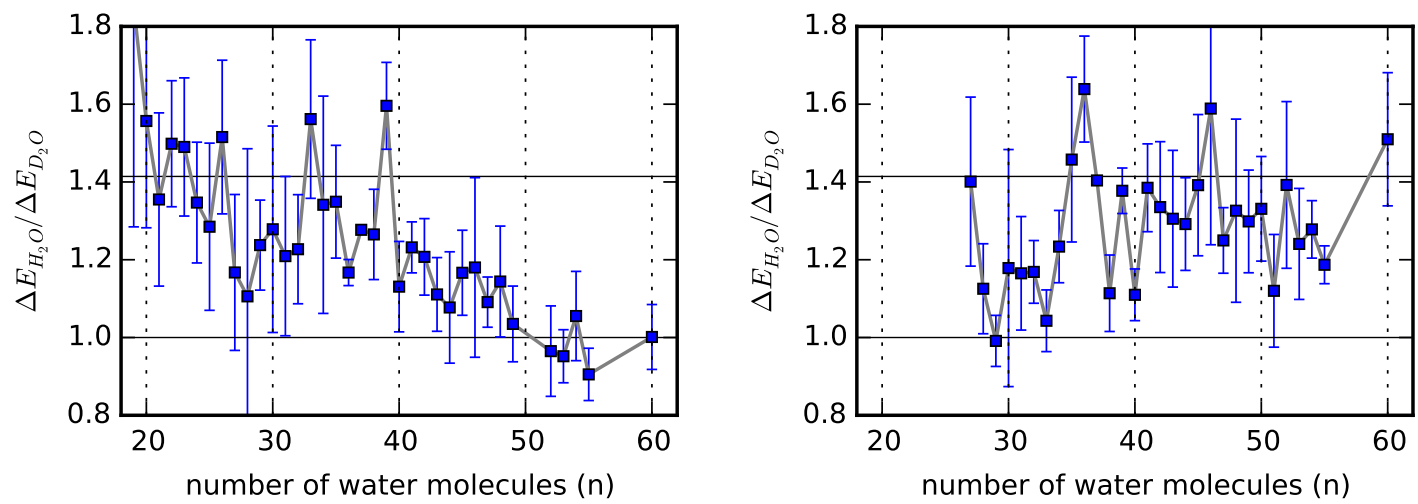

Figure 5.5.: Development of the energy offset ratios with the cluster size, when using results of the multi GL fit analysis. Left: ratios of the energy offset between peak Ia and Ib. Right: ratios of the peak offset between peak $\mathrm{Ib}$ and $\mathrm{HE}$.

dependency (cf. fig. B.17, p. 171). The same applies to the peak shape parameters, which remain constant over the whole temperature range.

The temperature dependent measurements of $\left(\mathrm{D}_{2} \mathrm{O}\right)_{48}{ }^{-}$are not as extensive as for $\left(\mathrm{H}_{2} \mathrm{O}\right)_{48}{ }^{-}$. The available data suggests, however, that the spectral evolution of the peaks with the temperature follows its non-deuterated counterpart (fig. 5.6). In particular shows the spectrum at $100 \mathrm{~K}$ the most structured shape of the main peak.

The temperature analysis of the VDEs (cf. fig. B.18, p. 172) underlines this observation by being almost identical with the analysis of $\left(\mathrm{H}_{2} \mathrm{O}\right)_{48}{ }^{-}$. Sole exception poses the measurement at $120 \mathrm{~K}$. The peak shape of that measurement deviates, however, so strong from all other measurements that an error may have occurred during recording and further measurements are necessary to verify this issue.

The analysis of the line shape parameters of $\left(\mathrm{D}_{2} \mathrm{O}\right)_{48}{ }^{-}$exhibits a similar temper-
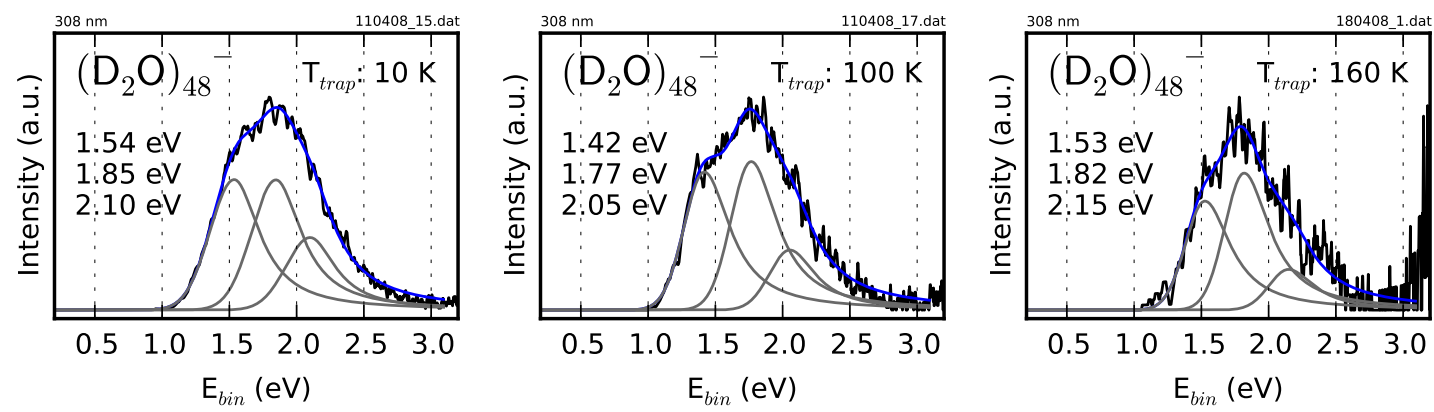

Figure 5.6.: Temperature dependent peak distribution of the photoelectron spectra of $\left(\mathrm{D}_{2} \mathrm{O}\right)_{48}{ }^{-}$using 3 GL fits. 
ature dependency as $\left(\mathrm{H}_{2} \mathrm{O}\right)_{48}{ }^{-}$. This means a decrease of $\sigma_{G}$ in the temperature range between $90 \mathrm{~K}$ and $130 \mathrm{~K}$. As for the VDEs, deviate the shape parameters for $n=120$ strongly from the general trend and the progression of $\sigma_{G}$ of the nondeuterated clusters and are therefore most likely invalid. $\sigma_{L}$ shows for both sizes no distinct temperature evolution, but it emphasizes again the differences in the line width between deuterated and non-deuterated clusters: Whereas $\sigma_{G}$ is of the same value, $\sigma_{L}$ is about $50 \mathrm{meV}$ to $100 \mathrm{meV}$ smaller for heavy water. This stays valid over the whole temperature range.

\subsection{Discussion}

Concerning the peak analysis of deuterated water clusters, we face the same dilemma, which we encountered for the non-deuterated clusters. The single fit approach fails to approximate the experimental spectra in a considerable size range, namely for the small sizes up to approximately 60 or 70 water molecules. The spectra in this size range show features, which suggests an underlaying substructure consisting of 2 and 3 peaks respectively. As a consequence of fitting those sizes with 2 or 3 GL functions, appear discontinuities in the size evolution of the peak shape parameters, whenever the number of fit functions changes. So none of the various fit approaches is able to deliver a fully consistent picture, how the experimental line shape arises from the underlaying peak distribution.

The peak analysis of the heavy water clusters exhibits another difficulty, which arises from the narrower peak width. Especially the $3 \mathrm{GL}$ analysis yields often results with reversed peak asymmetry, where the width of Gaussian part is larger than the width of the Lorentzian one. Following our considerations about the general shape of the photoelectron spectra of water clusters in chapter 3, such results must therefore be discarded as not plausible. As a compromise we choose to fit the main spectral peak with 2 GL functions for small water clusters and with 3 GL functions starting at size 44 (fig. 5.7). This way we gain a better approximation of the spectra for these larger sizes, while avoiding reversed asymmetries. On the backside, this leads to the already discussed discontinuities of $\sigma_{G}$ and especially $\sigma_{L}$. The evolution of the energy offset ratios (fig. 5.8) also suggests that the nature of the substructure is not entirely reflected by our analysis.

The peak shape analysis of the very small clusters sizes, 18 to 21, results always in reversed peak asymmetries, independently of the number of fit function used for fitting the main peak. The same applies to the 2 GL fit of the sizes 50 and 51.

The peak analysis of the deuterated clusters revealed many similarities to the non-deuterated clusters, but showed also few significant differences. The general peak distribution and evolution with the cluster size is basically the same. For the small sizes dominates peak Ia, which we assign analogously to normal water clusters to an isomer class Ia. From size 50 onwards peak Ib is dominant and represents 

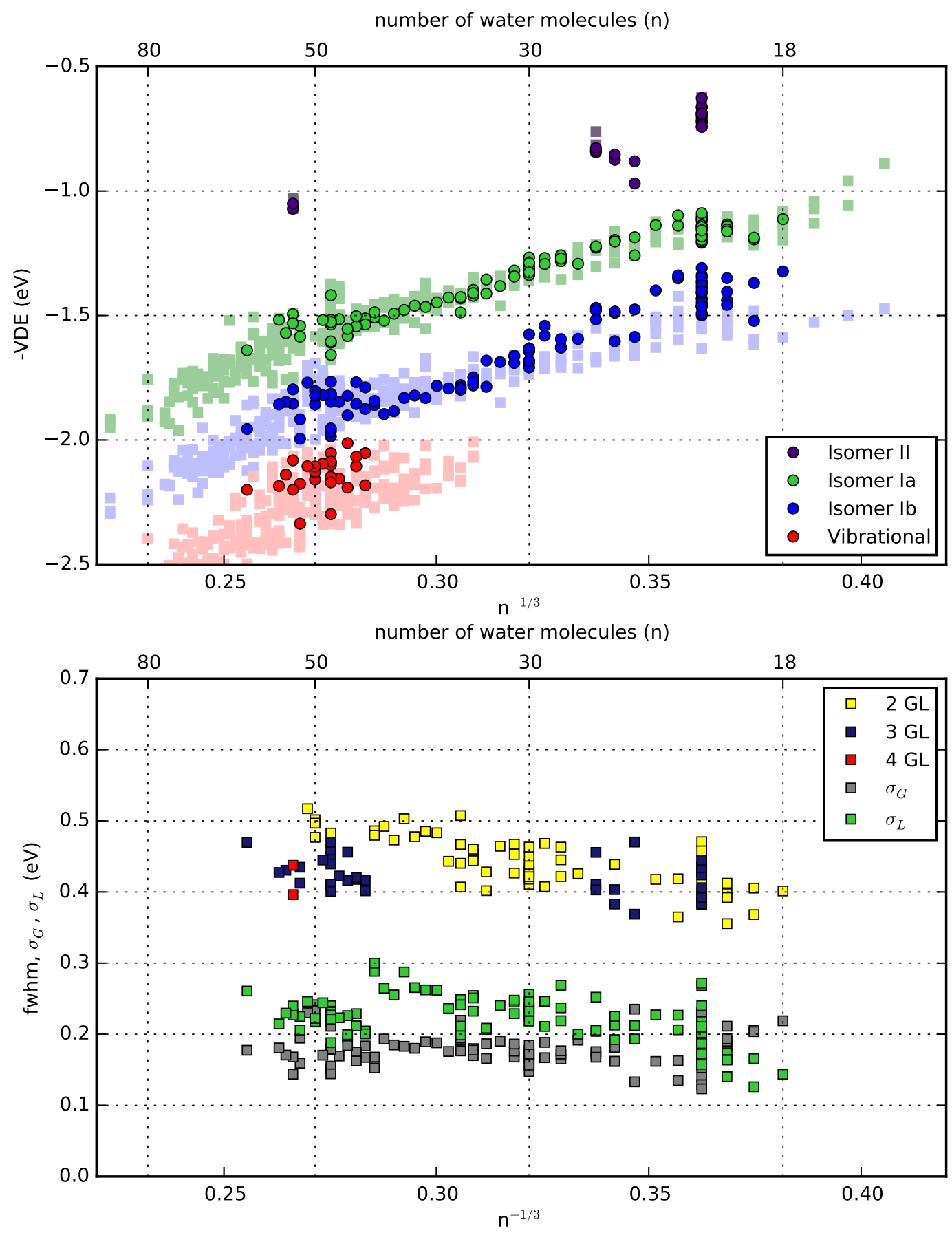

Figure 5.7.: Development of the VDEs (upper panel) and peak width parameters (lower panel) according to the multi GL analysis when using the "best compromise" number of GL functions. 

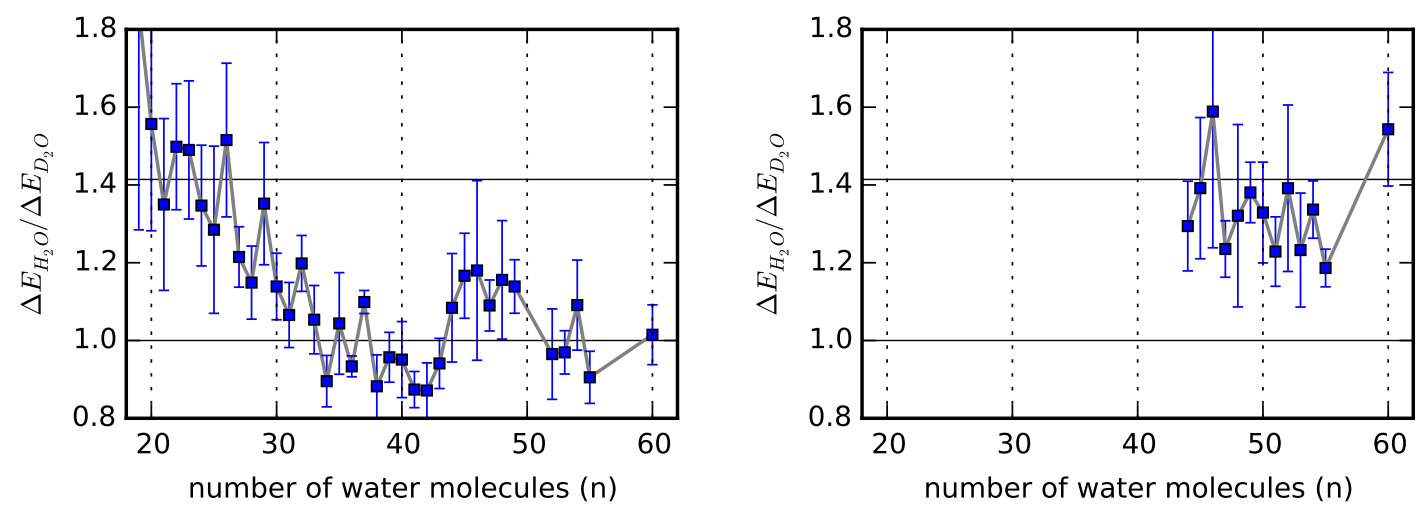

Figure 5.8.: Development of the energy offset ratios with the cluster size, when using results of the multi GL fit analysis with the "best compromise" number of GL functions. Left: ratios of the energy offset between peak Ia and Ib. Right: ratios of the peak offset between peak Ib and HE.

another isomer class Ib.

Difference between deuterated and non-deuterated water clusters are mainly found in the line shape of the spectra. Whereas $\sigma_{G}$ amounts for most sizes to a similar value, $\sigma_{L}$ is significantly smaller. Since the VDEs are also virtually identical one can assume that heavy and normal water clusters form the same structures. This leads to the conclusion that the Lorentzian tail of the spectra has its origin in the vibrational excitation of the clusters. The similarities of the temperature dependency of $\sigma_{G}$ found for $\left(\mathrm{H}_{2} \mathrm{O}\right)_{48}{ }^{-}$and $\left(\mathrm{D}_{2} \mathrm{O}\right)_{48}{ }^{-}$respectively indicates further that the Gaussian part of the main peak is defined primarily by the distribution of the isomer class.

\section{Comparison with experimental data of other groups}

External data of $\left(\mathrm{D}_{2} \mathrm{O}\right)_{\mathrm{n}}{ }^{-}$is primarily available for isomer II and III respectively [77], which seem to form more easily under cold experimental conditions. The isomer I data is for our spectra more interesting, but unfortunately less extensive. The upper panel of fig. 5.9 shows published isomer I[17] and isomer II VDEs[77] in comparison with the results of our multi GL peak analysis. The VDEs of isomer I from Coe et al. are in excellent agreement with the VDEs found for isomer Ia in our spectra. The same applies to the vibrational peak, which coincides with our peak Ib. As in the case of light water, we consider peak Ib of vibrational origin for sizes smaller than 35 .

The isomer II VDEs are in good agreement for the sizes 21 and 53, but deviates for sizes 24 to 26 . In this size range the abundance of isomer II is very low in our measurements. A possible explanation could be that due to the different temperature conditions of our experiment, a different type of those metastable isomers is 

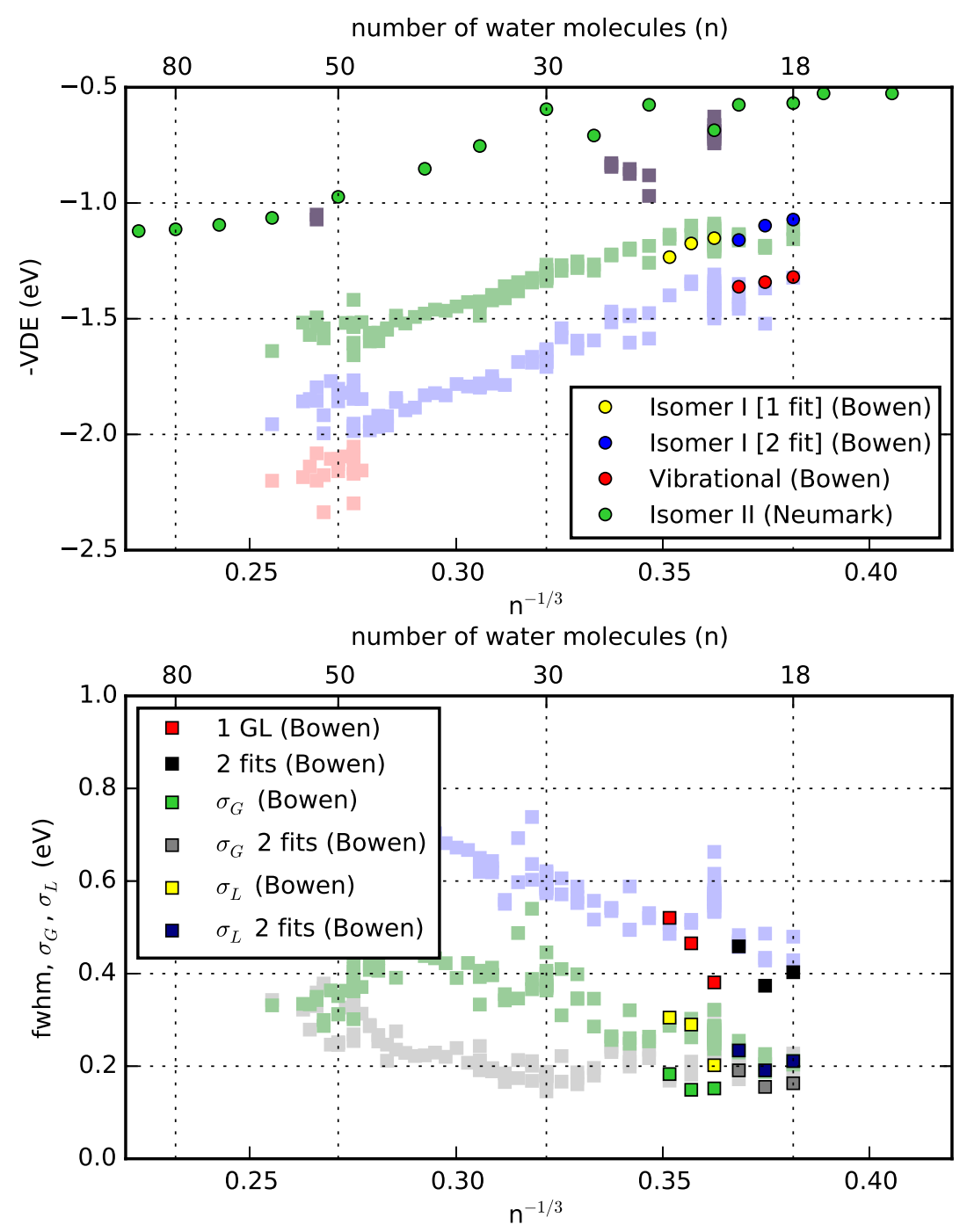

Figure 5.9.: VDEs (upper panel) and peak shape parameters (lower panel) published by other groups in comparison with the multi GL fit results.

formed.

The distinguishing mark of the heavy water cluster spectra, their narrower peak width, mainly caused by a smaller $\sigma_{L}$, is also found in external measurements. This is demonstrated in the lower panel of fig. 5.9 , which compares published peak shape parameters with the parameters resulting from our single GL analysis. The agreement is again very good. An exception poses $\left(\mathrm{D}_{2} \mathrm{O}\right)_{21}{ }^{-}$, which is in our single GL analysis much wider, because the pre-peak of isomer II is not considered. The very small sizes 18 to 21 also show very similar values for $\sigma_{G}$ and $\sigma_{L}$. Although $\sigma_{L}$ is still larger than $\sigma_{G}$, which is in contrast to our findings, the values are close to each other, indicating the same tendency to yield a reversed asymmetry in this 
size range. The reversed asymmetry suggests,that more (metastable) low binding energy isomers contribute to the spectra than currently resolved. Consequently this would mean that heavy water shows a stronger ability to stabilize lower binding energy structures than normal water.

The photoelectron measurements on liquid water-jets of heavy water exhibit also a narrower peak width: $0.78(6) \mathrm{eV}$ in contrast to $0.84(5) \mathrm{eV}$ for normal water[35]. The same tendency can be found in the absorption measurements of bulk water[76]. The values of the peak shape parameters, $\sigma_{G} \approx 0.30 \mathrm{eV}$ and $\sigma_{L} \approx 0.45 \mathrm{eV}[18]$ show that the reduced width $f w h m \approx 0.8 \mathrm{eV}$ originates again from a smaller $\sigma_{L}$. 


\section{Conclusions}

If we interpret the VDEs of isomer I as weighted average of isomers Ia and Ib, basically all available experimental results yield on the whole a consistent picture: the two low binding energy isomers II and III (as named in [77]) are metastable isomers, which exist only for very cold clusters. They need special source conditions and experimental circumstances to be detectable at all. Isomers Ia and Ib respectively are stable isomers, which exist for all experimentally accessible cluster temperatures. The discrepancies of the experimental VDEs can be explained by differences in cluster productions and experimental setup, which leads to different temperatures and temperature distribution of the investigated cluster packages. Especially cold clusters may form many metastable isomers, which blur the borders between the stable isomers.

The high binding energy peak/shoulder (HE), found more or less explicitly for sizes between 45 and 65, is most likely of vibrational origin. First of all it does not show any independent variation in intensity, let alone indications that it evolves into a dominant peak at any cluster size. Secondly, the comparison of its energy offsets to the dominant peak with the energy offsets of deuterated clusters also support this assignment.

Similar considerations apply to peak $\mathrm{Ib}$ for sizes $<33$. For this size range one has to assume that peak Ib is mainly, and in the transition size region at least partially, of vibrational origin.

Discrepancies and inconsistencies in the results of the peak analysis suggest, however, that this picture is not complete. There are hints that besides the dominant and more or less clearly identifiable isomer classes, more (metastable) isomer classes contribute to the spectra. The inconsistent size dependent evolution of the peak shape parameters, even when using 3 GL functions in the peak analysis, strongly points in that direction. This would mean that even in our setup, despite the additional annealing step, there are probably more isomeric contributions in the experimental spectra than the ones located in peak analysis. Experimental setups where the clusters remain cold throughout the experiment, are more likely to produce metastable structures and keep them kinetically trapped on the time scale of the experiment. This is supported by several theoretical calculations, which have highlighted the fact that the start geometries and relaxation conditions strongly influence the resulting cluster structures [36, 59].

Which conclusions can be made about the structure of the isomers, that is, the localization of the excess electron? First of all it should be highlighted that the size 
dependent evolution of the VDEs is compartmentalized for cluster sizes up to 80 molecules, in the sense that the slope changes for certain size intervals. Although the actual size where such slope changes take place depends on the method of peak analysis (i.e. number GL functions), the fact that such changes occur is a common result of all methods. Breaks and shifts in the VDE development motif are expression of structural changes. So is the strongly varying structural stability of clusters in the size range 50 to 60 , visible in the mass abundance spectrum, reflected in the changing peak shapes of photoelectron spectra and fluctuating VDEs. The changes in slope make extrapolations of a certain structure beyond the switching point questionable.

The electron binding motif of isomer I(a) for small sizes $(\leq 7)$ could be identified by a combination of infrared spectroscopy and ab initio calculations [26, 27, 29, 62], as a "double acceptor" or AA motif, in which a water molecule that accepts two hydrogen bonds and donates none, binds the electron directly. Although subsequent experiments [28, 61] suggest that the AA motif may be found for cluster sizes up to the mid 20s, it is questionable to derive a surface localization of isomer Ia for larger sizes, due to the discussed structural changes and growth motifs. A comparison with the results of theoretical simulations revealed, however, that the VDEs of surface isomers calculated by Barnett et al. and Jacobson and Herbert are in good agreement with isomer Ia for the entire size range. Together with its size dependent abundance, i.e. dominant for small sizes and decreasing from size 46, this seems a plausible assignment.

Isomer $\mathrm{Ib}$ appears in the experimental spectra at approximately $n=35$ and becomes dominant for sizes with more than 46 water molecules. The calculations of Barnett et al. correlate it with an isomer with interior electron localization [5, 6] starting at size 55. In the calculations of Jacobson and Herbert the VDEs of isomer Ib fit best with the partially embedded cavity isomer at size 40, lie in between of the partially embedded and interior cavity up to size 100, and are in excellent agreement with the interior cavity isomer for size 200 [36]. This suggests that isomer Ib starts with a structure which forms a partially embedded cavity to solve the excess electron. Around size 50 begins a stronger internalization of the cavity or even a restructuring to form the interior cavity (remember that all experimental data indicate strong structural changes in this particular size range). Somewhere around size 100 the restructuring/internalization process comes to an end and a constant growth motif with the cluster size starts.

These assignments are, however, questioned by calculations by Turi [73, 175]. Although there is now agreement that the interior localized electron is indeed solvated in cavity structure[72] (as opposed to a non-cavity interior localization), the calculated VDEs for surface localized electron isomers are a better match [73].

The metastable isomer II shows also a moderate size dependent development of the VDEs. Starting with fluctuations in the size range 20 to 30 and a distinct step between 30 and 50. For sizes large than 50 the VDEs increase slowly but linearly 
with the cluster radius. Barnett et al. assign them to isomers where the excess electron is diffusely localized on the cluster surface[6].

Isomer III was only found for deuterated clusters starting at size 11[17, 77]. The VDEs connect, however, nicely to measurements of small water clusters $\leq 11[43$. Since the VDEs of isomer III show almost no size dependency, they are loosely attached electrons. Jacobson and Herbert assigned them to dipole bound surface isomers.

The theoretical results, with few exceptions, fit on the whole nicely to the experimental results. They encourage the assignment of isomer Ia to a surface state isomer, even for medium sized clusters consisting of up to 100 water molecules. Interior state isomers exist clearly for sizes beyond 80. The internalization process, however, starts already for smaller sizes, possibly by an intermediate structure of a partially embedded cavity around size 40 . In fact the strong structural variations in the size range between 50 and 60 may indicate sizes, where the formation of an fully interior cavity becomes geometrically and energetically possible. Isomer Ib represents therefore the interior state isomer, with full internalization completed around size 80 .

What follows then for the extrapolation to bulk hydrated electron? The very early conclusion[16] that isomer I develops into the bulk value remains essentially correct. For an accurate linear extrapolation it is necessary, however, that the growth motif must conform with the dielectric sphere model. This means that the solvation structure of the electron must remain the same with increasing cluster size and only the cluster radius grows. Independent of the peak analysis and the number of fit functions the experimental results suggest that this is only fulfilled for clusters with more than 80 water molecules. Most theoretical calculation support this assessment by placing a full internalization in the same size range. Extrapolations including data from smaller cluster sizes yield results, which in the end depend on the size range used for the linear fit.

The extrapolation of our data by a linear fit starting at size 80 places the bulk value of the hydrated electron at $3.60(3) \mathrm{eV}$. In the discussion we have already seen that this is in excellent agreement with the results of one liquid water-jet experiment, while the others are as far as $0.3 \mathrm{eV}$ lower.

When comparing the experimental results of water clusters and liquid water-jets, we implicitly assume that effects which may occur due to the temperature difference of nearly $200 \mathrm{~K}$ are negligible. In the end this means, we assume that the solvation structure of the excess electron is in both systems the same. There are several hints, which make this approximation questionable. First there is the recurring question, if experimentally produced clusters assume a fully relaxed structure or structures. The analysis of the peak parameter suggests that depending on the experimental setup, metastable, kinetically trapped structures contribute at least partially to the photoelectron line shape. Calculations of the dynamics of the electron localization in warm and cold water clusters[59] come to the result that most likely higher 
temperatures than the experimentally accessible ones are necessary to exclude the formation of such metastable isomers.

But even if we assume that the spectrum of isomer Ib originates mainly from relaxed structures, there is still the matter of the phase. Is it a valid approximation to compare the extrapolations of VDEs of solid (or semi-solid) water clusters to VDEs of liquid water-jets? If we apply the dielectric sphere model to solid water ice, it predicts a different size dependency (slope) due its relation to the relative permittivities. Especially the relative static permittivity of water varies strongly with the temperature and the structure (liquid, crystalline ice, amorphous ice).

Fig. 6.1 brings the predictions of the dielectric sphere model (eq. (3.2p) for different slopes and VDEs in relation to the cluster VDEs. The chosen bulk VDEs

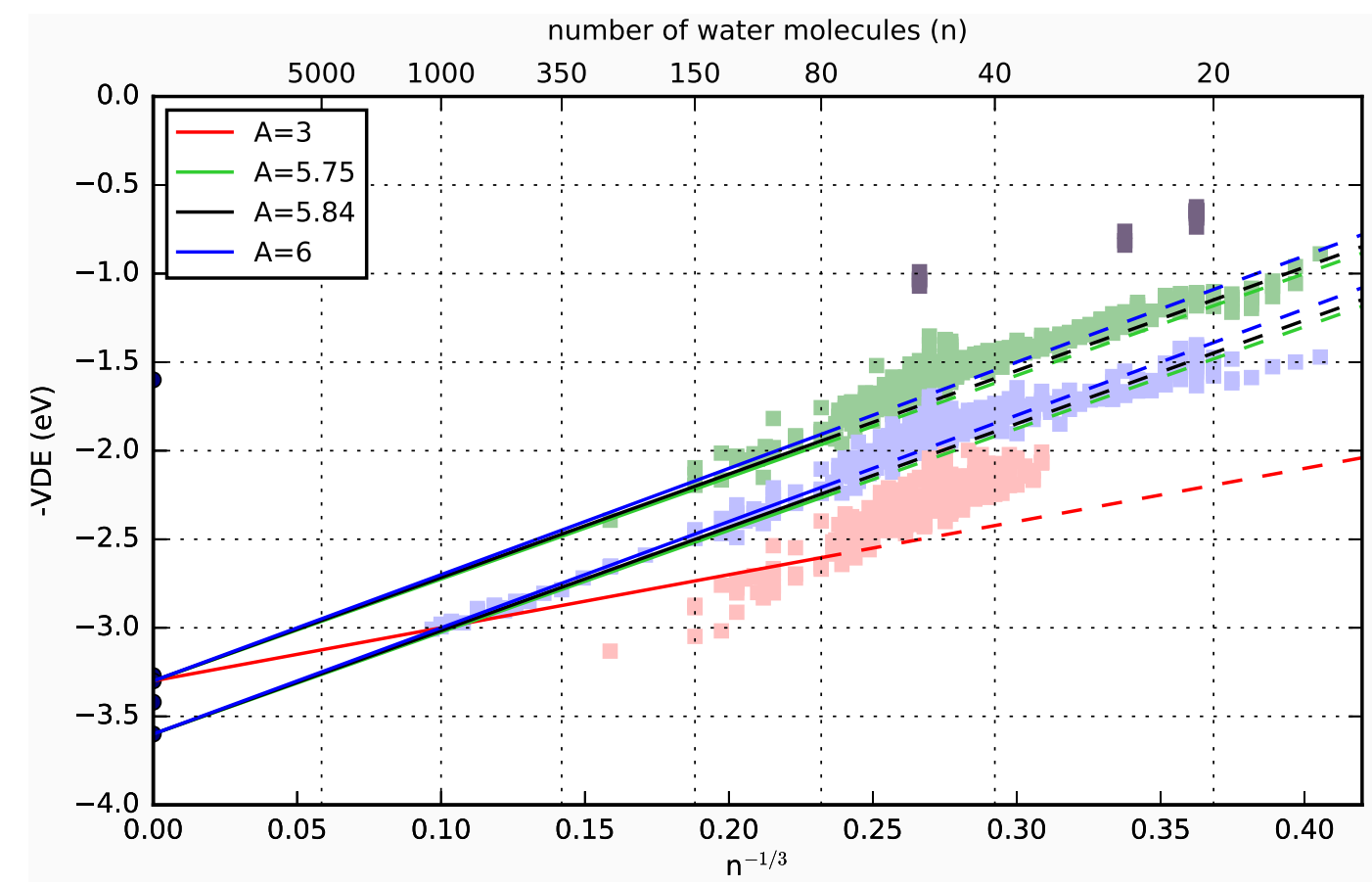

Figure 6.1.: Prediction of the dielectric sphere model for various slope values when starting at two different bulk VDEs.

represent the two outermost values of the experimental water-jet results, $3.3 \mathrm{eV}$ and $3.6 \mathrm{eV}$. The red slope indicates the hypothetical case, which connects the VDE of $\left(\mathrm{H}_{2} \mathrm{O}\right)_{1100}{ }^{-}$with the lowest bulk value of $3.3 \mathrm{eV}$. If one assumes the extrapolation of the cluster data to the bulk as valid approximation, such a size dependent development of the VDEs for larger cluster sizes seems very unlikely. This is supported, though not excluded, by the very narrow value range of the permittivities $\sigma_{r, \nu}$ and $\sigma_{r, s}$ for this slope value.

The blue and green slopes correspond to the experimentally determined values for 
multi and single GL analysis respectively. Naturally, they fit very well to the isomer Ib VDEs, when starting at $3.6 \mathrm{eV}$. Assuming a bulk value of $3.3 \mathrm{eV}$, they seem to correlate with the isomer Ia data. However, there are currently no indications that this is more than pure chance.

The most interesting slopes are the black ones, which conform with the liquid water permittivities at $300 \mathrm{~K}$. The slope value lies between the experimentally found values. This means that the predicted VDEs coincide within the accuracy of our analysis with the measured cluster VDEs, when starting at $3.6 \mathrm{eV}$. Consequently deviate the VDEs of the dominant cluster isomer Ib by about $0.3 \mathrm{eV}$, when we choose $3.3 \mathrm{eV}$ as bulk value.

Given the excellent agreement of the large cluster VDEs with the linear size dependency demanded by the dielectric sphere model, one can expect the extrapolation to yield a valid prediction of the progression of the VDEs for even larger sizes. The extrapolated bulk value supports therefore strongly the liquid water-jet results with higher binding energies. Especially since the slope value for liquid water corresponds so well with the ones found for (solid) clusters.

However, one should bear in mind that the extrapolated bulk value gained from cluster experiments, stands for the VDE of a hypothetical system, which conforms with the properties large water clusters assume. Equating the extrapolation value with the VDE of liquid water is therefore only valid, if these properties, e.g. the solvation structure of the excess electron, the permittivities and the density or the intermolecular distances of the water, are very similar in both systems. Due to the strong correlation between the temperature and the permittivities and density of water, this is a priori not necessarily the case. A final conclusion about the VDE of the hydrated electron in bulk water can therefore not be drawn from the current cluster measurements, but would require further investigations of the structural similarities or differences between water clusters and bulk water. 



\section{Summary and Outlook}

Photoelectron spectra of water cluster anions have been recorded for a large size range, extending the known sizes to $\left(\mathrm{H}_{2} \mathrm{O}\right)_{1100}{ }^{-}$. A detailed peak analysis has been conducted using single or multiple Gaussian-Lorentzian functions to fit the experimental spectra. It revealed that the spectra is constituted predominantly by the contributions of several peaks, which often overlap to form the typical broad and asymmetric line shape. Due to their (Gaussian) widths, the peaks Ia, Ib, and II represent rather distinct isomer classes than single isomers. Peak HE originates presumably from vibrational excitement. Discontinuities in the size dependent evolution of the peak width parameters of the GL functions suggest, however, that even more isomers contribute to the spectra than the ones captured by our analysis.

The values of the ascertained VDEs of each isomer class depends to a certain degree on the number of GL functions used for the fit. For many sizes this number can currently not be determined unambiguously from the experimental data, thus introducing a small uncertainty in respect of the values of the VDEs. Nevertheless a general trend of the development of the isomer classes has been found. The stable lower binding energy isomer class Ia is dominant for small cluster sizes consisting of up to 46 water molecules. For all larger sizes isomer Ib becomes the dominant isomer. No higher binding energy isomers has been found in the spectra, not even for the very large cluster sizes. The metastable Isomer II could be detected for few sizes only and requires a cluster temperature below $120 \mathrm{~K}$.

The analysis of temperature dependent measurements revealed changes in the line shape of the photoelectron spectra for temperatures below and above the melting point of the clusters. In the more detailed analysis of the sizes around $\left(\mathrm{H}_{2} \mathrm{O}\right)_{48}{ }^{-}$, alteration of the spectra have been found, reflecting the three "phases" found in caloric measurements: solid, melting and evaporative.

The VDEs of small and medium sized clusters exhibit a piecewise linear evolution with the inverse cluster radius. Only the larger clusters, starting at size 80, develop a continuous, linear size dependency of the VDEs of isomer Ib, indicating a constant structural growth. As a consequence only VDEs of sizes larger 80 were used to linearly extrapolate the VDE of isomer Ib, yielding a bulk value of $3.60(3) \mathrm{eV}$. The size dependency of these large clusters, resulting from the extrapolation, shows a remarkable similarity with the prediction of the dielectric sphere model, when using permittivities and density of liquid water.

The results of our measurements have been compared to the findings published by other groups. The experimental VDEs show in general a good agreement, but 
diverge from each other for medium sized clusters. This is caused by our use of multiple GL function for fitting the spectra, which results in two stable isomers instead of one. However, the analysis of the width parameters revealed that both isomers are probably present in all measurements, but could not be resolved due to differences in the experimental setups. When factoring in this assumption, the agreement of the experimental results is excellent. The extrapolation to the bulk coincides best with the photoelectron measurements of liquid water-jets resulting in VDEs with higher binding energies.

The calculated VDEs scatter more broadly, which might not come as a surprise, given the high number of isomers. Most calculations support, however, the assignment of isomer Ia to structures with surface localized excess electron and isomer Ib to structures with an interior localized, cavity solvated excess electron. A complete internalization is reached for sizes between 65 and 100, which coincides well with the constant structural growth scheme we have found to start around 80 . Thus the medium sized isomer Ib clusters refer probably to structures with a partial electron internalization.

For the small sizes, peak Ib seems to originate rather from vibrational excitement than from a distinct isomer. The same applies to the high binding energy peak HE of the medium sized clusters. This assignment is supported by differences in peak distances found in the spectra of normal and deuterated water clusters.

In a complimentary investigation, the photoelectron spectra of deuterated water cluster anions have been measured, covering the sizes 18 to 60. It has been found that the VDEs of heavy water clusters are basically identical with the VDEs of their non-deuterated counterparts. The peak shape analysis revealed, however, that the widths are considerable narrower, mainly due to the lower width of the high binding energy side of the peak.

\section{Outlook}

One of the largest uncertainty concerning the analysis of experimental photoelectron spectra arises from its unknown composition, or, to put it differently, from the unknown number and shape of the fit functions to map the spectra. A better knowledge of the underlying substructures would therefore further improve the determination of the VDEs of each isomer and their temperature dependent development. This becomes essential, when comparing experimental findings quantitatively with theoretical calculations. The high number of possible isomers makes an assignment of cluster structures based on the VDEs a difficult endeavor.

One method to probe the underlying substructures and isomers is the hole burning technique. In a hole burning experiment a lower binding energy isomer is selectively photodetached by irradiating the clusters with an additional laser with appropriated photon energy prior to the actual photodetachment for the photoelectron spectroscopy. If the main peak were composed of only few distinct isomers, 
hole burning would therefore allow to remove one single isomer from the spectra. By calculating difference spectra, the spectra of each isomer could be reconstructed. Currently we assume that the substructures are comprised rather by isomer classes than single isomers. Hole burning experiments could clarify this aspect. Even in the case of isomer classes, hole burning in the energy range of Gaussian slope, could provide further insight into the composition of individual isomer contributions. In combination with temperature dependent measurements, it may also be possible to investigate the rearrangement of isomers on the time scale of the experiment.

Photoelectron spectroscopy of methanol cluster anions pose another interesting and complementary measurement. Methanol $\left(\mathrm{CH}_{3} \mathrm{OH}\right)$ is the simplest alcohol and therefore represents the "next" step from the hydrated electron in water to the solvated electron in alcohols. Similar to water, methanol is capable of forming hydrogen bonds due to the polarity of its hydroxy group.

Photoelectron measurements of methanol cluster anions [40] have yielded two isomers, of which the tighter bound isomer I could represent an interior solvation structure of the excess electron, similar to isomer Ib of the large water clusters. The extrapolation of the isomers I VDEs to the bulk resulted, however, in a significantly lower value $(2.6 \mathrm{eV})$ than the one found in micro-jet experiments $(3.38 \mathrm{eV})$ [35, 67]. The discrepancy of the size dependent slope of clusters and the prediction of the dielectric sphere model for liquid methanol[40] may arise from the readiness of methanol to enter a glassy state. This would mean that methanol clusters are more likely to be trapped in metastable structures without extensive annealing. In the light of the differences we have found for water clusters produced in different setups, photoelectron spectroscopy of methanol clusters using our experimental conditions might gain further insight in this matter.

Initial steps to produce methanol clusters have been undertaken. The mass spectra revealed, however, a strong fragmentation of the clusters, probably even on the molecular level. Currently we suspect that the clusters fragment already in the source during the charging process in the plasma of the ring discharge. One possible solutions would be to charge rather the molecules than the clusters, by placing a discharge ring near the inlet. This might, however, destroy the methanol molecules. A more promising solutions would be to charge the clusters by low kinetic energy electrons. These could be produced by irradiating surfaces whose work functions lie slightly below the energy of the UV photons. 



\section{A. Photoelectron Spectra}

\section{A.1. Water Cluster Anions (590 nm)}

Photoelectron spectra of $\left(\mathrm{H}_{2} \mathrm{O}\right)_{\mathrm{n}}{ }^{-}$recorded at $590 \mathrm{~nm}(2.1 \mathrm{eV})$. The intensity is given in arbitrary units. Fits according to the "best compromise" multi GL analysis.
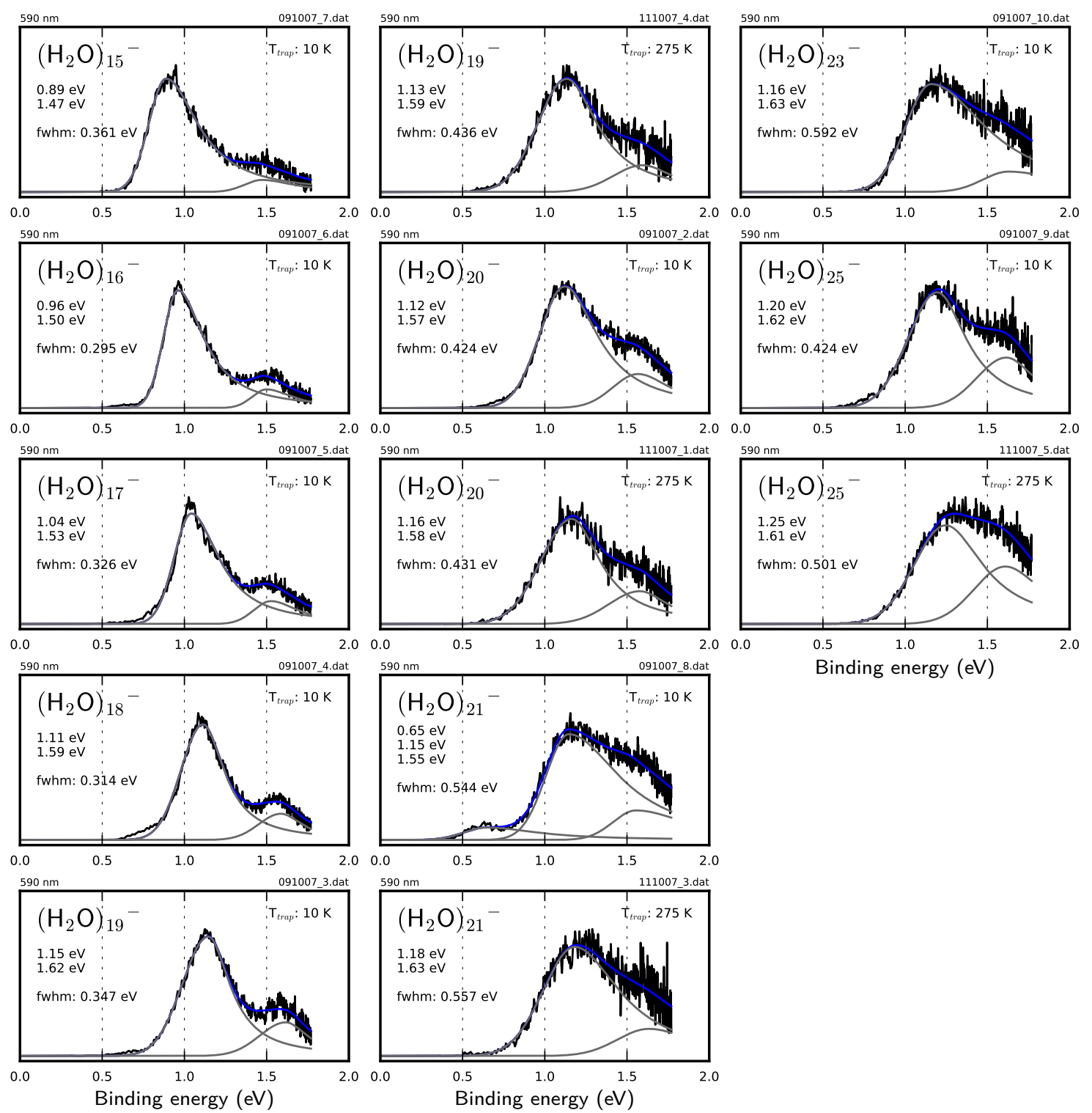


\section{A.2. Water Cluster Anions (248.4 nm)}

Photoelectron spectra of $\left(\mathrm{H}_{2} \mathrm{O}\right)_{\mathrm{n}}{ }^{-}$recorded at a detachment laser wavelength of $248.4 \mathrm{~nm}(4.99 \mathrm{eV})$. The intensity is given in arbitrary units. The fits show the results of the multi GL analysis according to the "best compromise" approach.
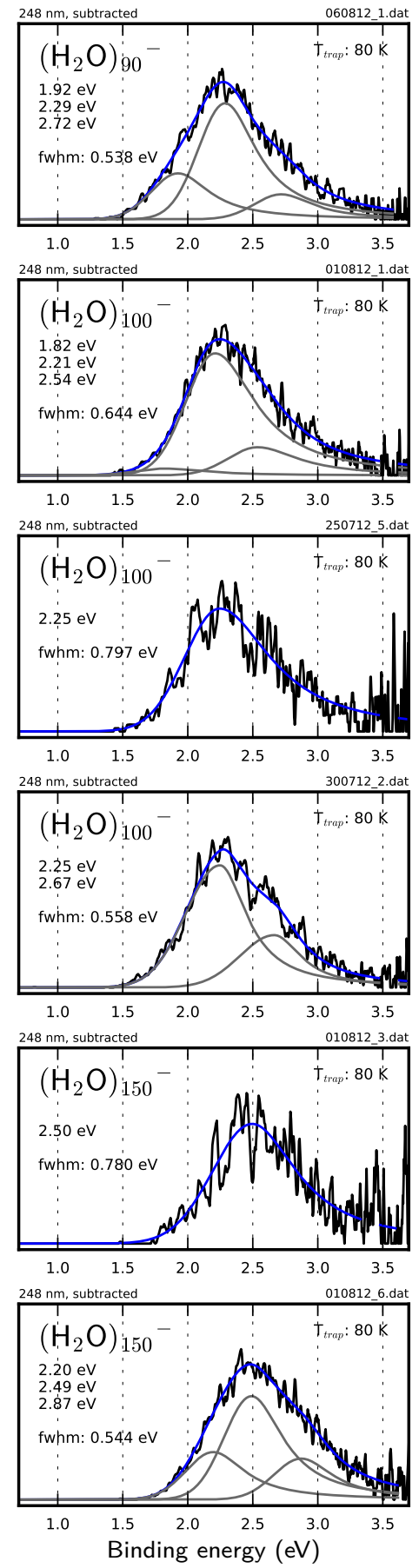


\section{A.3. Water Cluster Anions (308 nm)}

Photoelectron spectra of $\left(\mathrm{H}_{2} \mathrm{O}\right)_{\mathrm{n}}{ }^{-}$recorded at a detachment laser wavelength of $308 \mathrm{~nm}(4.02 \mathrm{eV})$. The intensity is given in arbitrary units. The fits show the results of the multi GL analysis according to the "best compromise" approach.
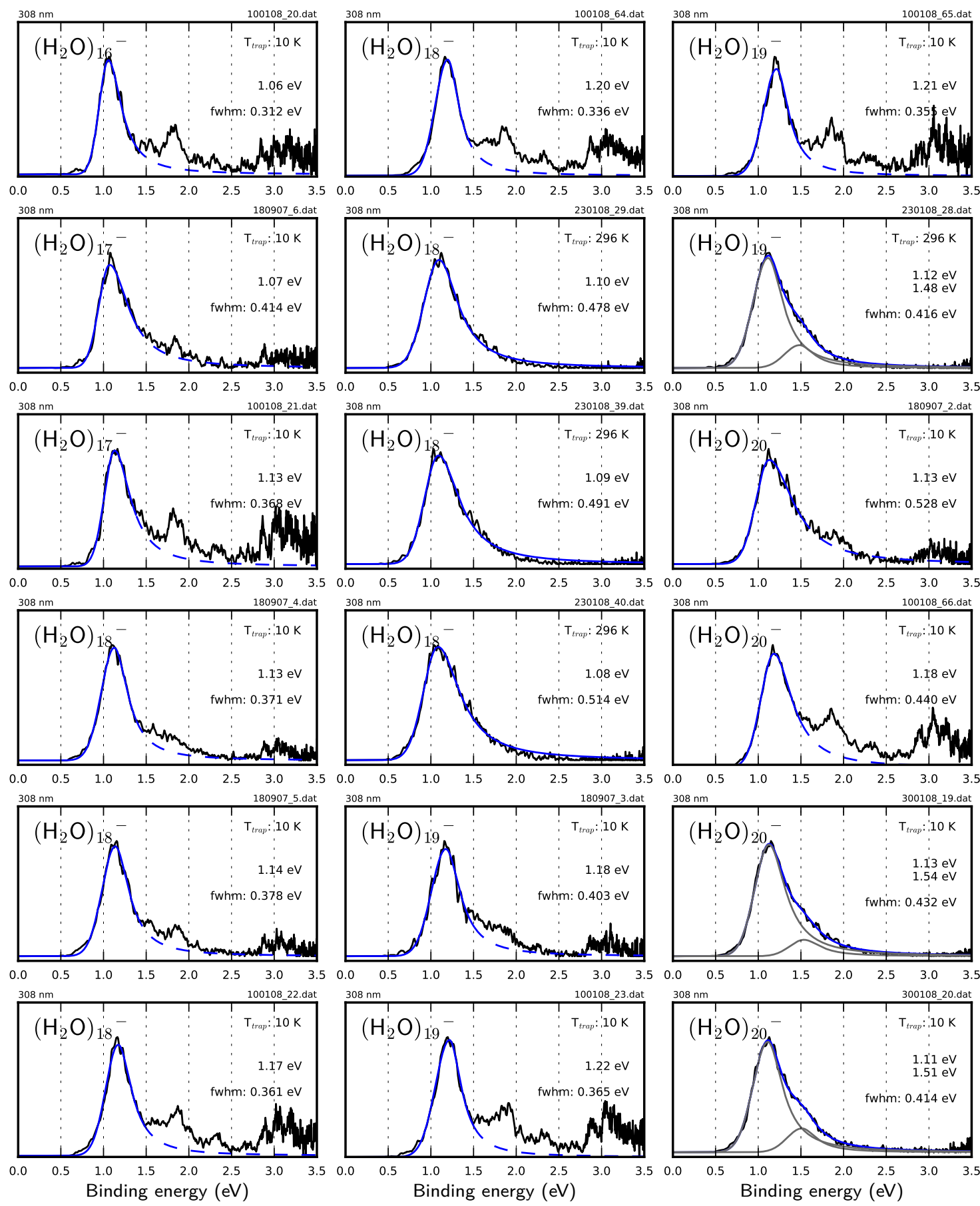

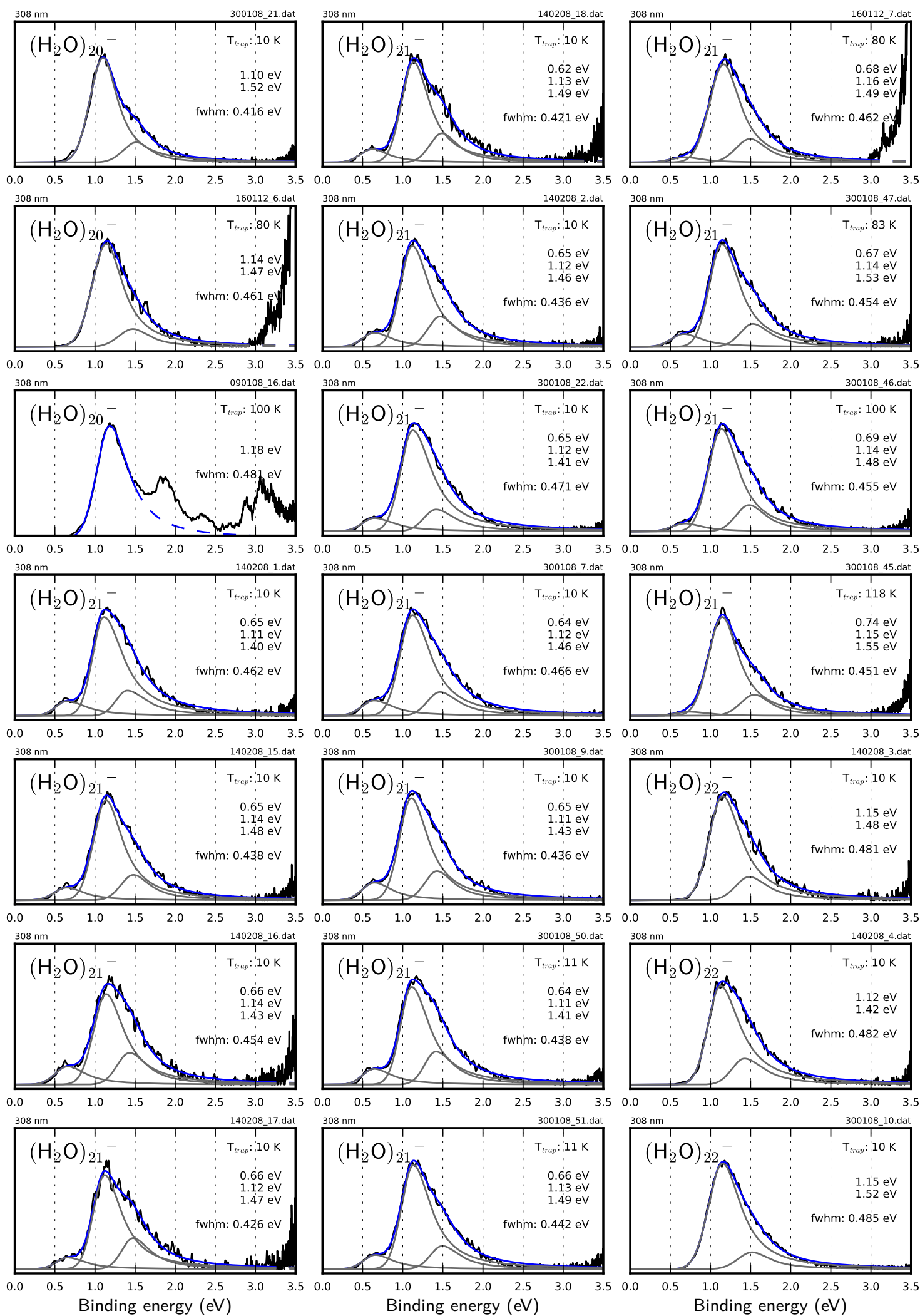
A.3. Water Cluster Anions (308 nm)
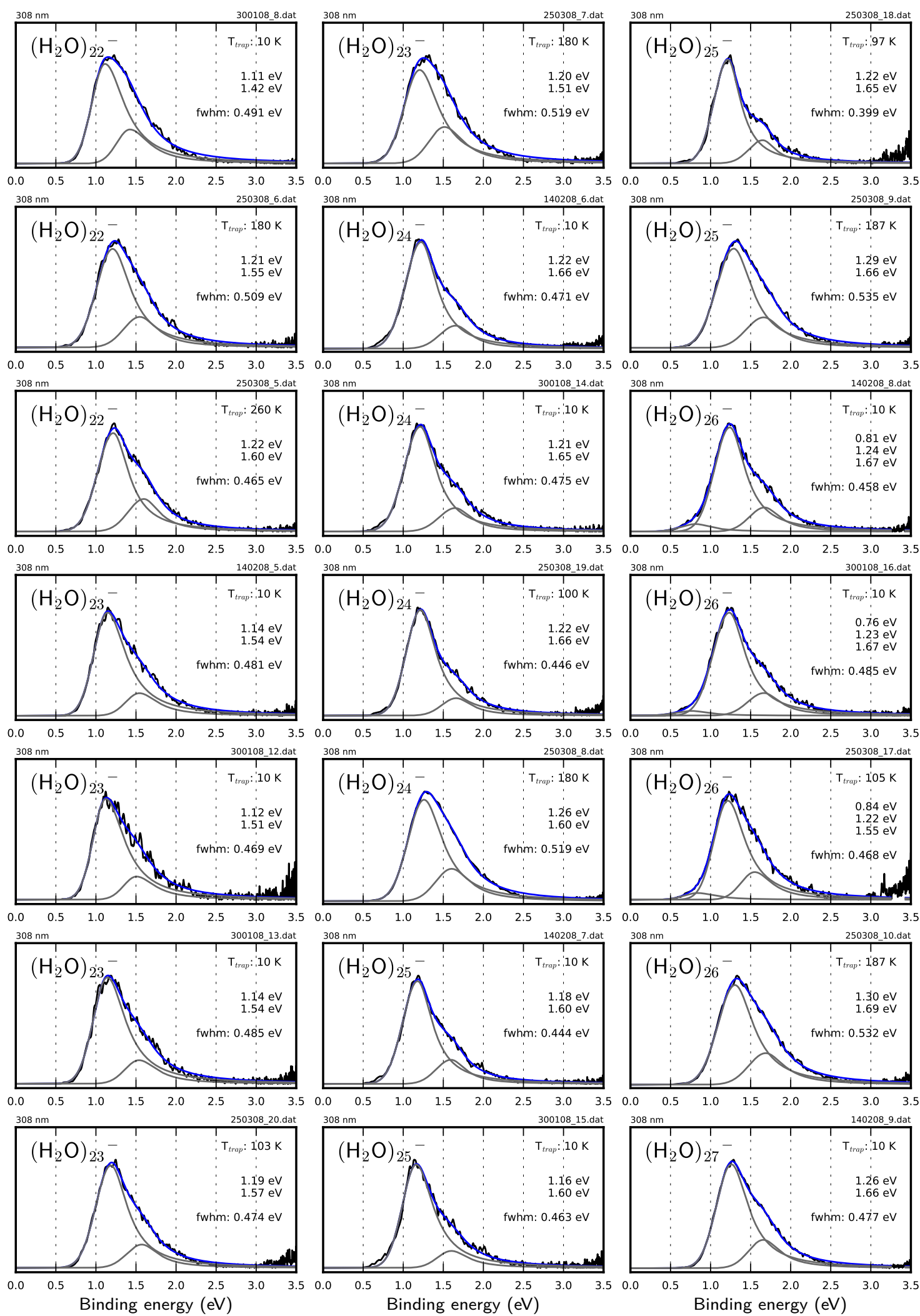

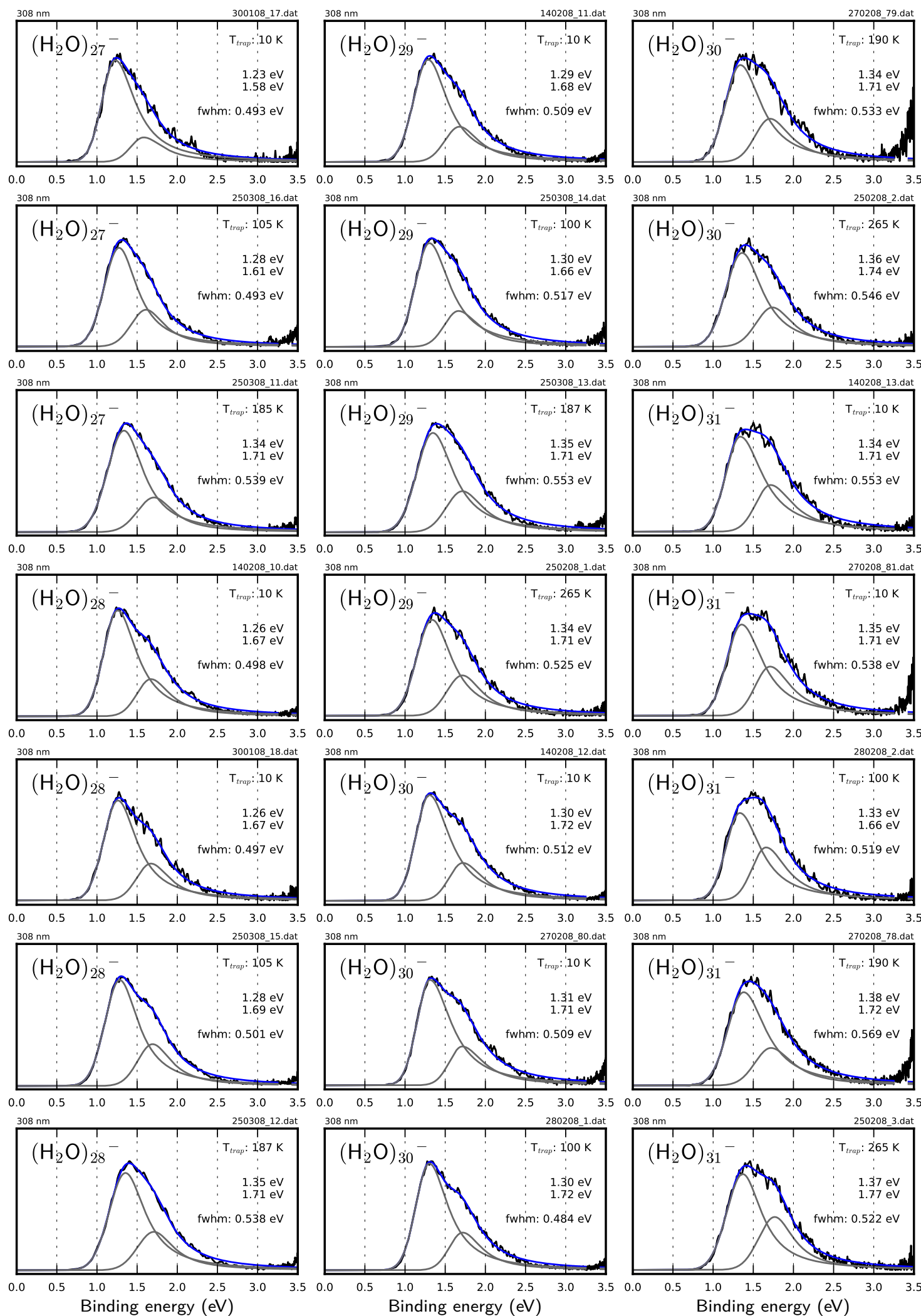
A.3. Water Cluster Anions (308 nm)
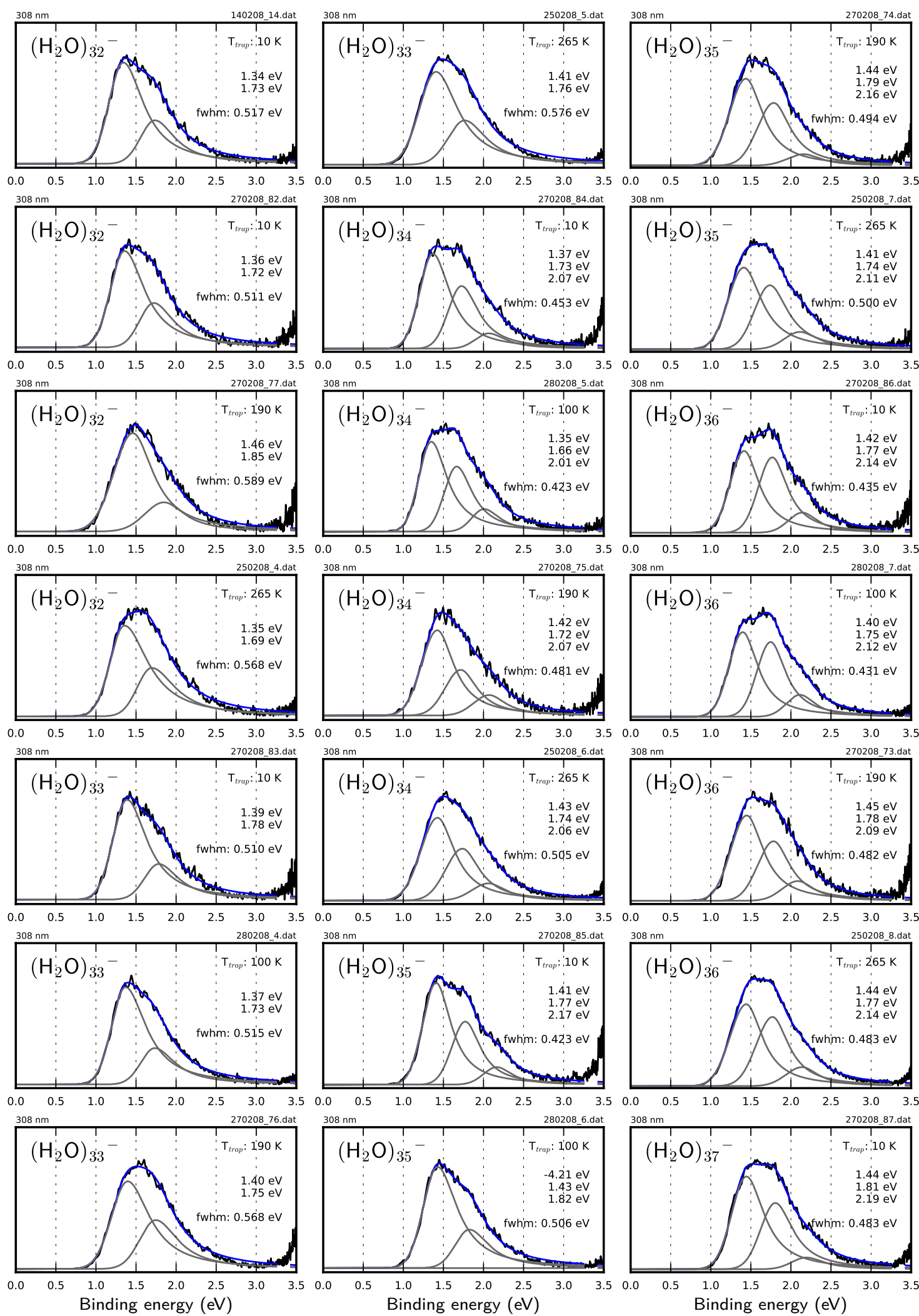

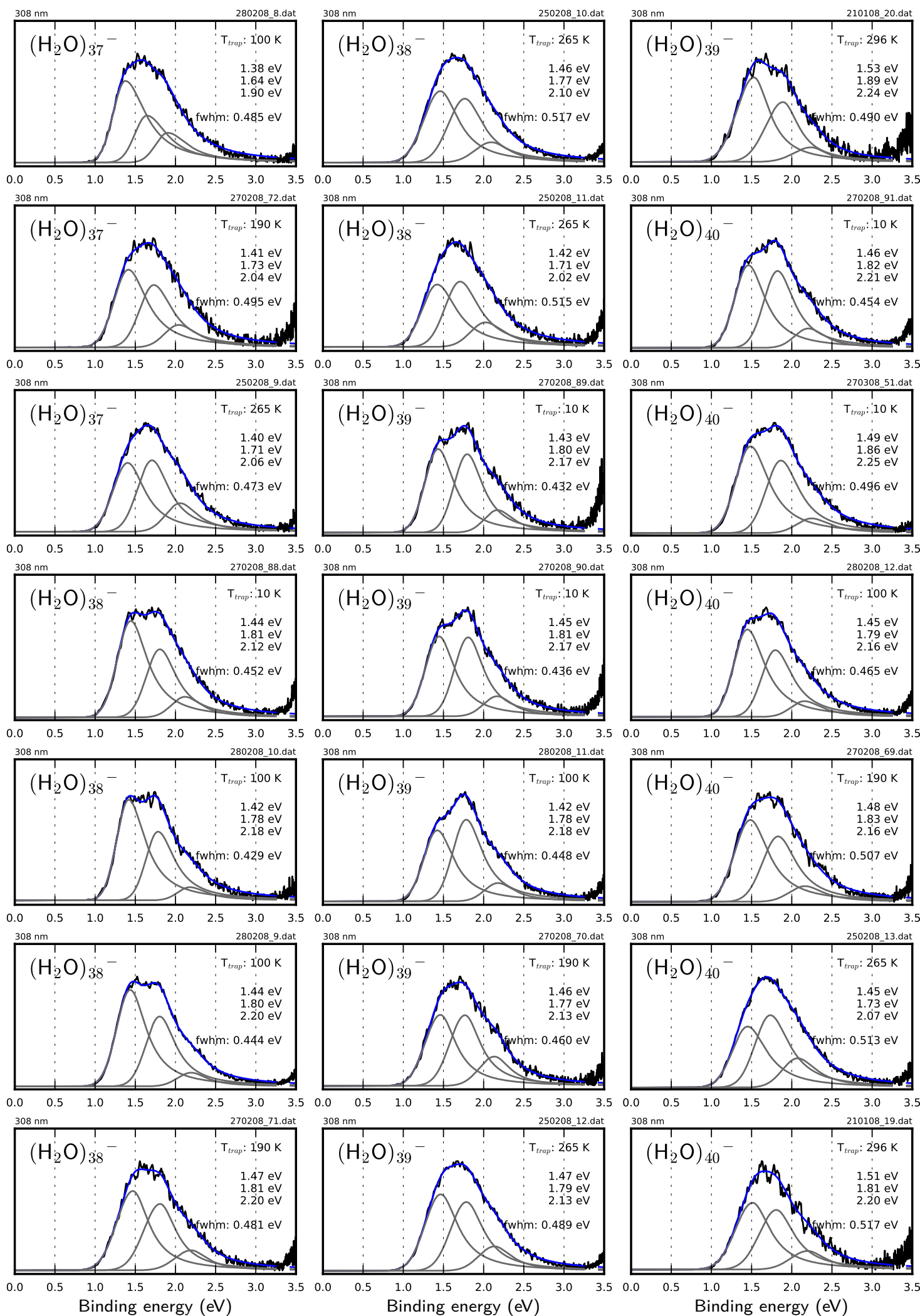
A.3. Water Cluster Anions (308 nm)
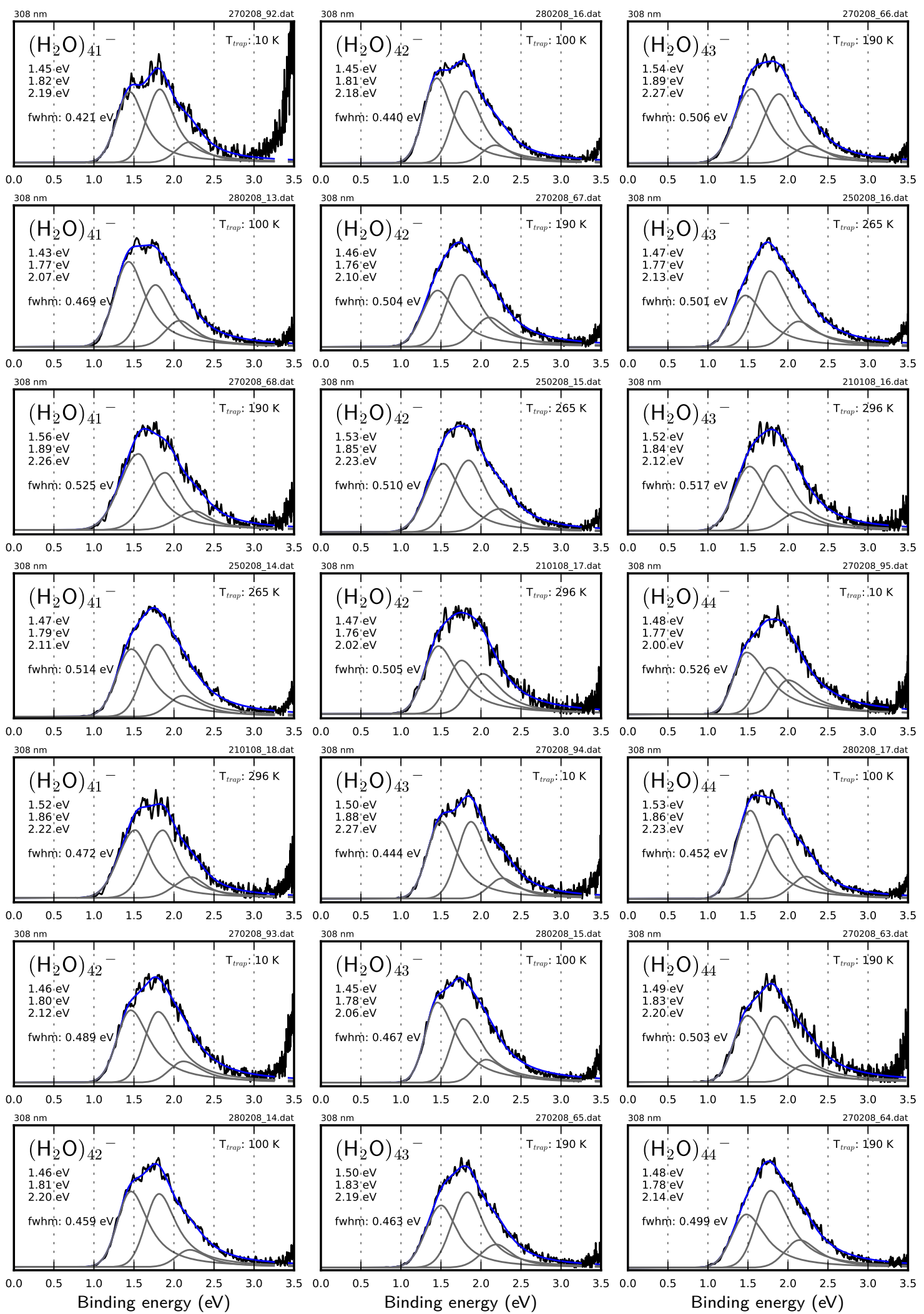

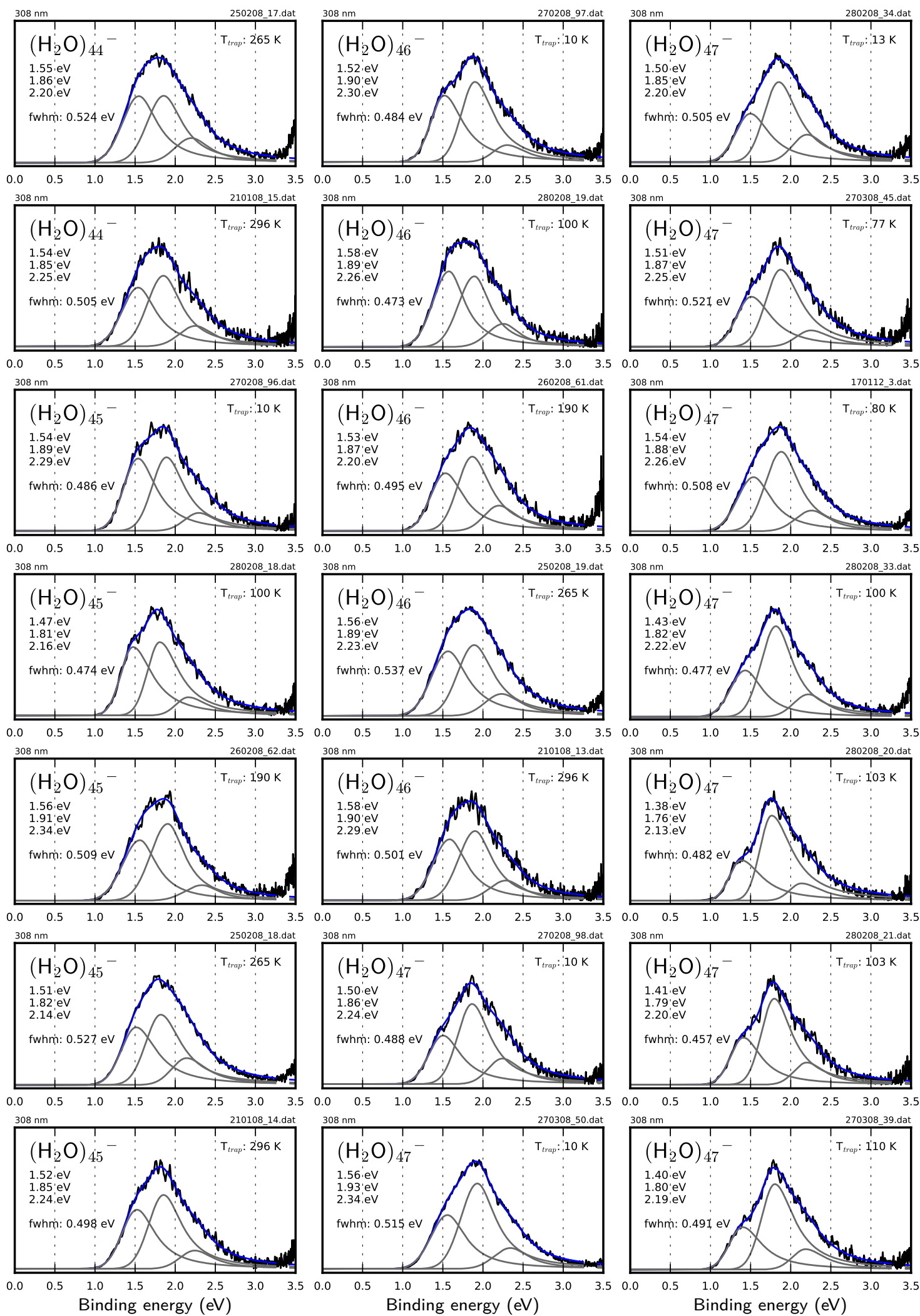
A.3. Water Cluster Anions (308 nm)
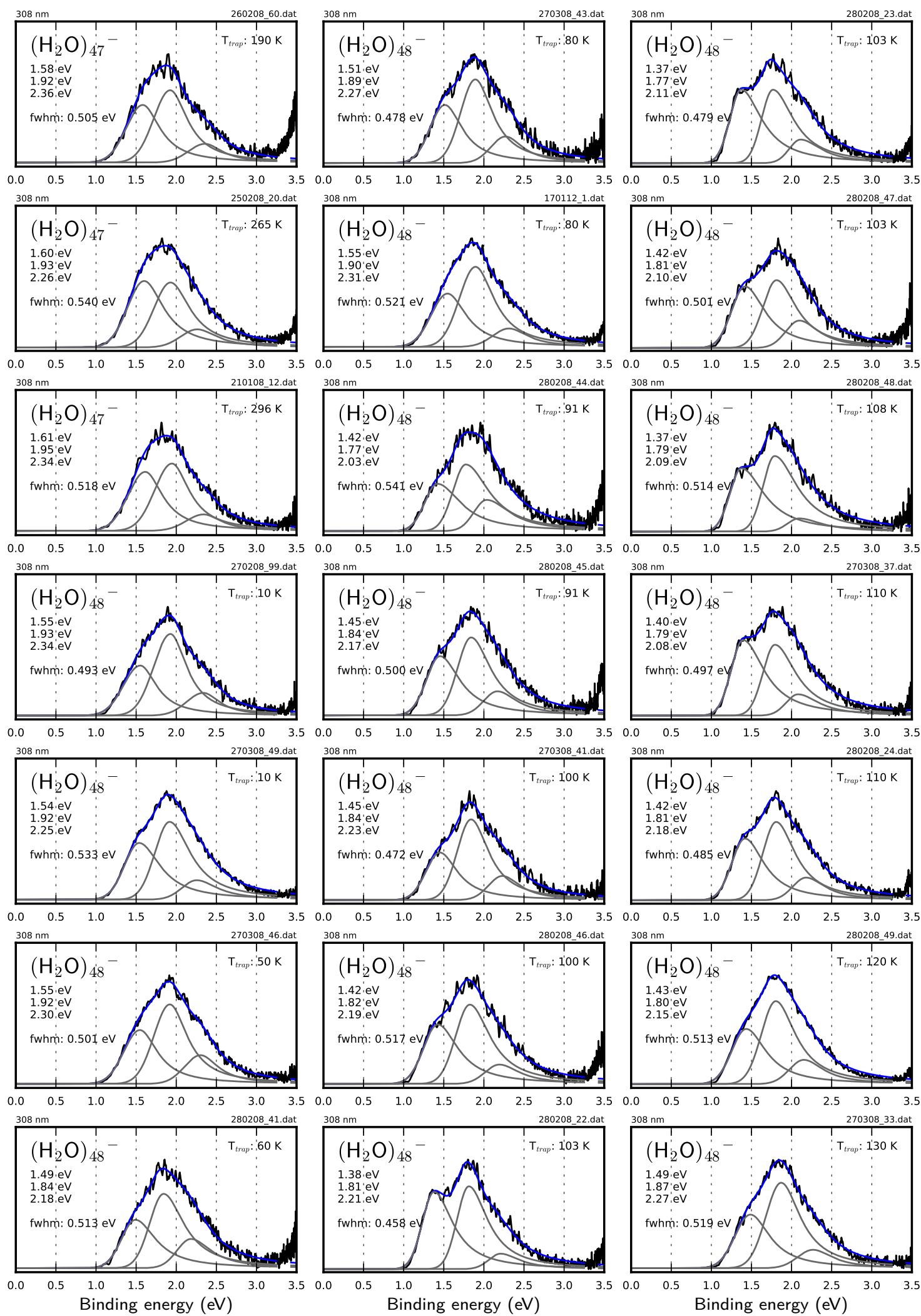

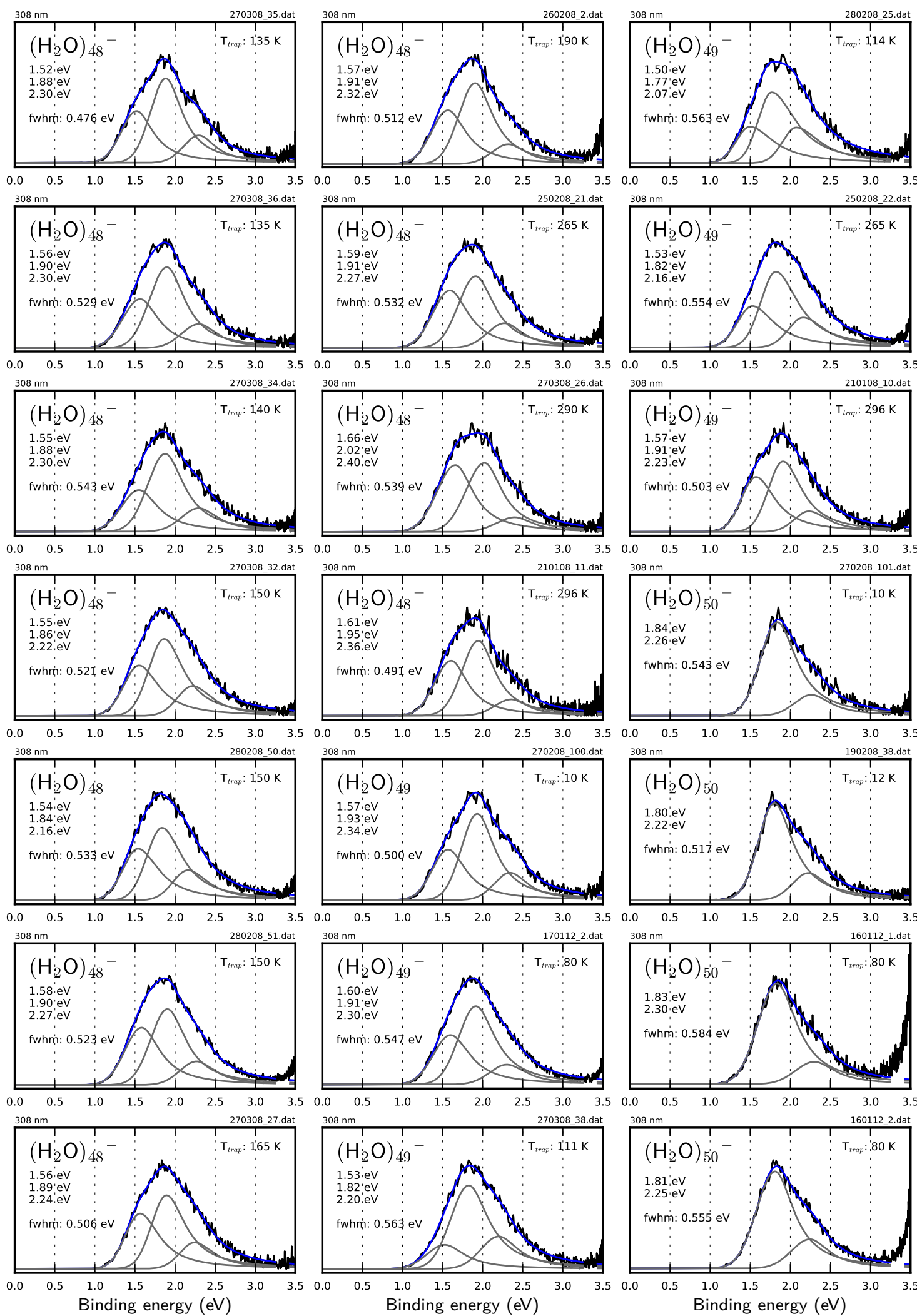
A.3. Water Cluster Anions (308 nm)
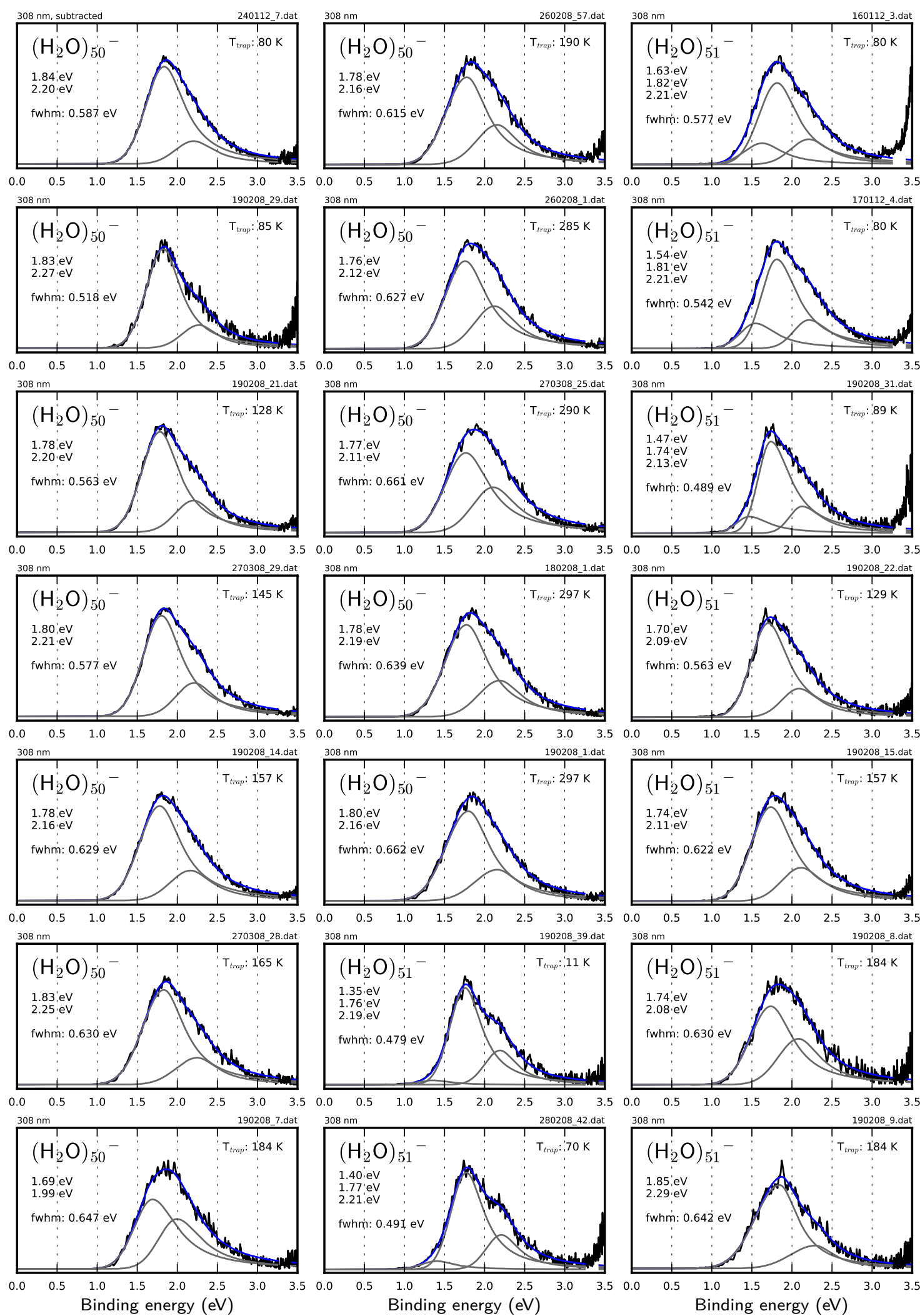

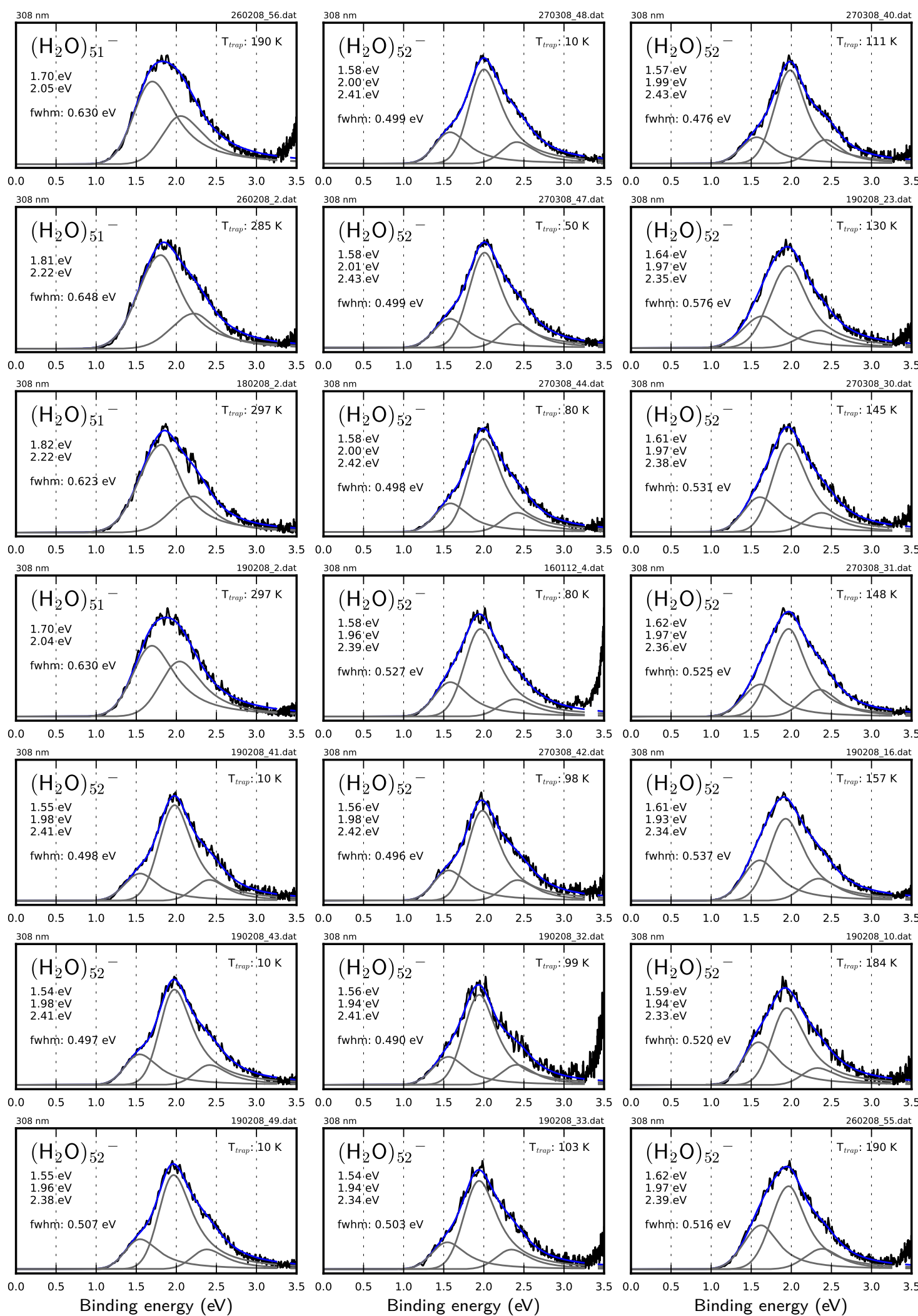
A.3. Water Cluster Anions (308 nm)
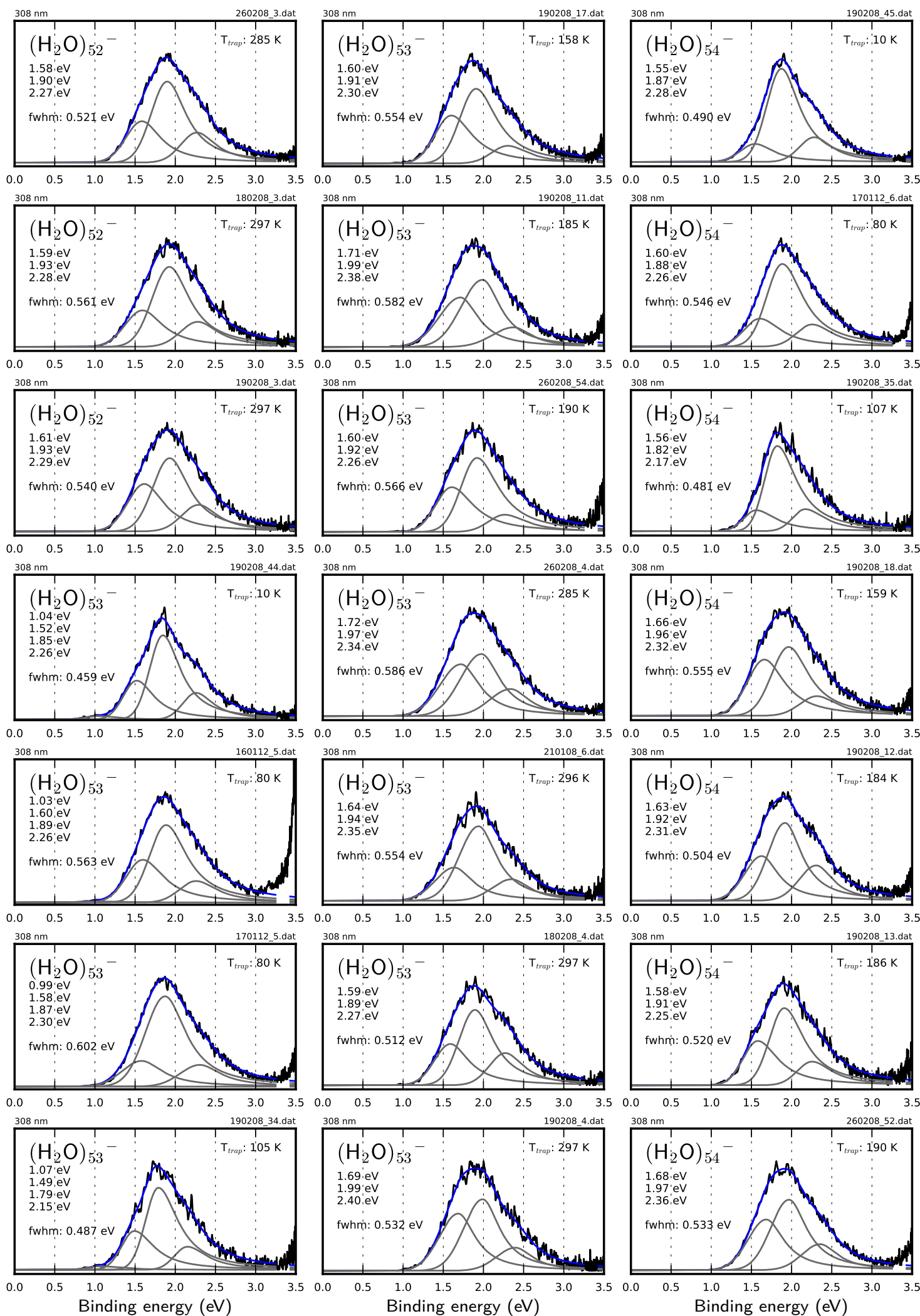

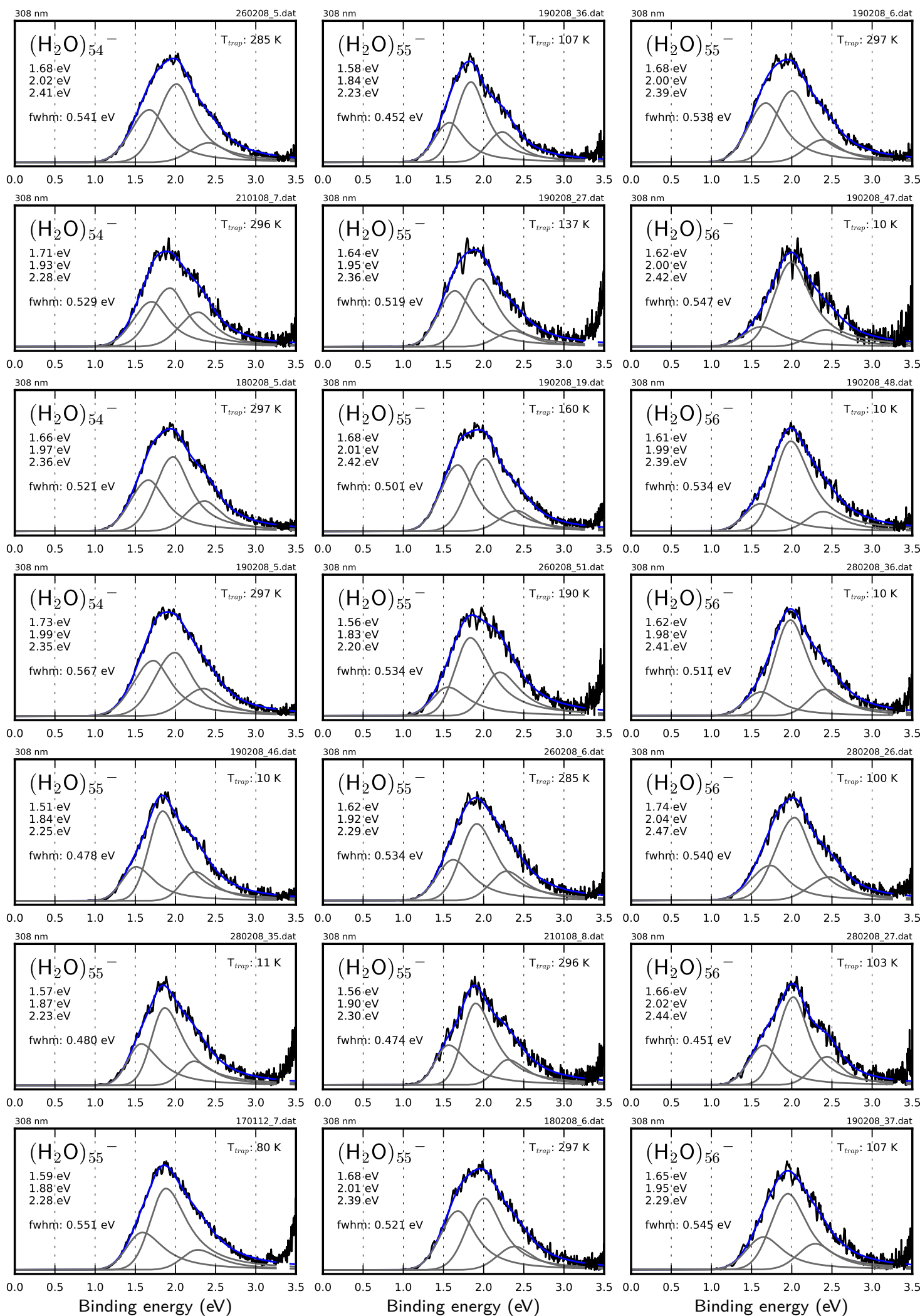
A.3. Water Cluster Anions (308 nm)
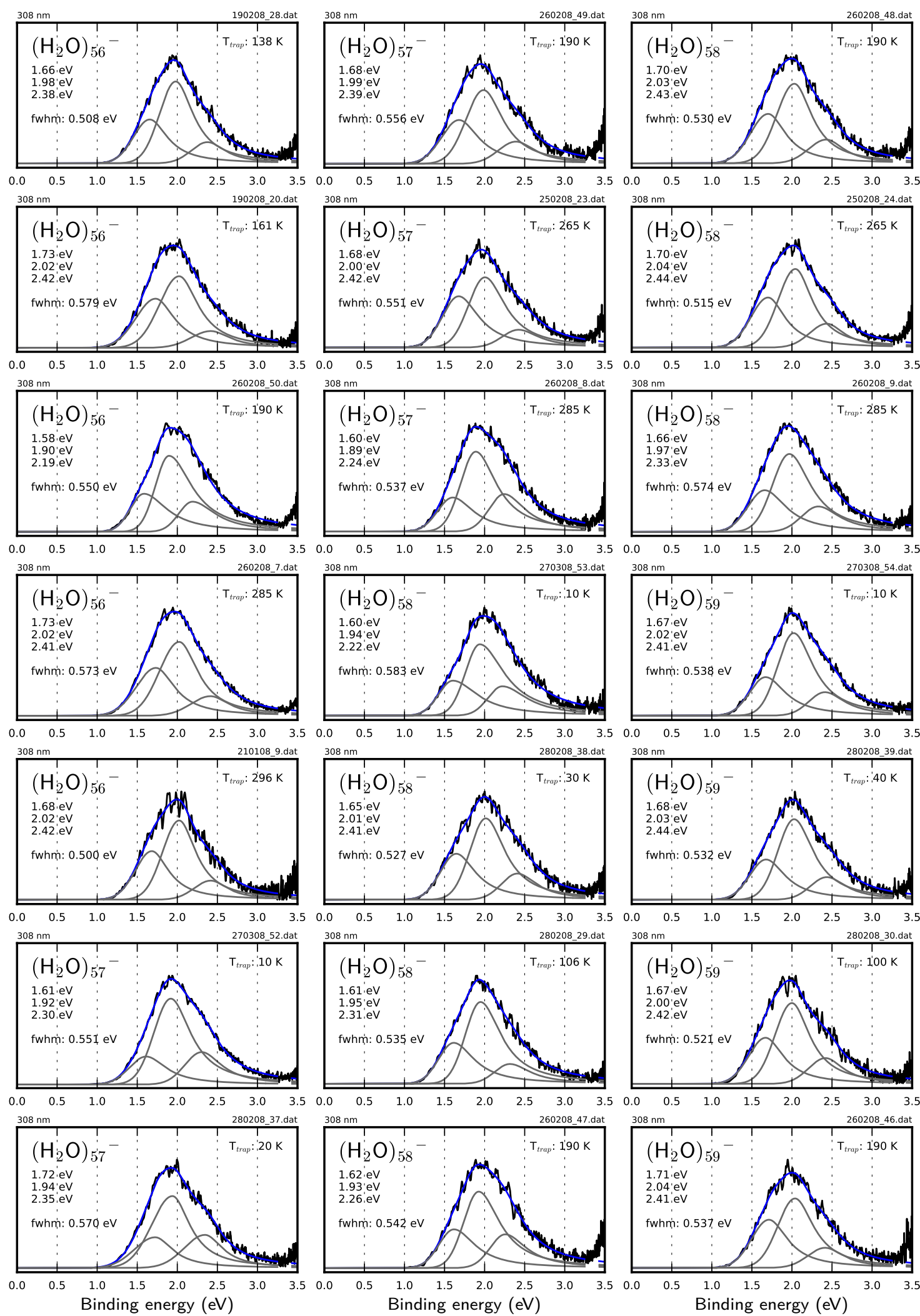
A. Photoelectron Spectra
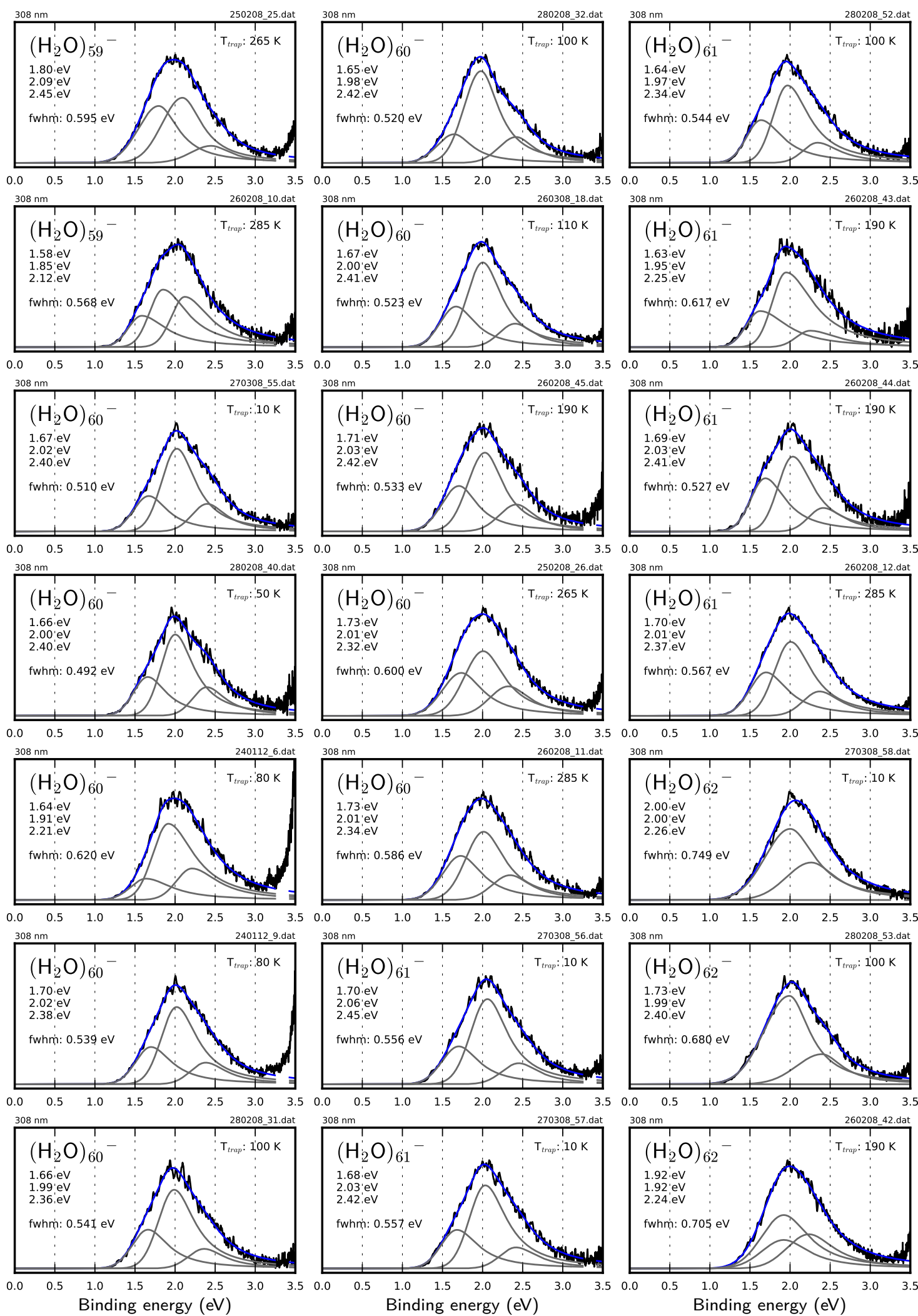
A.3. Water Cluster Anions (308 nm)
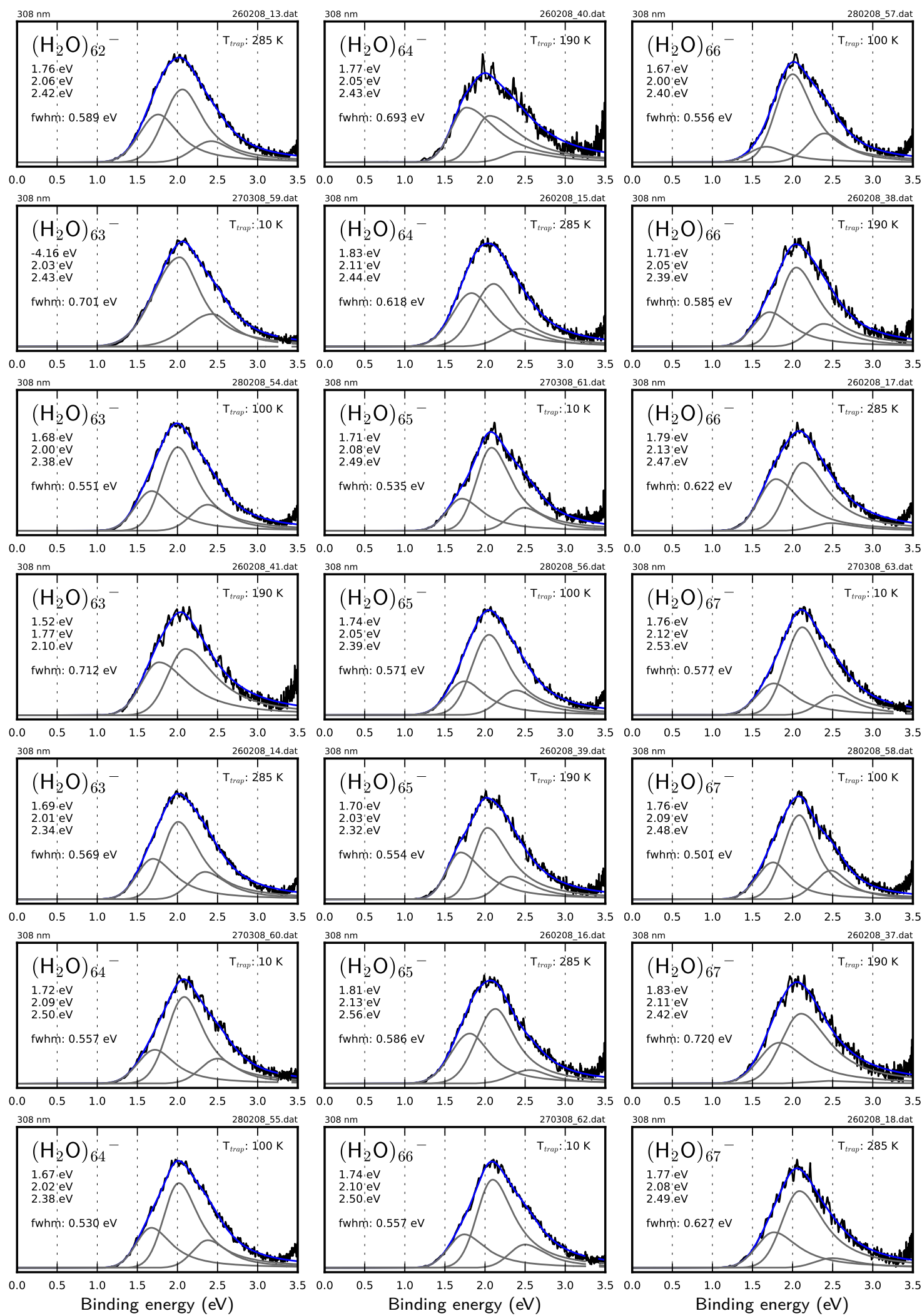

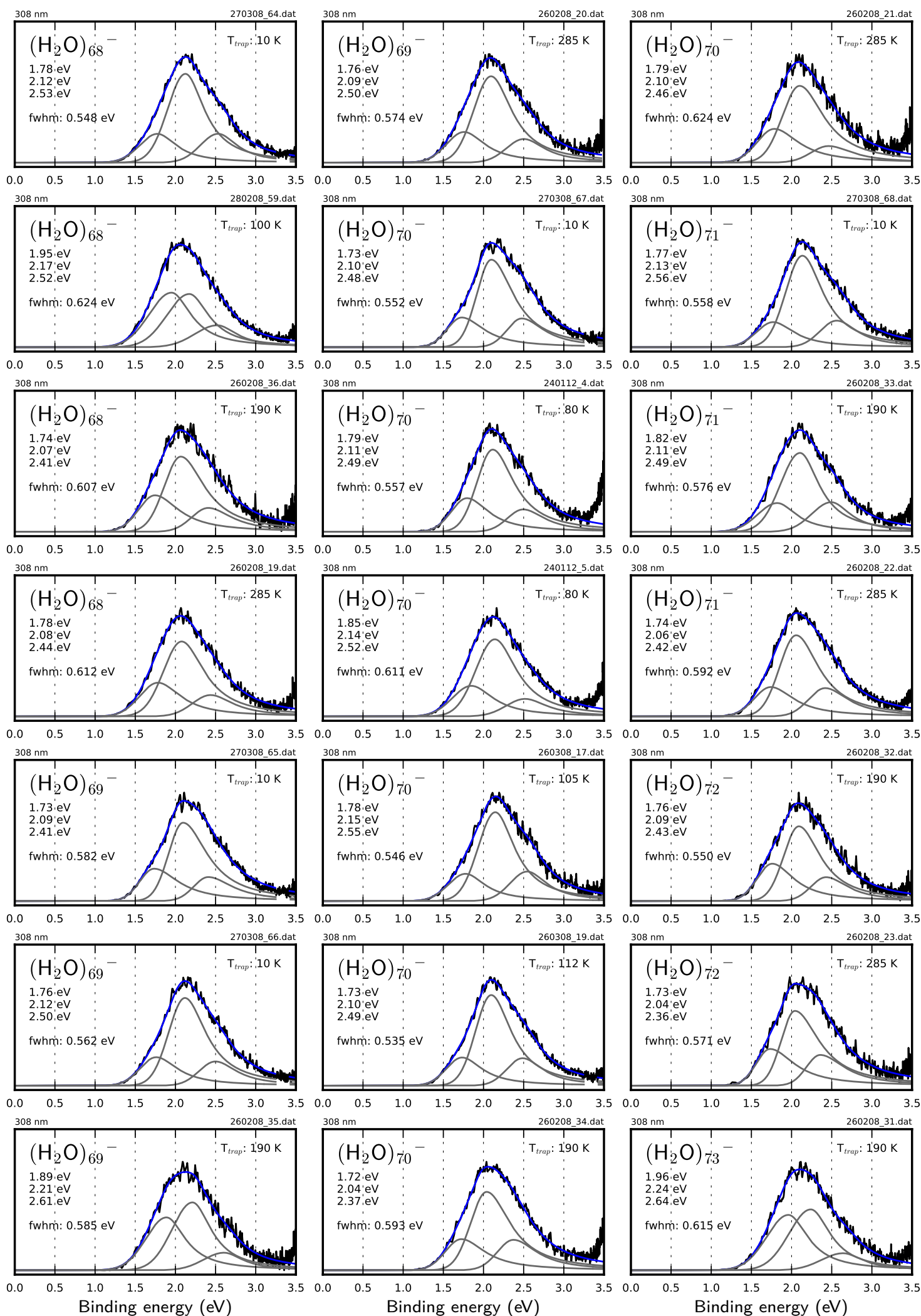

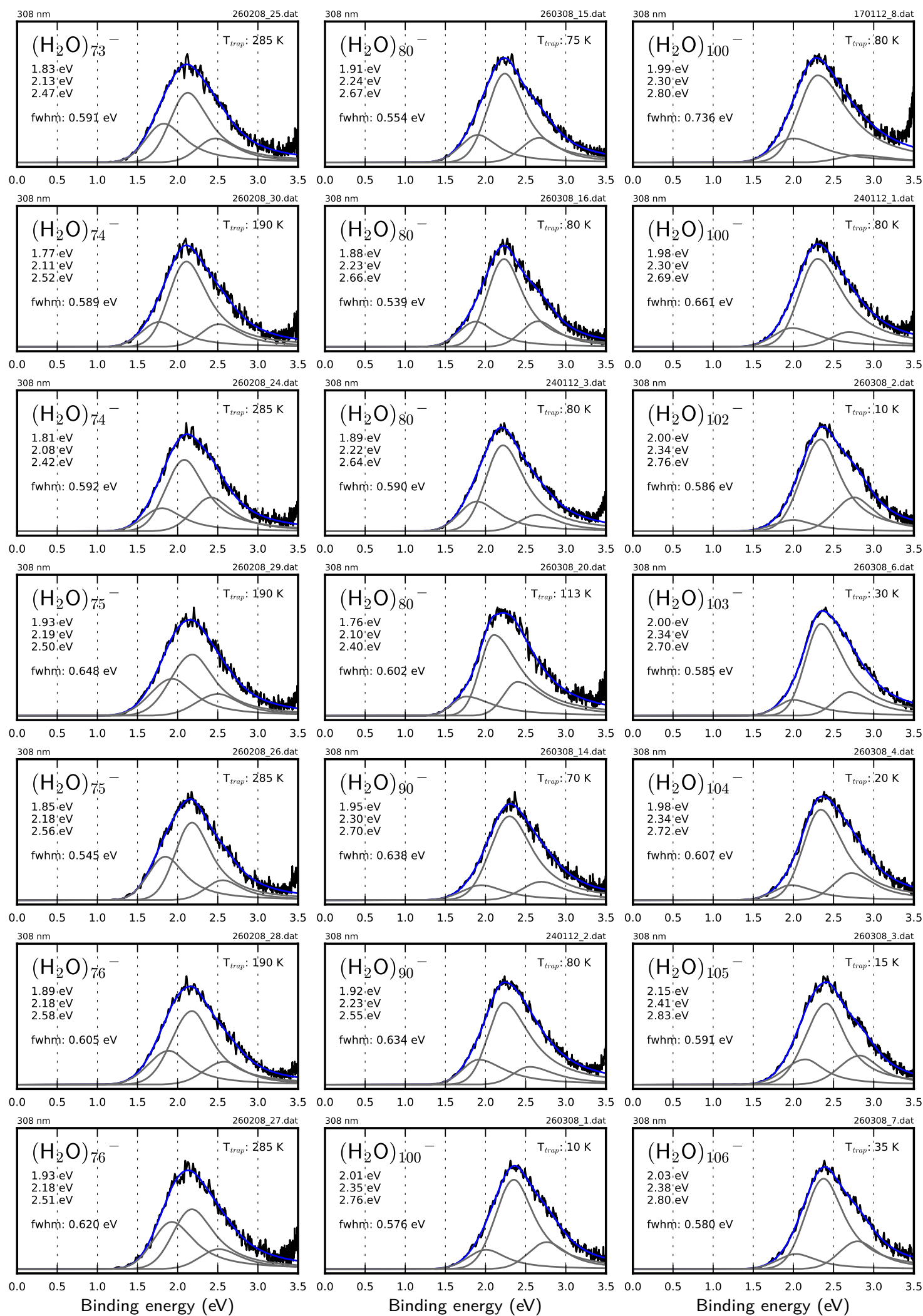

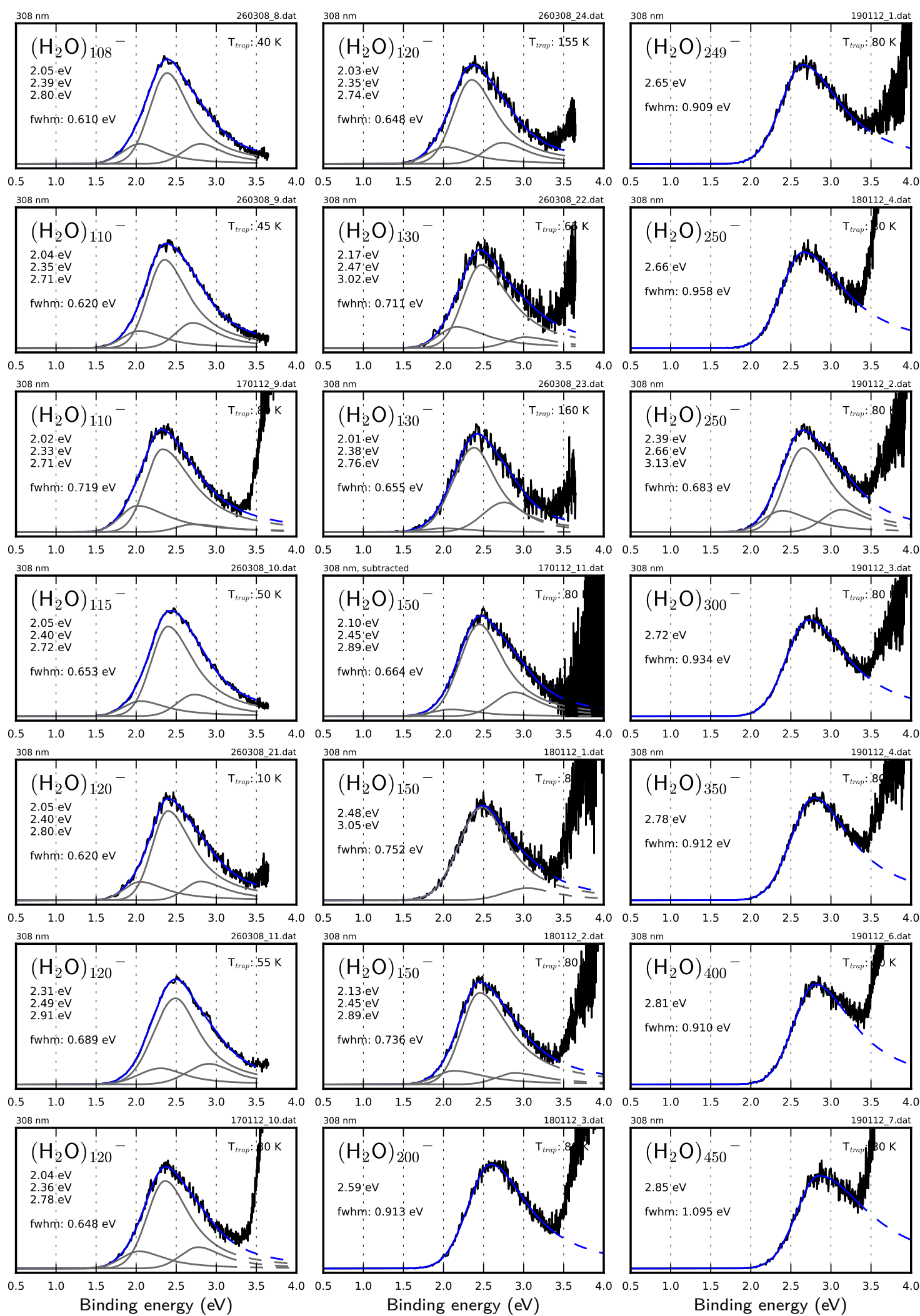

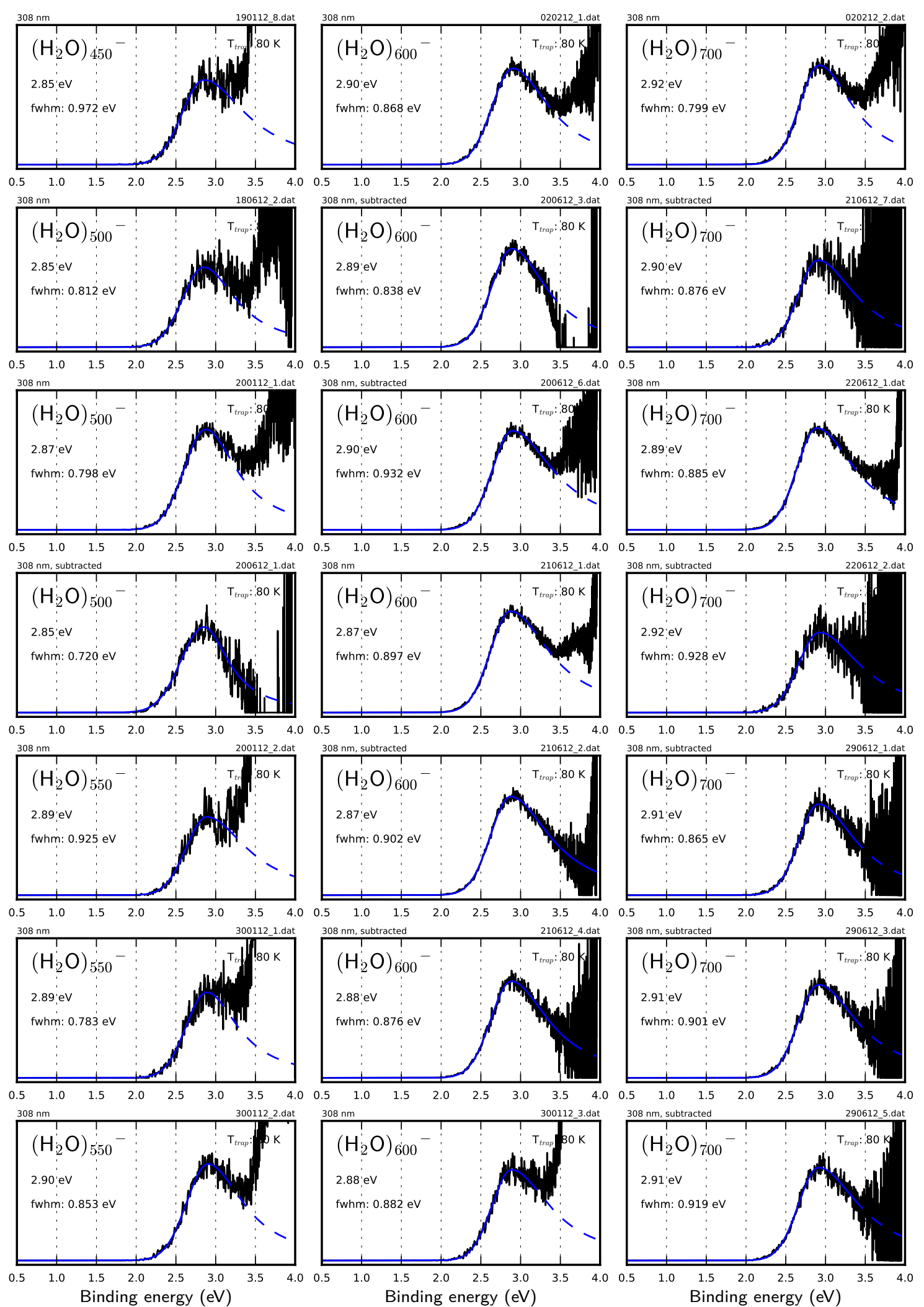

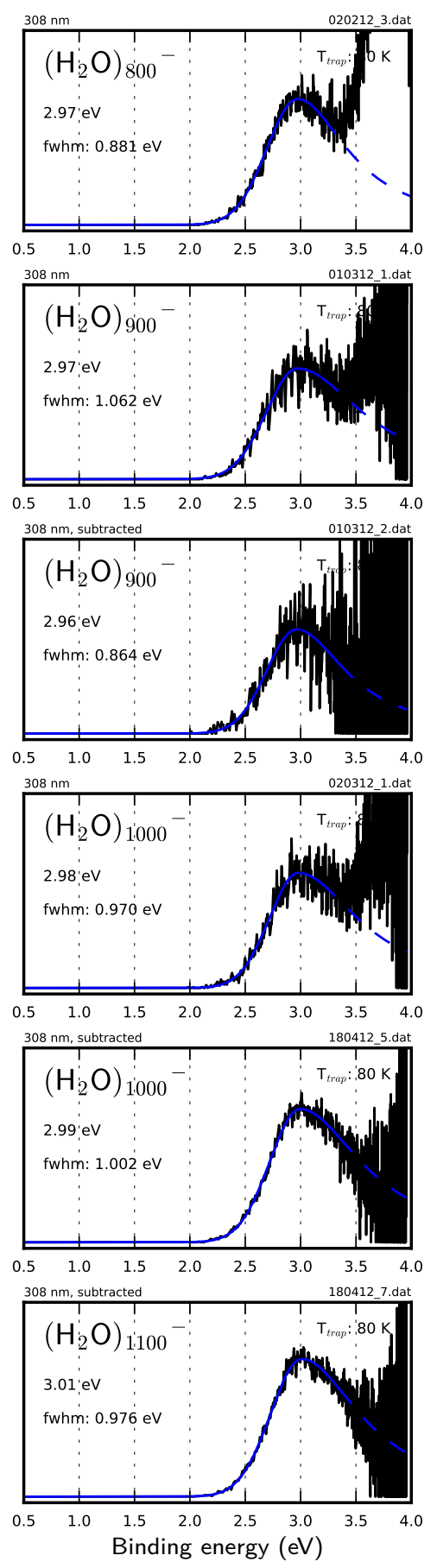


\section{A.4. Deuterated Water Cluster Anions (308 nm)}

Photoelectron spectra of heavy $\left(\mathrm{D}_{2} \mathrm{O}\right)_{\mathrm{n}}{ }^{-}$recorded at a detachment laser wavelength of $308 \mathrm{~nm}(4.02 \mathrm{eV})$. The intensity is given in arbitrary units. The fits show the results of the multi GL analysis according to the "best compromise" approach.
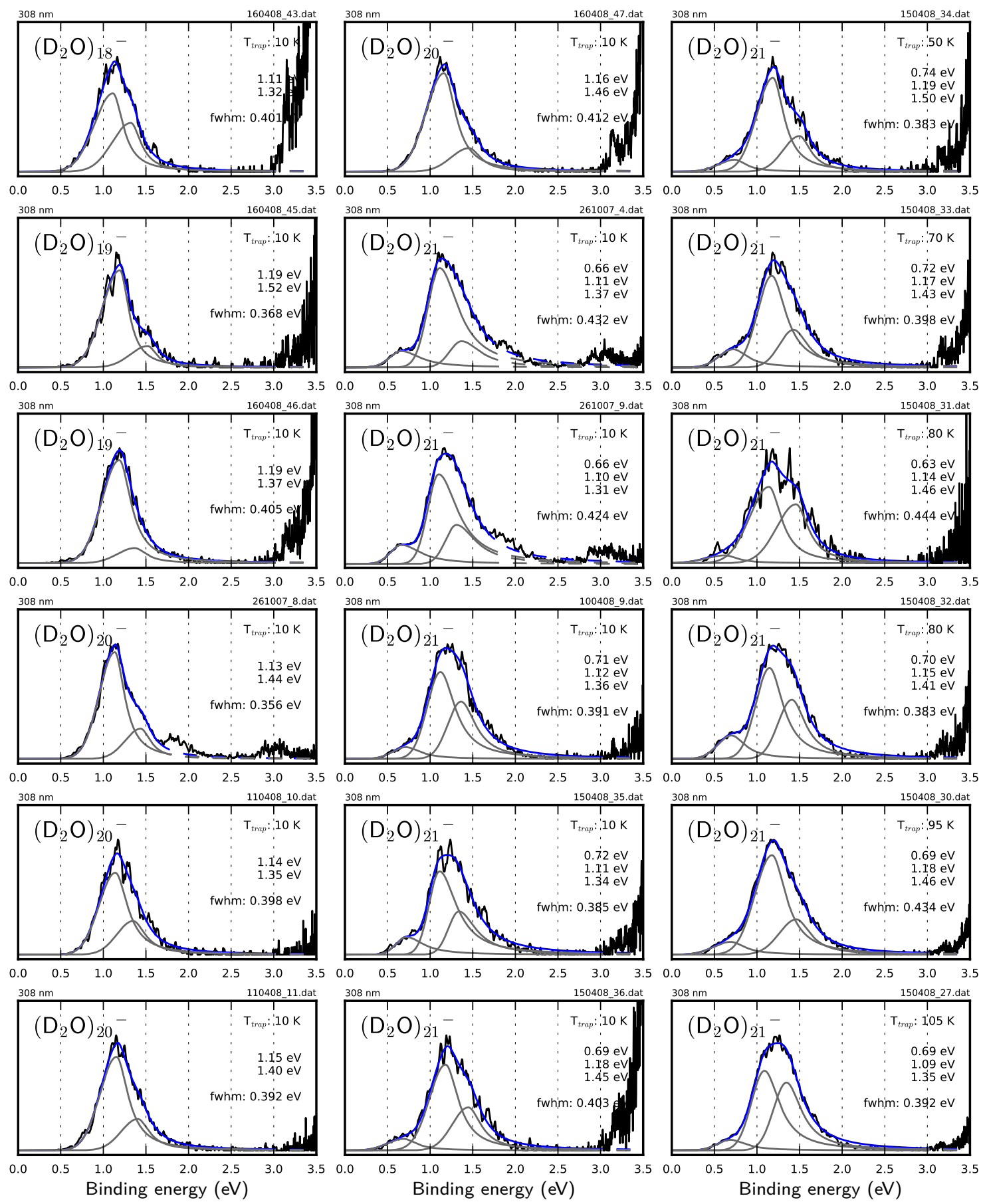

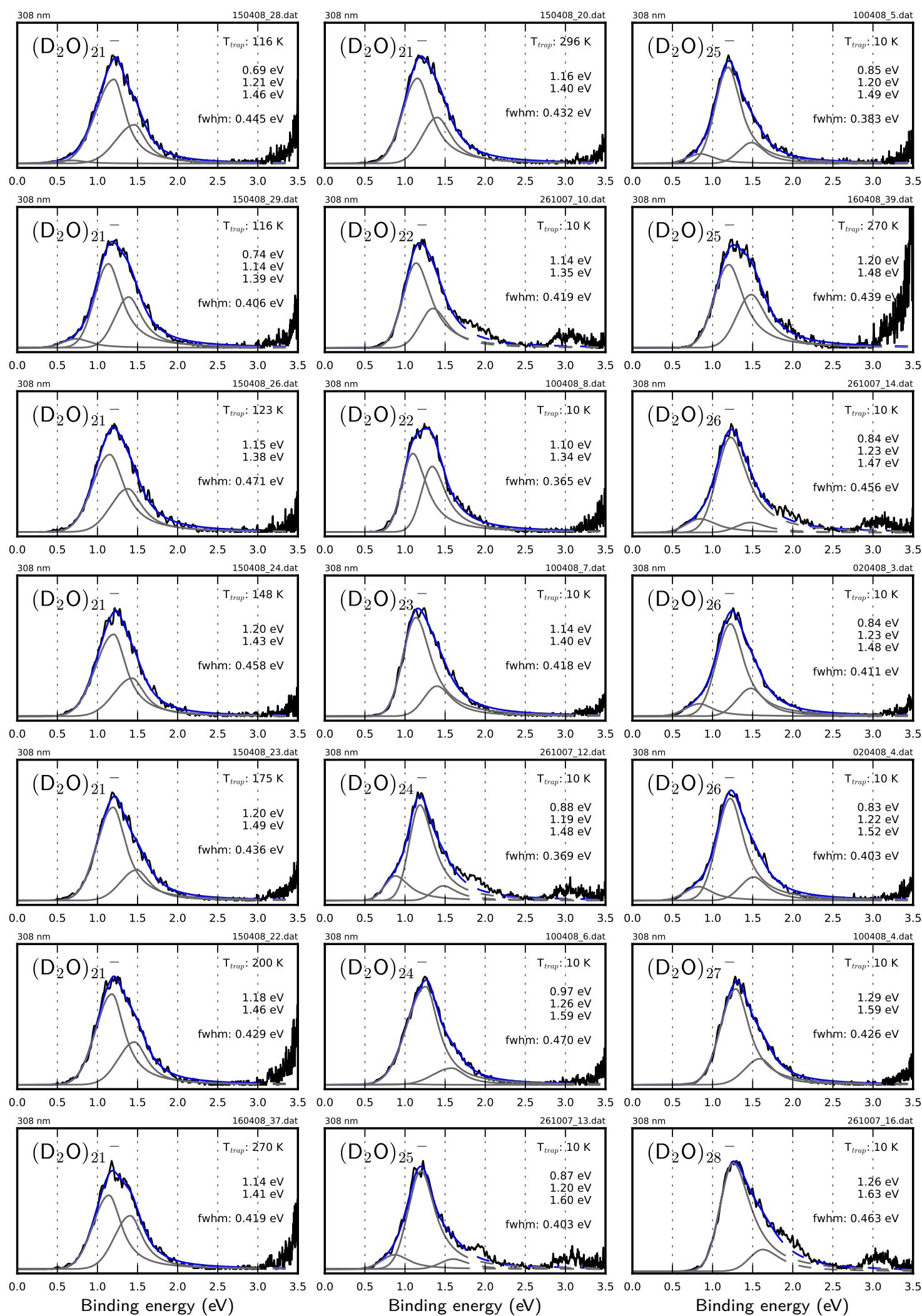
A.4. Deuterated Water Cluster Anions (308 nm)
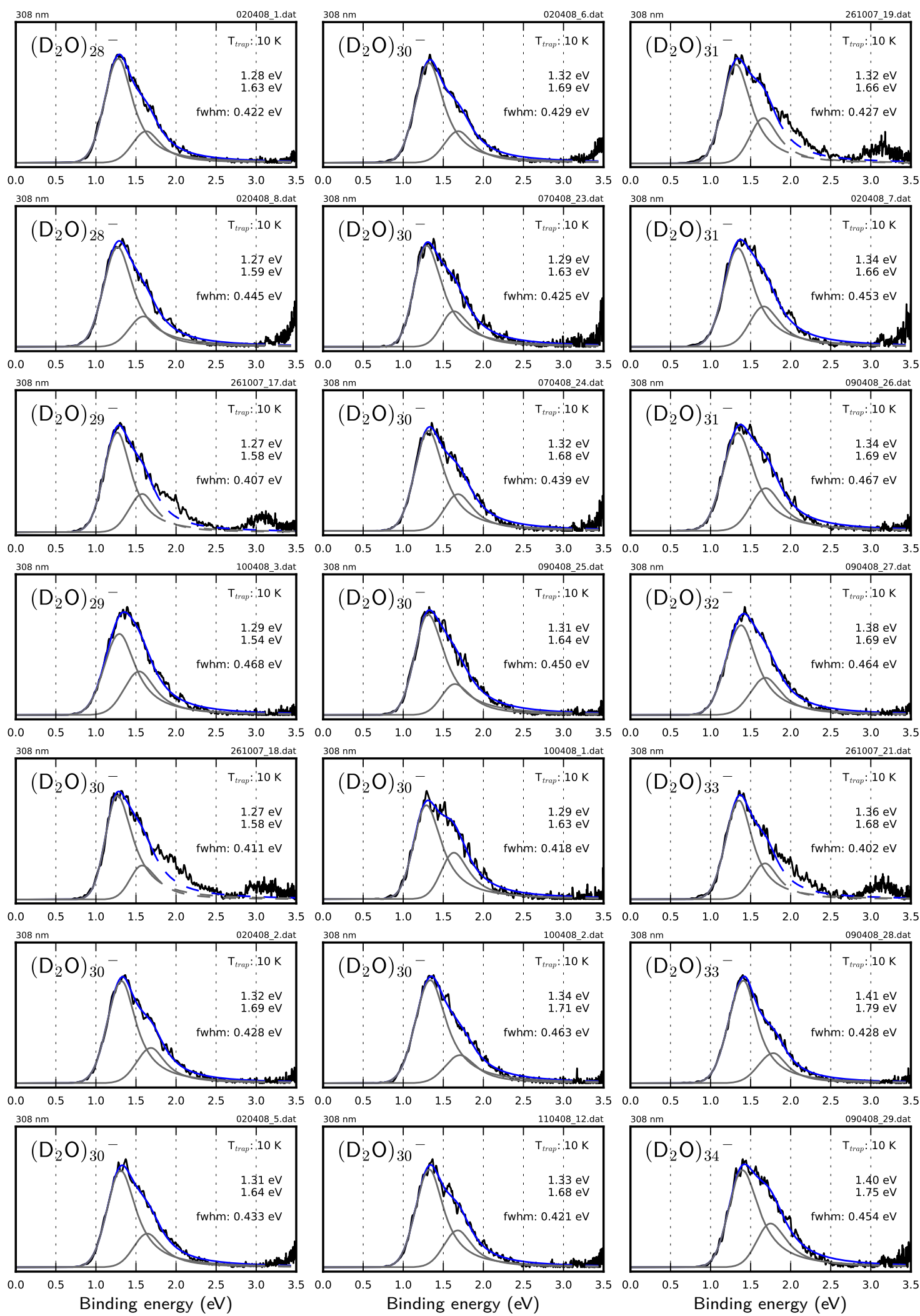

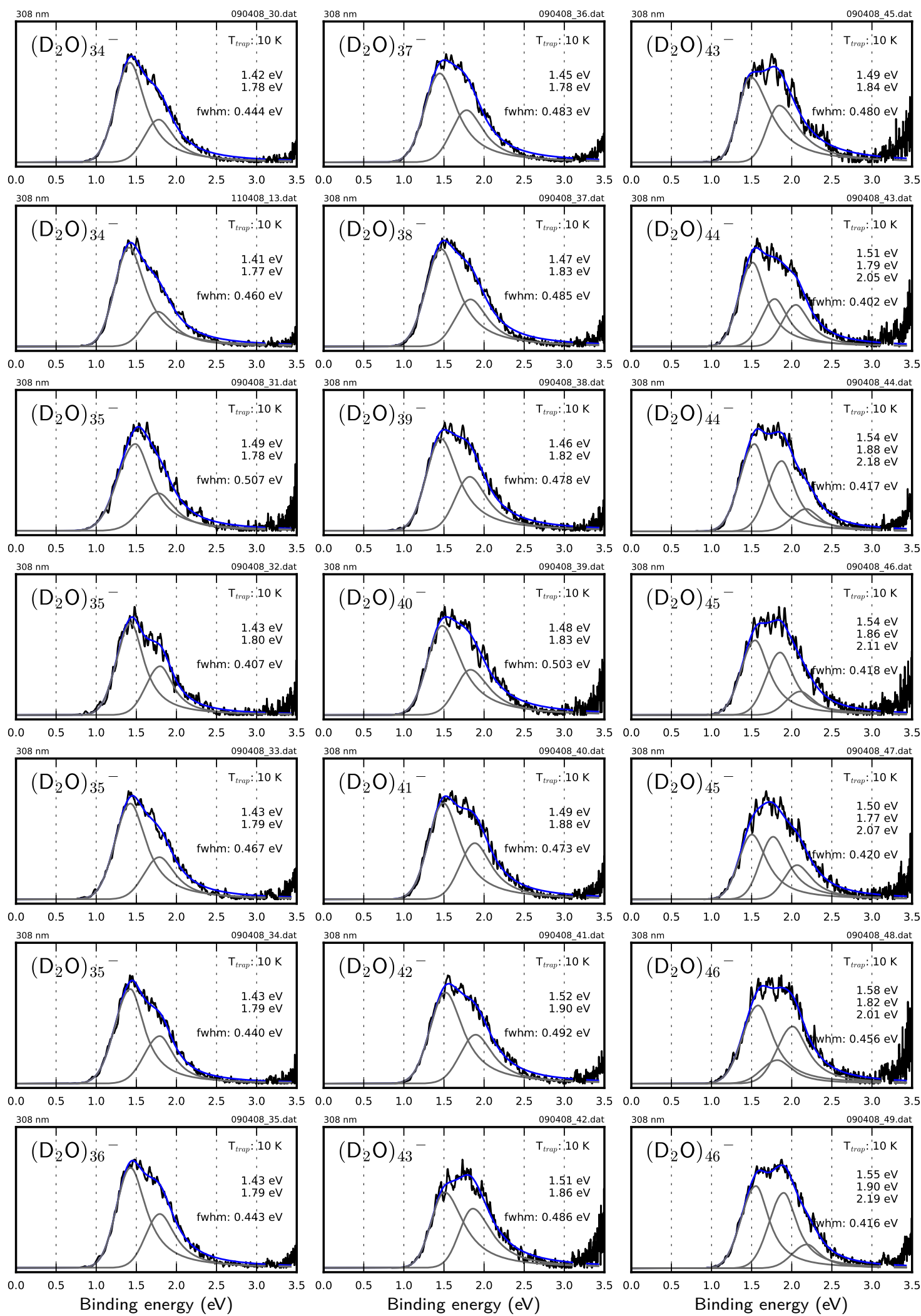
A.4. Deuterated Water Cluster Anions (308 nm)
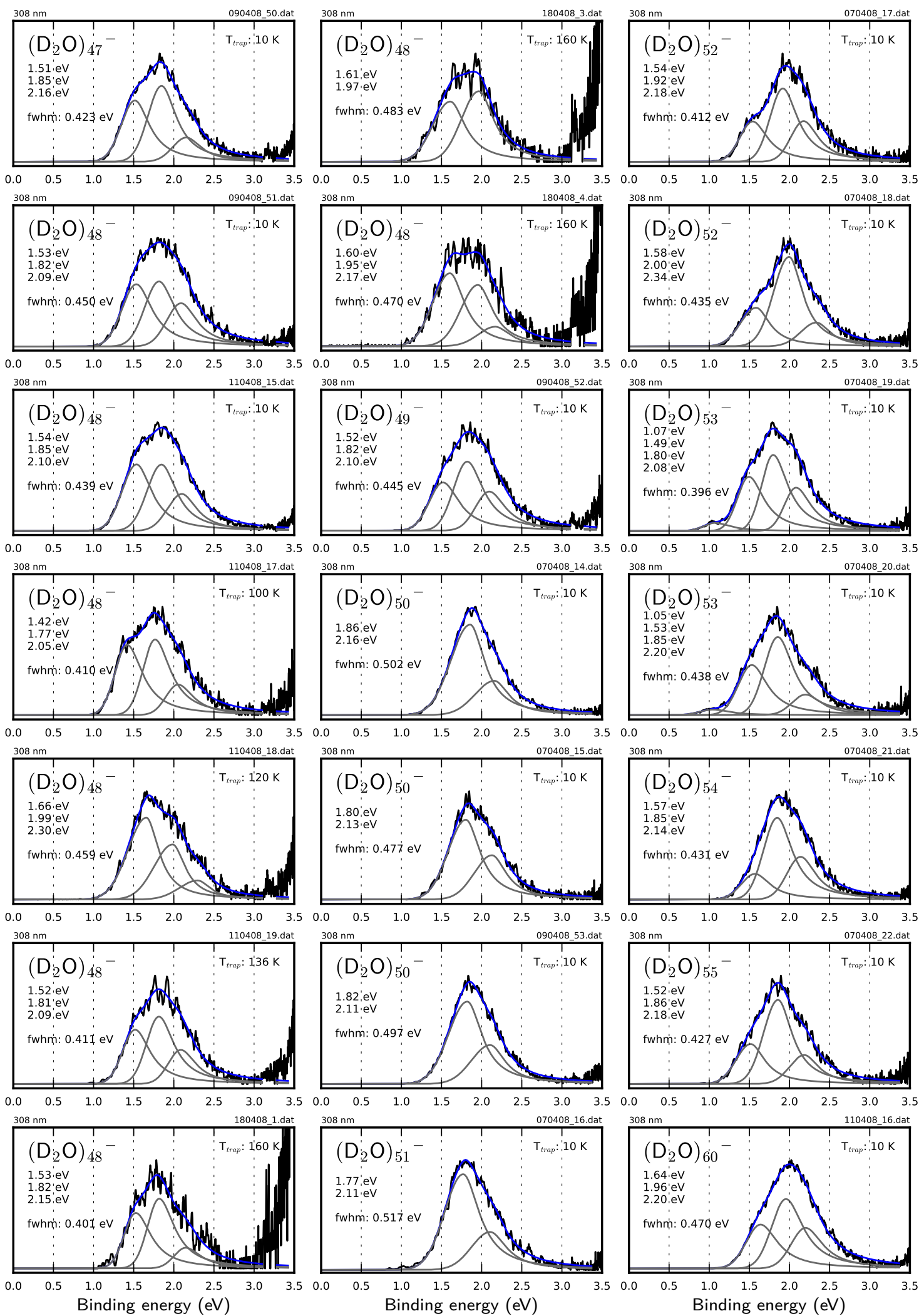
A. Photoelectron Spectra 


\section{B. Temperature Dependency}

\section{Water Cluster Anions}
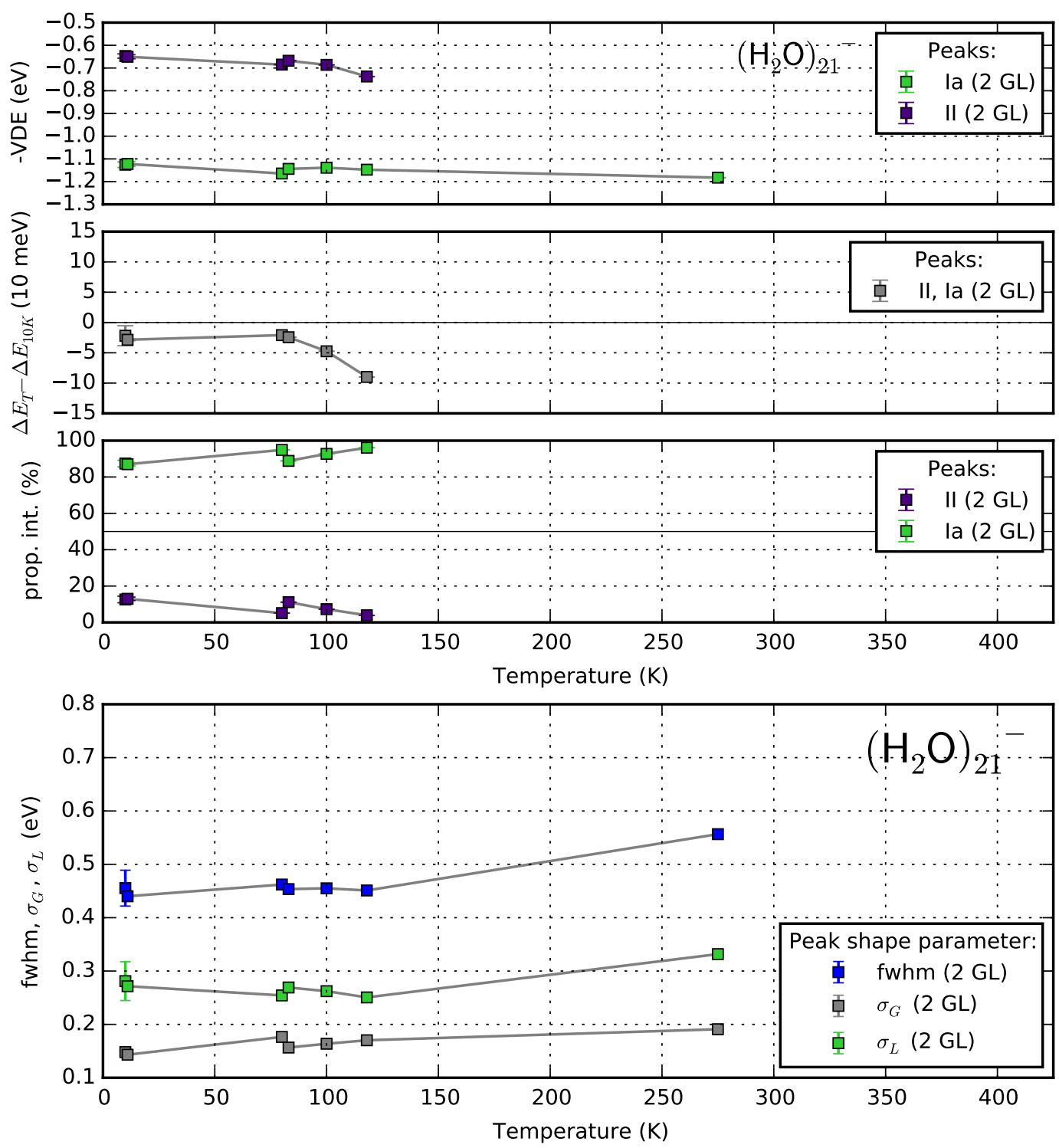

Figure B.1.: Temperature dependent evolution of the VDEs and peak parameters. 

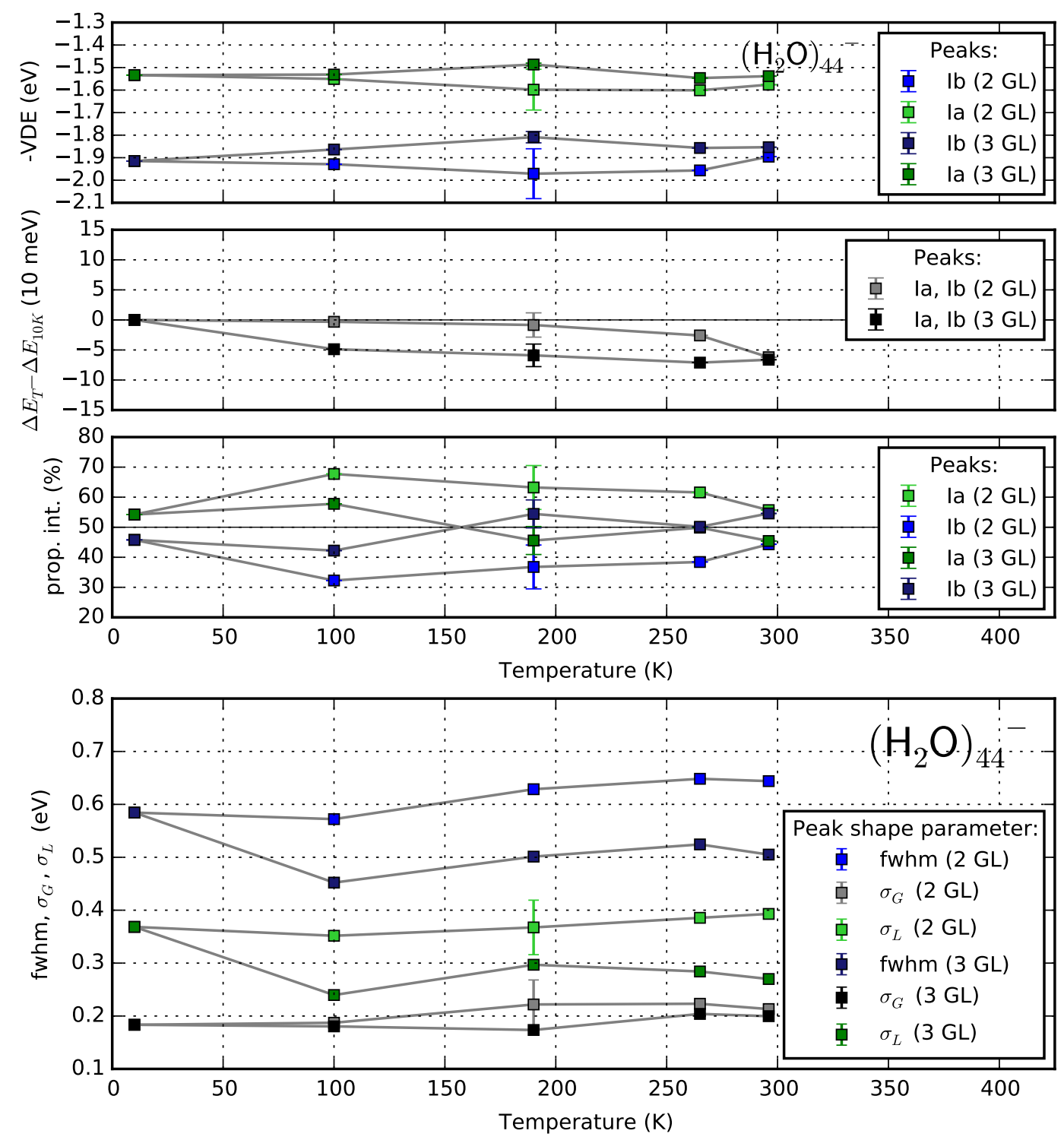

Figure B.2.: Temperature dependent evolution of the VDEs (upper three plots) and peak parameters (lower plot) of $\left(\mathrm{H}_{2} \mathrm{O}\right)_{44^{-}}$. 

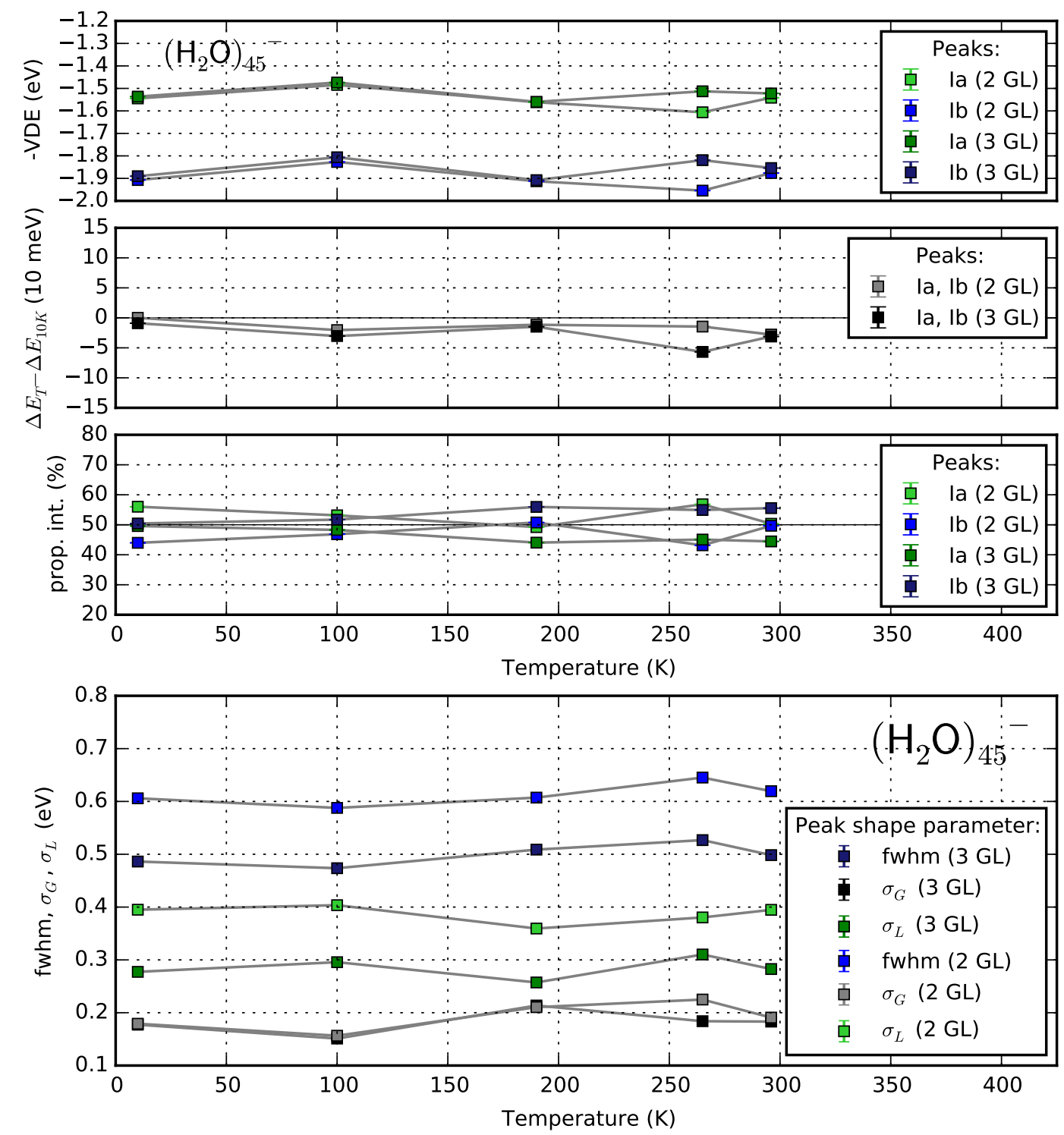

Figure B.3.: Temperature dependent evolution of the VDEs (upper three plots) and peak parameters (lower plot) of $\left(\mathrm{H}_{2} \mathrm{O}\right)_{45}{ }^{-}$. 

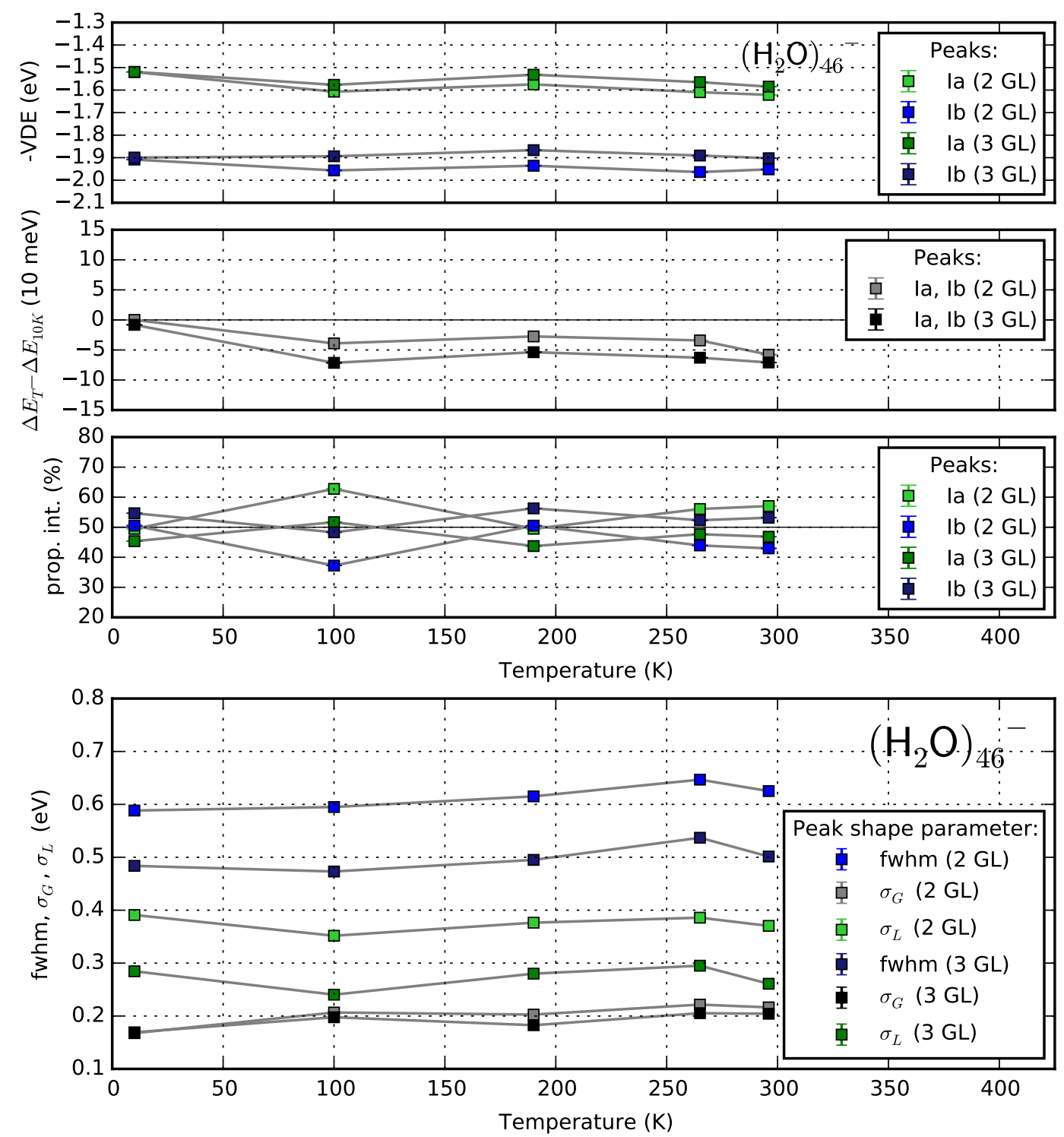

Figure B.4.: Temperature dependent evolution of the VDEs (upper three plots) and peak parameters (lower plot) of $\left(\mathrm{H}_{2} \mathrm{O}\right)_{46}{ }^{-}$. 

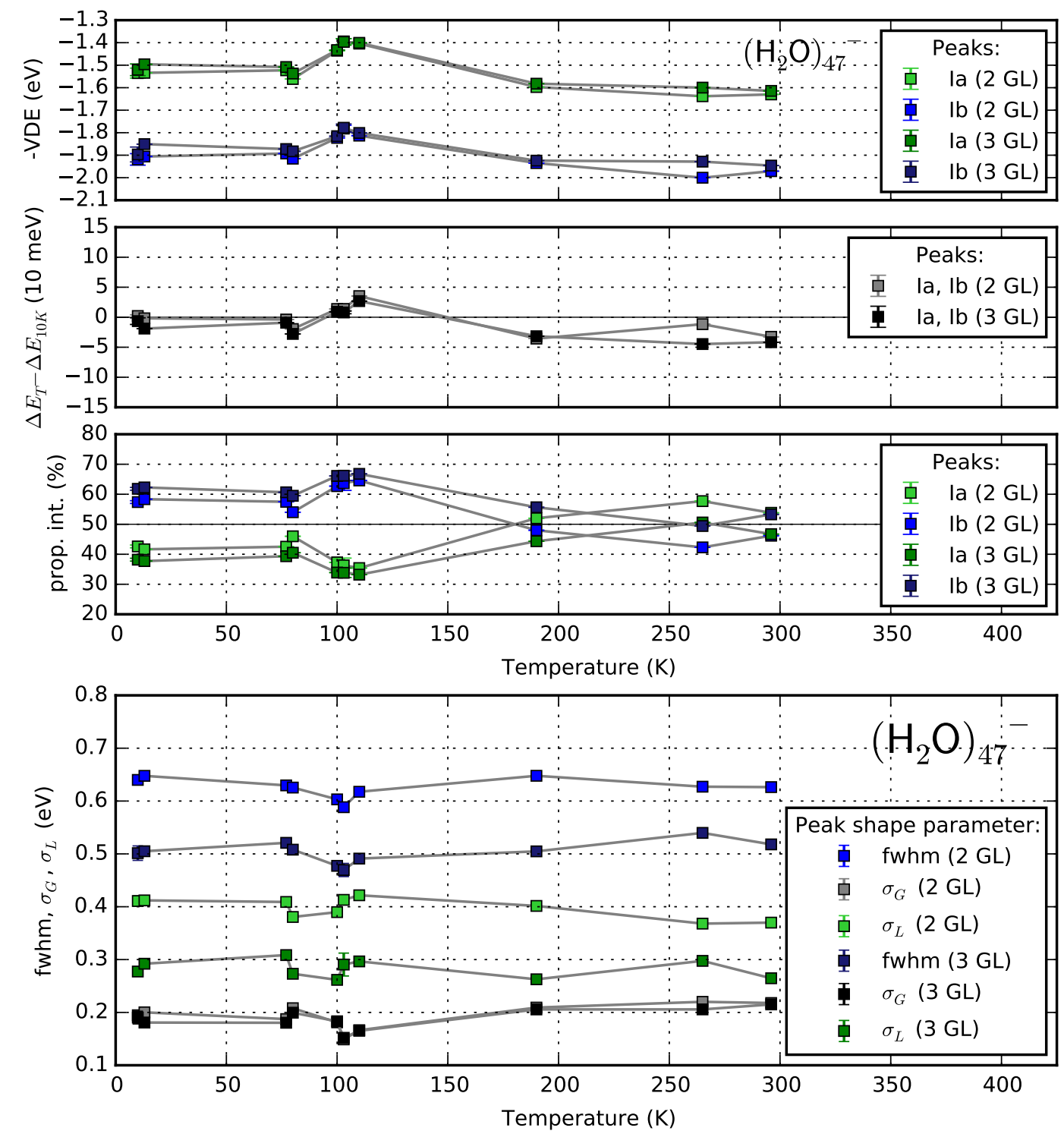

Figure B.5.: Temperature dependent evolution of the VDEs (upper three plots) and peak parameters (lower plot) of $\left(\mathrm{H}_{2} \mathrm{O}\right)_{47}{ }^{-}$. 

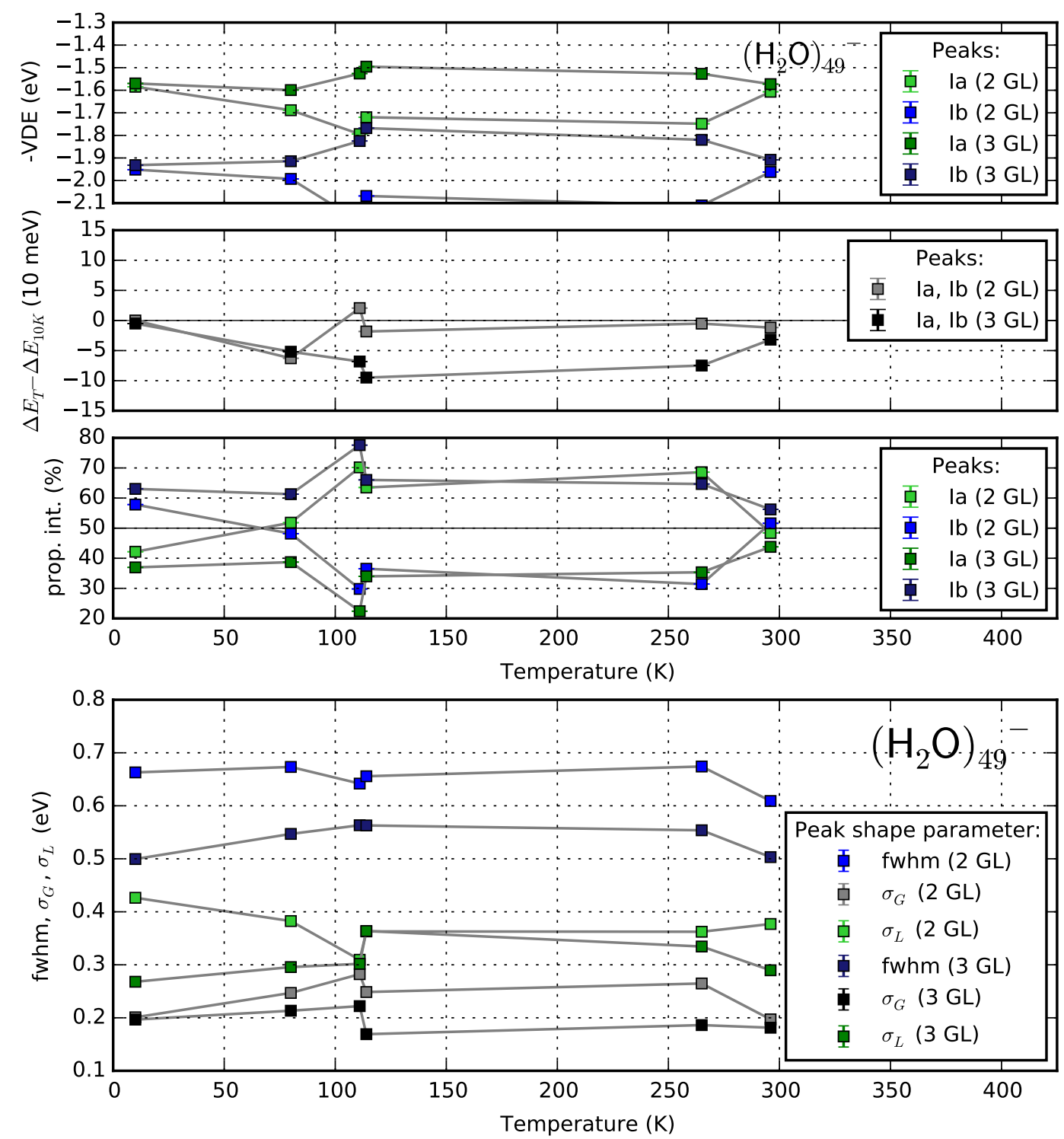

Figure B.6.: Temperature dependent evolution of the VDEs (upper three plots) and peak parameters (lower plot) of $\left(\mathrm{H}_{2} \mathrm{O}\right)_{49}{ }^{-}$. 

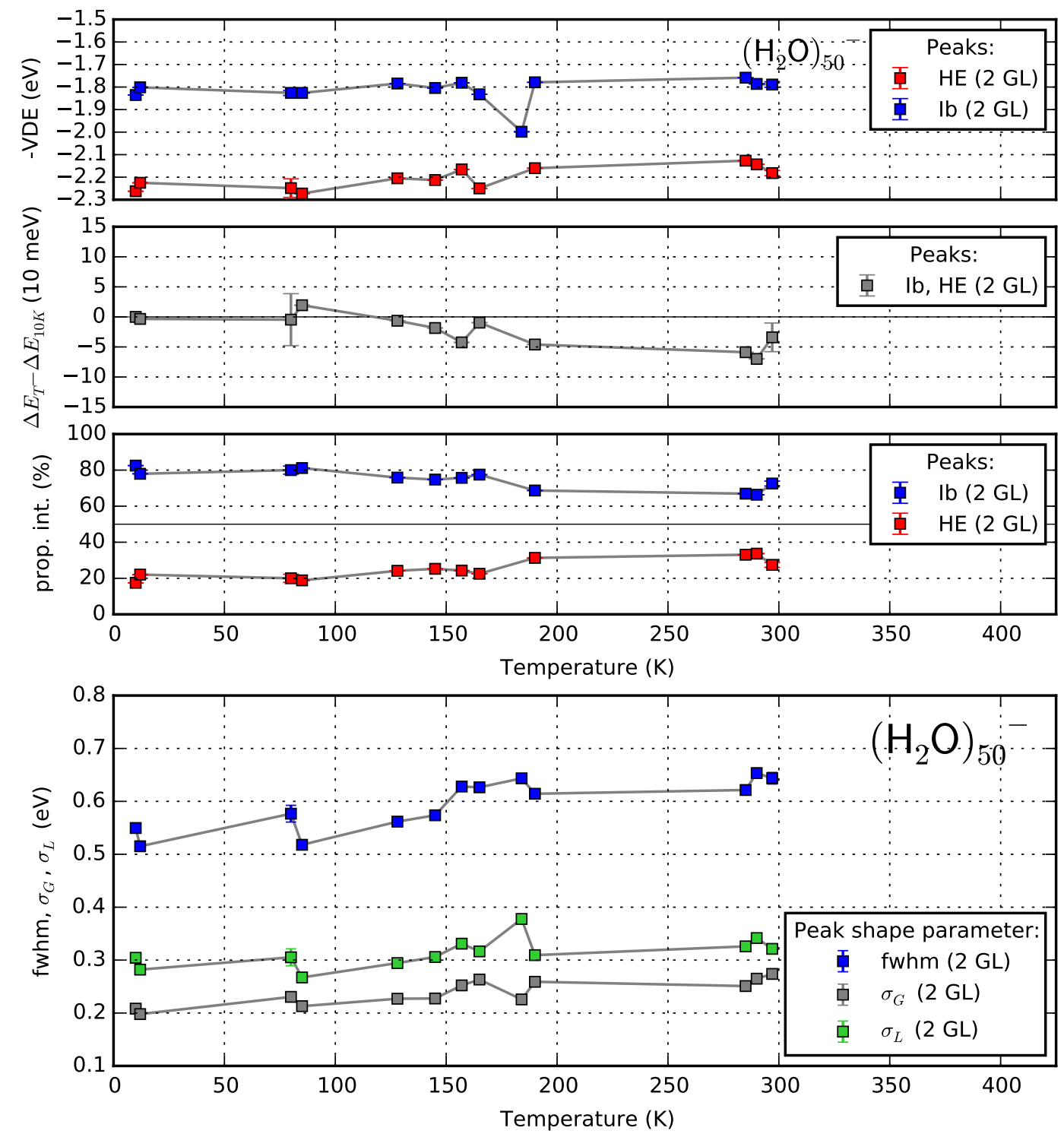

Figure B.7.: Temperature dependent evolution of the VDEs (upper three plots) and peak parameters (lower plot) of $\left(\mathrm{H}_{2} \mathrm{O}\right)_{50}{ }^{-}$. 

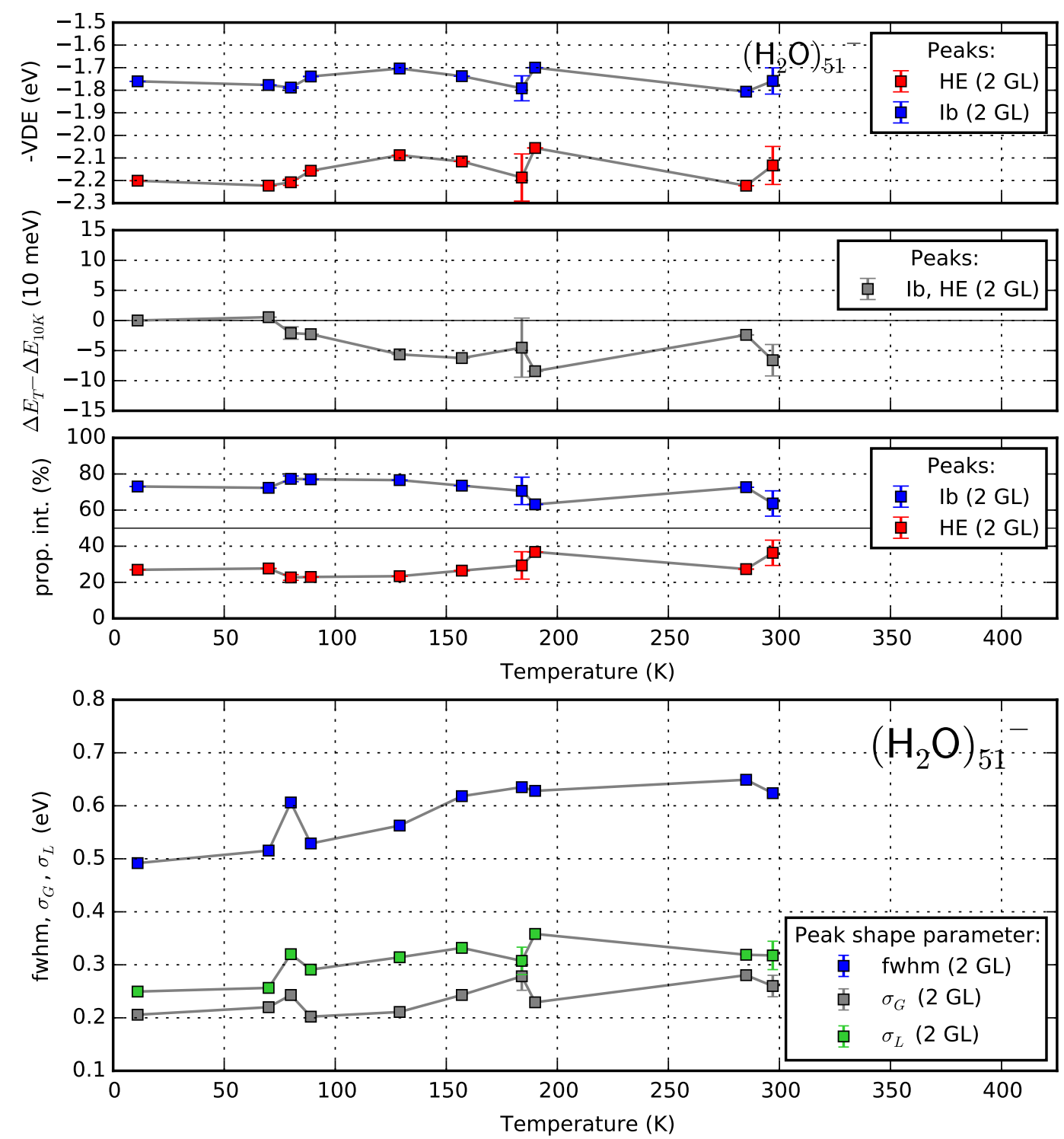

Figure B.8.: Temperature dependent evolution of the VDEs (upper three plots) and peak parameters (lower plot) of $\left(\mathrm{H}_{2} \mathrm{O}\right)_{51}{ }^{-}$. 

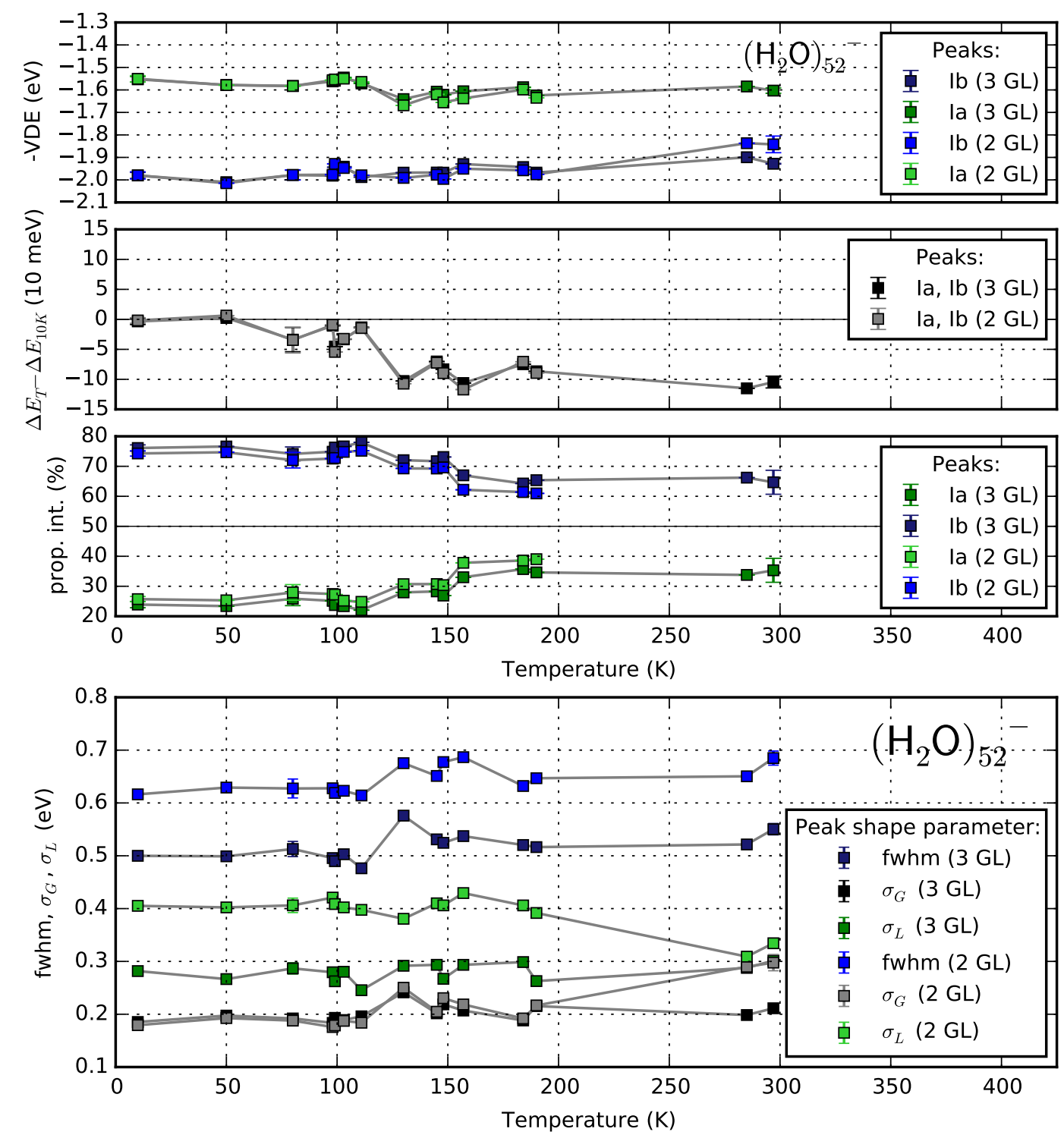

Figure B.9.: Temperature dependent evolution of the VDEs (upper three plots) and peak parameters (lower plot) of $\left(\mathrm{H}_{2} \mathrm{O}\right)_{52}{ }^{-}$. 

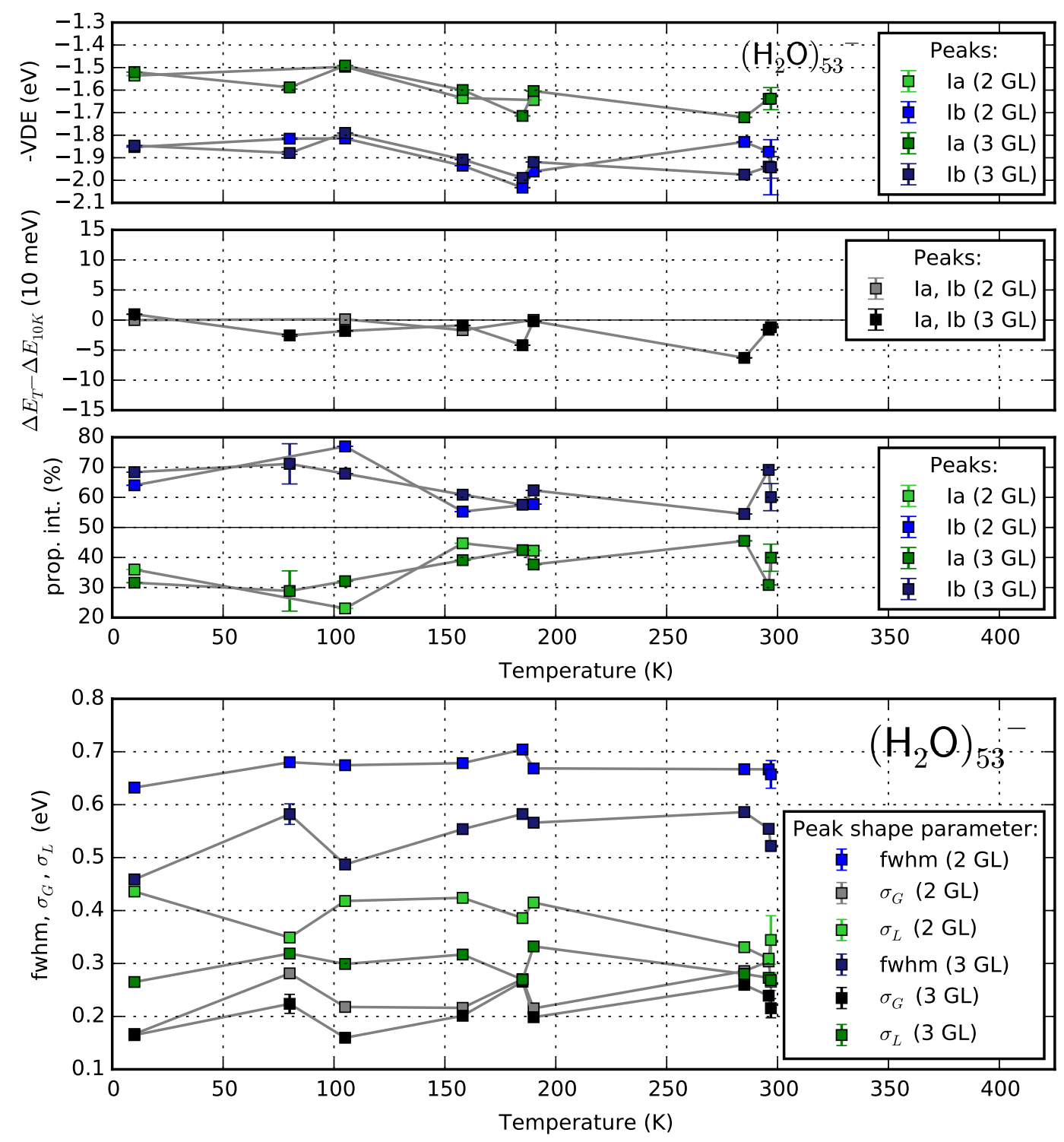

Figure B.10.: Temperature dependent evolution of the VDEs (upper three plots) and peak parameters (lower plot) of $\left(\mathrm{H}_{2} \mathrm{O}\right)_{53}{ }^{-}$. 

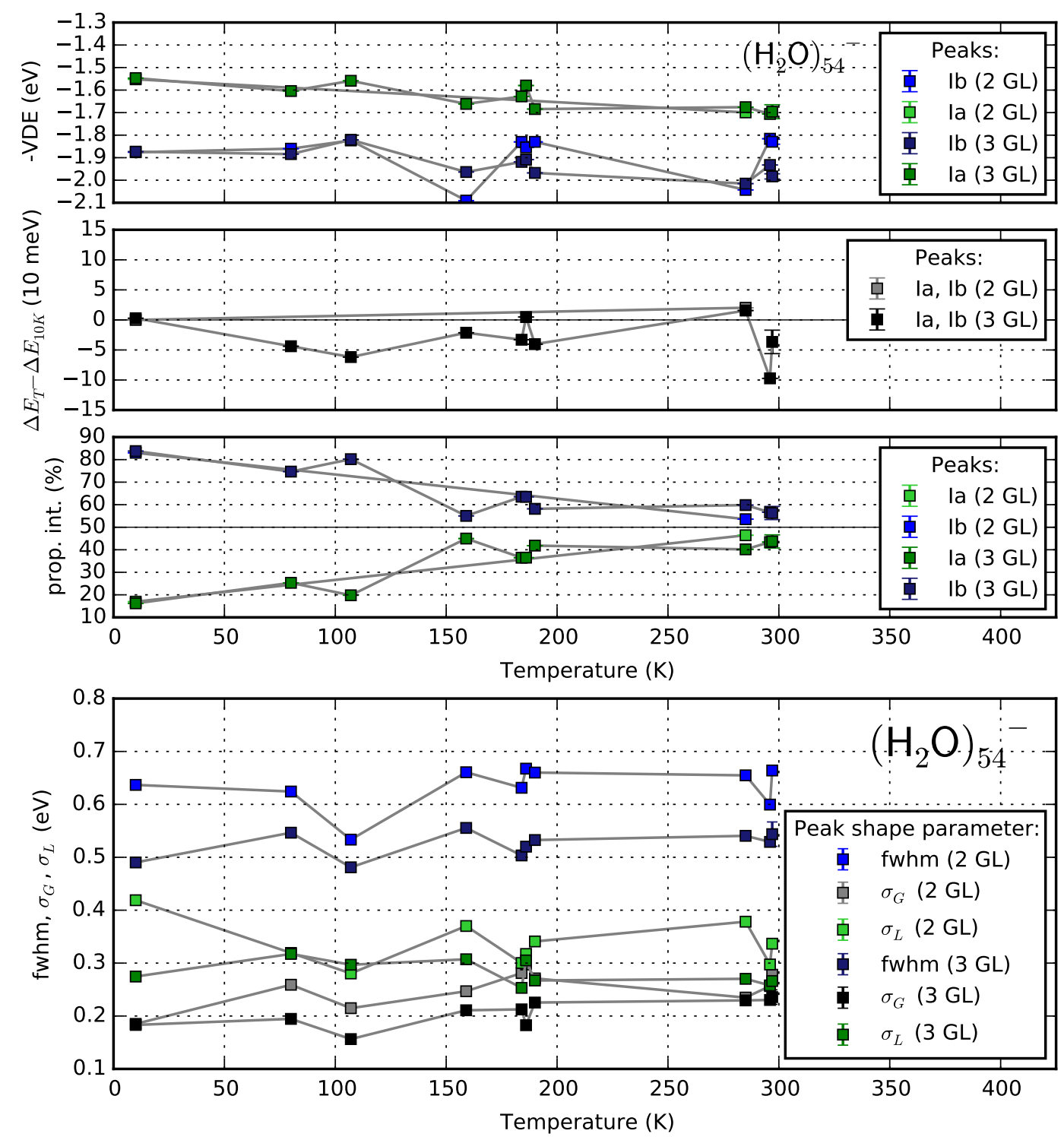

Figure B.11.: Temperature dependent evolution of the VDEs (upper three plots) and peak parameters (lower plot) of $\left(\mathrm{H}_{2} \mathrm{O}\right)_{54}{ }^{-}$. 

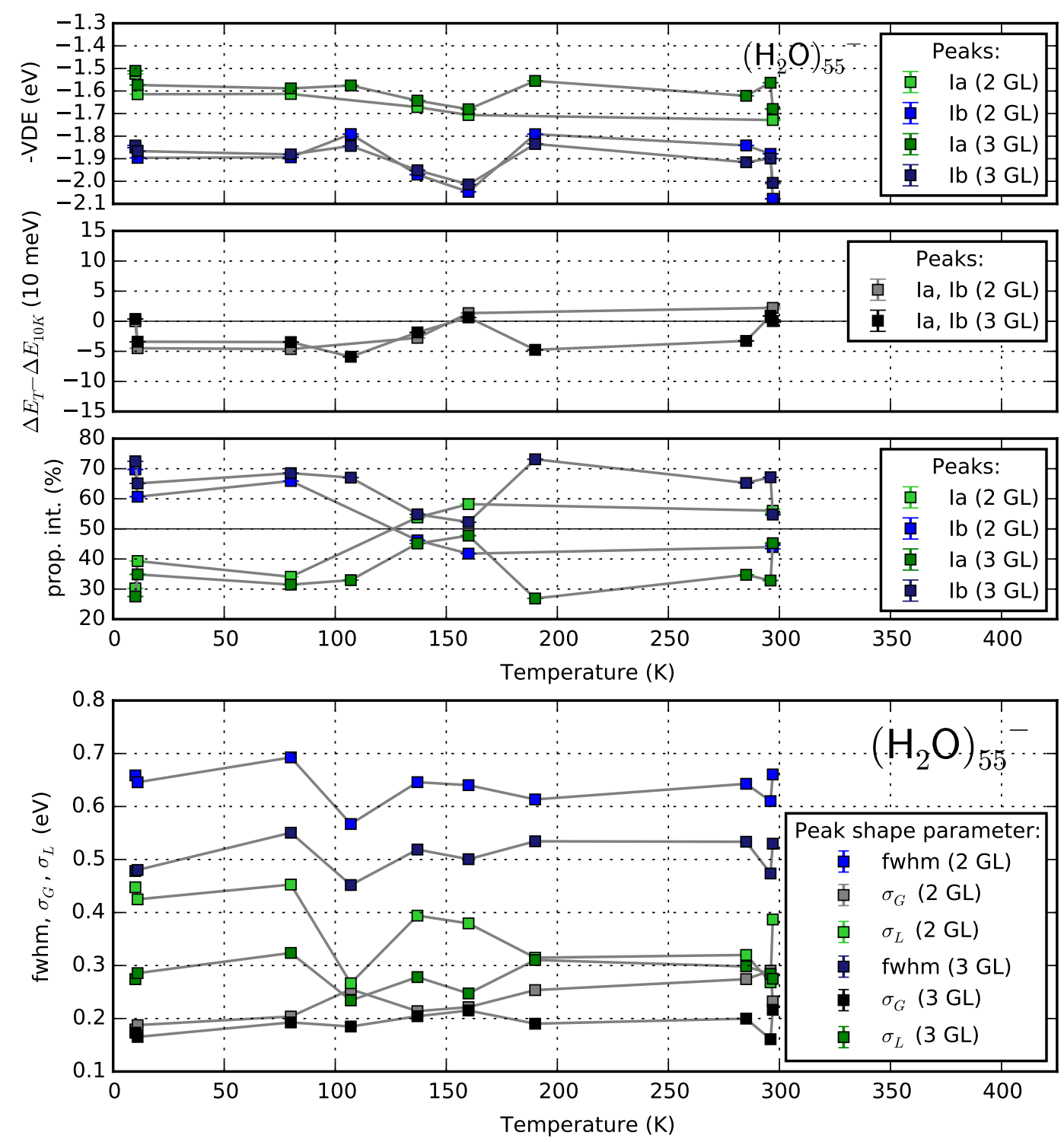

Figure B.12.: Temperature dependent evolution of the VDEs (upper three plots) and peak parameters (lower plot) of $\left(\mathrm{H}_{2} \mathrm{O}\right)_{55}{ }^{-}$. 

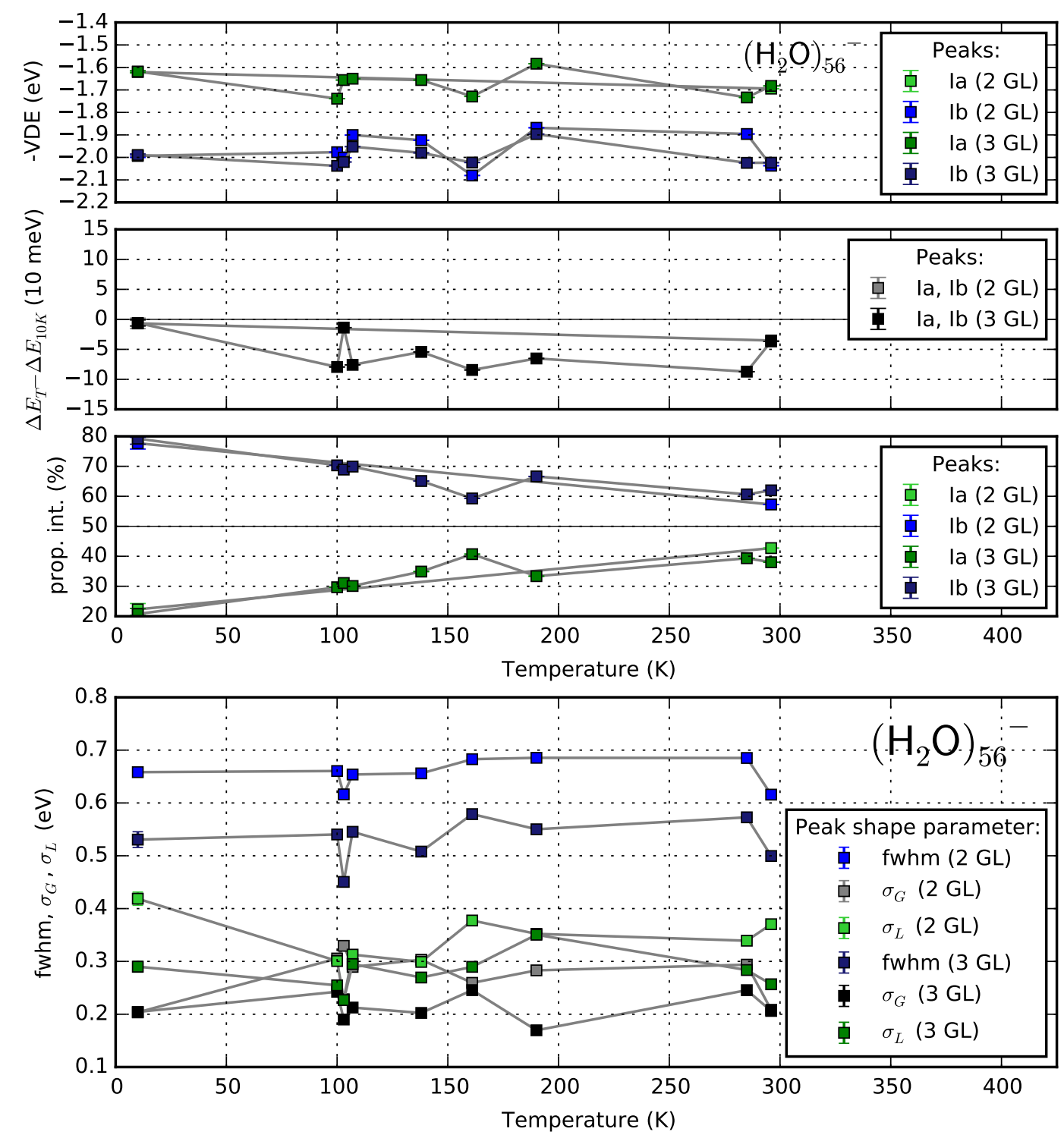

Figure B.13.: Temperature dependent evolution of the VDEs (upper three plots) and peak parameters (lower plot) of $\left(\mathrm{H}_{2} \mathrm{O}\right)_{56}{ }^{-}$. 

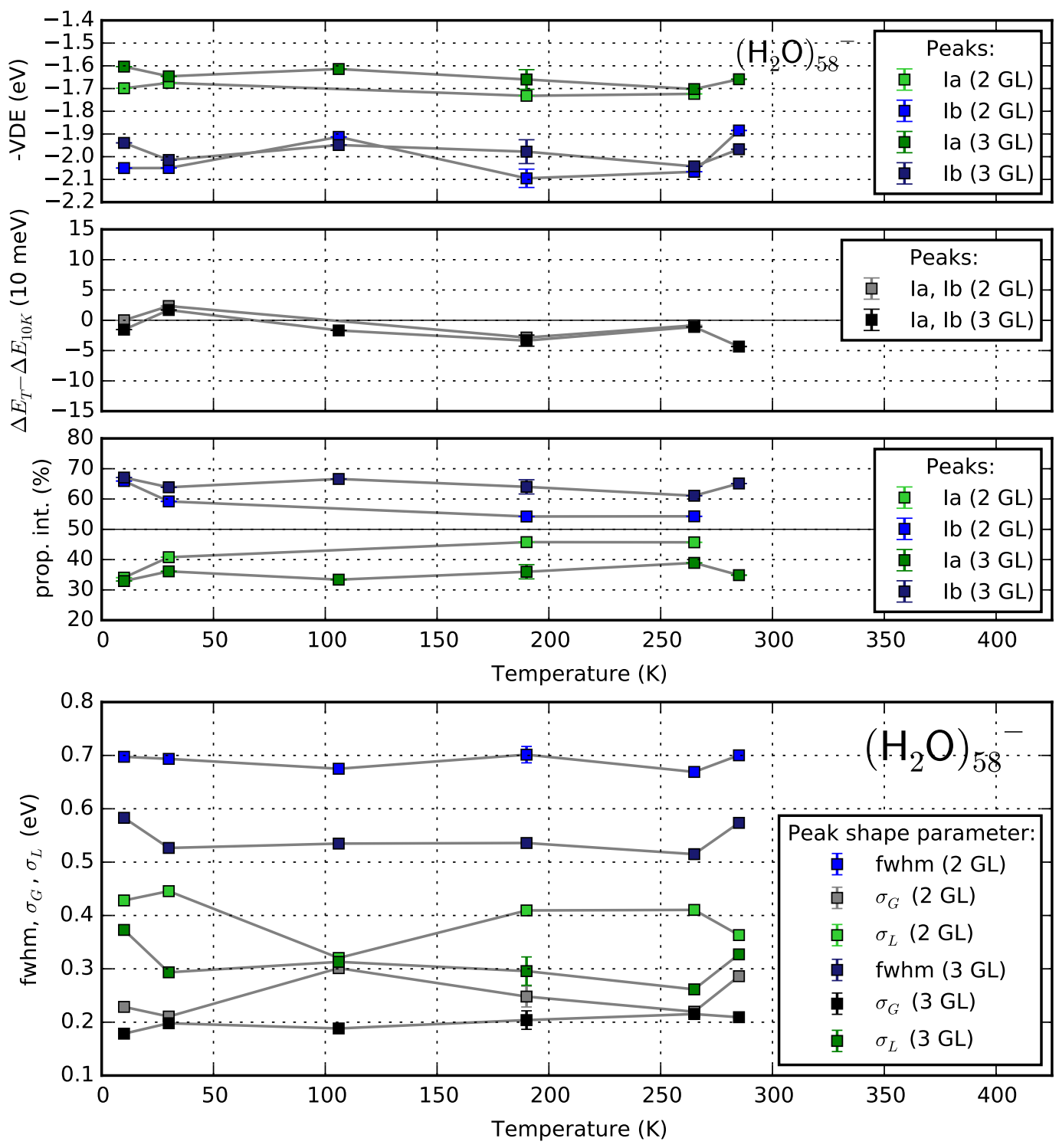

Figure B.14.: Temperature dependent evolution of the VDEs (upper three plots) and peak parameters (lower plot) of $\left(\mathrm{H}_{2} \mathrm{O}\right)_{58}{ }^{-}$. 

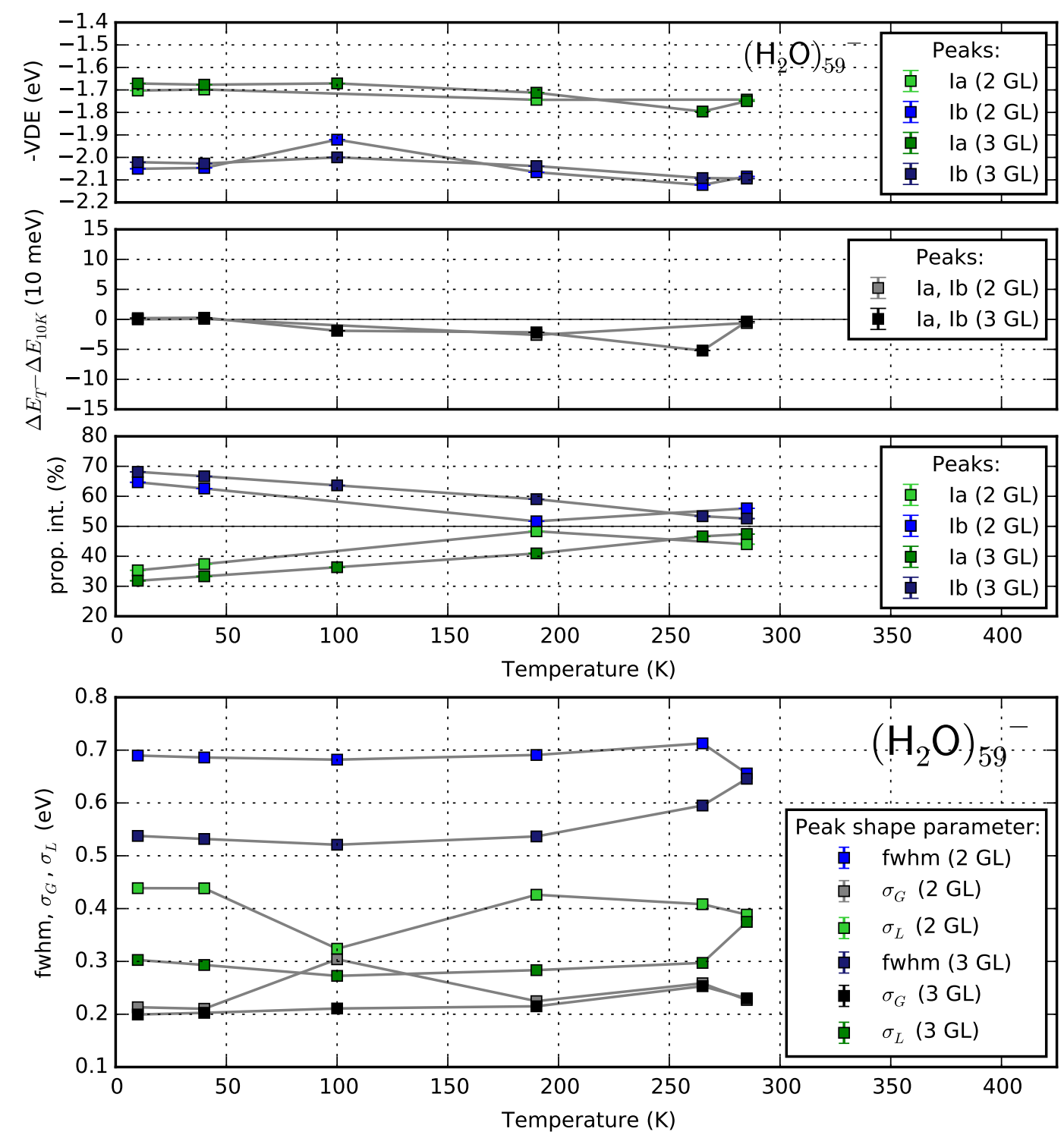

Figure B.15.: Temperature dependent evolution of the VDEs (upper three plots) and peak parameters (lower plot) of $\left(\mathrm{H}_{2} \mathrm{O}\right)_{59}{ }^{-}$. 

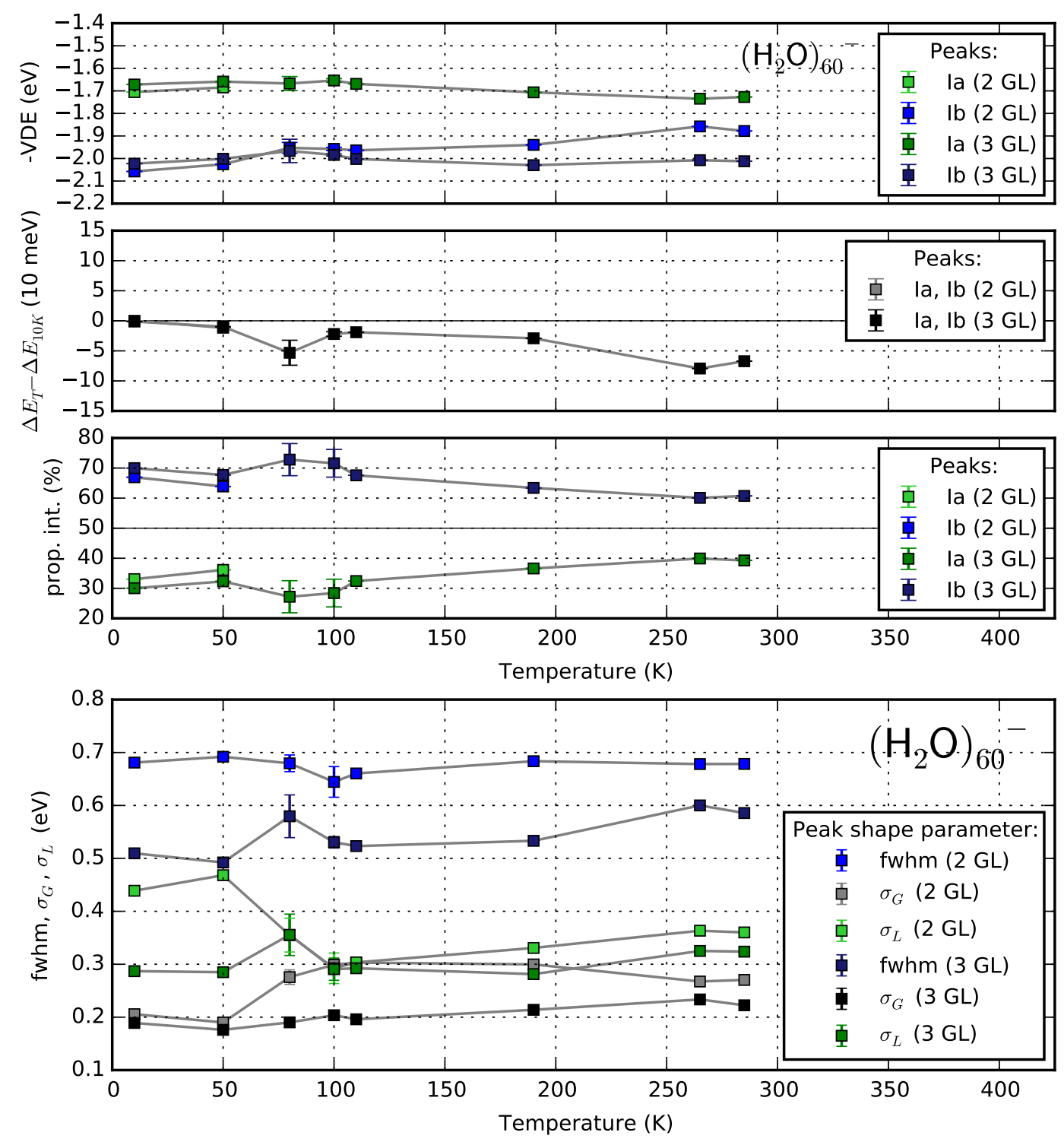

Figure B.16.: Temperature dependent evolution of the VDEs (upper three plots) and peak parameters (lower plot) of $\left(\mathrm{H}_{2} \mathrm{O}\right)_{60}{ }^{-}$. 


\section{Deuterated Water Cluster Anions}
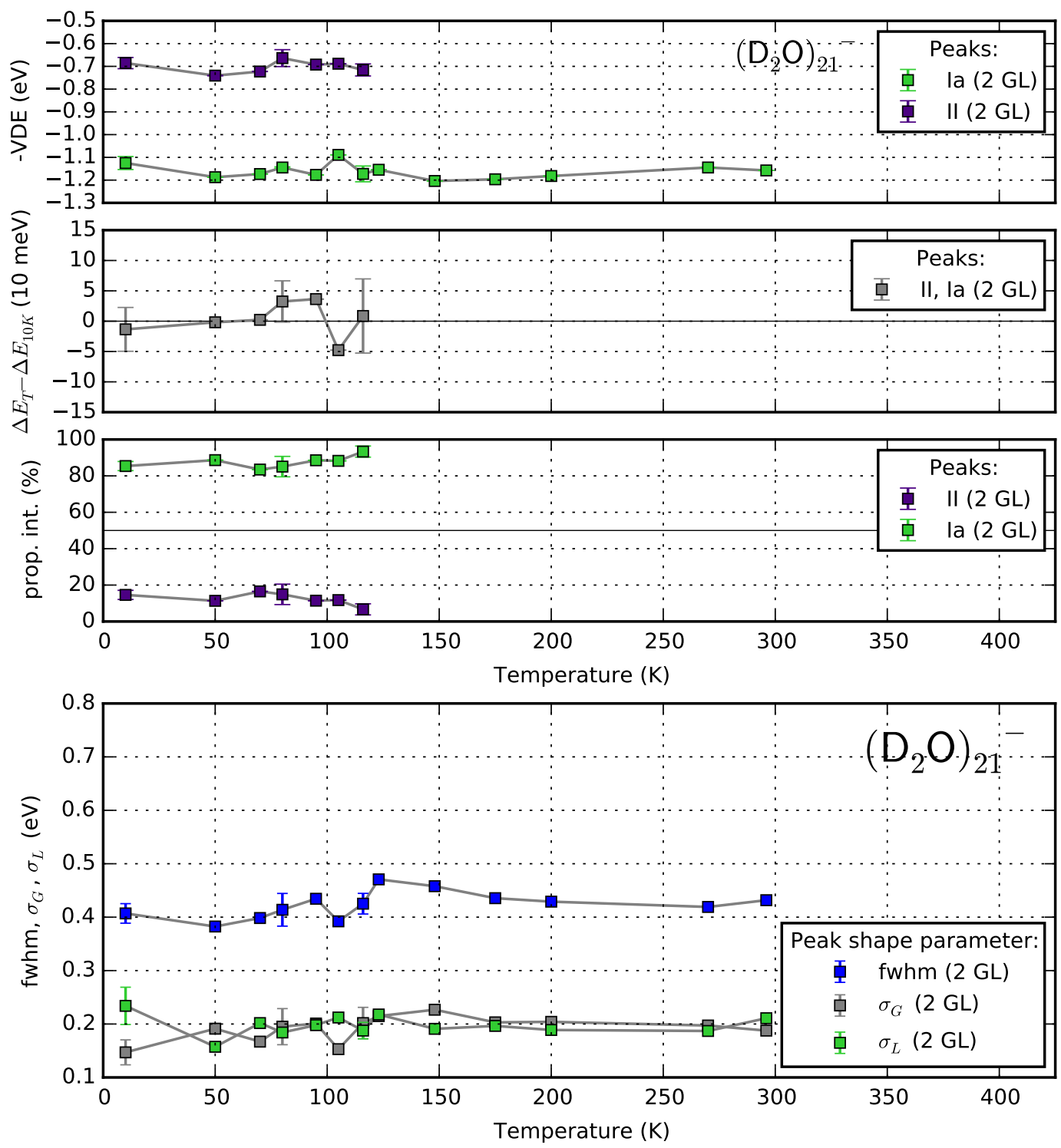

Figure B.17.: Temperature dependent evolution of the VDEs (upper three plots) and peak parameters (lower plot) of $\left(\mathrm{D}_{2} \mathrm{O}\right)_{21}{ }^{-}$. 

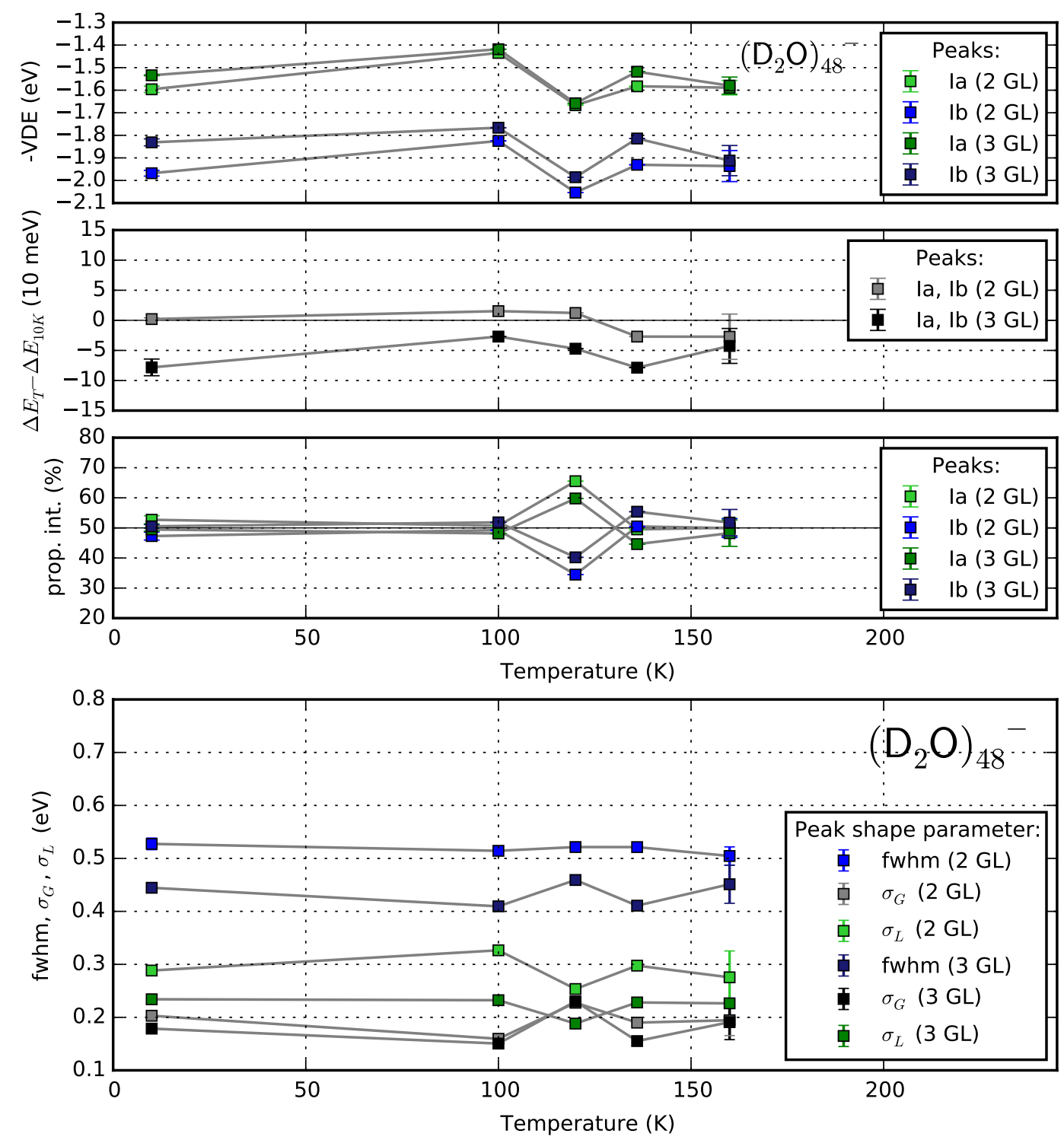

Figure B.18.: Temperature dependent evolution of the VDEs (upper three plots) and peak parameters (lower plot) of $\left(\mathrm{D}_{2} \mathrm{O}\right)_{48}{ }^{-}$. 


\section{CLUSIS a Python Framework for a CLUster Spectra Information System}

The analysis of experimental cluster spectra requires many evaluation steps. Each spectrum must first be calibrated and then transformed from flight times into more meaningful values like masses or energies. Often the spectrum is further processed in order to extract certain data, e.g. the VDEs by fitting it with GL functions in the case of the water clusters. The resulting data is then pooled to plot the size dependency of the property, which sometimes is fitted again to find a analytic form of its progression or to compare it with theoretical models.

Each evaluation step depends to a certain degree on the preceding steps. So, if one chooses e.g. to utilize a different reference spectrum for calibration, all evaluations starting at the flight time transformation has to be repeated. The former evaluation system made it very difficult to search for, reuse and share data, which often resulted in a considerable lag between data recording and evaluation.

The motivation to develop this framework was the necessity to process a large number of experimental spectra more efficiently, by making the results of the data evaluation easy to find, reusable, transparent and traceable. The framework is written in the Python programming language to ease extending it for future scientific objectives and data evaluations. The code is published on GitHub under the GPLv3 license [56]. The following gives an overview of the basic principles and the current set of features.

\section{Principles}

Each experimental spectrum consists of the actual measurement data, e.g. detector voltages for a certain time interval, plus additional data (meta data), which is essential for a complete description of the spectrum. For a cluster spectrum this would include the units of the recorded data, the cluster composition, size and ion type, the recording date and time, the type of spectrum and experimental parameter to transform the flight time spectrum, like trigger offsets, flight lengths or the laser wavelength used for photoemission. Further analysis may add more (meta) data, like fit parameters of several fit variants, scaling factors, etc. 
The measurement data and meta data of each recorded spectrum are therefore bundled into a single data set to combine all necessary informations to calculate the different representations of the spectrum and to retrace its analysis. A database containing the defining attributes can be used to search for spectra and data sets. A tagging system allows to mark subsets of spectra for separate evaluation.

\section{Features}

At the time of writing the following (incomplete) set of features are implemented:

- import of raw measurement data for PES, MS and generic spectra and merging it with essential meta data to form a "canonical" data set of the spectrum

- database search

- calculation of the respective time-of-flight, kinetic and binding energy, and mass spectra

- determination of PES calibration parameter by fitting $\mathrm{Pt}^{-}$spectra

- calibration of MS and PES

- subtracting one spectra from another, e.g. background

- scaling of spectra, e.g. comparison of alkali PES

- single and multiple GL fits of water PES

- all sorts of data visualization

- export of plots

- batch operation on lists of data sets

\section{Future Plans}

In order to make full use of the framework, a few items are still missing. First of all a better (graphical) user interface must be implemented. Collaboration and data exchange could be improved by storing the data sets in a git $1{ }^{1}$ processable form. This would, at the same time, provide for a full evaluation history.

\footnotetext{
${ }^{1}$ git is a distributed version control system used mainly for software development.
} 


\section{Bibliography}

[1] O. Andersson. Dielectric relaxation of low-density amorphous ice under pressure. Phys. Rev. Lett., 98:057602, Feb 2007. doi: 10.1103/PhysRevLett. 98.057602. URL http://link.aps.org/doi/10.1103/PhysRevLett.98. 057602 .

[2] M. Armbruster, H. Haberland, and H.-G. Schindler. Negatively charged water clusters, or the first observation of free hydrated electrons. Phys. Rev. Lett., 47:323-326, Aug 1981. doi: 10.1103/PhysRevLett.47.323. URL http://link. aps.org/doi/10.1103/PhysRevLett.47.323.

[3] P. Ayotte and M. A. Johnson. Electronic absorption spectra of size-selected hydrated electron clusters: (h[sub 2]o) [sub n] [sup -], n=6-50. J. Chem. Phys., 106(2):811-814, 1997. doi: 10.1063/1.473167. URL http://link.aip.org/ link/? JCP/106/811/1.

[4] D. Bahat, O. Cheshnovsky, U. Even, N. Lavie, and Y. Magen. Generation and detection of intense cluster beams. J. Phys. Chem., 91(10):2460-2462, 1987. URL http://pubs3.acs.org/acs/journals/doilookup?in_doi=10. 1021/j100294a003.

[5] R. Barnett, U. Landman, C. Cleveland, and J. Jortner. Size dependence of the energetics of electron attachment to large water clusters. Chem. Phys. Lett., 145(5):382-386, 4 1988. doi: 10.1016/0009-2614(88)80195-7.

[6] R. N. Barnett, R. Giniger, O. Cheshnovsky, and U. Landman. Dielectron attachment and hydrogen evolution reaction in water clusters. J. Phys. Chem. A, 115(25):7378-7391, 2011. doi: 10.1021/jp201560n. URL http://pubs . acs.org/doi/abs/10.1021/jp201560n.

[7] L. Bergmann and C. Schaefer. Lehrbuch der Experimentalphysik: Gase, Nanosysteme und Flüssigkeiten, volume 5. Gruyter, 2. edition, 2005.

[8] R. C. Bilodeau, M. Scheer, H. K. Haugen, and R. L. Brooks. Near-threshold laser spectroscopy of iridium and platinum negative ions: Electron affinities and the threshold law. Phys. Rev. A, 61:012505, Dec 1999. doi: 10.1103/ PhysRevA.61.012505. URL http://link.aps.org/doi/10.1103/PhysRevA. 61.012505 . 
[9] U. Boesl, R. Weinkauf, and E. Schlag. Reflectron time-of-flight mass spectrometry and laser excitation for the analysis of neutrals, ionized molecules and secondary fragments. Int. J. Mass Spectrom. Ion Processes, 112(2-3):121-166, Jan. 1992. ISSN 0168-1176. doi: 10.1016/ 0168-1176(92)80001-H. URL http://www.sciencedirect.com/science/ article/B6TG6-44WD083-4W/2/7980385f1bbd4e0a45f0c317d64fce4c.

[10] N. E. Bradbury and R. A. Nielsen. Absolute values of the electron mobility in hydrogen. Phys. Rev., 49:388-393, Mar 1936. doi: 10.1103/PhysRev.49.388. URL http://link .aps .org/doi/10.1103/PhysRev .49.388.

[11] A. E. Bragg, J. R. R. Verlet, A. Kammrath, O. Cheshnovsky, and D. M. Neumark. Electronic relaxation dynamics of water cluster anions. J. Am. Chem. Soc., 127(43):15283-15295, 2005. doi: 10.1021/ja052811e. URL http: //pubs.acs.org/doi/abs/10.1021/ja052811e.

[12] E. V. Browell and R. C. Anderson. Ultraviolet optical constants of water and ammonia ices. J. Opt. Soc. Am., 65(8):919-926, Aug 1975. doi: 10.1364/JOSA.65.000919. URL http://www . osapublishing . org/abstract . cfm?URI=josa-65-8-919.

[13] F. Buchner, A. Lübcke, N. Heine, and T. Schultz. Time-resolved photoelectron spectroscopy of liquids. Rev. Sci. Instrum., 81(11):113107, 2010. doi: http:// dx.doi.org/10.1063/1.3499240. URL http://scitation.aip.org/content/ aip/journal/rsi/81/11/10.1063/1.3499240.

[14] Channeltron Electron Multiplier Handbook For Mass Spectrometry Applications. Burle Industries, Inc, 2011. URL www.photonis.com/attachment. php?id_attachment=148. Publication date unknown, downloaded 2011.

[15] O. Cheshnovsky, S. H. Yang, C. L. Pettiette, M. J. Craycraft, and R. E. Smalley. Magnetic time-of-flight photoelectron spectrometer for mass-selected negative cluster ions. Rev. Sci. Instrum., 58(11):2131-2137, 1987. doi: http:// dx.doi.org/10.1063/1.1139475. URL http://scitation.aip.org/content/ aip/journal/rsi/58/11/10.1063/1.1139475.

[16] J. V. Coe, G. H. Lee, J. G. Eaton, S. T. Arnold, H. W. Sarkas, K. H. Bowen, C. Ludewigt, H. Haberland, and D. R. Worsnop. Photoelectron spectroscopy of hydrated electron cluster anions, $\left(\mathrm{H}_{2} \mathrm{O}\right)_{n=2-69}^{-}$. J. Chem. Phys., 92(6):39803982, 1990. doi: 10.1063/1.457805. URL http://link.aip.org/link/?JCP/ $92 / 3980 / 1$.

[17] J. V. Coe, S. T. Arnold, J. G. Eaton, G. H. Lee, and K. H. Bowen. Photoelectron spectra of hydrated electron clusters: Fitting line shapes and grouping 
isomers. J. Chem. Phys., 125(1):014315, 2006. doi: http://dx.doi.org/10. 1063/1.2212415. URL http://scitation.aip.org/content/aip/journal/ jcp/125/1/10.1063/1.2212415.

[18] J. V. Coe, S. M. Williams, and K. H. Bowen. Photoelectron spectra of hydrated electron clusters vs. cluster size: connecting to bulk. Int. Rev. Phys. Chem., 27(1):27-51, 2008. doi: 10.1080/01442350701783543. URL http://www.tandfonline.com/doi/abs/10.1080/01442350701783543

[19] P. G. Debenedetti and H. E. Stanley. Supercooled and glassy water. Physics Today, 56(6):40-46, 6 2003. doi: 10.1063/1.1595053. URL http://dx.doi. org/10.1063/1.1595053.

[20] W. Demtröder. Experimentalphysik 2: Elektrizität und Optik, volume 2 of Experimentalphysik. Springer Berlin Heidelberg, 5., revised edition, 2009. doi: 10.1007/978-3-642-29944-5.

[21] W. Demtröder. Experimentalphysik 3: Atome, Moleküle und Festkörper, volume 3 of Experimentalphysik. Springer Berlin Heidelberg, 4., revised edition, 2009. doi: 10.1007/978-3-642-03911-9.

[22] D. P. Fernández, A. R. H. Goodwin, and J. M. H. L. Sengers. Measurements of the relative permittivity of liquid water at frequencies in the range of 0.1 to $10 \mathrm{khz}$ and at temperatures between 273.1 and $373.2 \mathrm{k}$ at ambient pressure. Int. J. Thermophys., 16(4):929-955, 1995. ISSN 1572-9567. doi: 10.1007/ BF02093474. URL http://dx.doi .org/10.1007/BF02093474.

[23] D. Gerlich. Inhomogeneous rf fields: A versatile tool for the study of processes with slow ions. In I. P. S. A. R. Cheuk-Yiu Ng, Michael Baer, editor, StateSelected and State-to-State Ion-Molecule Reaction Dynamics. Part 1: Experiment, volume LXXXII of Advances in Chemical Physics, pages 1-176. John Wiley \& Sons, 1992. URL http://dx.doi.org/10.1002/9780470141397. ch1.

[24] D. Gerlich. Ion-neutral collisions in a 22-pole trap at very low energies. Phys. Scr., T59:256-263, 1995. URL http://stacks.iop.org/1402-4896/ $\mathrm{T} 59 / 256$.

[25] R. Gonfiantini. Standards for stable isotope measurements in natural compounds. Nature, 271(5645):534-536, Feb. 1978. URL http://dx.doi.org/ $10.1038 / 271534 \mathrm{a} 0$

[26] T. L. Guasco, B. M. Elliott, M. A. Johnson, J. Ding, and K. D. Jordan. Isolating the spectral signatures of individual sites in water networks using vibrational double-resonance spectroscopy of cluster isotopomers. J. Phys. 
Chem. Lett., 1(15):2396-2401, 2010. doi: 10.1021/jz100730q. URL http: //dx.doi.org/10.1021/jz100730q.

[27] N. I. Hammer, J.-W. Shin, J. M. Headrick, E. G. Diken, J. R. Roscioli, G. H. Weddle, and M. A. Johnson. How do small water clusters bind an excess electron? Science, 306(5696):675-679, 2004. ISSN 0036-8075. doi: 10.1126/science.1102792. URL http://science.sciencemag.org/content/ $306 / 5696 / 675$.

[28] N. I. Hammer, J. R. Roscioli, J. C. Bopp, J. M. Headrick, and M. A. Johnson. Vibrational predissociation spectroscopy of the $\left(\mathrm{H}_{2} \mathrm{O}\right)_{\mathrm{n}=6-21}{ }^{-}$clusters in the $\mathrm{OH}$ stretching region: Evolution of the excess electron-binding signature into the intermediate cluster size regime. J. Chem. Phys., 123(24):244311, 2005. doi: http://dx.doi.org/10.1063/1.2134701. URL http://scitation. aip.org/content/aip/journal/jcp/123/24/10.1063/1.2134701.

[29] N. I. Hammer, J. R. Roscioli, and M. A. Johnson. Identification of two distinct electron binding motifs in the anionic water clusters: A vibrational spectroscopic study of the $\left(\mathrm{H}_{2} \mathrm{O}\right)_{6}{ }^{-}$isomers. J. Phys. Chem. A, 109(35):78967901, 2005. doi: 10.1021/jp052144b. URL http://dx.doi.org/10.1021/ jp052144b. PMID: 16834171.

[30] E. J. Hart and J. W. Boag. Absorption spectrum of the hydrated electron in water and in aqueous solutions. J. Am. Chem. Soc., 84(21):4090-4095, 1962. doi: 10.1021/ja00880a025. URL http://pubs.acs.org/doi/abs/10.1021/ ja00880a025.

[31] M. Haruta. Size- and support-dependency in the catalysis of gold. Catal. Today, 36(1):153-166, Apr. 1997. doi: 10.1016/S0920-5861(96)00208-8. URL http://dx.doi.org/10.1016/S0920-5861(96)00208-8.

[32] J. M. Herbert and L. D. Jacobson. Structure of the aqueous electron: Assessment of one-electron pseudopotential models in comparison to experimental data and time-dependent density functional theory. J. Phys. Chem. A, 115 (50):14470-14483, 2011. doi: 10.1021/jp206391d. URL http://pubs.acs . org/doi/abs/10.1021/jp206391d.

[33] C. Hock, M. Schmidt, R. Kuhnen, C. Bartels, L. Ma, H. Haberland, and B. v.Issendorff. Calorimetric observation of the melting of free water nanoparticles at cryogenic temperatures. Phys. Rev. Lett., 103:073401, Aug 2009. doi: 10.1103/PhysRevLett.103.073401. URL http://link.aps .org/doi/10. 1103/PhysRevLett.103.073401. 
[34] C. A. Hock. Nanocalorimetry and angle-resolved photoelectron spectroscopy on sodium, aluminum, and water clusters. PhD thesis, Uni Freiburg, 2010. URL http://www.freidok.uni-freiburg.de/volltexte/7485/.

[35] T. Horio, H. Shen, S. Adachi, and T. Suzuki. Photoelectron spectra of solvated electrons in bulk water, methanol, and ethanol. Chem. Phys. Lett., 535(0): 12-16, May 2012. ISSN 0009-2614. URL http://www.sciencedirect.com/ science/article/pii/S0009261412003624

[36] L. D. Jacobson and J. M. Herbert. Theoretical characterization of four distinct isomer types in hydrated-electron clusters, and proposed assignments for photoelectron spectra of water cluster anions. J. Am. Chem. Soc., 133(49): 19889-19899, 2011. doi: 10.1021/ja208024p. URL http://pubs.acs.org/ doi/abs/10.1021/ja208024p.

[37] G. P. Johari and E. Whalley. The dielectric properties of ice $I_{h}$ in the range 272-133 K. J. Chem. Phys., 75(3):1333-1340, 1981. doi: http: //dx.doi.org/10.1063/1.442139. URL http://scitation.aip.org/content/ aip/journal/jcp/75/3/10.1063/1.442139.

[38] F.-Y. Jou and G. R. Freeman. Band resolution of optical spectra of solvated electrons in water, alcohols, and tetrahydrofuran. Can. J. Chem., 57(5): 591-597, 1979. doi: 10.1139/v79-095. URL http://dx.doi.org/10.1139/ v79-095.

[39] F.-Y. Jou and G. R. Freeman. Temperature and isotope effects on the shape of the optical absorption spectrum of solvated electrons in water. J. Phys. Chem., 83(18):2383-2387, 1979. doi: 10.1021/j100481a016. URL http://dx. doi.org/10.1021/j100481a016.

[40] A. Kammrath, J. R. R. Verlet, G. B. Griffin, and D. M. Neumark. Photoelectron imaging of large anionic methanol clusters: $(\mathrm{MeOH})_{n}^{-}(n \sim 70-460)$. J. Chem. Phys., 125(17):171102, 2006. doi: 10.1063/1.2355484. URL http: //link.aip.org/link/?JCP/125/171102/1.

[41] A. Kammrath, J. R. R. Verlet, G. B. Griffin, and D. M. Neumark. Photoelectron spectroscopy of large (water) $)_{n}^{-}(n=50-200)$ clusters at $4.7 \mathrm{eV}$. J. Chem. Phys., 125(7):076101, 2006. doi: http://dx.doi.org/10.1063/ 1.2217745. URL http://scitation.aip.org/content/aip/journal/jcp/ 125/7/10.1063/1.2217745.

[42] S. Kazachenko and A. J. Thakkar. Water nanodroplets: Predictions of five model potentials. J. Chem. Phys., 138(19):194302, 2013. doi: 10.1063/1. 4804399. URL http://link. aip.org/link/? JCP/138/194302/1. 
[43] J. Kim, I. Becker, O. Cheshnovsky, and M. A. Johnson. Photoelectron spectroscopy of the "missing" hydrated electron clusters $\left(\mathrm{H}_{2} \mathrm{O}\right)_{\mathrm{n}}{ }^{-}, n=3,5,8$ and 9: Isomers and continuity with the dominant clusters $n=6,7$ and $\geq 11$. Chem. Phys. Lett., 297(1-2):90-96, Nov. 1998. ISSN 0009-2614. URL http: //www.sciencedirect.com/science/article/pii/S0009261498011099.

[44] C. Kittel. Introduction to Solid State Physics. John Wiley \& Sons, 2004.

[45] M. Knapp, O. Echt, D. Kreisle, and E. Recknagel. Electron attachment to water clusters under collision-free conditions. J. Phys. Chem., 91(10):26012607, 1987. doi: 10.1021/j100294a031. URL http://dx.doi.org/10.1021/ j100294a031.

[46] O. Kostko. Photoelectron spectroscopy of mass-selected sodium, coinage metal and divalent metal cluster anions. PhD thesis, Fakultät für Mathematik und Physik, Albert-Ludwig-Universität Freiburg im Breisgau, 2007. URL http: //www.freidok. uni-freiburg.de/volltexte/2964/.

[47] A. Kramida, Yu. Ralchenko, J. Reader, and and NIST ASD Team. NIST Atomic Spectra Database (ver. 5.1), [Online]. Available: http:// physics.nist.gov/asd [2014, March 4]. National Institute of Standards and Technology, Gaithersburg, MD., 2013. URL http://physics.nist.gov/ PhysRefData/ASD/levels_form.html.

[48] A. Kramida, Yu. Ralchenko, J. Reader, and and NIST ASD Team. NIST Atomic Spectra Database (ver. 5.2), [Online]. Available: http://physics. nist.gov/asd [2015, April 8]. National Institute of Standards and Technology, Gaithersburg, MD., 2014. URL http://physics.nist.gov/PhysRefData/ ASD/levels_form.html.

[49] C. A. Kraus. Solutions of metals in non-metallic solvents; iv.1 material effects accompanying the passage of an electrical current through solutions of metals in liquid ammonia. migration experiments. J. Am. Chem. Soc., 30(9):13231344, 1908. doi: 10.1021/ja01951a001. URL http://dx.doi.org/10.1021/ ja01951a001.

[50] P. Kruit and F. H. Read. Magnetic field paralleliser for $2 \pi$ electronspectrometer and electron-image magnifier. J. Phys. E: Sci. Instrum., 16(4): 313, 1983. URL http://stacks.iop.org/0022-3735/16/i=4/a=016.

[51] R. E. Larsen, W. J. Glover, and B. J. Schwartz. Does the hydrated electron occupy a cavity? Science, 329(5987):65-69, 2010. doi: 10.1126/science.1189588. URL http://www . sciencemag . org/content/329/5987/65 . abstract. 
[52] S.-W. Lee, P. Freivogel, T. Schindler, and J. L. Beauchamp. Freeze-dried biomolecules: FT-ICR studies of the specific solvation of functional groups and clathrate formation observed by the slow evaporation of water from hydrated peptides and model compounds in the gas phase. J. Am. Chem. Soc., 120(45): 11758-11765, 1998. doi: 10.1021/ja982075x. URL http://dx.doi.org/10. 1021/ja982075x.

[53] A. Lübcke, F. Buchner, N. Heine, I. V. Hertel, and T. Schultz. Time-resolved photoelectron spectroscopy of solvated electrons in aqueous nai solution. Phys. Chem. Chem. Phys., 12:14629-14634, 2010. doi: 10.1039/C0CP00847H. URL http://dx.doi.org/10.1039/C0CP00847H.

[54] L. Ma, K. Majer, F. Chirot, and B. von Issendorff. Low temperature photoelectron spectra of water cluster anions. J. Chem. Phys., 131(14):144303, 2009. doi: 10.1063/1.3245859. URL http://link.aip.org/link/?JCP/131/144303/1.

[55] K. Majer. Photoelektronenspektroskopie an tiefkalten Gold- und Silberclustern. Diploma thesis, Uni Freiburg, 2008.

[56] K. Majer. Clusis - a python framework for a cluster spectra imformation system, 2012. URL https://github.com/kiranmajer/clusis.

[57] G. Makov and A. Nitzan. Solvation and ionization near a dielectric surface. J. Phys. Chem., 98(13):3459-3466, 1994. doi: 10.1021/j100064a030. URL http://dx.doi.org/10.1021/j100064a030.

[58] B. Mamyrin, V. Karataev, D. Shmikk, and V. Zagulin. The mass-reflectron, a new nonmagnetic time-of-flight mass spectrometer with high resolution. Sov. Phys.-JETP, 37(1):45-48, 1973. URL http://www.jetp.ac.ru/cgi-bin/e/ index/e/37/1/p45?a=list.

[59] O. Marsalek, F. Uhlig, T. Frigato, B. Schmidt, and P. Jungwirth. Dynamics of electron localization in warm versus cold water clusters. Phys. Rev. Lett., 105(4):043002-, July 2010. URL http://link.aps.org/doi/10.1103/ PhysRevLett.105.043002.

[60] A. Piechaczek. Gas phase calorimetry and imaging spectroscopy of water and coinage metal clusters. PhD thesis, Uni Freiburg, 2014. URL http://www. freidok.uni-freiburg.de/volltexte/9312/.

[61] J. R. Roscioli, N. I. Hammer, and M. A. Johnson. Infrared spectroscopy of water cluster anions, $\left(\mathrm{H}_{2} \mathrm{O}\right)_{\mathrm{n}=3-24}{ }^{-}$in the $\mathrm{HOH}$ bending region: Persistence of the double $\mathrm{H}$-bond acceptor (AA) water molecule in the excess electron binding site of the class I isomers. J. Phys. Chem. A, 110(24):7517-7520, 2006. doi: 
10.1021/jp062029g. URL http://dx.doi.org/10.1021/jp062029g. PMID: 16774191.

[62] J. R. Roscioli, N. I. Hammer, M. A. Johnson, K. Diri, and K. D. Jordan. Exploring the correlation between network structure and electron binding energy in the $\left(\mathrm{H}_{2} \mathrm{O}\right)_{7}{ }^{-}$cluster through isomer-photoselected vibrational predissociation spectroscopy and ab initio calculations: Addressing complexity beyond types I-III. J. Chem. Phys., 128(10):104314, 2008. doi: http://dx.doi.org/10. 1063/1.2827475. URL http://scitation.aip.org/content/aip/journal/ jcp/128/10/10.1063/1.2827475.

[63] M. Schmidt, R. Kusche, W. Kronmüller, B. von Issendorff, and H. Haberland. Experimental determination of the melting point and heat capacity for a free cluster of 139 sodium atoms. Phys. Rev. Lett., 79(1):99, Jul 1997. doi: 10. 1103/PhysRevLett.79.99. URL http://dx.doi.org/10.1103/PhysRevLett. 79.99.

[64] M. Schmidt, T. Hippler, J. Donges, W. Kronmüller, B. von Issendorff, H. Haberland, and P. Labastie. Caloric curve across the liquid-to-gas change for sodium clusters. Phys. Rev. Lett., 87(20):203402, Oct 2001. doi: 10.1103/ PhysRevLett.87.203402. URL http://dx.doi.org/10.1103/PhysRevLett. 87.203402 .

[65] J. Schnitker, K. Motakabbir, P. J. Rossky, and R. A. Friesner. A priori calculation of the optical absorption spectrum of the hydrated electron. Phys. Rev. Lett., 60:456-459, Feb 1988. doi: 10.1103/PhysRevLett.60.456. URL http://link.aps.org/doi/10.1103/PhysRevLett.60.456.

[66] A. T. Shreve, T. A. Yen, and D. M. Neumark. Photoelectron spectroscopy of hydrated electrons. Chem. Phys. Lett., 493(4-6):216 - 219, 2010. ISSN 00092614. doi: 10.1016/j.cplett.2010.05.059. URL http://www.sciencedirect. com/science/article/pii/S0009261410007232.

[67] A. T. Shreve, M. H. Elkins, and D. M. Neumark. Photoelectron spectroscopy of solvated electrons in alcohol and acetonitrile microjets. Chem. Sci., 4:16331639, 2013. doi: 10.1039/C3SC22063J. URL http://dx.doi.org/10.1039/ C3SC22063J.

[68] K. R. Siefermann, Y. Liu, E. Lugovoy, O. Link, M. Faubel, U. Buck, B. Winter, and B. Abel. Binding energies, lifetimes and implications of bulk and interface solvated electrons in water. Nature Chem., 2(4):274-279, Apr. 2010. ISSN 1755-4330. URL http://dx.doi.org/10.1038/nchem.580.

[69] A. K. Soper and C. J. Benmore. Quantum differences between heavy and light water. Phys. Rev. Lett., 101:065502, Aug 2008. doi: 10.1103/PhysRevLett. 
Bibliography

101.065502. URL http://link.aps.org/doi/10.1103/PhysRevLett.101. 065502 .

[70] C. W. Stoermer, S. Gilb, J. Friedrich, D. Schooss, and M. M. Kappes. A high resolution dual mass gate for ion separation in laser desorption/ionization time of flight mass spectrometry. Rev. Sci. Instrum., 69(4):1661-1664, 1998. doi: http://dx.doi.org/10.1063/1.1148823. URL http://scitation.aip. org/content/aip/journal/rsi/69/4/10.1063/1.1148823.

[71] Y. Tang, H. Shen, K. Sekiguchi, N. Kurahashi, T. Mizuno, Y.-I. Suzuki, and T. Suzuki. Direct measurement of vertical binding energy of a hydrated electron. Phys. Chem. Chem. Phys., 12:3653-3655, 2010. doi: 10.1039/B925741A. URL http://dx.doi .org/10.1039/B925741A.

[72] L. Turi. Hydrated electrons in water clusters: Inside or outside, cavity or noncavity? J. Chem. Theory Comput., 11(4):1745-1755, 2015. doi: 10.1021/ ct501160k. URL http://dx.doi.org/10.1021/ct501160k.

[73] L. Turi. On the applicability of one- and many-electron quantum chemistry models for hydrated electron clusters. J. Chem. Phys., 144(15):154311, 2016. doi: http://dx.doi.org/10.1063/1.4945780. URL http://scitation. aip.org/content/aip/journal/jcp/144/15/10.1063/1.4945780.

[74] L. Turi and P. J. Rossky. Theoretical studies of spectroscopy and dynamics of hydrated electrons. Chem. Rev., 112(11):5641-5674, 2012. doi: 10.1021/ cr300144z. URL http://pubs.acs.org/doi/abs/10.1021/cr300144z.

[75] L. Turi, W.-S. Sheu, and P. J. Rossky. Characterization of excess electrons in water-cluster anions by quantum simulations. Science, 309(5736):914-917, 2005. doi: 10.1126/science.1115808. URL http://www.sciencemag.org/ content/309/5736/914.abstract.

[76] T. R. Tuttle and S. Golden. Shape stability of solvated-electron optical absorption bands. part 1.-experimental basis. J. Chem. Soc., Faraday Trans. 2, 77:873-888, 1981. doi: 10.1039/F29817700873. URL http://dx.doi .org/10. 1039/F29817700873.

[77] J. R. R. Verlet, A. E. Bragg, A. Kammrath, O. Cheshnovsky, and D. M. Neumark. Observation of large water-cluster anions with surface-bound excess electrons. Science, 307(5706):93-96, 2005. doi: 10.1126/science.1106719. URL http://www.sciencemag.org/content/307/5706/93.abstract.

[78] S. G. Warren and R. E. Brandt. Optical constants of ice from the ultraviolet to the microwave: A revised compilation. J. Geophys. Res., 113(D14):D14220, 
2008. ISSN 2156-2202. doi: 10.1029/2007JD009744. URL http://dx.doi. org/10.1029/2007 JD009744. D14220.

[79] R. Weinkauf, K. Walter, C. Weickhardt, U. Boesl, and E. Schlag. Laser tandem mass spectrometry in a time of flight instrument. Z. Naturforsch., 44a:12191225, 1989.

[80] W. Weyl. Ueber Metallammonium-Verbindungen. Ann. Phys., 197(4):601612, 1864. ISSN 1521-3889. doi: 10.1002/andp.18641970407. URL http: //dx.doi.org/10.1002/andp.18641970407.

[81] W. C. Wiley and I. H. McLaren. Time-of-flight mass spectrometer with improved resolution. Rev. Sci. Instrum., 26(12):1150-1157, 1955. URL http://dx.doi.org/10.1063/1.1715212.

[82] R. M. Young, M. A. Yandell, S. B. King, and D. M. Neumark. Thermal effects on energetics and dynamics in water cluster anions $\left(\mathrm{H}_{2} \mathrm{O}\right)_{n}^{-}$. J. Chem. Phys., 136(9):094304, 2012. doi: 10.1063/1.3689439. URL http://link.aip.org/ link/?JCP/136/094304/1. 